\title{
DRIFT OF ZOOPLANKTON, BENTHOS, AND LARVAL FISH AND DISTRIBUTION OF MACROPHYTES AND LARVAL FISH IN THE ST. MARYS RIVER, MICHIGAN, DURING WINTER AND SUMMER, 1985
}

\section{David J. Jude, Michael Winnell, Marlene S. Evans, Frank J. Tesar, and Richard Futyma}

Prepared under Contract DACW 35-85-C-0005

for U.S. Army Corps of Engineers, Detroit District, Detroit, Michigan 48231

\section{Great Lakes Research Division \\ Special Report No. 124}

The University of Michigan

Ann Arbor, Michigan 48109 


\section{ACKNOWLEDGMENTS}

We are indebted to the many colleagues who assisted in the collection of data for this study under many times hazardous winter and cold spring conditions. Winter drift sampling was made tolerable by our resident ombudsman Glen Tompkins, who purchased equipment, scouted resting sites, and whose knowledge of the area facilitated sampling immensely. Others performing yeoman's duty during winter included: Curt Ritter, Heang Tin, and Jim Wojcik. Spring larval fish sampling was conducted with the able assistance of Phil schneeberger, Phil Hirt, Heang Tin, Mary Sweeney, and Pam Mansfield. Summer drift collections were made with help from Mary Sweeney and Phil Hirt. Chuck Elzinga assisted with macrophyte collections. Heang Tin processed most larval fish samples. Benthos samples were processed by Connie Achtenburg, Jim Wojcik, Roger LaDronka, Chuck Elzinga, and Heang Tin. Zooplankton samples were counted by Mohammed Omair and biomass data generated by Phil Hirt and Mary Sweeney. We gratefully acknowledge the data entry and computer work provided by Connie Achtenburg.

We thank Tom Edsall of the Great Lakes Fishery Laboratory for permission to use some of their sampling gear and Tom Poe, Jarl Hiltunen, and Chuck Hatcher for good advice on how to sample and what conditions to expect. We thank H.K. Soo of the Great Lakes Environmental Research Lab for use of their current meter during winter and Jules Gelderhoos, Dearborn Campus, University of Michigan, for use of their current meter during summer. Al Ballert of the Great Lakes Commission provided information for our report and Captain Taylor of the U.S. Coast Guard in Sault Ste. Marie provided advice and radio contact for safety purposes. The University of Michigan Biological Station provided the use of their Boston Whaler and vehicles for macrophyte sampling during summer and various pieces of equipment for our winter activities. We thank Claire Schelske for use of the photometer for our summer measurements.

Mary Sweeney, Heang Tin, and Scott DeBoe provided figures for the report, and Bev McClellan entered corrections, new text, and some tables. We gratefully acknowledge the contribution that Pam Mansfield made in putting together and producing the final report. Steve Schneider provided very useful editorial contributions. We thank Walt Duffy, Jim Diana, and Jim Bowers for critical comments. This project was funded by the U.S. Army Corps of Engineers, Detroit District. 
TABLE OF CONTENTS

ACKNOWLEDGMENTS.............................. i

LIST OF APPENDIXES .......................... v

INTRODUCTION................................ I

METHODS..................................

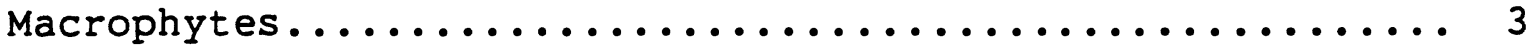

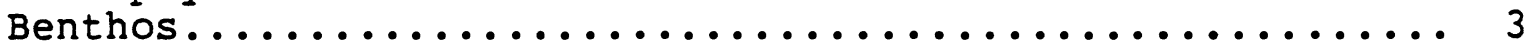

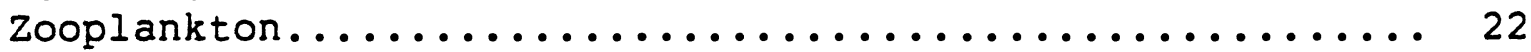

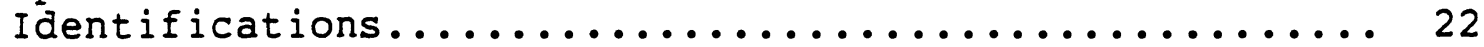

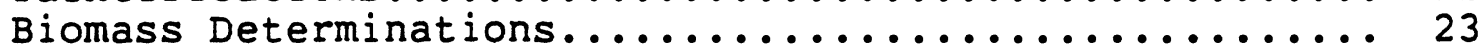

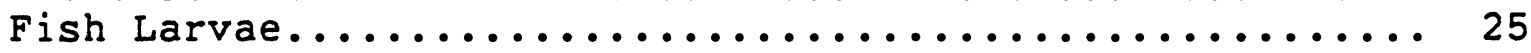

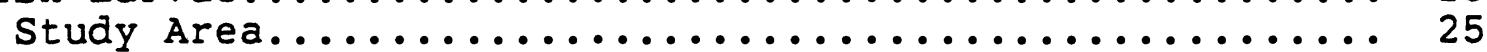

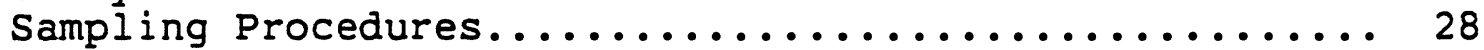

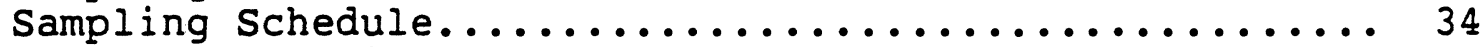

Sample Processing.......................... 34

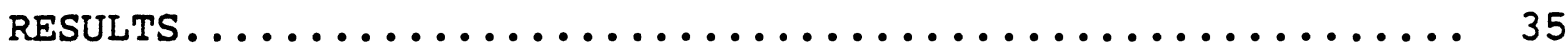

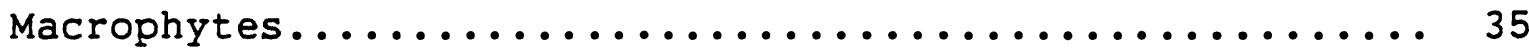

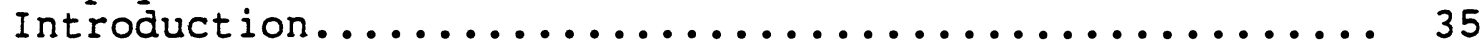

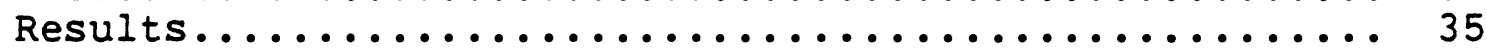

Drift: Benthos, Fish Larvae, Fish Eggs,

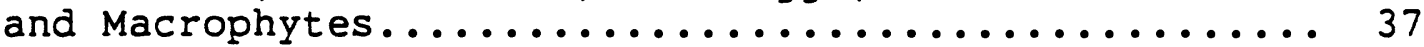

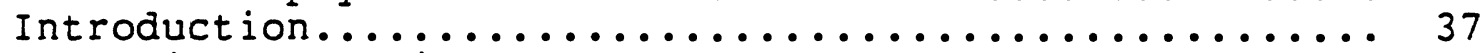

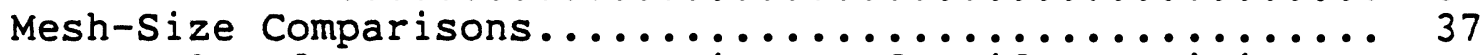

Seasonal and Transect Comparisons of Drift Densities... 51

Seasonal and Transect Comparisons of Drift Rates

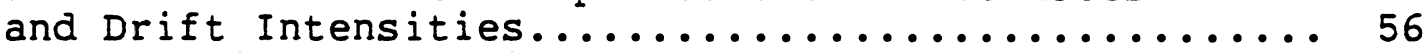

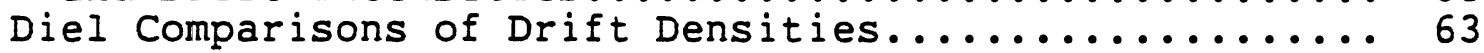

Depth Strata Comparisons of Drift Densities.......... 68

Station Density Comparisons Within Transects......... 74

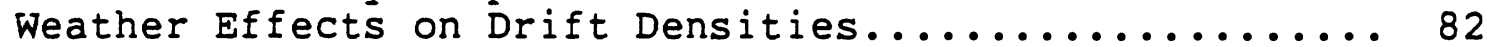

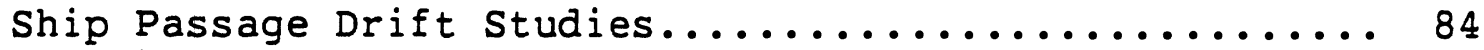

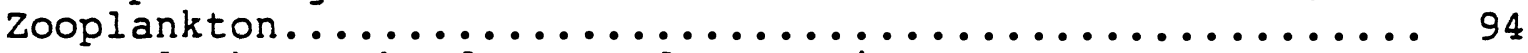

Zooplankton Abundance and Community Structure........ 94

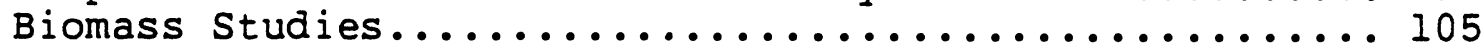

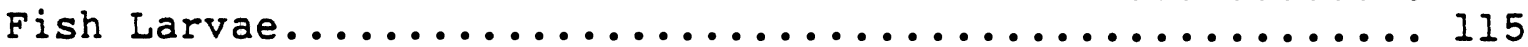

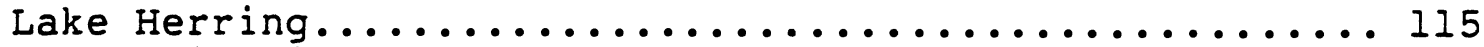

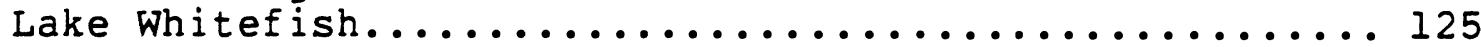

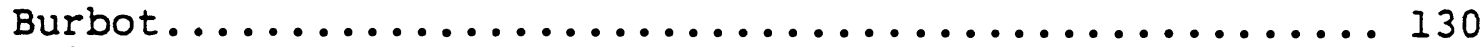

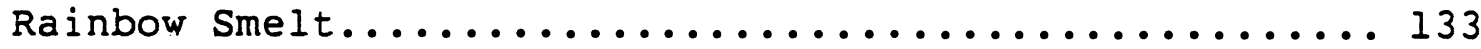

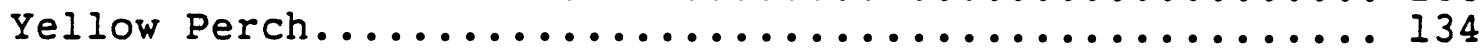

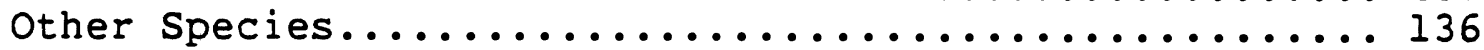

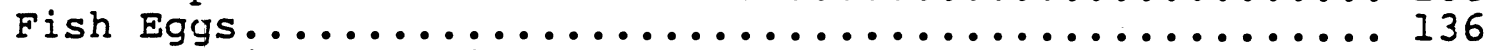

Summer Drift and Biomass of Larval Fish............ 136 


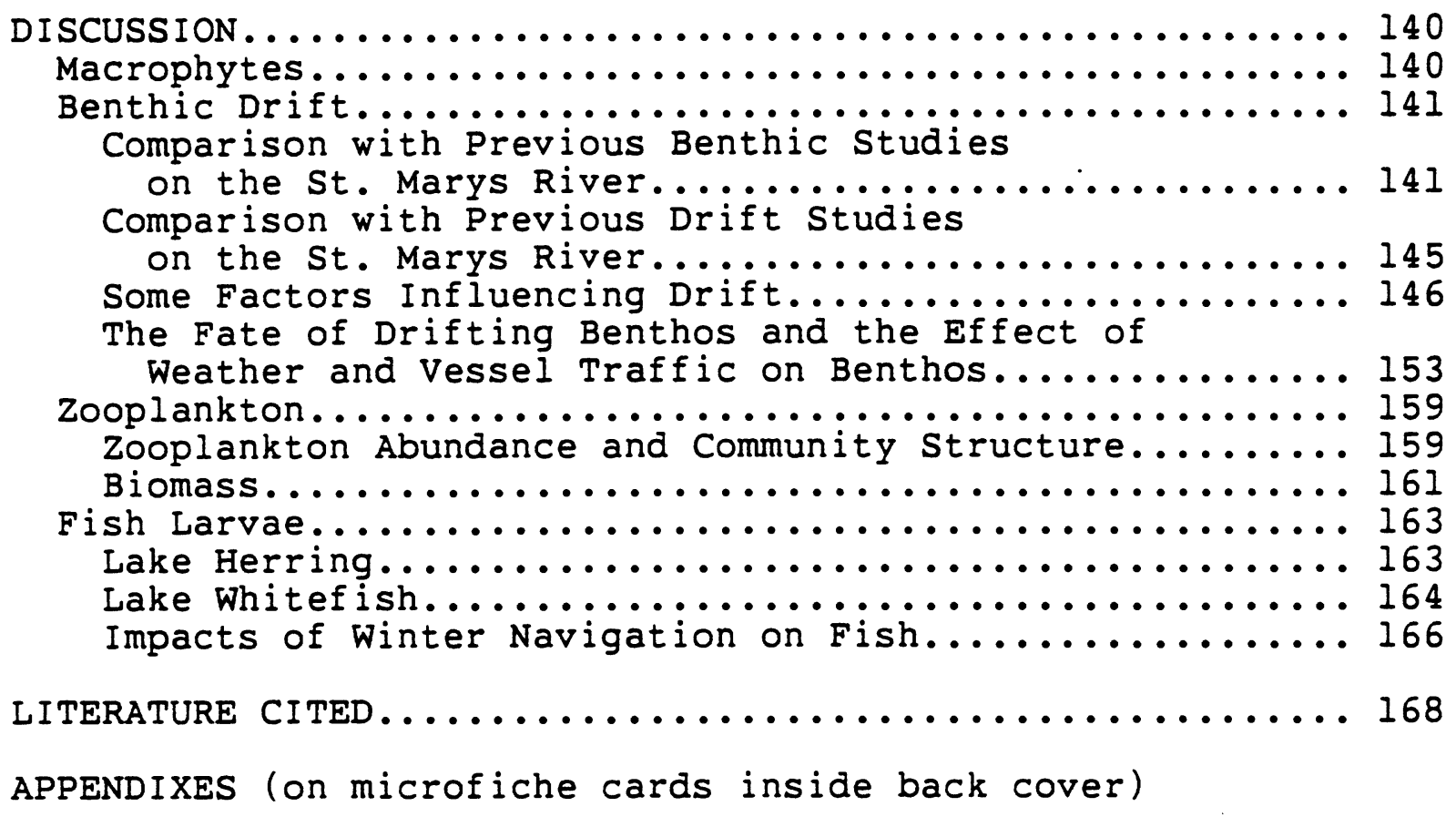


Appendixes 1-6. Length of time sampled, current velocity, and total volume of water filtered for drift samples.

Appendix 7. Winter and summer water temperature (C) for drift samples.

Appendixes 8-27. Mean densities, standard error, and percent of total benthos for Frechette Point winter drift samples.

Appendixes 28-47. Mean densities, standard error, and percent of total benthos for Frechette Point summer drift samples.

Appendixes 48-73. Mean densities, standard error, and percent of total benthos for Lake Nicolet winter drift samples.

Appendixes 74-101. Mean densities, standard error, and percent of total benthos for Lake Nicolet summer drift samples.

Appendixes 102-114. Mean densities, standard error, and percent of total benthos for Point aux Frenes winter drift samples.

Appendixes 115-134. Mean densities, standard error, and percent of total benthos for Point aux Frenes summer drift samples.

Appendixes 135-154. Macrophy te, benthos, fish larvae, and seston dry weight biomass estimates for Frechette Point winter drift samples.

Appendixes 155-174. Macrophyte, benthos, fish larvae, and seston dry weight biomass estimates for Frechette Point summer drift samples.

Appendixes 175-200. Macrophyte, benthos, fish larvae, and seston dry weight biomass estimates for Lake Nicolet winter drift samples.

Appendixes 201-228. Macrophyte, benthos, fish larvae, and seston dry weight biomass estimates for Lake Nicolet summer drift samples.

Appendixes 229-241. Macrophyte, benthos, fish larvae, and seston dry weight biomass estimates for Point aux Frenes winter drift samples. $\underline{\text { PAGE }}$

A -1 to $A-15$

A -16 to $A-20$

A -21 to $A-40$

A -41 to $A-73$

A -74 to $A-99$

$\mathrm{A}-100$ to $\mathrm{A}-128$

$\mathrm{A}-129$ to $\mathrm{A}-141$

$A-142$ to $A-161$

$A-162$ to $A-181$

$A-182$ to $A-201$

$A-202$ to $A-227$

$\mathrm{A}-228$ to $\mathrm{A}-255$

$\mathrm{A}-256$ to $\mathrm{A}-268$ 
Appendixes 242-261. Macrophyte, benthos, fish larvae, and seston dry weight biomass estimates for Point aux Frenes summer drift samples.

$\mathrm{A}-269$ to $\mathrm{A}-288$

Appendixes 262-266. Light measurements: energy flux and extinction coefficients for Izaak Walton Bay, Lake Nicolet, western Lake Munuscong, eas tern Lake Munuscong, and Raber Bay.

$\mathrm{A}-289$ to $\mathrm{A}-304$

Appendix 267. Zooplankton abundance, composition, and statistics for various dates, stations, mesh sizes, and sampling periods on the St. Marys River.

$A-305$ to $A-327$ 


\section{INTRODUCTION}

The St. Marys River, the connecting channel between Lake Superior and Lakes Huron and Michigan, is part of the vast surface waters of the Great Lakes and therefore bears a considerable amount of recreational boat and commercial ship traffic. Several questions have been raised regarding the impact of large ship passage on the indigenous fish and wildiffe. In addition, extended winter operation of the lock complex at sault Ste. Marie, Michigan has been proposed to 31 January \pm 2 wk. This contract (DACW 35-85-C-0005) was prepared by the U.S. Army Corps of Engineers, Detroit District, to obtain data to allow predictions regarding the impact of winter shipping to as late as 15 February on the St. Marys River. The results will be incorporated into a supplemental environmental impact statement concerning extension of the operating season of the lock facilities at Sault Ste. Marie to 31 January \pm 2 wk. Because of the surge and currents that ships can generate, they may have substantial effects on the spawning grounds of such important $f$ ish as lake whitefish (Coregonus clupeaformis), lake herring (c. aItedii), and other recreationaliy important species. There is also concern about the effects of ship passage on benthic invertebrate communities, and how sediment resuspension, possibly caused by ship traffic, may impact the primary producers in the system, especially the aquatic macrophytes.

The fauna of the St. Marys River is a combination of animals drifting or moving in from Lakes Superior, Huron, and Michigan and those produced within the system. The river includes backwater areas; riverine channels, embayments, and lakes; scattered wetlands; and other estuarine habitat. A recent report (Duffy et al. 1987) summarizes a considerable amount of physical, chemical, and biological information, including ours, on the St. Marys River ecosystem.

To examine some of the impacts of winter and summer navigation on the indigenous fauna and flora, we studied three components of the St. Marys River ecosystem: the macrophyte community, the drifting benthos and zooplankton community, and the larval fish community. The macrophyte studies were aimed at determining how far the natural boundary of plant growth extended into the river. We also collected a series of spatially and temporally spaced light measurements in the river to obtain some information on light extinction coefficients, thereby establishing what turbidity currently exists in the St. Marys River and how that might affect or limit plant growth.

The second thrust of the study was aimed at documenting invertebrate drift in the St. Marys River during winter ice cover and during summer. For this purpose, sampling stations were established along three transects across the river. Several 
depth strata were sampled at each of these transects. Drift samples were collected with two different mesh sizes to efficiently sample both the benthos and zooplankton. Sampling was performed during the day and night so no major periods of peak drift would be missed. All invertebrates were removed from the sample as well as plant fragments, detritus, and $f$ ish larvae. All organisms from each of these groups were then weighed and biomass determined.

Lastly, we conducted larval fish sampling in the spring to attempt to document lake herring and lake whitefish spawning grounds in the St. Marys River. Transects were located from the power canal in Sault harbor to the southern end of Neebish Island. Fish larvae were sampled at night to reduce net avoidance. At each transect, three stations were established; one was at $1 \mathrm{~m}$, one at $2 \mathrm{~m}$, and one was in the channel. One station was located near the head of the St. Marys River where Lake Superior enters, to establish whether fish larvae were drifting in from Lake Superior or Izaak Walton Bay: Results from these studies were used to draw conclusions regarding the impact ship passage might have on the respective components of the aquatic ecosystem. 


\section{METHODS}

\section{MACROPHYTES}

All sampling was done from a $5-\mathrm{m}$ Boston Whaler motor boat equipped with a Datamarine electronic depth finder. To locate the maximum depth to which submerged macrophyte beds extended at a site, samples of bottom-growing plants were taken with a grappling hook along several transects perpendicular to the depth contours. This estimate was refined by using a Ponar grabsampler to take a number of bottom samples, each covering approximately $620 \mathrm{~cm}^{2}$ of substrate, at depths within about $\pm 0.6 \mathrm{~m}$ of the original estimate. After assigning a depth to the outer boundary of macrophyte beds, the boat was moved over that depth contour for a distance of $1 \mathrm{~km}$, and Ponar grab-samples were taken at intervals of approximately $30 \mathrm{~m}$, for a minimum of 30 samples. In practice, sampling was done over a depth range within $0.5 \mathrm{~m}$ of the estimated boundary depth. Plants retrieved in each sample were placed in a labeled plastic bag and were later identified in the laboratory. Specimens were identified with the aid of manuals by Fassett (1957), Voss (1972), and Prescott (1962), and the herbarium of the University of Michigan Biological Station, Pellston, Michigan.

During the same day that bottom sampling at the outer macrophyte boundary was done, and on four other occasions, light penetration measurements were taken over plant beds in three locations in the vicinity of the $1-\mathrm{km}$ transect in each of the five portions of the river (Fig. I). A LI-COR model LI-185 quantum meter was used to measure energy flux at photosynthetically active wavelengths $(400-700 \mathrm{~nm})$. Measurements were taken at the water surface, at $1-m$ depth intervals, and at the maximum depth (which depended on site, ca. $20 \mathrm{~cm}$ above the bottom) to which the light sensor could be lowered. The data from each light-measurement location were used to calculate vertical light extinction coefficients by the least squaremethods (Lind 1979).

Five sites along the length of the river were chosen for study, one in each of the following parts of the river: Izaak Walton Bay (Mosquito Bay), Lake Nicolet, western Lake Munuscong, eastern Lake Munuscong, and the Raber Bay--Maud Bay area (see Fig. 2). Plant sampling and light measurements were done during July and August, 1985.

\section{BENTHOS}

Samples of the macrophytic, zooplanktonic, benthic, larval $f$ ish, and $f$ ish egg drift were collected during a period of ice 


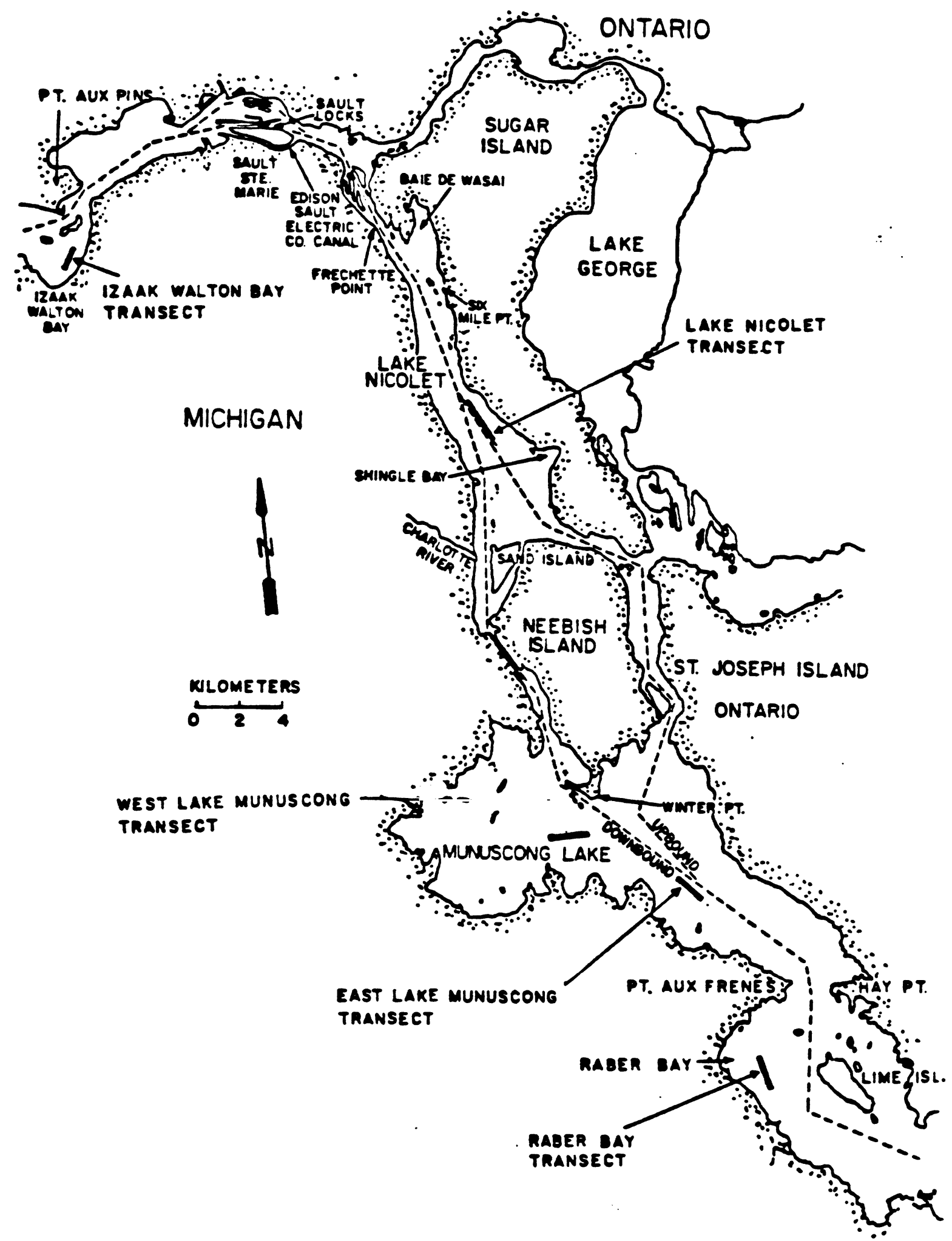

Figure 1. Location of the five transects (dark bar) where the depth boundary of submerged macrophytes and light penetration were measured in the St. Marys River, 1985. 


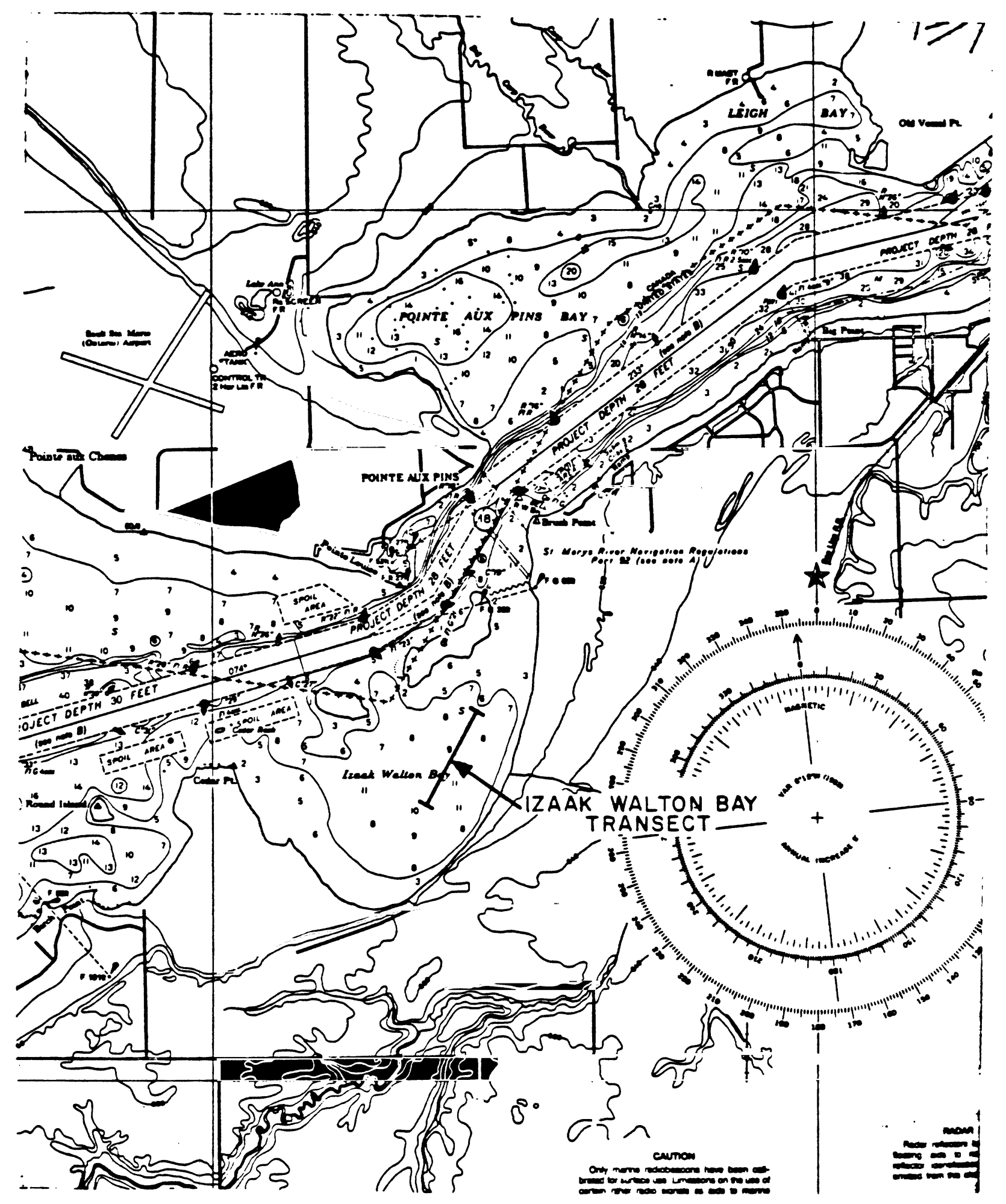

Figure 2. Detailed location maps for the five transects established to sample macrophytes on the St. Marys River, 1985. Taken from NOAA Chart 14884. 


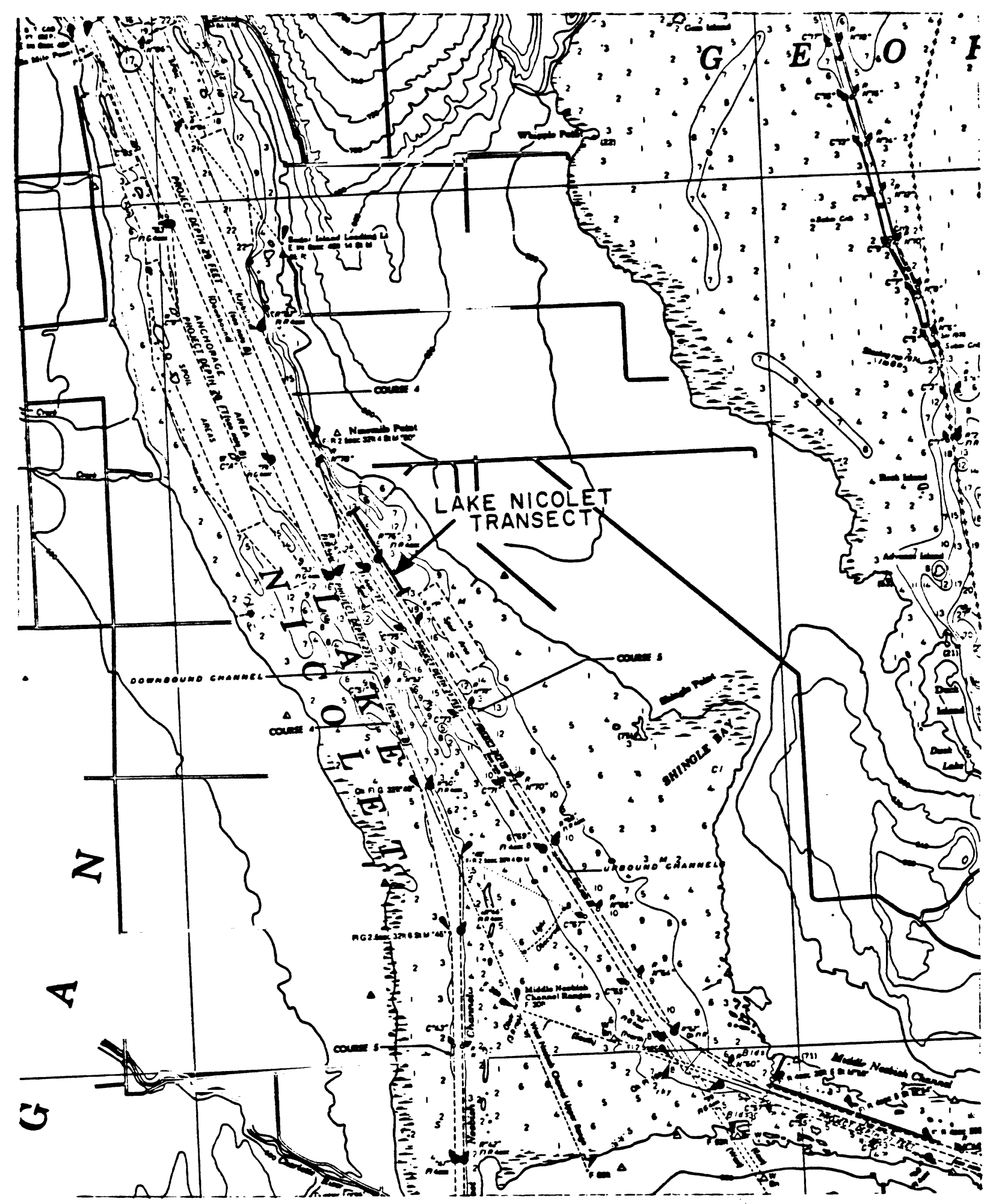

Figure 2. Continued. 


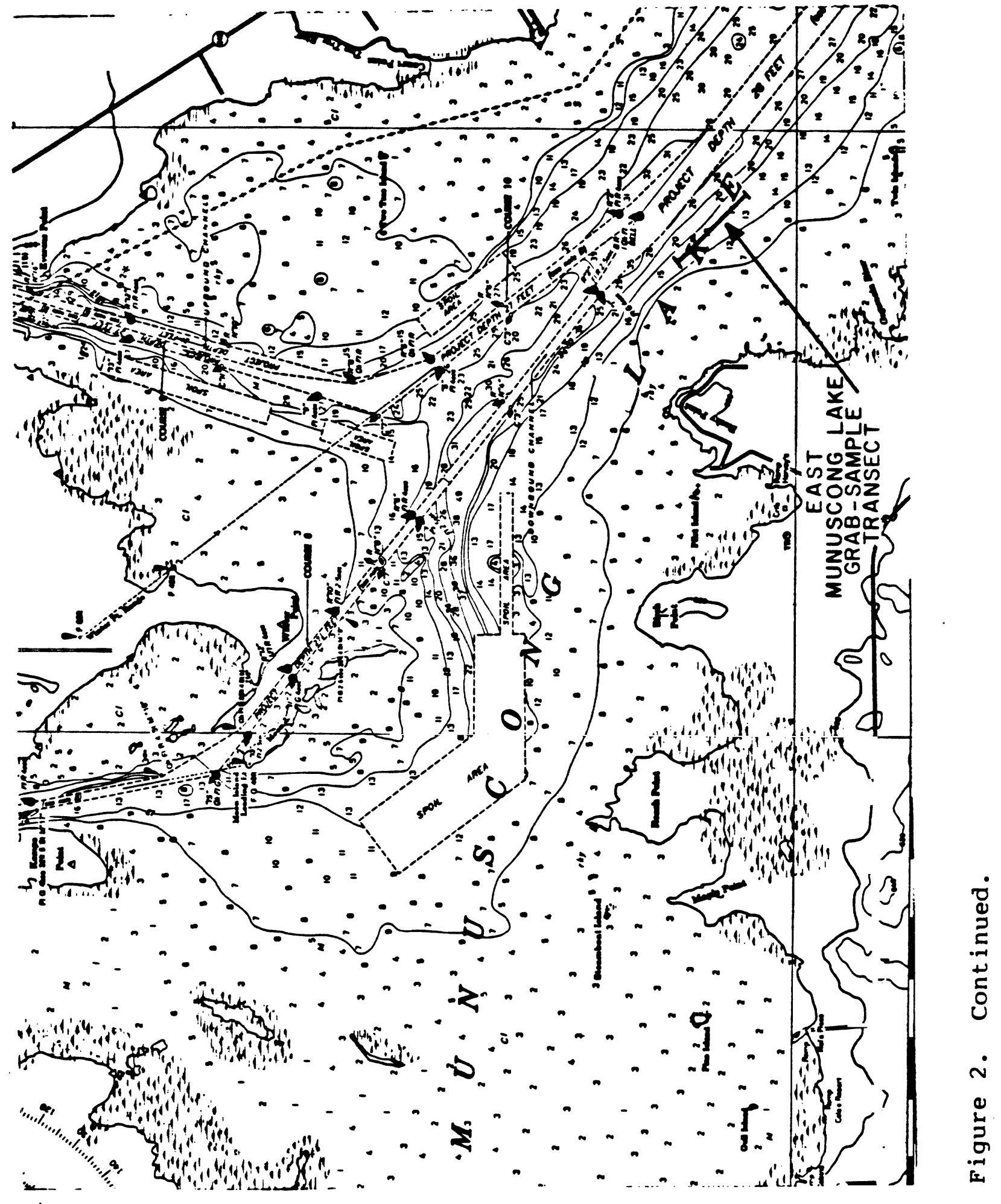




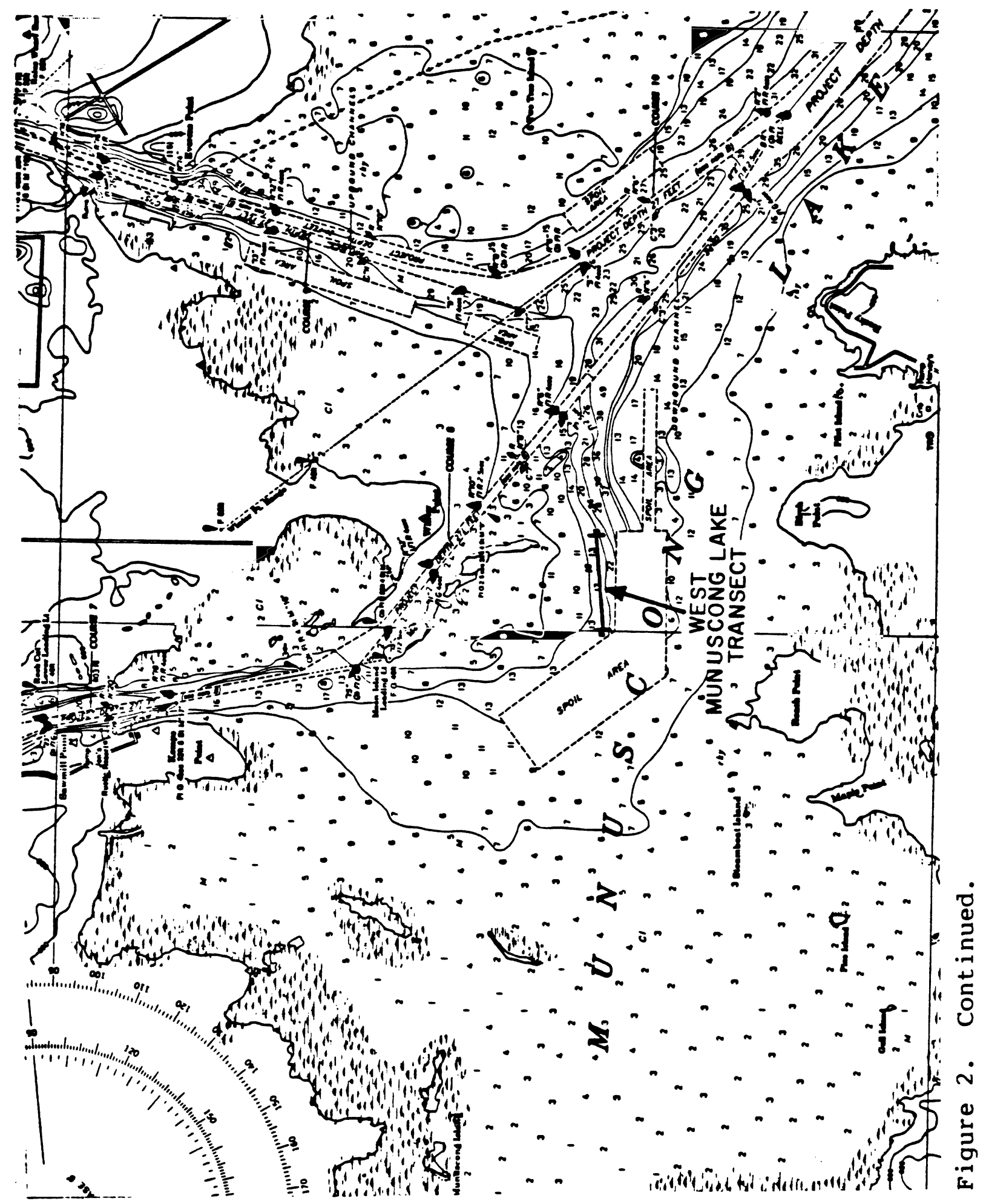




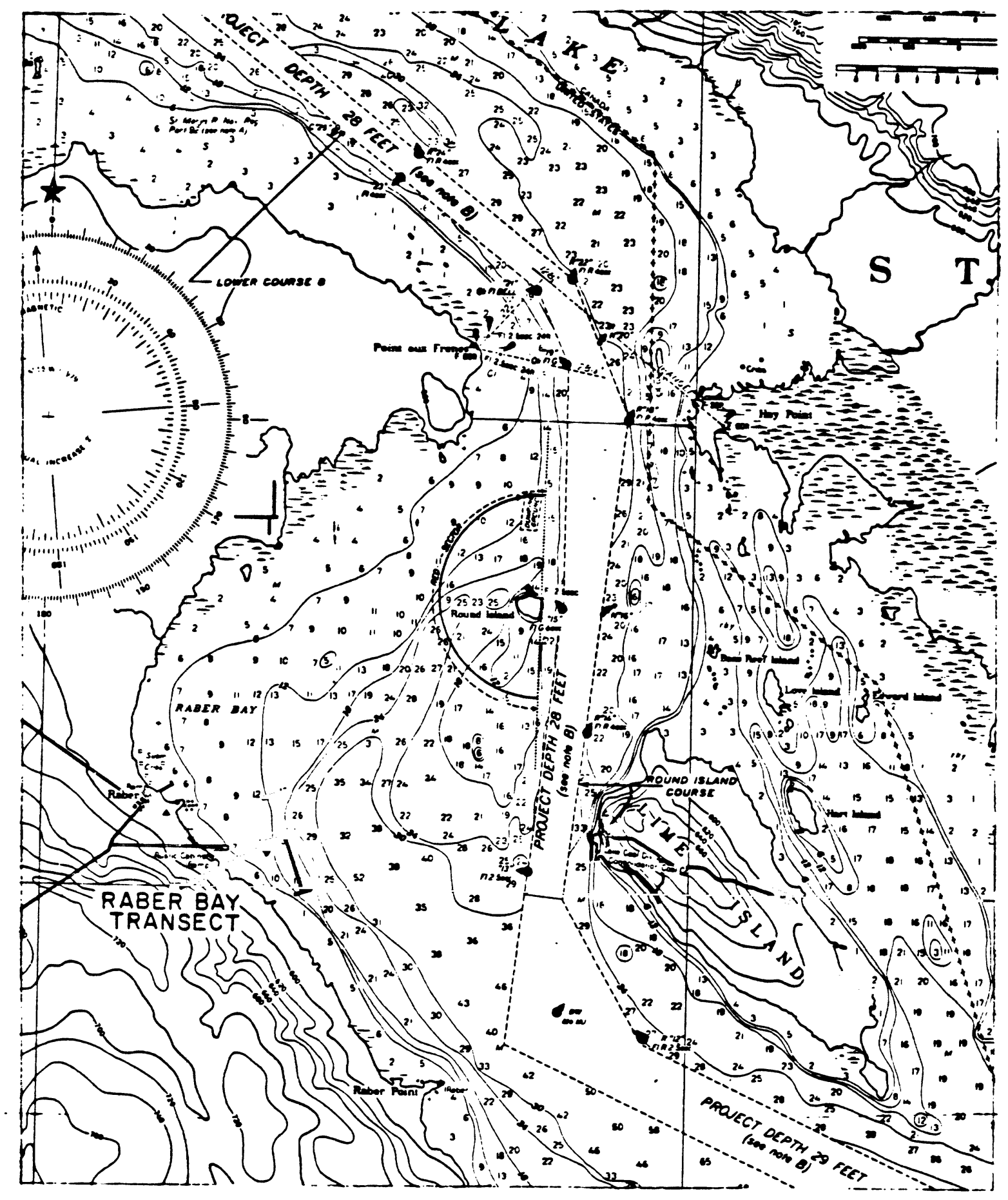

Figure 2. Concluded. 
cover (23 February - 7 March 1985) and a period free of ice (2-14 June 1985) from three transects located at Frechette Point, lower Lake Nicolet, and Point aux Frenes (Lake Munuscong) (Figs. 3,4). At the Frechette Point and Pt. aux Frenes transects, sample stations were established at 1-, 2-, and 3-m depths on either side of the navigation channel for a total of six stations at each transect. A seventh station at each site was located in the navigation channel at a water depth of 9-10 m. In lower Lake $\mathrm{Nicolet}$, the number of stations was increased to nine because the navigation channel is divided into upbound and downbound

channels. Like the two previous transects, stations were located at 1-, 2-, and 3-m depths on the shoreward side of both channels, and a station was established in each navigation channel. A final station was located at $2 \mathrm{~m}$ in the central area of the lake between the two channels (see Fig. 4). Although $988 \mathrm{drift}$ samples were scheduled to be collected, the final number of samples collected was 964 .

The vertical water column was sampled for drift at subsurface, mid-depth, and near-bottom locations, depending upon station depth. At $1-m$ stations, drift samples were collected near bottom. At 2- and 3-m stations, samples were collected at mid-depth and near bottom. Finally, at all navigation channel stations, samples were collected from all three water depth locations.

Two simultaneous replicates were collected four times at each depth stratum at each station during approximate $12-\mathrm{h}$ time periods corresponding to Day 1, Night 1, Day 2, and Night 2. Day sampling generally occurred between 1000 and $1700 \mathrm{~h}$ EST, while winter night sampling occurred between 1900 and $2300 \mathrm{~h}$ and summer night sampling between 2200 and $0200 \mathrm{~h}$.

The drift at all stations was sampled with nets that were $1 \mathrm{~m}$ in length with a 29-cm diameter mouth; mesh size was $355 \mu \mathrm{m}$. In addition, the drift at all $3-\mathrm{m}$ and navigation stations during Day $I$ and Night 1 time periods was sampled using a similarly sized drift net but of finer mesh size $(153 \mu \mathrm{m})$. At all stations, except navigation channel sites, nets of similar mesh size were attached at the top and bottom of the net ring to crossrods by slipping the crossrods through the rope which secured the net to the ring (Fig. 5). Each net was clipped to the center pole such that it was attached at three locations. Crossrods were fastened to a sleeve such that the whole apparatus, including nets, rotated about a central, 16-mm, T6160 aluminim support rod which followed the design of poe and Edsall (1982). Depending on the vertical water depth required, setscrew stops were affixed above and below the crossrod apparatus to prevent vertical movement. In winter, $0.3-$ by $1-\mathrm{m}$ rectangular holes were bored into the ice using a gas-powered auger. The sampling unit with nets attached was then lowered into the water 


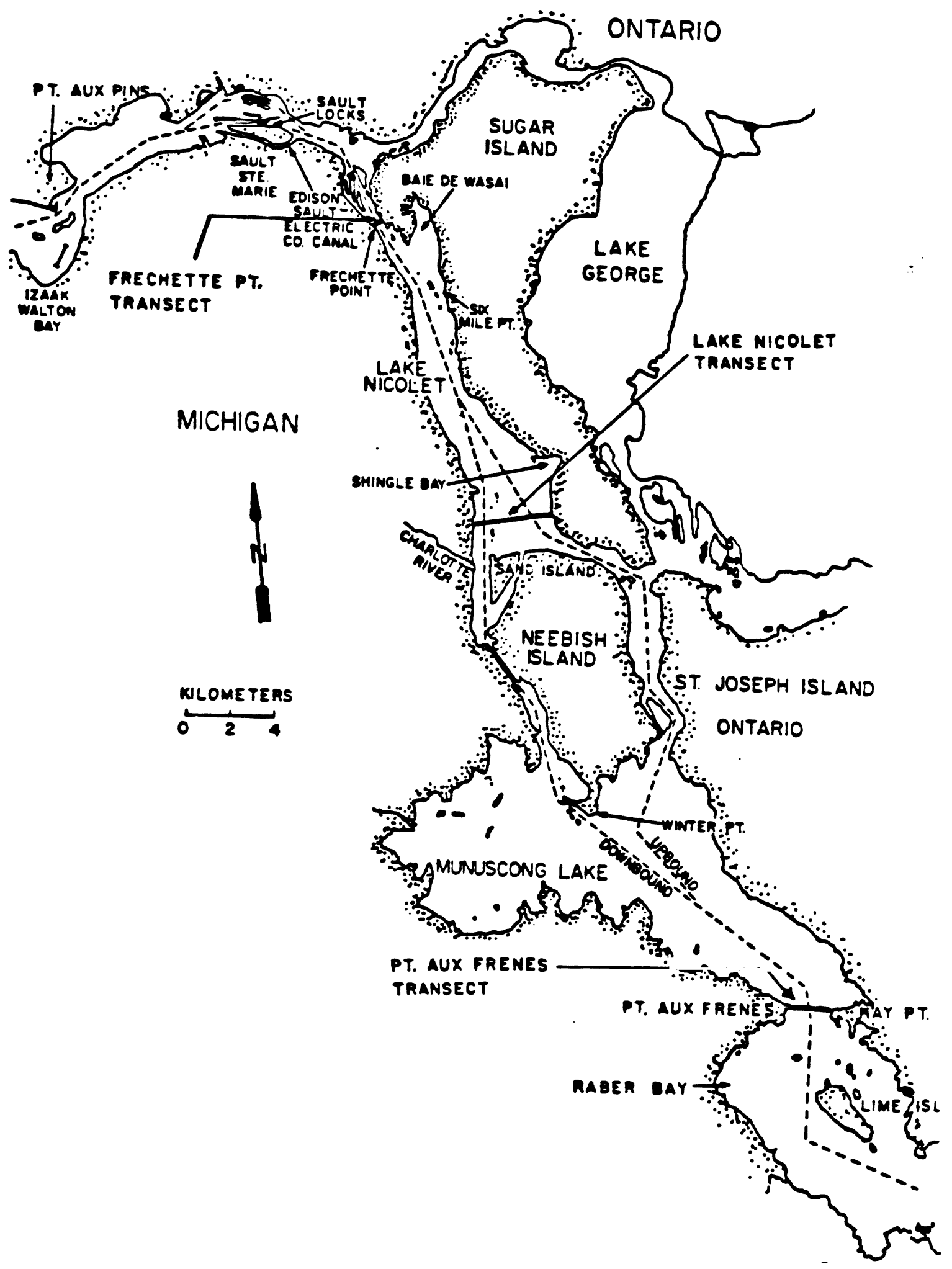

Figure 3. Location of the three transects (dark bar) where benthic drift was sampled in the St. Marys River during winter and summer, 1985. 


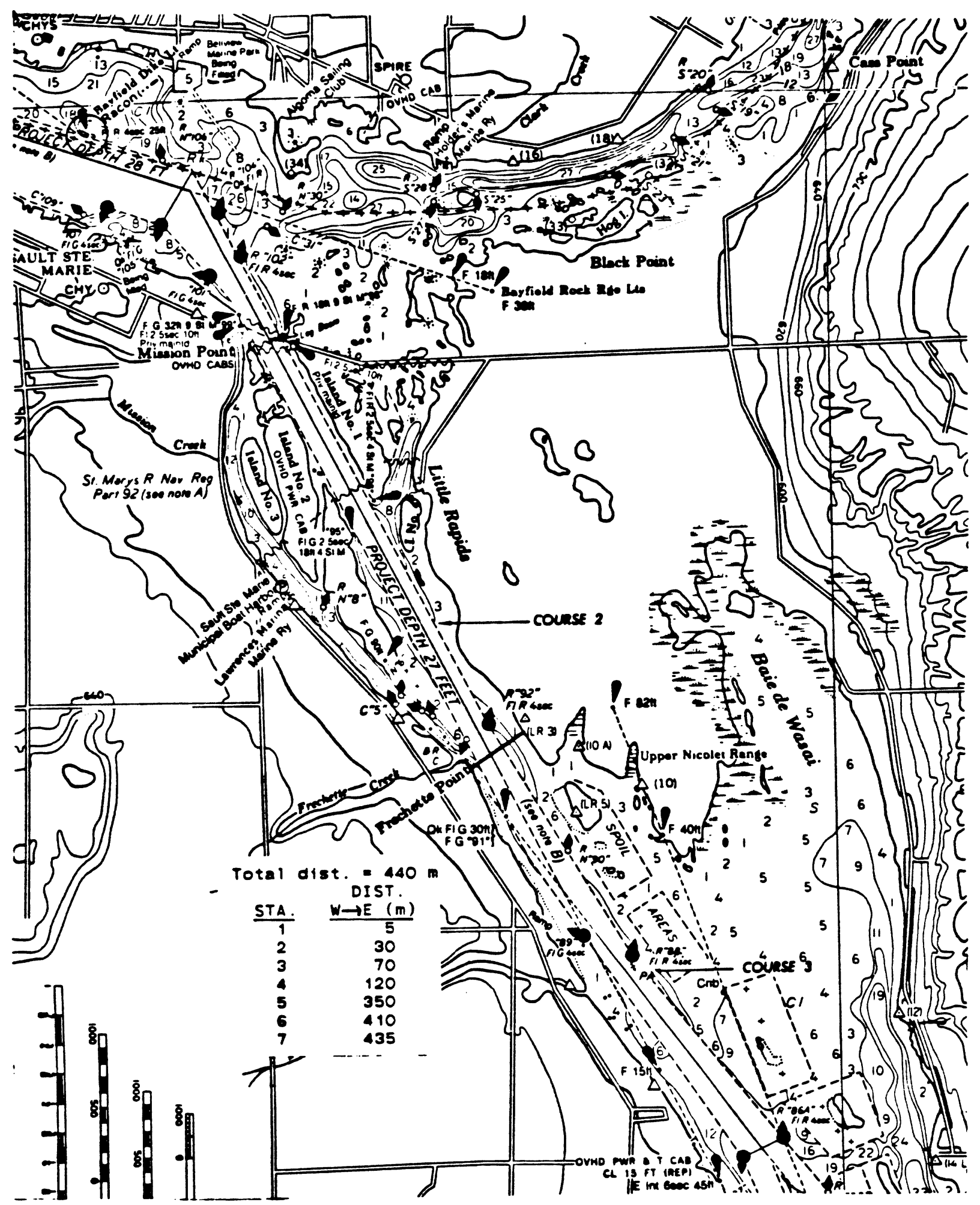

Figure 4. Detailed location maps for the three transects established to sample drift on the St. Marys River, winter and summer, 1985. Taken from NOAA Chart 14883. 


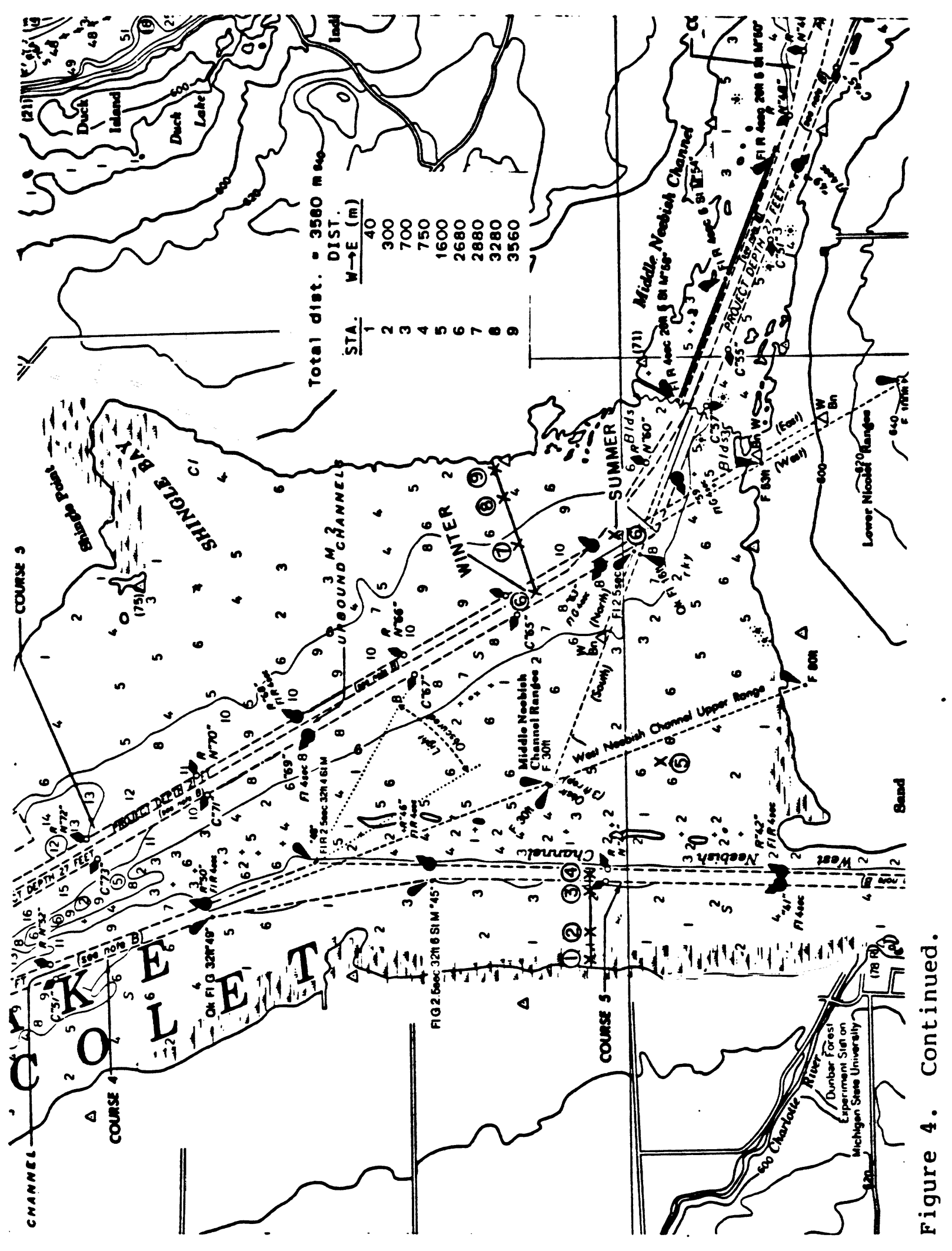




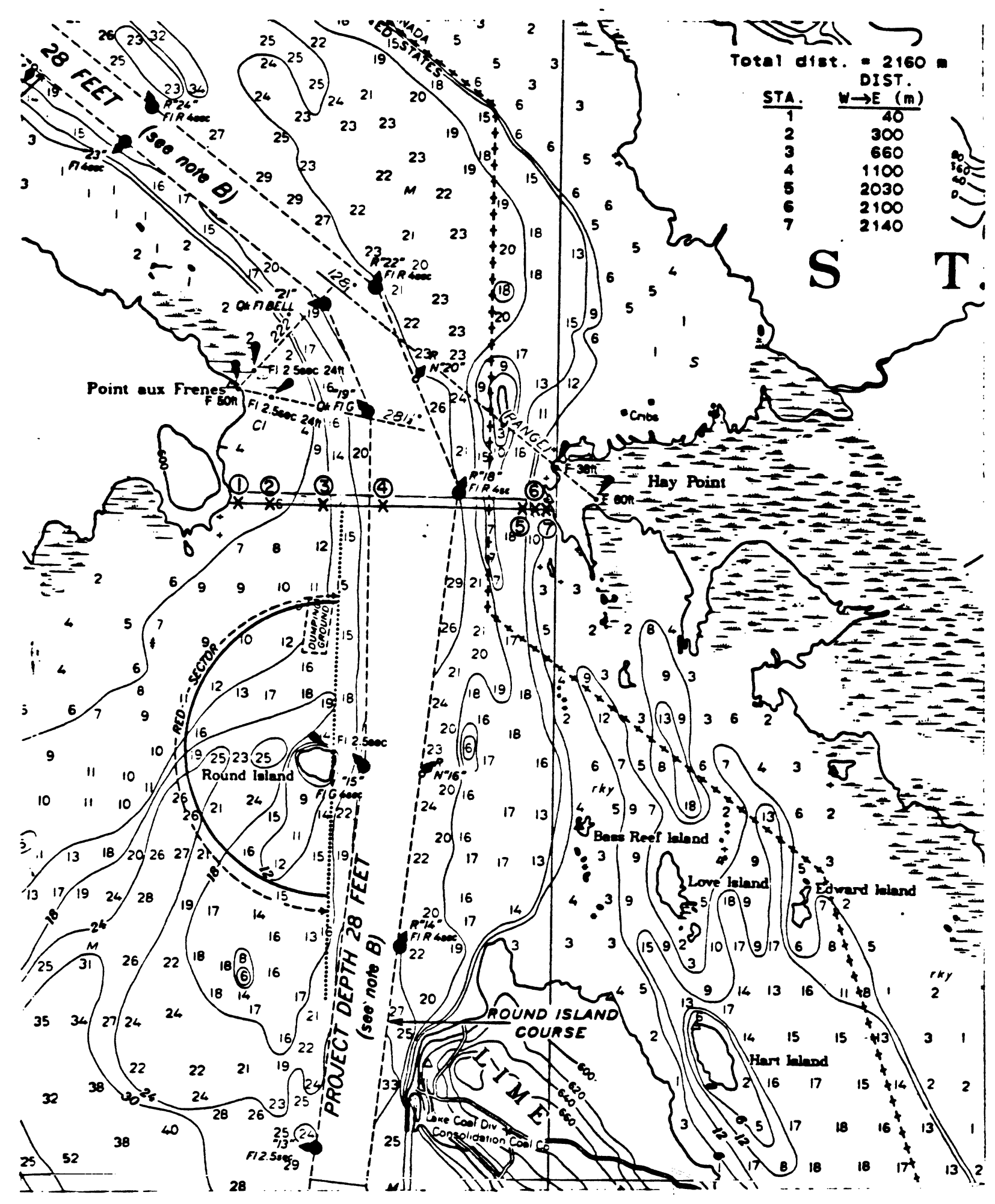

Figure 4. Concluded. 


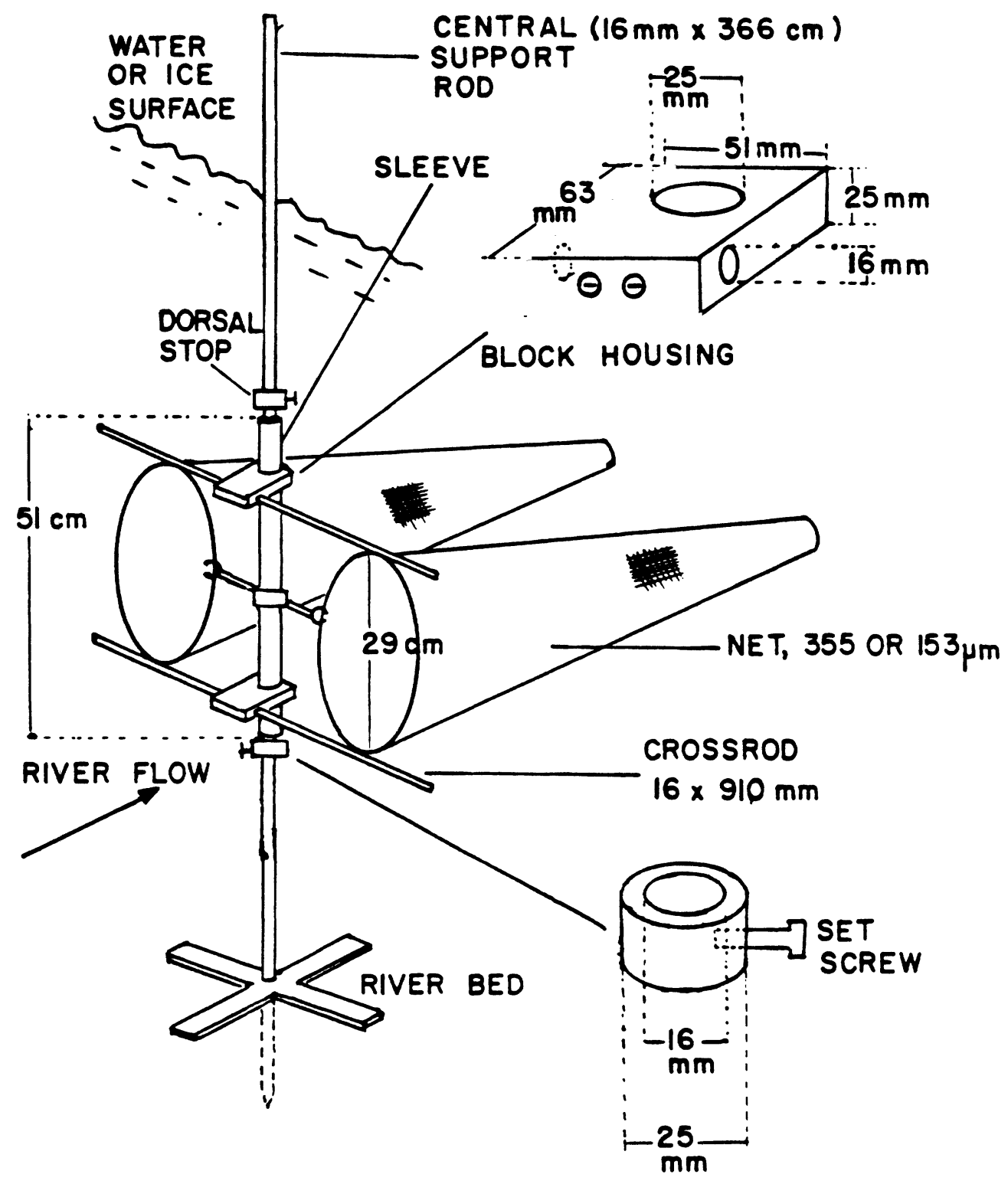

Figure 5. Apparatus used to sample drifting organisms in the St. Marys River. Shown is dual net arrangement mounted on a PVC sleeve which allowed rotation according to current direction. Nets were set without bottom support during the winter and with cross support during the summer. 
and pushed $10-20 \mathrm{~cm}$ into the river bottom. Surface ice acted as support so the pole with nets attached remained upright. During the summer sample period, a $1-$ by $1-m$ base was constructed through which the central, aluminum support rod extended 10-20 $\mathrm{cm}$. When lowered into the water the weight and size of the base as well as the sinking of the rod into the river bed adequately anchored the whole apparatus. At rocky sites, the support was extended 5-10 cm and the base was wedged among the rocks, thereby anchoring the sample device even in rough weather conditions. At the top of each support rod and protruding out of the water was a float with fluorescent markings which functioned to shorten nighttime searches and to warn unsuspecting boaters.

In the navigation channel, the apparatus to which nets were affixed consisted of a 2-cm by 15-m nylon rope, a crossrod, two clips for each pair of replicate nets, and lead weights. Crossrods were stabilized by nylon string supports leading up the nylon rope at $45^{\circ}$ angles from each end of the rod. Each net was clipped at the top and just below center at an angle of $120^{\circ}$. With ice cover, these sets were tied off at the surface to rods laid over the ice hole. In the summer, these sets were tied off to the sides of the 5-m Boston Whaler which was anchored in the channel.

At each sampling location, temperature and water current were determined (see Appendixes 1-7 for raw data). Water current was measured with a Marsh-McBirney Model No. 527 current meter in the winter. However, due to adverse weather conditions which caused the probe to freeze, current was determined during Day 1 sampling periods for all transects except at Frechette Point where several measurements taken indicated little variation in winter current speed. During the summer sample period, a different Marsh-MCBirney current meter failed to operate and was replaced by a Gurley-Fisher current meter.

Adverse weather conditions affected sampling schedules during both winter and summer periods making it impractical to adhere strictly to the original sampling scheme at Point aux Frenes and Lake Nicolet (Table 1). Extremely poor weather conditions ard rough brash ice in the navigation channel prevented collection of 64 winter samples at stations 5 ( 24 samples), 6 (16 samples), and 7 ( 8 samples) at Point aux Frenes and at station 5 ( 16 samples) in lower lake Nicolet. Additionally, these same weather conditions necessitated double setting nets, i.e., setting Day 1 and Day 2 nets on the same day or night period, at stations 1 (Day) and 2 (Night) at Point aux Frenes. During the summer sampling period, high winds and 0.6 - to 1.2-m waves, especially at Point aux Frenes, required the double setting of day and night nets during the same day or night at all stations. 
Table 1. Benthic drift sampling dates for transect stations during winter and summer periods on the St. Marys River. FP = Frechette Point, $L N=$ Lake Nicolet, PAF = Pt. aux Frenes, Surf = sub-surface, Mid = mid-depth, Bott = near-bottom, Trans. = Transect, $\mathbf{S t a}=$ Station, $m=$ meters. A dash means no sample collected.

\begin{tabular}{|c|c|c|c|c|c|c|c|c|c|c|c|c|}
\hline \multirow[b]{2}{*}{ Trans. } & \multirow[b]{2}{*}{ Sta. } & \multicolumn{3}{|c|}{-} & \multirow{2}{*}{\multicolumn{2}{|c|}{$\begin{array}{c}\text { Day } \\
1\end{array}$}} & \multirow{2}{*}{\multicolumn{2}{|c|}{ Night }} & & \multicolumn{3}{|c|}{$-\infty$} \\
\hline & & $\begin{array}{c}\text { Depth } \\
(\mathrm{m})\end{array}$ & $\begin{array}{r}\text { Depth } \\
\text { strata }\end{array}$ & $\begin{array}{c}\text { Mesh } \\
(\mu \mathrm{m})\end{array}$ & & & & & & $\begin{array}{c}\text { Day } \\
2\end{array}$ & & ight \\
\hline$F P$ & 1 & 1.0 & Bott & 355 & 23 & Feb & 23 & Feb & 24 & Feb & 24 & Feb \\
\hline FP & 2 & $\begin{array}{l}1.0 \\
2.0\end{array}$ & $\begin{array}{r}\text { Mid } \\
\text { Bott }\end{array}$ & $\begin{array}{l}355 \\
355\end{array}$ & $\begin{array}{l}23 \\
23\end{array}$ & $\begin{array}{l}\text { Feb } \\
\text { Feb }\end{array}$ & $\begin{array}{l}23 \\
23\end{array}$ & $\begin{array}{l}\text { Feb } \\
\text { Feb }\end{array}$ & $\begin{array}{l}24 \\
24\end{array}$ & $\begin{array}{l}\text { Feb } \\
\text { Feb }\end{array}$ & $\begin{array}{l}24 \\
24\end{array}$ & $\begin{array}{l}\text { Feb } \\
\text { Feb }\end{array}$ \\
\hline FP & 3 & $\begin{array}{l}1.5 \\
3.0 \\
1.5 \\
3.0\end{array}$ & $\begin{array}{l}\text { Mid } \\
\text { Bott } \\
\text { Mid } \\
\text { Bott }\end{array}$ & $\begin{array}{l}355 \\
355 \\
153 \\
153\end{array}$ & $\begin{array}{l}23 \\
23 \\
23 \\
23\end{array}$ & $\begin{array}{l}\text { Feb } \\
\text { Feb } \\
\text { Feb } \\
\text { Feb }\end{array}$ & $\begin{array}{l}23 \\
23 \\
23 \\
23\end{array}$ & $\begin{array}{l}\text { Feb } \\
\text { Feb } \\
\text { Feb } \\
\text { Feb }\end{array}$ & $\begin{array}{l}24 \\
24\end{array}$ & $\begin{array}{r}\text { Feb } \\
\text { Feb } \\
- \\
-\end{array}$ & $\begin{array}{l}24 \\
24\end{array}$ & $\begin{array}{r}\text { Feb } \\
\text { Feb } \\
- \\
-\end{array}$ \\
\hline FP & 4 & $\begin{array}{l}0.5 \\
4.0 \\
8.2 \\
0.5 \\
4.0 \\
8.2\end{array}$ & $\begin{array}{l}\text { Surf } \\
\text { Mid } \\
\text { Bott } \\
\text { Surf } \\
\text { Mid } \\
\text { Bott }\end{array}$ & $\begin{array}{l}355 \\
355 \\
355 \\
153 \\
153 \\
153\end{array}$ & $\begin{array}{l}23 \\
23 \\
23 \\
23 \\
23 \\
23\end{array}$ & $\begin{array}{l}\text { Feb } \\
\text { Feb } \\
\text { Feb } \\
\text { Feb } \\
\text { Feb } \\
\text { Feb }\end{array}$ & $\begin{array}{l}23 \\
23 \\
23 \\
23 \\
23 \\
23\end{array}$ & $\begin{array}{l}\text { Feb } \\
\text { Feb } \\
\text { Feb } \\
\text { Feb } \\
\text { Feb } \\
\text { Feb }\end{array}$ & $\begin{array}{l}24 \\
24 \\
24\end{array}$ & $\begin{array}{r}\text { Feb } \\
\text { Feb } \\
\text { Feb } \\
- \\
- \\
-\end{array}$ & $\begin{array}{l}24 \\
24 \\
24\end{array}$ & $\begin{array}{r}\text { Feb } \\
\text { Feb } \\
\text { Feb } \\
- \\
- \\
-\end{array}$ \\
\hline FP & $\begin{array}{l}5 \\
5\end{array}$ & $\begin{array}{l}1.5 \\
3.0 \\
1.5 \\
3.0\end{array}$ & $\begin{array}{l}\text { Mid } \\
\text { Bott } \\
\text { Mid } \\
\text { Bott }\end{array}$ & $\begin{array}{l}355 \\
355 \\
153 \\
153\end{array}$ & $\begin{array}{l}23 \\
23 \\
23 \\
23\end{array}$ & $\begin{array}{l}\text { Feb } \\
\text { Feb } \\
\text { Feb } \\
\text { Feb }\end{array}$ & $\begin{array}{l}23 \\
23 \\
23 \\
23\end{array}$ & $\begin{array}{l}\text { Feb } \\
\text { Feb } \\
\text { Feb } \\
\text { Feb }\end{array}$ & $\begin{array}{l}24 \\
24\end{array}$ & $\begin{array}{c}\text { Feb } \\
\text { Feb } \\
- \\
-\end{array}$ & $\begin{array}{l}24 \\
24\end{array}$ & $\begin{array}{r}\text { Feb } \\
\text { Feb } \\
- \\
-\end{array}$ \\
\hline FP & 6 & $\begin{array}{l}1.0 \\
2.0\end{array}$ & $\begin{array}{l}\text { Mid } \\
\text { Bott }\end{array}$ & $\begin{array}{l}355 \\
355\end{array}$ & $\begin{array}{l}23 \\
23\end{array}$ & $\begin{array}{l}\text { Feb } \\
\text { Feb }\end{array}$ & $\begin{array}{l}23 \\
23\end{array}$ & $\begin{array}{l}\text { Feb } \\
\text { Feb }\end{array}$ & $\begin{array}{l}24 \\
24\end{array}$ & $\begin{array}{l}\text { Feb } \\
\text { Feb }\end{array}$ & $\begin{array}{l}24 \\
24\end{array}$ & $\begin{array}{l}\text { Feb } \\
\text { Feb }\end{array}$ \\
\hline FP & 7 & 1.0 & Bott & 355 & 23 & Feb & 23 & Feb & 24 & Feb & 24 & Feb \\
\hline LN & 1 & 1.0 & Bott & 355 & 3 & Mar & 3 & Mar & 2 & Mar & 5 & Mar \\
\hline$L N$ & 2 & $\begin{array}{l}1.0 \\
2.0\end{array}$ & $\begin{array}{l}\text { Mid } \\
\text { Bott }\end{array}$ & $\begin{array}{l}355 \\
355\end{array}$ & $\begin{array}{l}3 \\
3\end{array}$ & $\begin{array}{l}\text { Mar } \\
\text { Mar }\end{array}$ & $\begin{array}{l}3 \\
3\end{array}$ & $\begin{array}{l}\text { Mar } \\
\text { Mar }\end{array}$ & $\begin{array}{l}2 \\
2\end{array}$ & $\begin{array}{l}\text { Mar } \\
\text { Mar }\end{array}$ & $\begin{array}{l}5 \\
5\end{array}$ & $\begin{array}{l}\text { Mar } \\
\text { Mar }\end{array}$ \\
\hline$L N$ & $\begin{array}{l}3 \\
3\end{array}$ & $\begin{array}{l}1.5 \\
3.0 \\
1.5 \\
3.0\end{array}$ & $\begin{array}{l}\text { Mid } \\
\text { Bott } \\
\text { Mid } \\
\text { Bott }\end{array}$ & $\begin{array}{l}355 \\
355 \\
153 \\
153\end{array}$ & $\begin{array}{l}3 \\
3 \\
3 \\
3\end{array}$ & $\begin{array}{l}\text { Mar } \\
\text { Mar } \\
\text { Mar } \\
\text { Mar }\end{array}$ & $\begin{array}{l}3 \\
3 \\
3 \\
3\end{array}$ & $\begin{array}{l}\text { Mar } \\
\text { Mar } \\
\text { Mar } \\
\text { Mar }\end{array}$ & $\begin{array}{l}2 \\
2\end{array}$ & $\begin{array}{r}\text { Mar } \\
\text { Mar } \\
- \\
-\end{array}$ & $\begin{array}{l}5 \\
5\end{array}$ & $\begin{array}{r}\text { Mar } \\
\text { Mar } \\
- \\
-\end{array}$ \\
\hline $\mathrm{LN}$ & 4 & $\begin{array}{l}0.5 \\
4.6\end{array}$ & $\begin{array}{r}\text { Surf } \\
\text { Mid }\end{array}$ & $\begin{array}{l}355 \\
355\end{array}$ & $\begin{array}{l}3 \\
3\end{array}$ & $\begin{array}{l}\text { Mar } \\
\text { Mar }\end{array}$ & $\begin{array}{l}3 \\
3\end{array}$ & $\begin{array}{l}\text { Mar } \\
\text { Mar }\end{array}$ & $\begin{array}{l}2 \\
2\end{array}$ & $\begin{array}{l}\text { Mar } \\
\text { Mar }\end{array}$ & $\begin{array}{l}5 \\
5\end{array}$ & $\begin{array}{l}\text { Mar } \\
\text { Mar }\end{array}$ \\
\hline
\end{tabular}


Table 1. (continued)

\begin{tabular}{|c|c|c|c|c|c|c|c|c|c|c|c|c|}
\hline Trans. & Sta. & $\begin{array}{l}\text { Depth } \\
\text { (m) }\end{array}$ & $\begin{array}{r}\text { Depth } \\
\text { strata }\end{array}$ & $\begin{array}{l}\text { Mesh } \\
(\mu \mathrm{m})\end{array}$ & & $\underset{1}{\text { Day }}$ & & ight & & $\begin{array}{c}\text { Day } \\
2\end{array}$ & & ight \\
\hline$(\mathrm{LN})$ & 4 & $\begin{array}{l}9.2 \\
0.5 \\
4.6 \\
9.2\end{array}$ & $\begin{array}{c}\text { Bott } \\
\text { Surf } \\
\text { Mid } \\
\text { Bott }\end{array}$ & $\begin{array}{l}355 \\
153 \\
153 \\
153\end{array}$ & & $\begin{array}{l}\text { Mar } \\
\text { Mar } \\
\text { Mar } \\
\text { Mar }\end{array}$ & & $\begin{array}{l}\text { Mar } \\
\text { Mar } \\
\text { Mar } \\
\text { Mar }\end{array}$ & 2 & $\begin{array}{r}\text { Mar } \\
- \\
- \\
-\end{array}$ & & $\begin{array}{r}\text { Mar } \\
\overline{-} \\
-\end{array}$ \\
\hline LN & 5 & $\begin{array}{l}1.0 \\
2.0\end{array}$ & $\begin{array}{l}\text { Mid } \\
\text { Bott }\end{array}$ & $\begin{array}{l}355 \\
355\end{array}$ & & - & & - & & - & & - \\
\hline LN & 6 & $\begin{array}{l}0.5 \\
4.0 \\
8.2 \\
0.5 \\
4.0 \\
8.2\end{array}$ & $\begin{array}{c}\text { Surf } \\
\text { Mid } \\
\text { Bott } \\
\text { Surf } \\
\text { Mid } \\
\text { Bott }\end{array}$ & $\begin{array}{l}355 \\
355 \\
355 \\
153 \\
153 \\
153\end{array}$ & $\begin{array}{l}6 \\
6 \\
6 \\
6 \\
6 \\
6\end{array}$ & $\begin{array}{l}\text { Mar } \\
\text { Mar } \\
\text { Mar } \\
\text { Mar } \\
\text { Mar } \\
\text { Mar }\end{array}$ & $\begin{array}{l}6 \\
6 \\
6 \\
6 \\
6 \\
6\end{array}$ & $\begin{array}{l}\text { Mar } \\
\text { Mar } \\
\text { Mar } \\
\text { Mar } \\
\text { Mar } \\
\text { Mar }\end{array}$ & $\begin{array}{l}7 \\
7 \\
7\end{array}$ & $\begin{array}{c}\text { Mar } \\
\text { Mar } \\
\text { Mar } \\
- \\
- \\
-\end{array}$ & $\begin{array}{l}7 \\
7 \\
7\end{array}$ & $\begin{array}{r}\text { Mar } \\
\text { Mar } \\
\text { Mar } \\
- \\
- \\
-\end{array}$ \\
\hline LN & $\begin{array}{l}7 \\
7\end{array}$ & $\begin{array}{l}1.5 \\
3.0 \\
1.5 \\
3.0\end{array}$ & $\begin{array}{l}\text { Mid } \\
\text { Bott } \\
\text { Mid } \\
\text { Bott }\end{array}$ & $\begin{array}{l}355 \\
355 \\
153 \\
153\end{array}$ & $\begin{array}{l}6 \\
6 \\
6 \\
6\end{array}$ & $\begin{array}{l}\text { Mar } \\
\text { Mar } \\
\text { Mar } \\
\text { Mar }\end{array}$ & $\begin{array}{l}6 \\
6 \\
6 \\
6\end{array}$ & $\begin{array}{l}\text { Mar } \\
\text { Mar } \\
\text { Mar } \\
\text { Mar }\end{array}$ & $\begin{array}{l}7 \\
7\end{array}$ & $\begin{array}{r}\text { Mar } \\
\text { Mar } \\
- \\
-\end{array}$ & $\begin{array}{l}7 \\
7\end{array}$ & $\begin{array}{r}\text { Mar } \\
\text { Mar } \\
- \\
-\end{array}$ \\
\hline LN & 8 & $\begin{array}{l}1.0 \\
2.0\end{array}$ & $\begin{array}{r}\text { Mid } \\
\text { Bott }\end{array}$ & $\begin{array}{l}355 \\
355\end{array}$ & $\begin{array}{l}6 \\
6\end{array}$ & $\begin{array}{l}\text { Mar } \\
\text { Mar }\end{array}$ & $\begin{array}{l}6 \\
6\end{array}$ & $\begin{array}{l}\text { Mar } \\
\text { Mar }\end{array}$ & $\begin{array}{l}7 \\
7 .\end{array}$ & $\begin{array}{l}\text { Mar } \\
\text { Mar }\end{array}$ & $\begin{array}{l}7 \\
7\end{array}$ & $\begin{array}{l}\text { Mar } \\
\text { Mar }\end{array}$ \\
\hline LN & 9 & 1.0 & Bott & 355 & 6 & Mar & 6 & Mar & 7 & Mar & 7 & Mar \\
\hline PAF & 1 & 1.0 & Bott & 355 & 28 & Feb & 26 & Feb & 28. & Feb & 27 & Feb \\
\hline PAF & 2 & $\begin{array}{l}1.0 \\
2.0\end{array}$ & $\begin{array}{r}\text { Mid } \\
\text { Bott }\end{array}$ & $\begin{array}{l}355 \\
355\end{array}$ & $\begin{array}{l}28 \\
28\end{array}$ & $\begin{array}{l}\text { Feb } \\
\text { Feb }\end{array}$ & $\begin{array}{l}28 \\
28\end{array}$ & $\begin{array}{l}\text { Feb } \\
\text { Feb }\end{array}$ & $\begin{array}{l}27 \\
27\end{array}$ & $\begin{array}{l}\text { Feb } \\
\text { Feb }\end{array}$ & $\begin{array}{l}28 \\
28\end{array}$ & $\begin{array}{l}\text { Feb } \\
\text { Feb }\end{array}$ \\
\hline PAF & 3 & $\begin{array}{l}1.5 \\
3.0 \\
1.5 \\
3.0\end{array}$ & $\begin{array}{l}\text { Mid } \\
\text { Bott } \\
\text { Mid } \\
\text { Bott }\end{array}$ & $\begin{array}{l}355 \\
355 \\
153 \\
153\end{array}$ & $\begin{array}{l}28 \\
28 \\
28 \\
28\end{array}$ & $\begin{array}{l}\text { Feb } \\
\text { Feb } \\
\text { Feb } \\
\text { Feb }\end{array}$ & $\begin{array}{l}26 \\
26 \\
26 \\
26\end{array}$ & $\begin{array}{l}\text { Feb } \\
\text { Feb } \\
\text { Feb } \\
\text { Feb }\end{array}$ & $\begin{array}{l}27 \\
27\end{array}$ & $\begin{array}{c}\text { Feb } \\
\text { Feb } \\
- \\
-\end{array}$ & $\begin{array}{l}27 \\
27\end{array}$ & $\begin{array}{r}\text { Feb } \\
\text { Feb } \\
- \\
-\end{array}$ \\
\hline PAF & 4 & $\begin{array}{l}0.5 \\
4.9 \\
9.8 \\
0.5 \\
4.9 \\
9.8\end{array}$ & $\begin{array}{c}\text { Surf } \\
\text { Mid } \\
\text { Bott } \\
\text { Surf } \\
\text { Mid } \\
\text { Bott }\end{array}$ & $\begin{array}{l}355 \\
355 \\
355 \\
153 \\
153 \\
153\end{array}$ & $\begin{array}{l}27 \\
27 \\
27 \\
27 \\
27 \\
27\end{array}$ & $\begin{array}{l}\text { Feb } \\
\text { Feb } \\
\text { Feb } \\
\text { Feb } \\
\text { Feb } \\
\text { Feb }\end{array}$ & $\begin{array}{l}26 \\
26 \\
26 \\
26 \\
26 \\
26\end{array}$ & $\begin{array}{l}\text { Feb } \\
\text { Feb } \\
\text { Feb } \\
\text { Feb } \\
\text { Feb } \\
\text { Feb }\end{array}$ & $\begin{array}{l}28 \\
28 \\
28\end{array}$ & $\begin{array}{c}\text { Feb } \\
\text { Feb } \\
\text { Feb } \\
- \\
- \\
-\end{array}$ & $\begin{array}{l}27 \\
27 \\
27\end{array}$ & $\begin{array}{r}\text { Feb } \\
\text { Feb } \\
\text { Feb } \\
- \\
-\end{array}$ \\
\hline$F P$ & 1 & 1.0 & Bott & 355 & 2 & Jun & 2 & Jun & 3. & Jun & 3. & Jun \\
\hline
\end{tabular}


Table 1. (continued)

\begin{tabular}{|c|c|c|c|c|c|c|c|c|}
\hline Trans. & Sta. & $\begin{array}{l}\text { Depth } \\
(\mathrm{m})\end{array}$ & $\begin{array}{r}\text { Depth } \\
\text { strata }\end{array}$ & $\begin{array}{c}\text { Mesh } \\
(\mu \mathrm{m})\end{array}$ & $\underset{1}{\text { Day }}$ & Night & $\underset{2}{\text { Day }}$ & Night \\
\hline$F P$ & 2 & $\begin{array}{l}1.0 \\
2.0\end{array}$ & $\begin{array}{l}\text { Mid } \\
\text { Bott }\end{array}$ & $\begin{array}{l}355 \\
355\end{array}$ & $\begin{array}{l}2 \text { Jun } \\
2 \text { Jun }\end{array}$ & $\begin{array}{l}2 \text { Jun } \\
2 \\
\text { Jun }\end{array}$ & $\begin{array}{l}3 \text { Jun } \\
3 \text { Jun }\end{array}$ & $\begin{array}{ll}3 & \text { Jun } \\
3 & \mathrm{Jun}\end{array}$ \\
\hline FP & 3 & $\begin{array}{l}1.5 \\
3.0 \\
1.5\end{array}$ & $\begin{array}{l}\text { Mid } \\
\text { Bott } \\
\text { Mid }\end{array}$ & $\begin{array}{l}355 \\
355 \\
153\end{array}$ & $\begin{array}{ll}2 & \text { Jun } \\
2 & \text { Jun } \\
2 & \text { Jun }\end{array}$ & $\begin{array}{l}2 \text { Jun } \\
2 \text { Jun } \\
2 \text { Jun }\end{array}$ & $\begin{array}{r}3 \text { Jun } \\
3 \text { Jun } \\
-\end{array}$ & $\begin{array}{ll}3 & \text { Jun } \\
3 & \text { Jun } \\
& \end{array}$ \\
\hline FP & 4 & $\begin{array}{l}3.0 \\
0.5 \\
4.6 \\
9.2\end{array}$ & $\begin{array}{l}\text { Bott } \\
\text { Surf } \\
\text { Mid } \\
\text { Bott }\end{array}$ & $\begin{array}{l}153 \\
355 \\
355 \\
355\end{array}$ & $\begin{array}{ll}2 & \text { Jun } \\
2 & \text { Jun } \\
2 & \text { Jun } \\
2 & \text { Jun }\end{array}$ & $\begin{array}{ll}3 & \text { Jun } \\
2 & \text { Jun } \\
2 & \text { Jun } \\
2 & \text { Jun }\end{array}$ & $\begin{array}{ll}3 & - \\
3 & \text { Jun } \\
3 & \text { Jun }\end{array}$ & $\begin{array}{ll}3 & \text { Jun } \\
3 & \text { Jun } \\
3 & \text { Jun }\end{array}$ \\
\hline & 4 & $\begin{array}{l}0.5 \\
4.6 \\
9.2\end{array}$ & $\begin{array}{l}\text { Surf } \\
\text { Mid } \\
\text { Bott }\end{array}$ & $\begin{array}{l}153 \\
153 \\
153\end{array}$ & $\begin{array}{ll}2 & \text { Jun } \\
2 & \mathrm{Jun} \\
2 & \mathrm{Jun}\end{array}$ & $\begin{array}{ll}2 & \text { Jun } \\
2 & \text { Jun } \\
2 & \text { Jun }\end{array}$ & $\overline{-}$ & - \\
\hline FP & 5 & $\begin{array}{l}1.5 \\
3.0\end{array}$ & $\begin{array}{r}\text { Mid } \\
\text { Bott }\end{array}$ & $\begin{array}{l}355 \\
355\end{array}$ & $\begin{array}{l}2 \text { Jun } \\
2 \text { Jun }\end{array}$ & $\begin{array}{l}2 \text { Jun } \\
2 \text { Jun }\end{array}$ & $\begin{array}{l}3 \text { Jun } \\
3 \text { Jun }\end{array}$ & $\begin{array}{l}3 \text { Jun } \\
3 \text { Jun }\end{array}$ \\
\hline & 5 & $\begin{array}{l}1.5 \\
3.0\end{array}$ & $\begin{array}{l}\text { Mid } \\
\text { Bott }\end{array}$ & $\begin{array}{l}153 \\
153\end{array}$ & $\begin{array}{l}2 \text { Jun } \\
2 \text { Jun }\end{array}$ & $\begin{array}{l}2 \text { Jun } \\
2 \text { Jun }\end{array}$ & - & - \\
\hline FP & 6 & $\begin{array}{l}1.0 \\
2.0\end{array}$ & $\begin{array}{r}\text { Mid } \\
\text { Bott }\end{array}$ & $\begin{array}{l}355 \\
355\end{array}$ & $\begin{array}{l}2 \text { Jun } \\
2 \text { Jun }\end{array}$ & $\begin{array}{l}2 \text { Jun } \\
2 \text { Jun }\end{array}$ & $\begin{array}{l}3 \text { Jun } \\
3 \text { Jun }\end{array}$ & $\begin{array}{ll}3 & \mathrm{Jun} \\
3 & \mathrm{Jun}\end{array}$ \\
\hline$F P$ & 7 & 1.0 & Bott & 355 & 2 Jun & 2 Jun & 3 Jun & 3 Jun \\
\hline LN & 1 & 1.0 & Bott & 355 & 5 Jun & 5 Jun & 6 Jun & $6 \mathrm{Jun}$ \\
\hline LN & 2 & $\begin{array}{l}1.0 \\
2.0\end{array}$ & $\begin{array}{r}\text { Mid } \\
\text { Bott }\end{array}$ & $\begin{array}{l}355 \\
355\end{array}$ & $\begin{array}{l}5 \text { Jun } \\
5 \text { Jun }\end{array}$ & $\begin{array}{l}5 \text { Jun } \\
5 \text { Jun }\end{array}$ & $\begin{array}{l}6 \text { Jun } \\
6 \text { Jun }\end{array}$ & $\begin{array}{l}6 \text { Jun } \\
6 \text { Jun }\end{array}$ \\
\hline LN & 3 & $\begin{array}{l}1.5 \\
3.0 \\
1.5 \\
3.0\end{array}$ & $\begin{array}{l}\text { Mid } \\
\text { Bott } \\
\text { Mid } \\
\text { Bott }\end{array}$ & $\begin{array}{l}355 \\
355 \\
153 \\
153\end{array}$ & $\begin{array}{l}5 \text { Jun } \\
5 \text { Jun } \\
5 \text { Jun } \\
5 \text { Jun }\end{array}$ & $\begin{array}{l}5 \text { Jun } \\
5 \text { Jun } \\
5 \text { Jun } \\
5 \text { Jun }\end{array}$ & $\begin{array}{r}6 \text { Jun } \\
6 \text { Jun } \\
- \\
-\end{array}$ & $\begin{array}{ll}6 & \text { Jun } \\
6 & \text { Jun } \\
& \\
& -\end{array}$ \\
\hline LN & 4 & $\begin{array}{l}0.5 \\
4.6 \\
9.2 \\
0.5 \\
4.6 \\
9.2\end{array}$ & $\begin{array}{r}\text { Surf } \\
\text { Mid } \\
\text { Bott } \\
\text { Surf } \\
\text { Mid } \\
\text { Bott }\end{array}$ & $\begin{array}{l}355 \\
355 \\
355 \\
153 \\
153 \\
153\end{array}$ & $\begin{array}{ll}5 & \text { Jun } \\
5 & \text { Jun } \\
5 & \text { Jun } \\
5 & \text { Jun } \\
5 & \text { Jun } \\
5 & \text { Jun }\end{array}$ & $\begin{array}{ll}5 & \text { Jun } \\
5 & \text { Jun } \\
5 & \text { Jun } \\
5 & \text { Jun } \\
5 & \text { Jun } \\
5 & \text { Jun }\end{array}$ & $\begin{array}{r}6 \text { Jun } \\
6 \text { Jun } \\
6 \text { Jun } \\
\quad- \\
- \\
-\end{array}$ & $\begin{array}{lll}6 & \text { Jun } \\
6 & \text { Jun } \\
6 & \text { Jun } \\
& & - \\
& & -\end{array}$ \\
\hline LN & 5 & $\begin{array}{l}1.0 \\
2.0\end{array}$ & $\begin{array}{l}\text { Mid } \\
\text { Bott }\end{array}$ & $\begin{array}{l}355 \\
355\end{array}$ & $\begin{array}{l}5 \text { Jun } \\
5 \text { Jun }\end{array}$ & $\begin{array}{l}5 \text { Jun } \\
5 \text { Jun }\end{array}$ & $\begin{array}{l}6 \text { Jun } \\
6 \text { Jun }\end{array}$ & $\begin{array}{l}6 \text { Jun } \\
6 \text { Jun }\end{array}$ \\
\hline
\end{tabular}


Table 1. Concluded.

\begin{tabular}{|c|c|c|c|c|c|c|c|c|c|c|c|}
\hline Trans. & Sta. & $\begin{array}{l}\text { Depth } \\
(\mathrm{m})\end{array}$ & $\begin{array}{l}\text { Depth } \\
\text { strata }\end{array}$ & $\begin{array}{c}\text { Mesh } \\
(\mu \mathrm{m})\end{array}$ & & $\begin{array}{c}\text { Day } \\
1\end{array}$ & Night & & $\begin{array}{c}\text { Day } \\
2\end{array}$ & & ight \\
\hline LN & 6 & $\begin{array}{l}0.5 \\
4.6 \\
9.2 \\
0.5 \\
4.6 \\
9.2\end{array}$ & $\begin{array}{l}\text { Surf } \\
\text { Mid } \\
\text { Bott } \\
\text { Surf } \\
\text { Mid } \\
\text { Bott }\end{array}$ & $\begin{array}{l}355 \\
355 \\
355 \\
153 \\
153 \\
153\end{array}$ & $\begin{array}{l}6 \\
6 \\
6 \\
6 \\
6 \\
6\end{array}$ & $\begin{array}{l}\text { Jun } \\
\text { Jun } \\
\text { Jun } \\
\text { Jun } \\
\text { Jun } \\
\text { Jun }\end{array}$ & $\begin{array}{l}6 . \mathrm{Jun} \\
6 \text { Jun } \\
6 \text { Jun } \\
6 \text { Jun } \\
6 \text { Jun } \\
6 \text { Jun }\end{array}$ & $\begin{array}{l}7 \\
7 \\
7\end{array}$ & $\begin{array}{c}\text { Jun } \\
\text { Jun } \\
\text { Jun } \\
- \\
- \\
-\end{array}$ & $\begin{array}{l}7 \\
7 \\
7\end{array}$ & $\begin{array}{c}\text { Jun } \\
\text { Jun } \\
\text { Jun } \\
- \\
- \\
-\end{array}$ \\
\hline$L N$ & $\begin{array}{l}7 \\
7\end{array}$ & $\begin{array}{l}1.5 \\
3.0 \\
1.5 \\
3.0\end{array}$ & $\begin{array}{l}\text { Mid } \\
\text { Bott } \\
\text { Mid } \\
\text { Bott }\end{array}$ & $\begin{array}{l}355 \\
355 \\
153 \\
153\end{array}$ & $\begin{array}{l}7 \\
7 \\
7 \\
7\end{array}$ & $\begin{array}{l}\text { Jun } \\
\text { Jun } \\
\text { Jun } \\
\text { Jun }\end{array}$ & $\begin{array}{ll}7 & \text { Jun } \\
7 & \mathrm{Jun} \\
7 & \mathrm{Jun} \\
7 & \mathrm{Jun}\end{array}$ & $\begin{array}{l}8 \\
8\end{array}$ & $\begin{array}{c}\text { Jun } \\
\text { Jun } \\
- \\
-\end{array}$ & $\begin{array}{l}8 \\
8\end{array}$ & $\begin{array}{r}\text { Jun } \\
\text { Jun } \\
- \\
-\end{array}$ \\
\hline $\mathrm{LN}$ & 8 & $\begin{array}{l}1.0 \\
2.0\end{array}$ & $\begin{array}{l}\text { Mid } \\
\text { Bott }\end{array}$ & $\begin{array}{l}355 \\
355\end{array}$ & & $\begin{array}{l}\text { Jun } \\
\text { Jun }\end{array}$ & $\begin{array}{l}7 \text { Jun } \\
7 \text { Jun }\end{array}$ & $\begin{array}{l}8 \\
8\end{array}$ & $\begin{array}{l}\text { Jun } \\
\text { Jun }\end{array}$ & $\begin{array}{l}8 \\
8\end{array}$ & $\begin{array}{l}\text { Jun } \\
\text { Jun }\end{array}$ \\
\hline LN & 9 & 1.0 & Mid & 355 & 7 & Jun & $7 \mathrm{Jun}$ & 8 & Jun & 8 & Jun \\
\hline PAF & 1 & 1.0 & Bott & 355 & 11 & Jun & 11 Jun & 12 & Jun & 11 & Jun \\
\hline PAF & 2 & $\begin{array}{l}1.0 \\
2.0\end{array}$ & $\begin{array}{l}\text { Mid } \\
\text { Bott }\end{array}$ & $\begin{array}{l}355 \\
355\end{array}$ & $\begin{array}{l}11 \\
11\end{array}$ & $\begin{array}{l}\text { Jun } \\
\text { Jun }\end{array}$ & $\begin{array}{l}11 \text { Jun } \\
11 \text { Jun }\end{array}$ & $\begin{array}{l}12 \\
12\end{array}$ & $\begin{array}{l}\text { Jun } \\
\text { Jun }\end{array}$ & $\begin{array}{l}11 \\
11\end{array}$ & $\begin{array}{l}\text { Jun } \\
\text { Jun }\end{array}$ \\
\hline PAF & $\begin{array}{l}3 \\
3\end{array}$ & $\begin{array}{l}1.5 \\
3.0 \\
1.5 \\
3.0\end{array}$ & $\begin{array}{l}\text { Mid } \\
\text { Bott } \\
\text { Mid } \\
\text { Bott }\end{array}$ & $\begin{array}{l}355 \\
355 \\
153 \\
153\end{array}$ & $\begin{array}{l}11 \\
11 \\
11 \\
11\end{array}$ & $\begin{array}{l}\text { Jun } \\
\text { Jun } \\
\text { Jun } \\
\text { Jun }\end{array}$ & $\begin{array}{ll}11 & \text { Jun } \\
11 & \mathrm{Jun} \\
11 & \mathrm{Jun} \\
11 & \mathrm{Jun}\end{array}$ & $\begin{array}{l}12 \\
12\end{array}$ & $\begin{array}{c}\text { Jun } \\
\text { Jun } \\
- \\
-\end{array}$ & $\begin{array}{l}11 \\
11\end{array}$ & $\begin{array}{r}\text { Jun } \\
\text { Jun } \\
- \\
-\end{array}$ \\
\hline PAF & 4 & $\begin{array}{l}0.5 \\
4.6 \\
9.2 \\
0.5 \\
4.6 \\
9.2\end{array}$ & $\begin{array}{l}\text { Surf } \\
\text { Mid } \\
\text { Bott } \\
\text { Surf } \\
\text { Mid } \\
\text { fott }\end{array}$ & $\begin{array}{l}355 \\
355 \\
355 \\
153 \\
153 \\
153\end{array}$ & $\begin{array}{l}13 \\
13 \\
13 \\
13 \\
13 \\
13\end{array}$ & $\begin{array}{l}\text { Jun } \\
\text { Jun } \\
\text { Jun } \\
\text { Jun } \\
\text { Jun } \\
\text { Jun }\end{array}$ & $\begin{array}{ll}14 & \text { Jun } \\
14 & \text { Jun } \\
14 & \text { Jun } \\
14 & \text { Jun } \\
14 & \text { Jun } \\
14 & \text { Jun }\end{array}$ & $\begin{array}{l}13 \\
13 \\
13\end{array}$ & $\begin{array}{c}\text { Jun } \\
\text { Jun } \\
\text { Jun } \\
- \\
- \\
-\end{array}$ & $\begin{array}{l}14 \\
14 \\
14\end{array}$ & $\begin{array}{c}\text { Jun } \\
\text { Jun } \\
\text { Jun } \\
- \\
- \\
-\end{array}$ \\
\hline PAF & $\begin{array}{l}5 \\
5\end{array}$ & $\begin{array}{l}1.5 \\
3.0 \\
1.5 \\
3.0\end{array}$ & $\begin{array}{l}\text { Mid } \\
\text { Bott } \\
\text { Mid } \\
\text { Bott }\end{array}$ & $\begin{array}{l}355 \\
355 \\
153 \\
153\end{array}$ & $\begin{array}{l}12 \\
12 \\
12 \\
12\end{array}$ & $\begin{array}{l}\text { Jun } \\
\text { Jun } \\
\text { Jun } \\
\text { Jun }\end{array}$ & $\begin{array}{l}13 \text { Jun } \\
13 \text { ปun } \\
13 \text { Jun } \\
13 \text { Jun }\end{array}$ & $\begin{array}{l}12 \\
12\end{array}$ & $\begin{array}{r}\text { Jun } \\
\text { Jun } \\
- \\
-\end{array}$ & $\begin{array}{l}13 \\
13\end{array}$ & $\begin{array}{r}\text { Jun } \\
\text { Jun } \\
- \\
-\end{array}$ \\
\hline PAF & 6 & $\begin{array}{l}1.0 \\
2.0\end{array}$ & $\begin{array}{l}\text { Mid } \\
\text { Bott }\end{array}$ & $\begin{array}{l}355 \\
355\end{array}$ & $\begin{array}{l}12 \\
12\end{array}$ & $\begin{array}{l}\text { Jurı } \\
\text { Jurı }\end{array}$ & 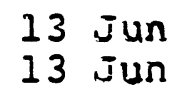 & $\begin{array}{l}12 \\
12\end{array}$ & $\begin{array}{l}\text { Jun } \\
\text { Jun }\end{array}$ & $\begin{array}{l}13 \\
13\end{array}$ & $\begin{array}{l}\text { Jun } \\
\text { Jun }\end{array}$ \\
\hline PAF & 7 & 1.0 & Bott & 355 & 12 & Jun & 13 Jun & 12 & Jun & 13 & Jun \\
\hline
\end{tabular}


To compensate for the uncollected winter samples, a special summer study was devised which required the collection of 40 drift samples. The purpose of the study was to estimate the impact on drift of upbound and downbound passage of ships in excess of $213 \mathrm{~m}$ in length. This study was conducted during daylight hours on 10 June 1985 at station 3 at Frechette Point. Sampling was conducted in the same fashion as all previous drift sampling at Frechette Point except sampling was centered around passage of a ship using 153-um mesh nets only. Replicate samples were collected at mid-depth and near-bottom during five periods corresponding to before ship passage, during ship passage, $5 \mathrm{~min}$ after ship passage, $10 \mathrm{~min}$ after ship passage, and $15 \mathrm{~min}$ after ship passage. Each period was approximately $5 \mathrm{~min}$ in duration. Between periods there was a 2-3 min lag during which nets were retrieved and reset. Current speed was monitored for the "before" period and continuously in I-min intervals for the "during" period, but no current measurements were taken during the "after" periods. Additionally, for each ship, length, width, and draft were noted. Ship speed was estimated by timing ship passage from bow to stern past a fixed point directly opposite station 3. Utilizing this scheme, 20 samples were collected for one upbound and one downbound ship passage.

All net contents were washed into labeled, 0.5-L Mason jars, preserved in $4 \%$ formaldehyde, and returned to the Benthos Laboratory. The collected drift was examined at 3-30x using stereo-zoom dissecting microscopes. Benthos, $f$ ish larvae, and $f$ ish eggs in the drift were identified and enumerated, with each component placed in labeled vials. All macrophytic material was removed and placed in labeled vials for identification in the Botany Laboratory. Remaining contents of each drift sample were again placed in $4 \%$ formaldehyde and taken to the Zooplankton Laboratory (Great Lakes Research Division) where final processing occurred. All benthos, fish larvae and eggs, and macrophytic vials were taken to the Zooplankton Laboratory for dry weight and ash-free dry weight analyses.

All data initially recorded on raw data sheets stored in the Benthos Laboratory were coded and entered into the University of Michigan's AMDAHL computer. All analyses were conducted using the Michigan Interactive Data Analysis System (MIDAS) which is a system of statistical analysis programs supported by the Statistical Research Laboratory at the University of Michigan. Comparisons of drift density differences were based on $\log _{10}(x+1)$ transformations of numbers per $1,000 \mathrm{~m}^{3}$ density estimates to better approximate normality. Scheffe multiple comparisons ( $a=$ 0.05 ) (Sokal and Rohlf 1969) were used to evaluate benthic and larval $f$ ish drift density differences among net types, transects, stations, depth strata, diel periods, and components of the ship passage study. 
Drift rates were calculated for day and night periods during winter and summer at Frechette Point and Lake Nicolet. At Point aux Frenes, estimates were made only for the summer, because samples were not collected for the entire transect during winter. At each transect, the river cross-section was divided vertically (depth strata) and horizontally (stations). The area of each resultant subsection was estimated by station distance from shore and station depth. The area between adjacent stations was divided equally. The upper $0.5 \mathrm{~m}$ of the river cross-section was considered the surface depth stratum. The mid-depth stratum extended from $0.5 \mathrm{~m}$ deep to midway between the mid-depth net and the bottom net location. The remaining vertical portion of the river was assigned to the bottom depth stratum. Based on these areal approximations and by calculating an average current velocity and drift density for day and night periods at each river subsection during a given season, we estimated seasonal drift rate passing each of our transects (number of individuals/ $24 \mathrm{hr}$ ) by summing day and night estimates derived from the following equation:

Drift rate $=$

$(\mathrm{cm} / \mathrm{s})(3600 \mathrm{~s} / \mathrm{h})(\mathrm{h} / \mathrm{d})\left(\mathrm{m}^{2}\right)(\mathrm{m} / 100 \mathrm{~cm}) \overline{\mathrm{x}}$ density/l,000 $\left.\mathrm{m}^{3}\right)$

During winter the daylight period was assumed to be $11 \mathrm{~h}$ and the night time was $13 \mathrm{~h}$. During the summer the daylight period was $15 \mathrm{~h}$ and the night was $9 \mathrm{~h}$ in duration. Our discharge estimate $\left(\mathrm{m}^{3} / 24 \mathrm{~h}\right)$ was calculated by removing the last term in the equation $\left(\bar{x}\right.$ density/l,000 $\left.\mathrm{m}^{3}\right)$. Total drift rate and discharge passing each transect was calculated by summing appropriate values across all subsections and time periods within each season. Based on these sums, drift intensity was calculated from the total number of individuals passing per 24-h period divided by discharge expressed as $\mathrm{m}^{3} / \mathrm{s}$ (Waters 1972).

\section{ZOOPLANKTON}

\section{Identifications}

Zooplankton in samples were identified to either genus or species. At most stations, zooplankton were identified to no higher than genus level. However, species identifications were conducted at station 3 at all three sampling transects.

For those collections where zooplankton were identified to the genus level, each sample was subdivided as many times as necessary in a Folsom plankton splitter to yield two subsamples of 200-250 animals each. For collections where zooplankton were identified to species, each sample was subdivided to yield two subsamples of 100-150 animals each. Both subsamples were examined. 
Zooplankton were examined in a circular counting dish under a Wild Stereozoom M8 microscope. Immature copepodites and cladocerans were identified to genus, while nauplii were combined as a group. Adult copepods and cladocerans were identified to species for station 3 samples collected during day 1 and night 1 ; sex determinations were made for adult copepods. At all other stations, identifications were to genus level. With the exception of Asplanchna, rotifers were not enumerated or identified. All data were entered on coding sheets.

\section{Biomass Determinations}

Samples received in the Zooplankton Laboratory were split into three groups: plants, benthos, and $f$ ish larvae and $f$ ish eggs. Organisms were sorted and placed in separate labelled vials within the original sample jar. No fish larvae were collected during winter.

Dry weights and ash-free weights were determined for benthos, plants, and seston. The basic procedures were as follows.

\section{Benthos--}

For most benthic samples, organisms were removed from the vial and washed several times in a petri dish of distilled water. All work was done under a Zeiss binocular microscope. Animals were enumerated and placed in preweighed 4-mm $\times 13-\mathrm{mm}$ aluminum boats manufactured by Hewlett Packard. Boats were individually placed on numbered $26-\mathrm{mm}$ diameter aluminum discs and dried in a desiccator (containing silica gel) over 2 days at room temperature. After 2 days, boats were reweighed on a Cahn electrobalance. The difference between the original and final weigh provided an estimate of the weight of benthic organisms in the original sample.

The boats and numbered discs were then placed in a muffle furnace and burned for $2 \mathrm{~h}$ at $450-500^{\circ} \mathrm{C}$. After cooling, the boats and numbered discs were removed from the furnace and reweighed on the electrobalance. The difference in weight after drying in the desiccator and burning in the furnace provided an estimate of the ash-free weight of benthos in the original sample.

On occasion, samples contained large numbers of benthos. In this case, weighing procedures for seston (see below) were followed. 
When small amounts of plant material were present, vial contents were filtered on a prewashed and preashed 2.4-cm Whatman glass fiber filter. The sample was then washed several times with distilled water, placed on a numbered 2.6-cm diameter aluminum disc, and dried for 2 days at $55^{\circ} \mathrm{C}$ in an oven. The filter was then reweighed on a Cahn electrobalance, placed back on its numbered disc, and burned in the muffle furnace. After cooling, the ashed sample was reweighed.

In practice, many plant samples contained small fragments of plant material which would better be designated as detritus. Thus, we defined plant material as larger, more intact plants and fragments.

In some cases, large amounts of plant material were present in samples. In these cases, plant material was removed, washed, and placed directly on preweighed, 5.0-cm diameter, numbered aluminum discs. Samples were desiccated for 2 days in the drying oven, reweighed, and then ashed in the muffle furnace for $2 \mathrm{~h}$ and reweighed. Most weighings were done on a Mettler balance.

Seston--

Due to the large amount of material present in most samples, only subsamples of seston were weighed. During the subsampling procedure for zooplankton identifications, one subsample containing 600-1,000 animals was retained and placed in a labelled vial. This seston subsample was subsequently filtered with a 2.4-cm prewashed and preashed glass fiber filter. The sample was washed several times with distilled water and placed on a numbered $3.2-\mathrm{cm}$ diameter aluminum disc. It was dried at $55^{\circ} \mathrm{C}$ for 2 days in an oven, then the filter reweighed. The filter was placed back on its numbered disc and burned for 2 hours in the muffle furnace prior to final reweighing.

\section{Zooplankton--}

Individual zooplankton dry weights were determined using the following procedure. Numerically dominant taxa were identified and samples selected for biomass determinations. Approximately 10-50 animals of each taxon were removed from a sample, washed, and placed on preweighed, circular 5-mm diameter aluminum boats. The boats were placed on numbered 26-mm diameter aluminum discs, dried at room temperature for 2 days in a desiccator, and reweighed. Ash-free weights were based on literature conversion values. 
Weather conditions were coded into $1=c a l m, 2=$ windy, and 3 = very windy. These values were then averaged to give a quantitative estimate of weather conditions to relate to drift results.

\section{FISH LARVAE}

\section{Study Area}

Seven larval fish sampling stations were established on the St. Marys River. Station I was located at the railroad bridge on the Sault Edison Hydropower Canal in Sault Ste. Marie (Table 2, Fig. 6). We sampled on the east side of the bridge between the first and second support columns.

Station 2 was near buoy 86A off Six Mile Point on the east side of the river. Substrate was sand, gravel, and cobble.

Station 3 (buoy 53) was located in the west (downbound) channel where the channel splits around the northern tip of Neebish Island. The channel was $1.1 \mathrm{~km}$ from the mainland, and onshore cattail stands predominated. Substrate was mostly firm, though occasionally soft, and appeared to be organic material; no rocks were noted.

Station 4 (buoy 72) was located in the east channel upstream from Neebish Island. At this station the channel was about 1.3 $\mathrm{km}$ from Sugar Island. Substrate was mostly soft, and included organic material and sand. Cattails predominated along the shoreline.

Station 5 (buoy 62) was located off the southwestern tip of Sugar Island. It was the most unique station, being very rocky with large boulders common alongshore. This station had an undulating contour with large boulders projecting from the water up to $100 \mathrm{~m}$ from shore.

Station 6 (buoy 33) was located in the upbound channel on the east side of Neebish Island. The bottom was sand, with the adjacent shoreline comprised of an extensive wooded swamp. Scattered organic debris (sticks, tree branches) was sometimes encountered.

Station 7 was at buoy 13. This station was at the southern tip of Neebish Island, east of Winter Point, near a dump site (rocks, dredge spoils) which forms a spit in the river and a bay behind the dump site. The shore was wooded and the boitom firm and sandy with some organic material. The entire shoreline appeared to be similar from winter Foint to the spit. At stations 2 through 7 , channel sampling was conducted between the 


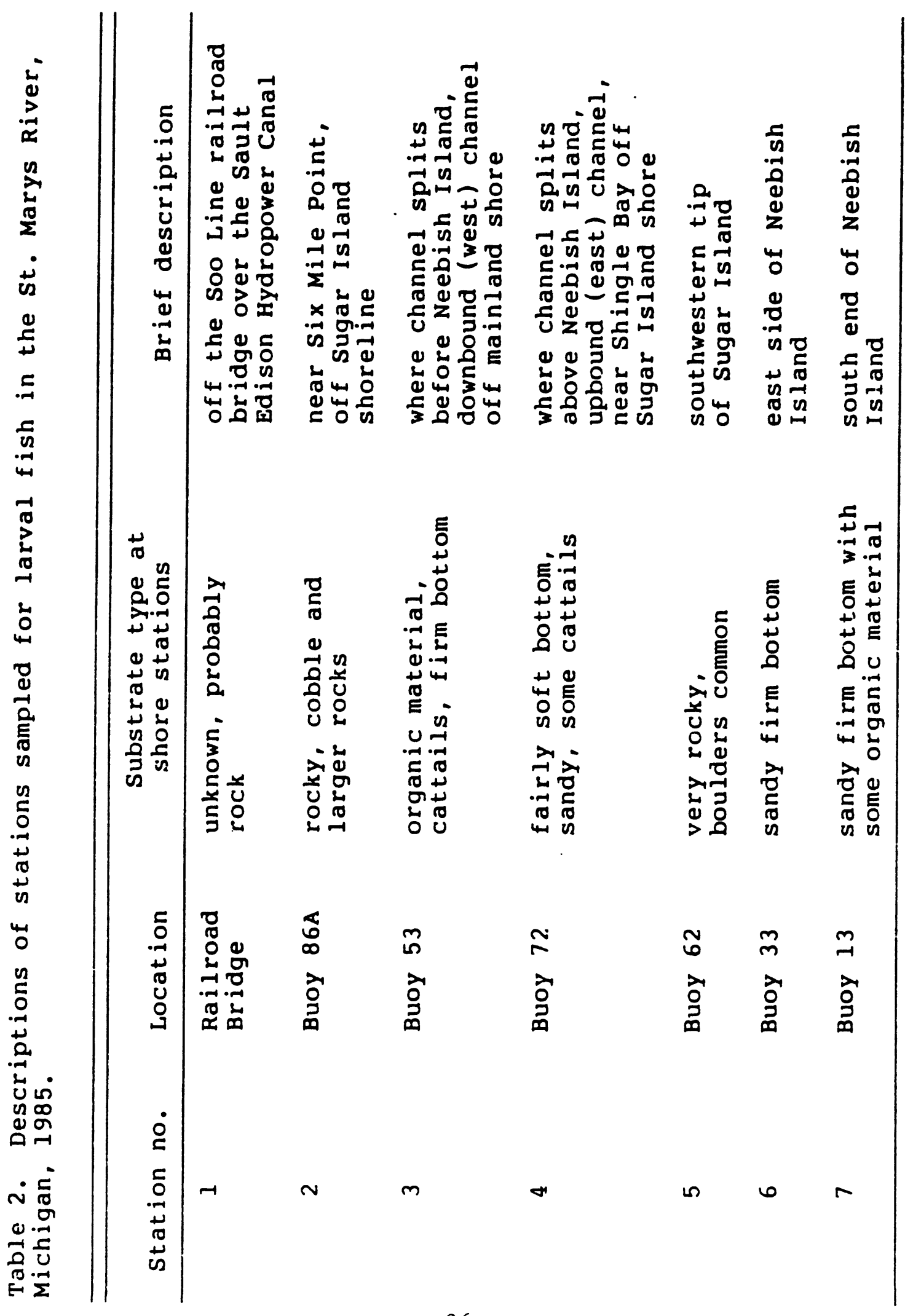




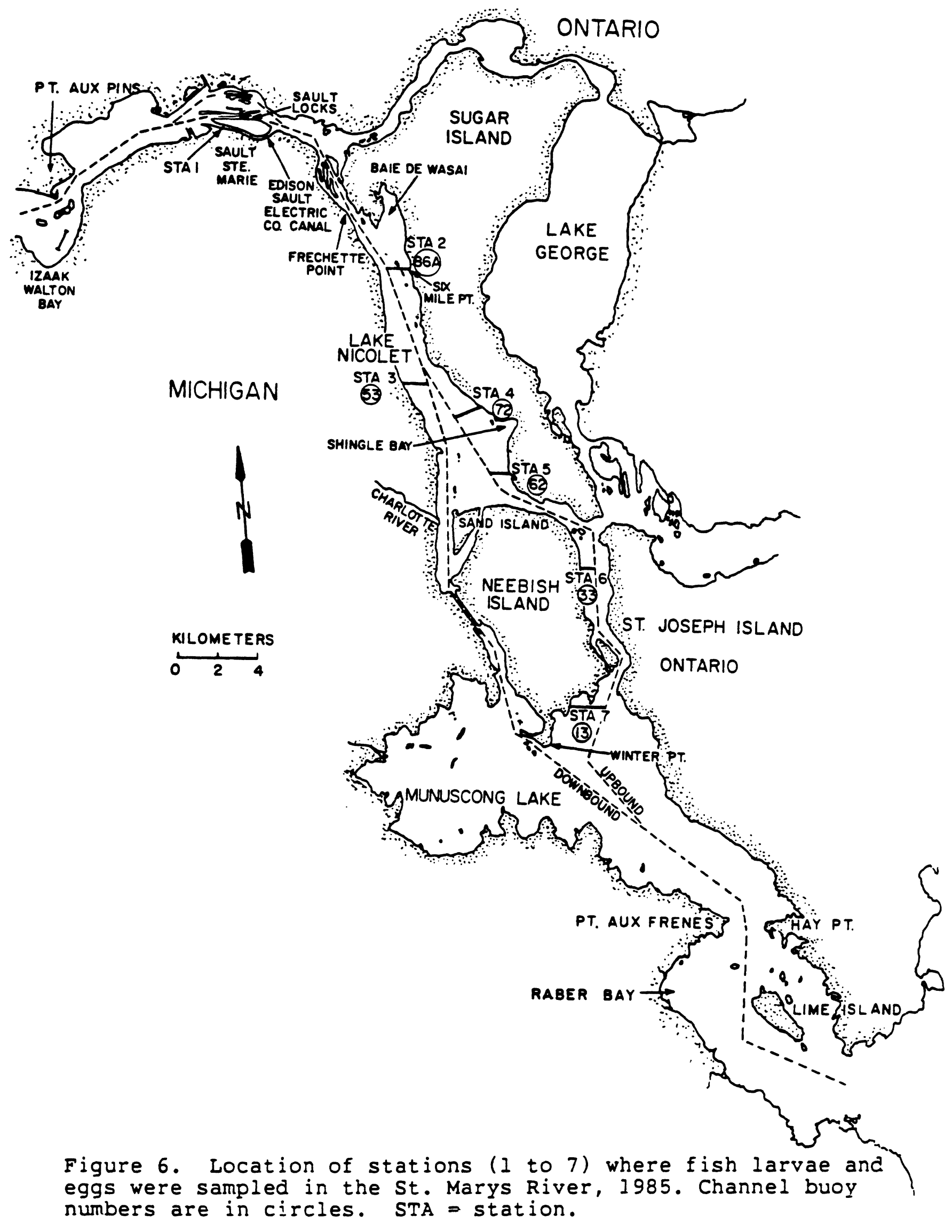


9- and 11-m contours (in the shipping channel), while nearshore samples were taken at the 1 - and $2-m$ depth contours, along the shore adjacent to the buoy marking the station.

\section{Sampling Procedures}

Bridge Samples--

At the railroad bridge we collected duplicate night samples with a $0.5-\mathrm{m}$ diameter, 363-um mesh net. The net was secured to the bridge with rope and sampled $\leq 1 \mathrm{~m}$ below the surface for 10 min. Surface water temperature and current velocity were measured (Figs. 7 and 8 ). During the first week, samples were collected with a l-m diameter net, but because of the difficulty in retrieving the net against the strong current, we switched to a $0.5-m$ diameter net for all remaining samples.

\section{Shore Pull Net Samples--}

A specially designed pull net (Fig. 9), equipped with

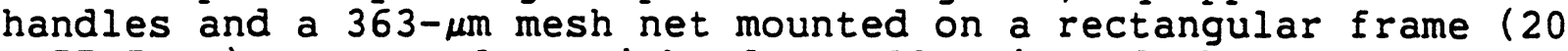
$X 57.5 \mathrm{~cm}$ ), was used at night for collection of shore samples at each station. The net was equipped with a flowmeter and was pulled by two people walking in about $1 \mathrm{~m}$ of water. The net usually was in mid-depth and the two people were about $4 \mathrm{~m}$ apart. They walked upstream as fast as possible for 66 paces or approximately $61 \mathrm{~m}$. Duplicate, non-overlapping samples were collected. Water temperature was measured at each station for all samples.

Push Net Samples--

Push net samples ( $F$ ig. 10) were collected using a $0.5-\mathrm{m}$ diameter, 363-um mesh net rigidly mounted in front of a $4.9-\mathrm{m}$ Boston Whaler; duplicate tows were performed at about $0.5 \mathrm{~m}$ below the surface of the water. We ran the boat engine (70-hp Evinrude) at 1,000 RPM upriver for $5 \mathrm{~min}$ at the 2-m depth contour.

\section{Channel Samples--}

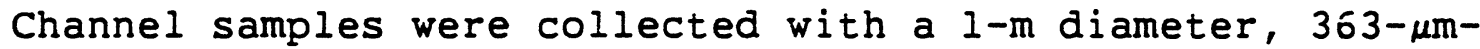
mesh net; duplicate, stepped oblique tows were performed (Fig. 1l). We standardized the tows so that the net $f$ ished at four depths (surface, $3 \mathrm{~m}, 5 \mathrm{~m}$, and $7 \mathrm{~m}$ ) for $1 \mathrm{~min}$ each, with $15 \mathrm{~s}$ between each depth as the net was retrieved. The engine was run at 1,500 RPM. 


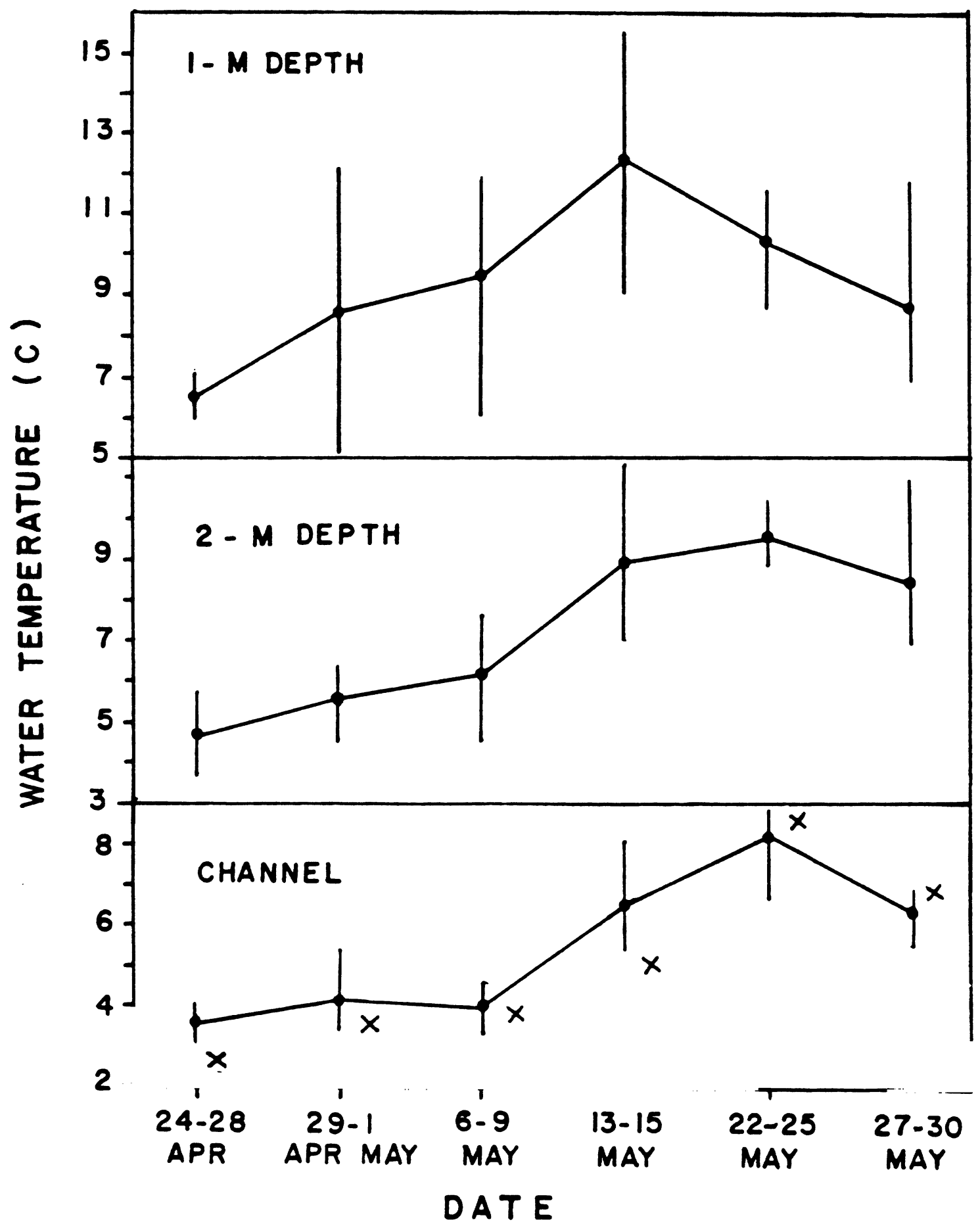

Figure 7. Mean and range (vertical lines) of bottom water temperatures measured at six stations in the st. Marys River, 1985. X's designated surface water temperature in the Edison Hydropower Canal - station 1. 


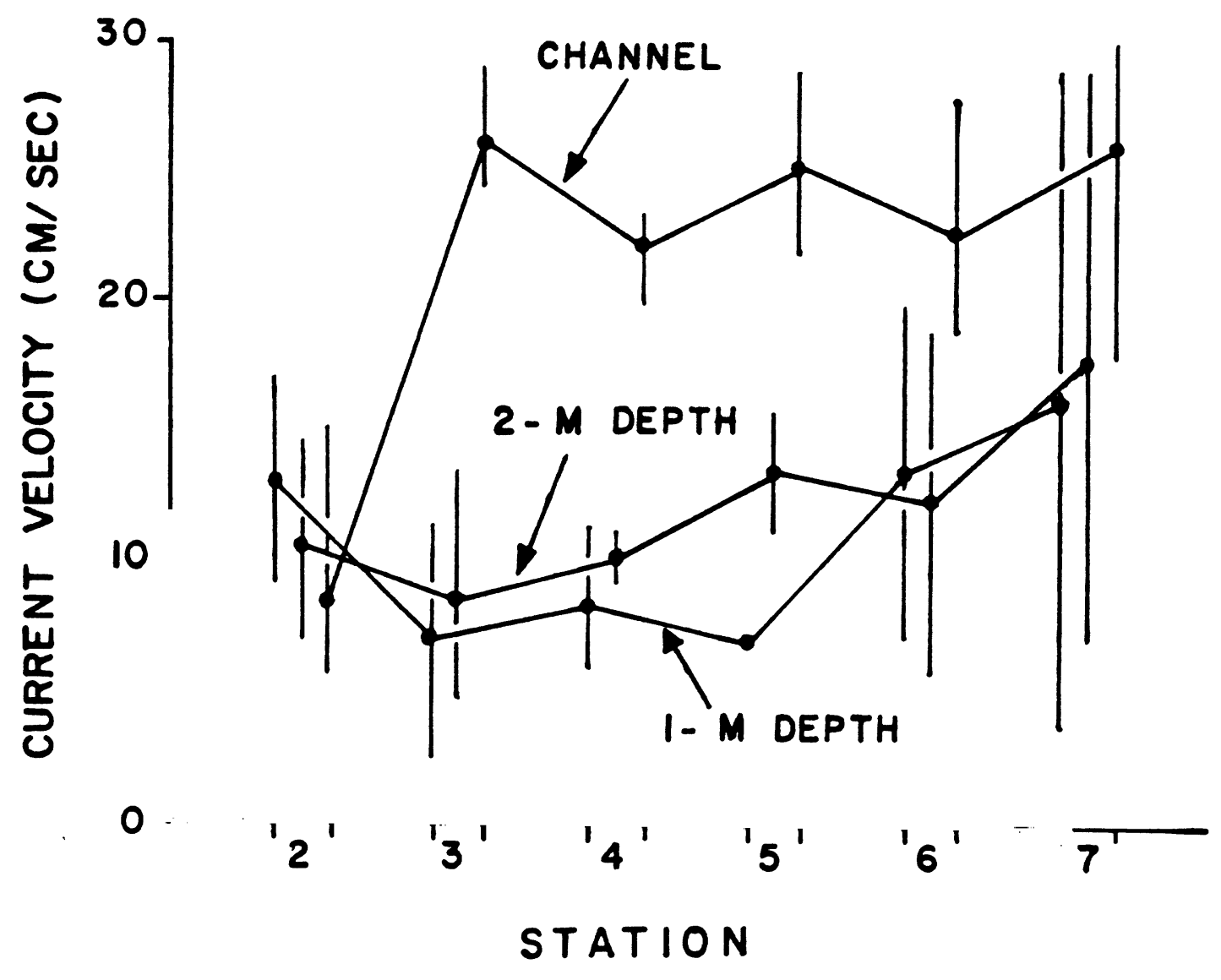

Figure 8. Current velocities measured at six stations in the St. Marys River, 1985. Points indicate means; vertical lines represent ranges; $N$ varied from 2 to 5 . 


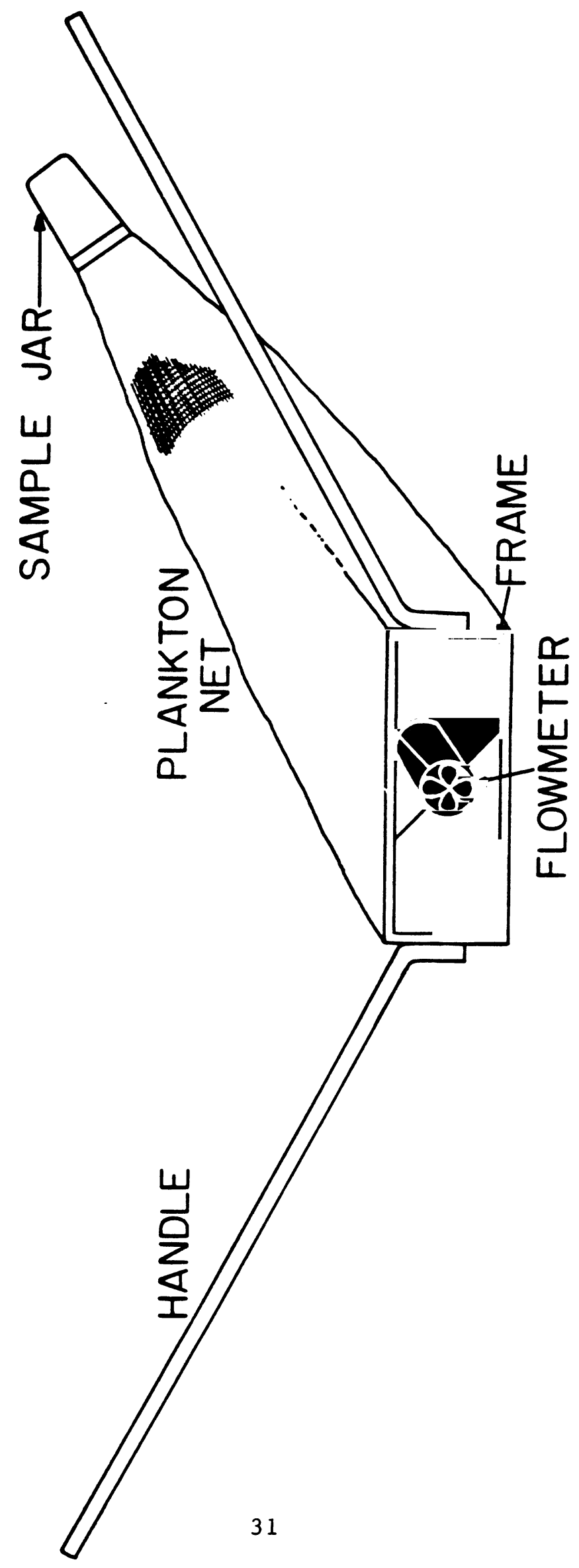

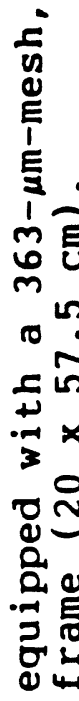

ต

כ

ฉ

茴

is

ถุ

$+\pi$

든

일

ติ

เั

次

넌

E

ס

ขิ

ข엄

茂

(1)

$\rightarrow$

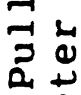

口

ต.

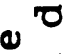

는

ชั

जि 


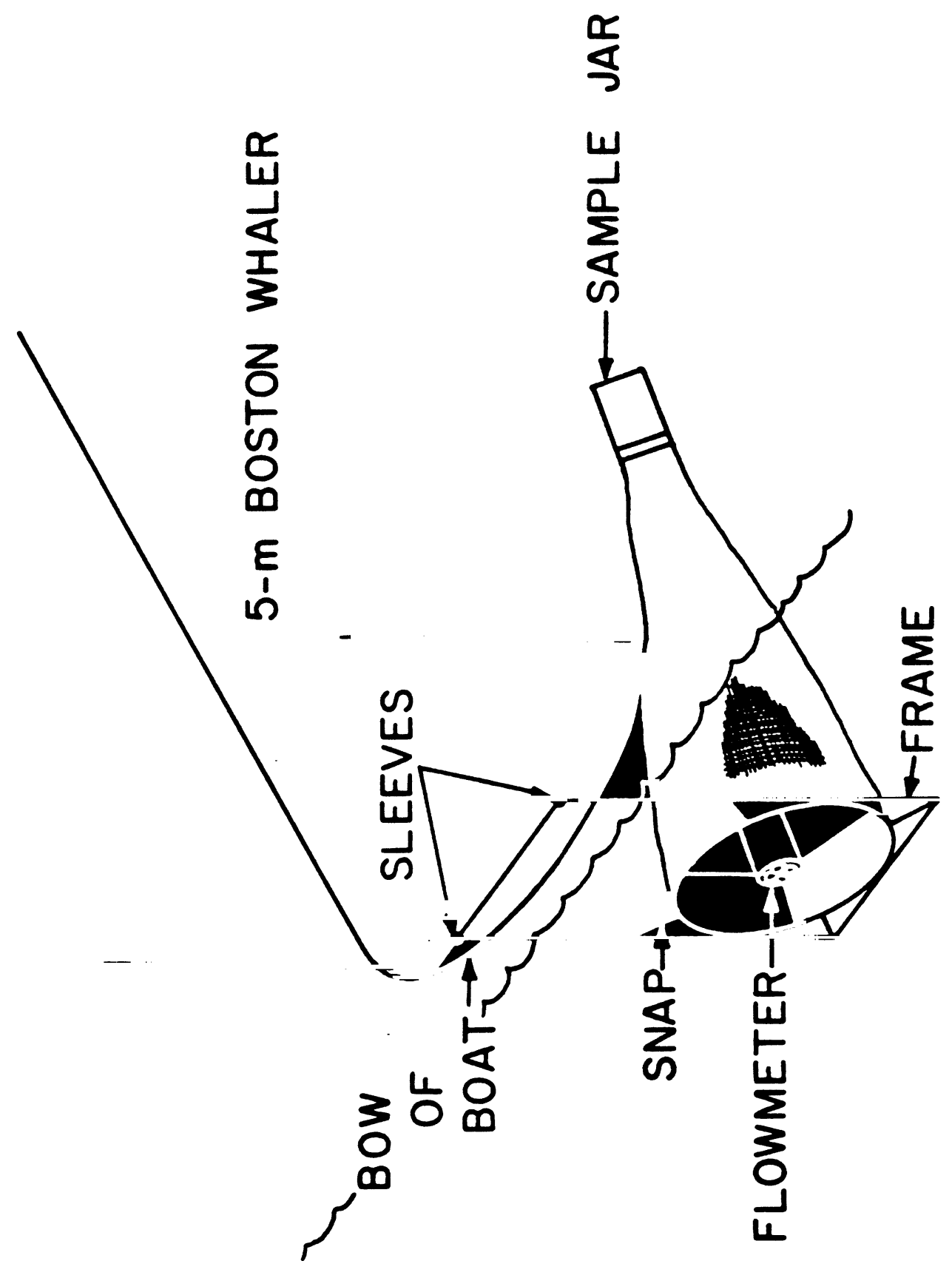

ع لِ

选

(

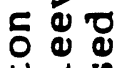

ثै न

装明

ธิ ข

드 है

崩 - n

屯面茴

高是

ซิ

E立

ס

잉

- 3

도잉

ข

E 3

至气

10

占

的莫

14

出造造

उ先起范

व

制

لَّل

วิ

멍

ro

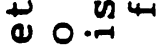

ᄃ車苋

둉

لِّد فِ

亗

له

임

-

ช

光 30

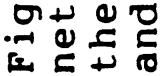




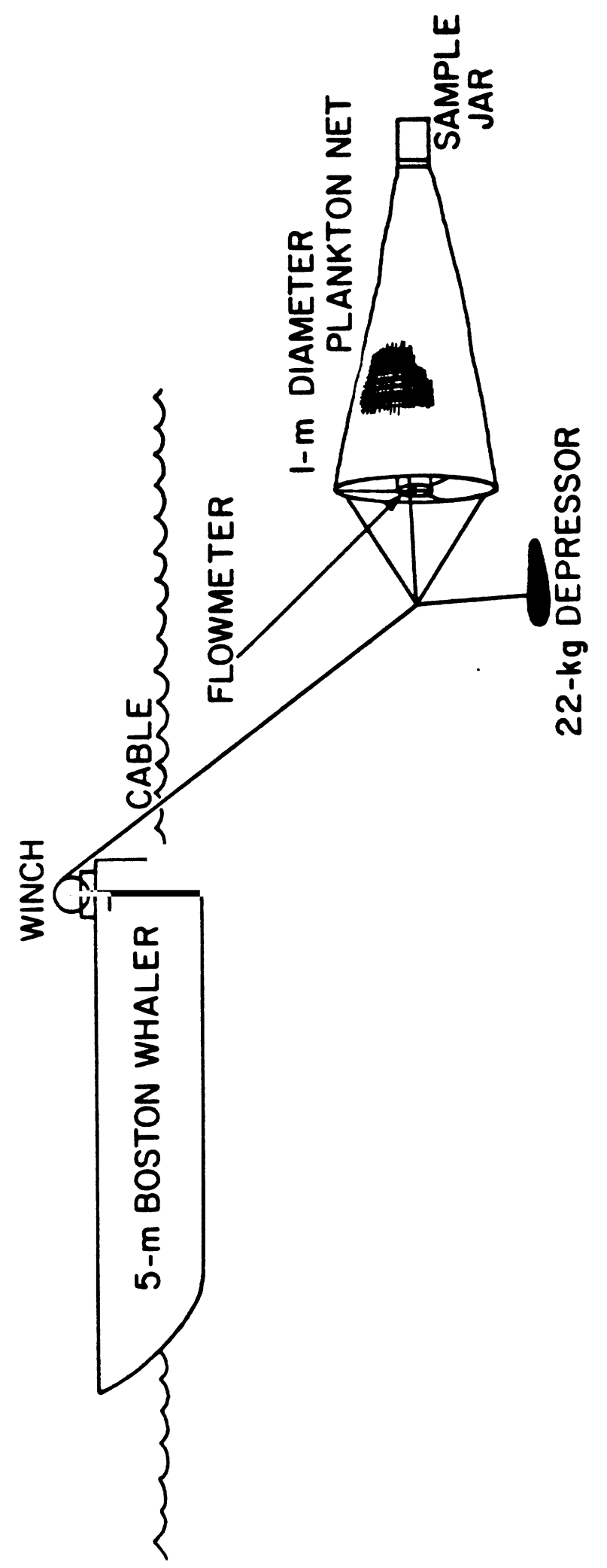

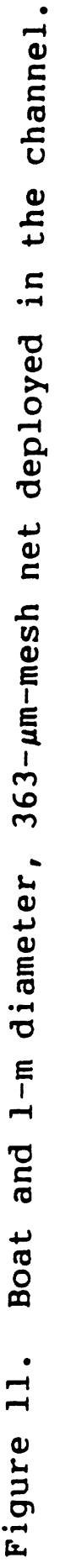


Sampling Schedule

Sampling was performed weekly ( 6 wk) from 26 April to 30 May 1985 (Table 3 shows sampling schedule). A total of 228 samples was collected, all at night.

\section{Sample Processing}

All $\mathrm{f}$ ish larvae samples were preserved immediately in $10 \%$ formaldehyde solution. Samples were sorted using binocular microscopes. Larval fish identifications were based on taxonomic descriptions by Auer (1982) and Cucin and Faber (1985). All fish larvae were measured to the nearest $0.1 \mathrm{~mm}$ (lake whitefish and lake herring) or $0.5 \mathrm{~mm}$ total length (larvae of other species) and enumerated. Rainbow smelt eggs, recognized by the presence of a stalk, were also counted.

Table 3. Dates when night larval fish sampling on the St. Marys River was conducted during 1985. a = shore $(\mathrm{m}), \mathrm{b}=2 \mathrm{~m}, \mathrm{c}=$ channel.

\begin{tabular}{ccc}
\hline Week No. & $\begin{array}{c}\text { Stations } \\
\text { Sampled }\end{array}$ & Dates \\
\hline 1 & 2,3 & 24 Apr \\
& $4-7$ & 26 Apr \\
& $5-7$ & $27-28$ Apr \\
2 & $2-4$ & $29-30$ Apr \\
& 1 & 30 Apr-1 May \\
\multirow{2}{*}{3} & 1 & 1 May \\
& $5-7$ & 6 May \\
4 & $2-4$ & $7-8$ May \\
& $2-4$ & $8-9$ May \\
& $5-7$ & $13-14$ May \\
5 & 1 & $14-15$ May \\
& 15 May \\
& $2-7$ & 22 May \\
6 & $2 c, 3,4$ & $23-24$ May \\
& $2 a, 25,5-7$ & 27 May \\
& & $28-29$ May \\
& & $29-30$ May \\
\hline
\end{tabular}




\section{RESULTS}

\section{MACROPHYTES}

\section{Introduction}

Studies were undertaken to describe the relationship between the degree of light penetration through the water column and maximum depth of occurrence of macrophytes. This was partly aimed at confirming the findings of Liston et al. (1986) and Liston and MCNabb (1986), who showed that in the clear waters of the upper reaches of the St. Marys River plants grow at greater depths than in the more turbid waters of the downstream portions.

\section{$\underline{\text { Results }}$}

The frequencies of plants occurring in grab samples taken along the $1-\mathrm{km}$ transects at the five sampling sites (Table 4) showed that members of the Characeae, a family of green algae, were the most abundant plants at all transects. In most samples the charophytes retrieved did not have reproductive structures, which are necessary for proper identification. Therefore, they are listed as Nitella sp. indeterminable or Chara sp. indeterminable. Most of the specimens of Nitella probably belonged either to the species Nitella flexilis or $N$. opaca. Only one species of Chara was identified, Chara fragilis, but one or two other species were probably also in the samples. In nearly all samples Nitella was present in much greater quantities than Chara.

Vascular piants were important at two sites, Izaak Walton Bay and Raber Bay. These were also the only two sites where all grab samples contained plants. In Raber Bay, samples were taken at depths which were probably $0.3-0.6 \mathrm{~m}$ shallower than the actual macrophyte bed boundary. This was the first site sampled and our subsequent experience showed that vascular macrophytes tended not to grow at depths as great as did the charophytes. Izaak Walton Bay is relatively shallow throughout $(\leq 5 \mathrm{~m})$ and no place was found where plants did not grow on the bottom; the grab-sample transect was located in the central deepest portion of the bay. Therefore, the data for Izaak Walton Bay are not from a true macrophyte boundary.

Fifteen sets of light readings were taken at each of the five river localities (see Appendixes 262-266). Each set of readings was used to calculate a vertical light extinction coefficient and all 15 were used to calculate a mean extinction coefficient for each site. A 95\% confidence interval for the mean extinction coefficient was derived using Student's t- 
Table 4. Plant frequency along $1-\mathrm{km}$ transects over the outer depth boundary of submerged macrophyte beds in $f$ ive locations in the St. Marys River, August 1985. Frequencies are given in terms of the percentage of grab-samples in which the plant occurred.

$\overline{-}$

Plants

Nitella sp. indeterm.

Nitella flexilis

Chara sp. indeterminable

Chara globularis

Potamogeton sp. indeterm.

Potamogeton praelongus

Potamogeton gramineus

Potamogeton Iichardsonil

Elodea canadensis

Vallisneria americana

Isoëtes macrospora

No plants in sample

Sampling depth (m)

Number of samples
Izaak

Walton Lake West Lake East Lake Raber Bay Nicolet Munuscong Munuscong Bay

33.

86.7

70.0

53.1

77.4

56.7

6.7

9.425 .8

50.0

6.7

36.7

18.8

71.0

20.0

3.2

6.7

13.3

3.2

23.3

3.2

3.2

3.3

6.7

10.0

0

13.3

13.3

34.4

0

4.5

8.2

5.2

4.9

4.0

30

30

30

32

31 
statistic (Mendenhall 1971). The mean extinction coefficients, with their confidence intervals, are plotted against the depth of the macrophyte boundary (Fig. 12). Also plotted are two regression Iines; the data from Izaak Walton Bay were omitted from the regression calculations because they do not correspond to the true maximum depth of macrophyte occurrence. The extinction coefficients from the 15 sets of light readings from each of the four other sites were used in calculating $I$ ine $A$, and line $B$ was fitted to the mean extinction coefficients from the four sites. Line B shows a much stronger relationship between macrophyte boundary depth and light extinction coefficient ( $r=-$ $0.976)$ than line $A(r=-0.655)$. All correlation coefficients cited are significantly different from zero, $p<0.02$. This is due to the great variability in the light extinction measurements, particularly those from the three downstream stations.

In their investigations, Liston et al. (1986) found macrophytes growing on the bottom of the navigation channel north of Izaak Walton Bay, at depths greater than $10 \mathrm{~m}$. If we assume that the depth of the macrophyte boundary would lie at $10.5 \mathrm{~m}$ in the bay, and use the light extinction coefficient data from that site and the remaining four in regression calculations, we obtain the results shown in Figure 13. Curve $C$, obtained by polynomial regression of all 75 extinction coefficient measurements from the five sites, shows a moderately strong relationship between depth and extinction coefficients $(r=-0.897)$. An even stronger correlation $(r=-0.968)$ is shown by line $D$, which is derived by linear regression of the five mean light extinction coefficients.

\section{DRIFT: BENTHOS, FISH LARVAE, FISH EGGS, AND MACROPHYTES}

\section{Introduction}

Benthic drift collected by coarse- (355 $\mu \mathrm{m})$ and fine- (153 $\mu \mathrm{m})$ mesh nets during winter (ice-cover conditions) and summer (ice-free conditions) in the St. Marys River was represented by 71 benthic taxa, 5 larval $f$ ish taxa, and 12 macrophytic taxa (Table 5). Due to study design, all stations at each transect were sampled by coarse-mesh nets only; therefore, results presented here will consider only those estimates derived from coarse-mesh nets unless otherwise stated.

\section{Mesh-size Comparisons}

In nearly all comparisons, total benthic and larval fish drift densities estimated by fine-mesh nets exceeded that of concurrently set coarse-mesh nets. Only in about half of these comparisons was the difference statistically significant, with total benthic and larval fish densities in fine-mesh nets always 


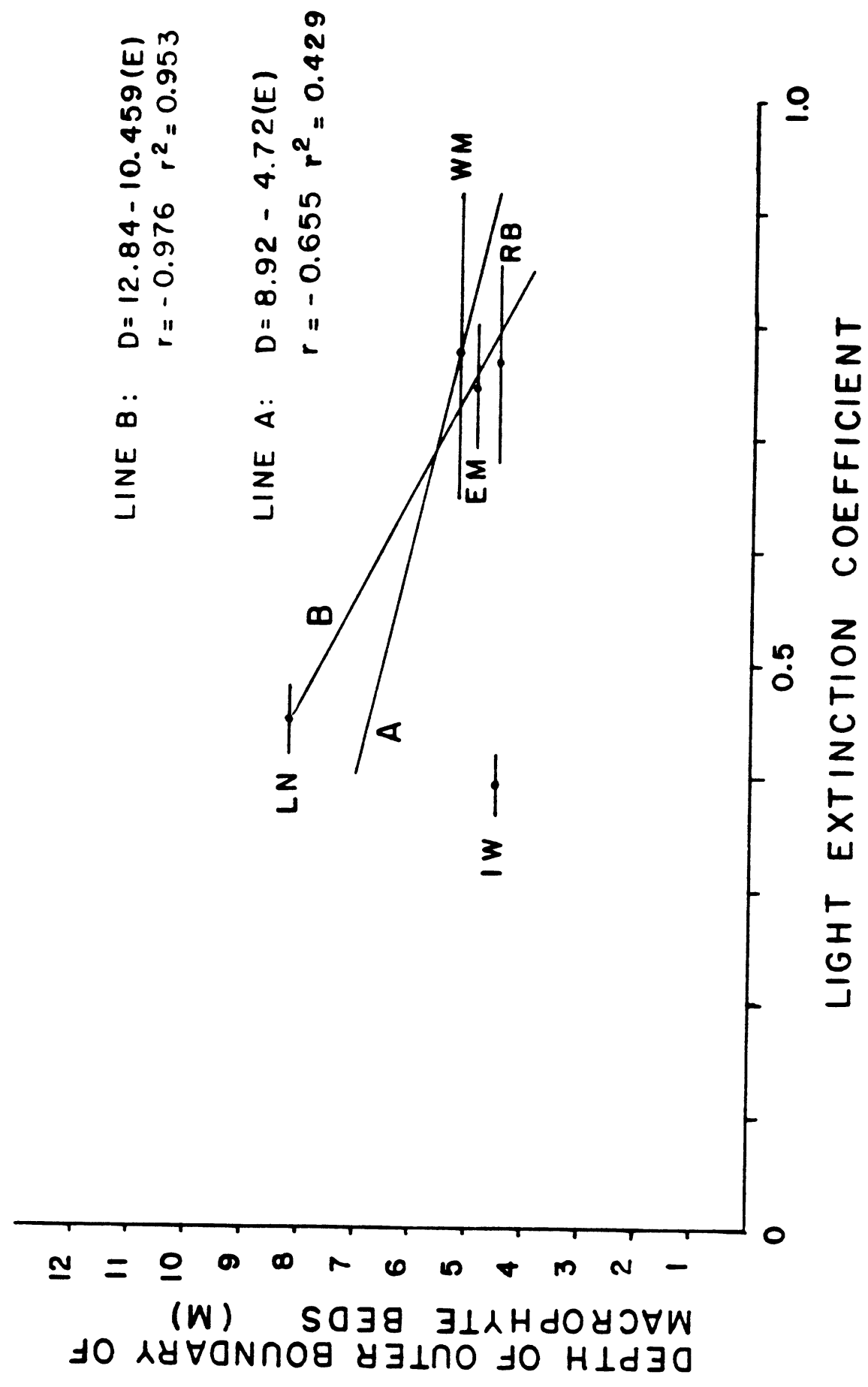

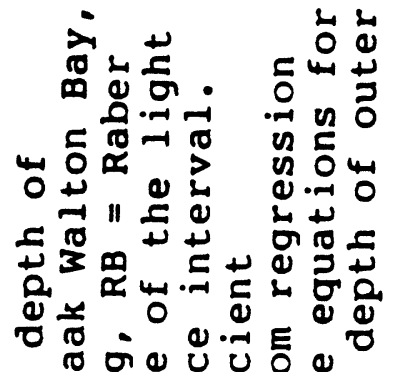

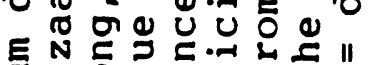

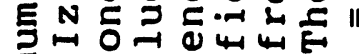

.

$x$ ไู

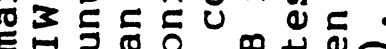

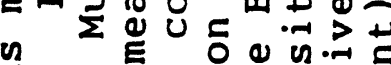

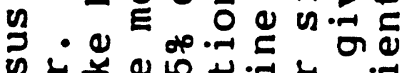

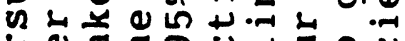

เै

$>$. n... $n-4$

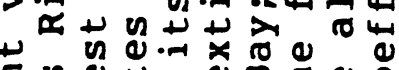

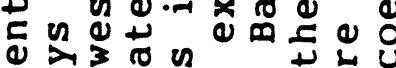

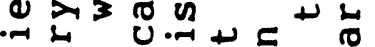

U U

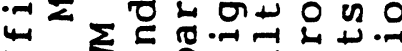

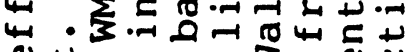

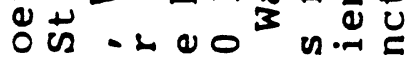

U ตัก๊

동

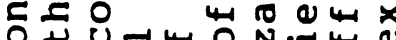

$\therefore$ प

U.

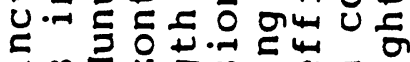

- n

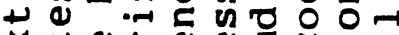

$x+0$ \& ब

c.)

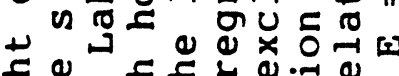

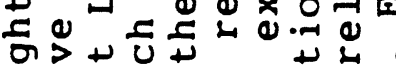

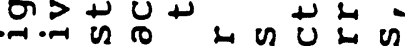
न世 的 तै กิ

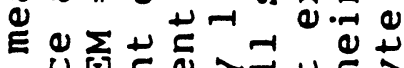

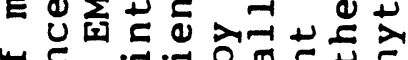

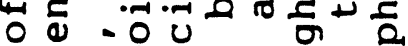
प

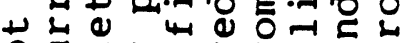

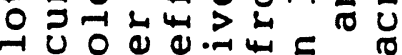

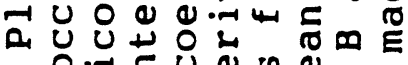

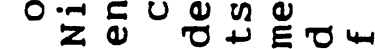

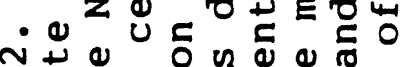

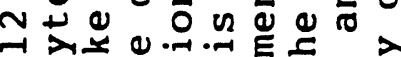

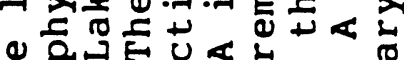
t วั

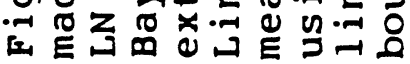




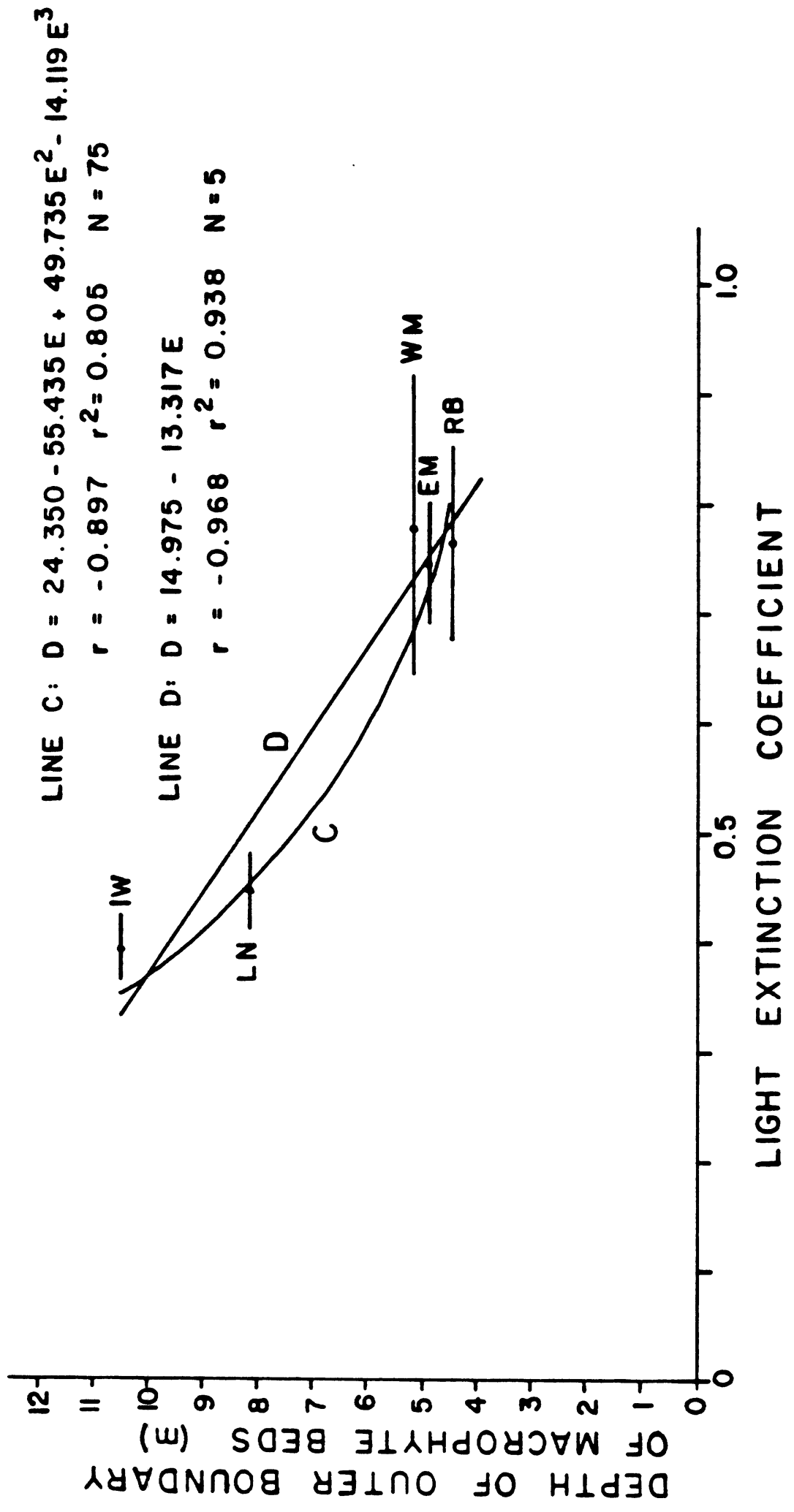

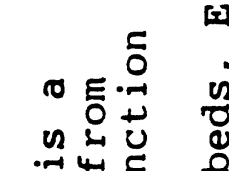

Un⿺辶万

ه

त

글으.

प्र

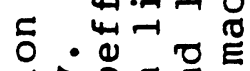

- $>$ 岂口它

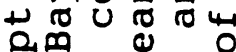

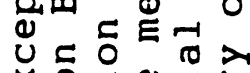

$\times 0 .-10$.

$0+4$ 능

(1)

도네

누응

듀

$\because N+N$ N

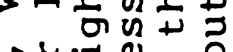

त म्न

N 0 -

त4

రृ

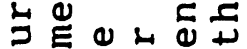

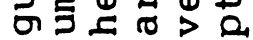

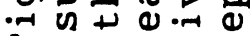

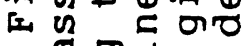

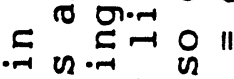

•- U य ग

ข

ه

+ - $>>$ in

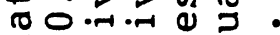

ठ대

ข

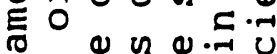

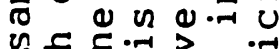

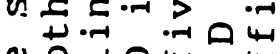

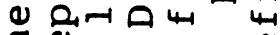

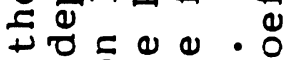

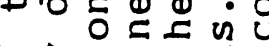

4 >.

○ น U

廿 0 0

O

ฉ.

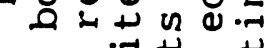
- 0 - U $m+\infty \quad 00$

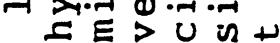

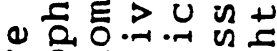
$\Rightarrow 05440$ व כ ᄂ $>$ w w. бण

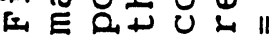




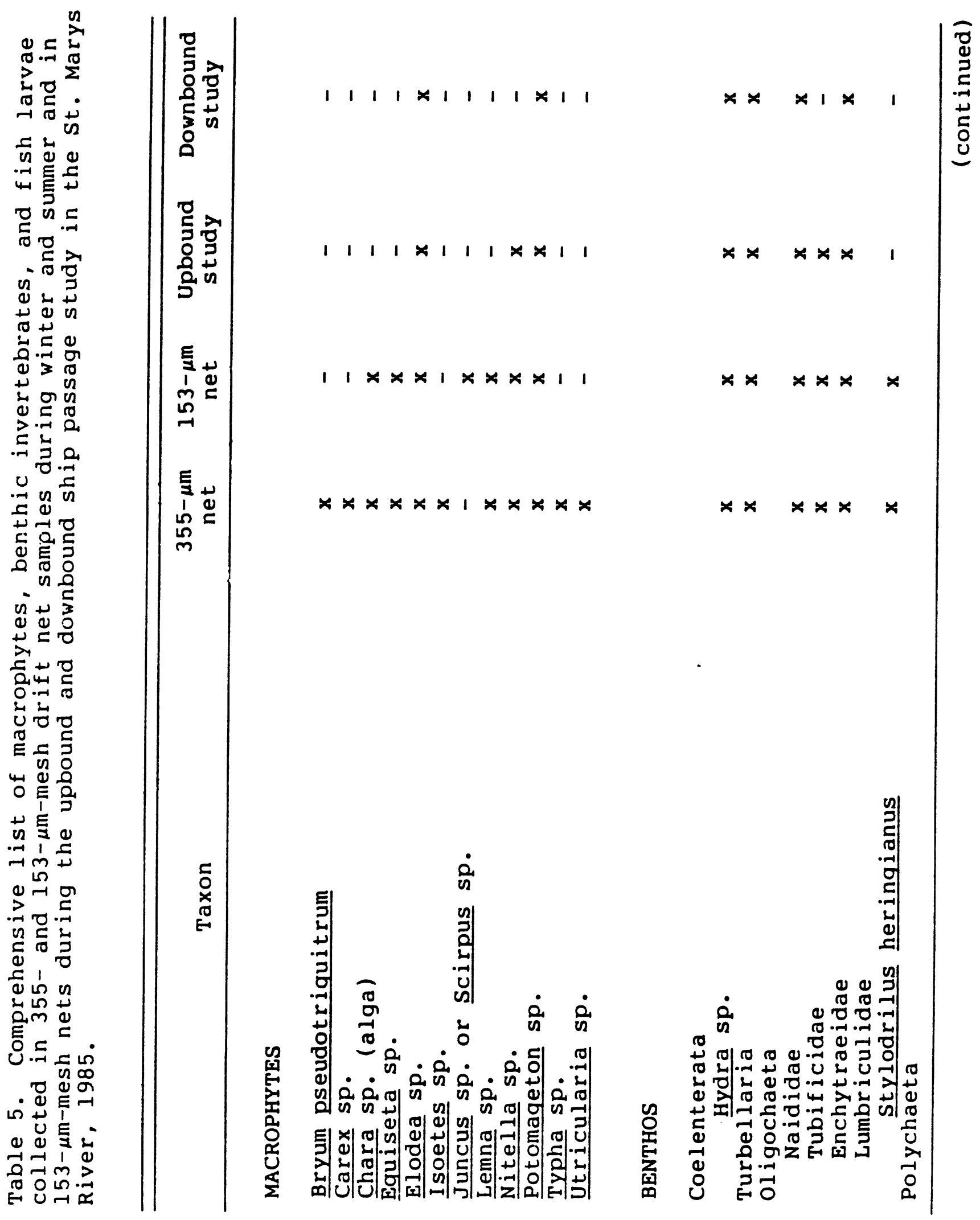




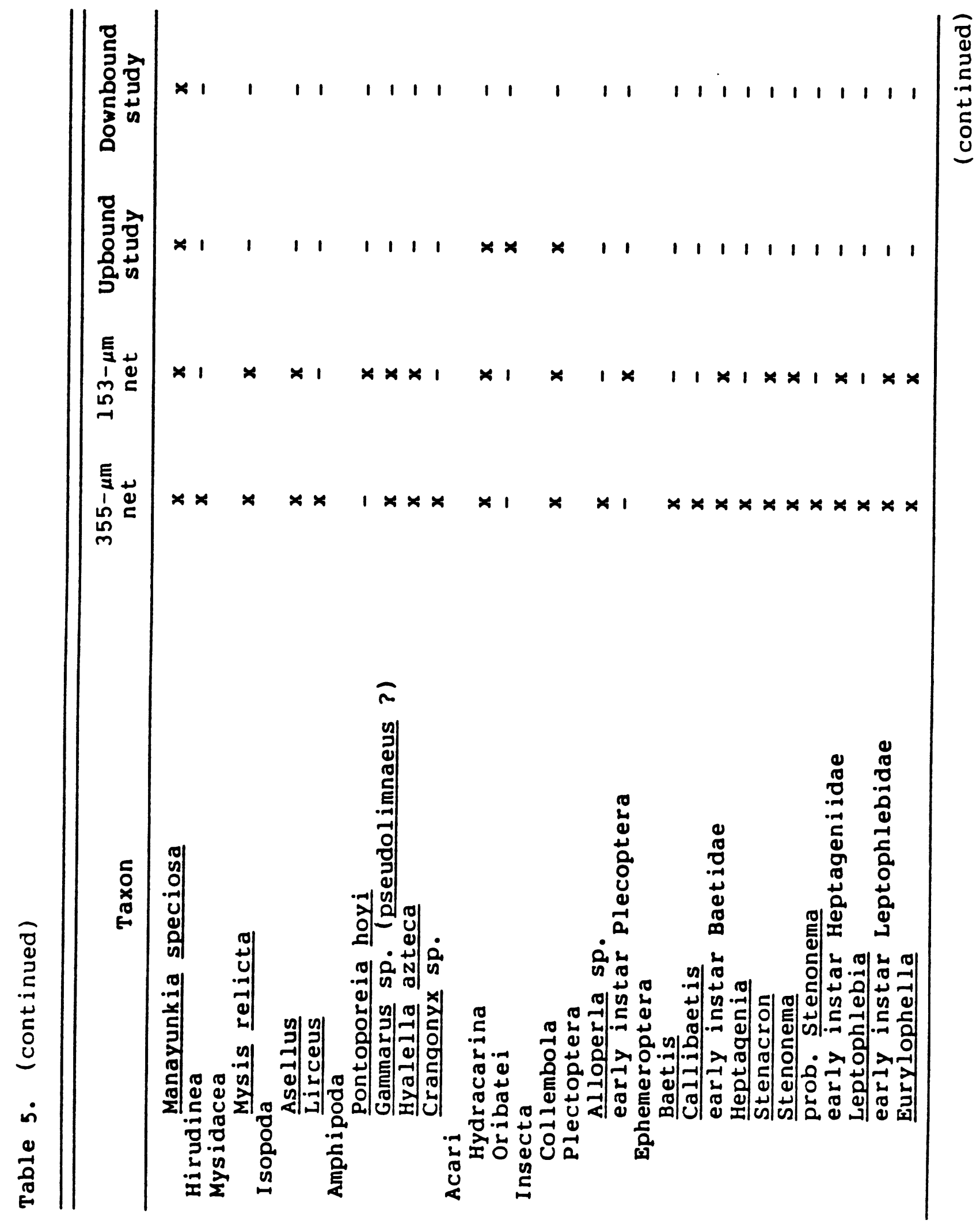




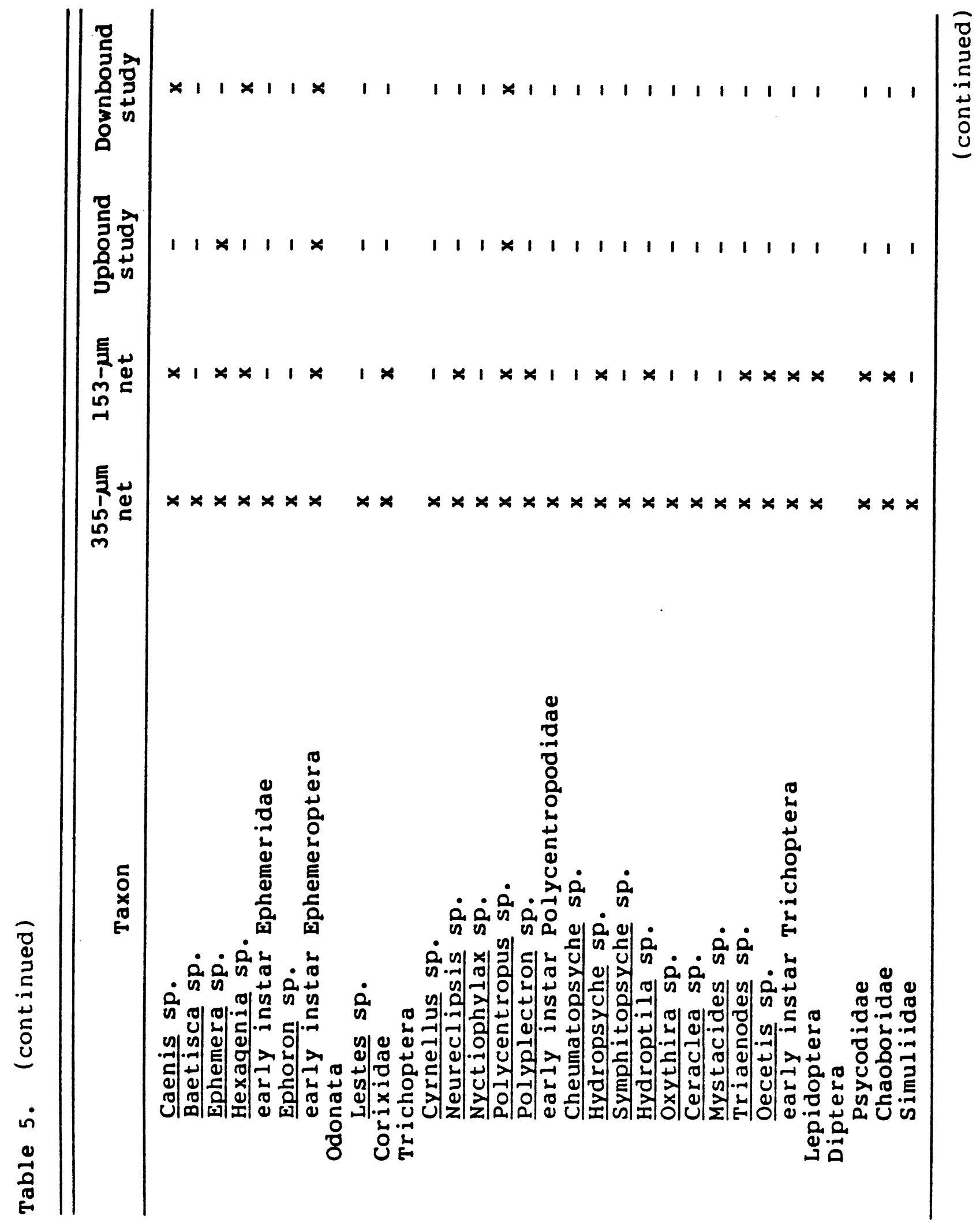




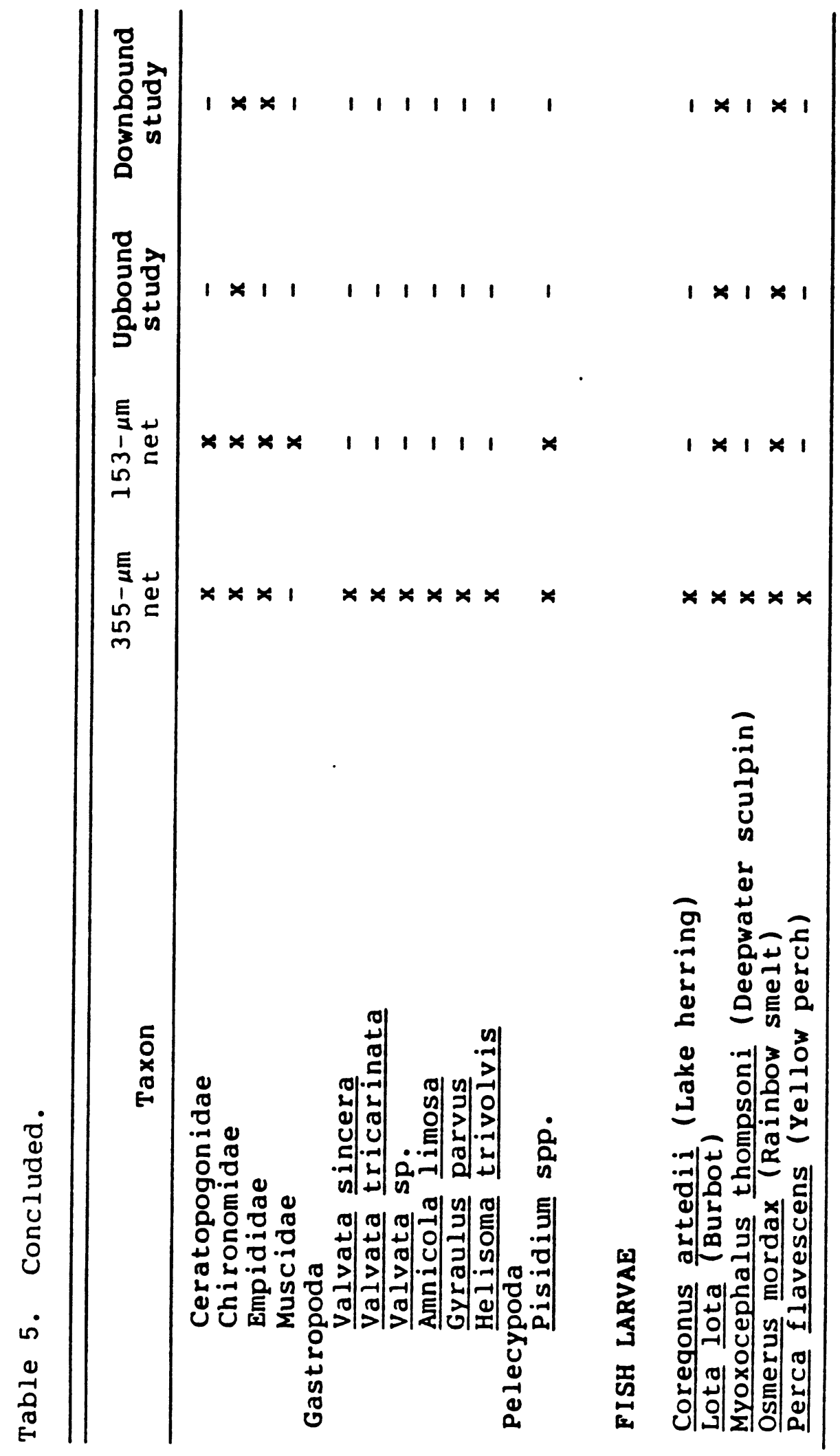


exceeding coarse-mesh net densities. At Frechette Point, net comparisons for stations 3-5 indicated the difference in total benthic drift density was significant only at station 5 in winter and only at station 4 during summer. When averaged over all stations, there was no significant difference in total benthic drift density between mesh sizes during winter. However, during summer, total benthic drift density averaged over all stations was significantly greater in fine- $\left(1,562 / 1,000 \mathrm{~m}^{3}\right)$ than coarse- $\left(1,153 / 1,000 \mathrm{~m}^{3}\right)$ mesh nets (Tables 6 and 7 ).

At stations 3 and 4 , summer larval fish drift density was significantly greater in fine-mesh nets $\left(129 / 1,000 \mathrm{~m}^{3}\right.$ and $233 /$ $1,000 \mathrm{~m}^{3}$, respectively) when compared with coarse-mesh nets (59/ $1,000 \mathrm{~m}^{3}$ and $77 / 1,000 \mathrm{~m}^{3}$, respectively). There were no meshsize-related density differences at station 5 . When averaged over all samples at stations 3-5, fine-mesh nets retained significantly greater numbers of $f$ ish larvae than did coarse-mesh nets $\left(183 / 1,000 \mathrm{~m}^{3}\right.$ vs. $\left.87 / 1,000 \mathrm{~m}^{3}\right)$.

At Lake Nicolet, fine-mesh net estimates of total benthic drift density were significantly greater only at stations 6 and 7 during winter; whereas, in summer this was the case only at station 4. However, when averaged over all stations, fine-net estimates of total benthic drift density exceeded those of coarse nets during both winter $\left(102 / 1,000 \mathrm{~m}^{3}\right.$ vs. $\left.18 / 1,000 \mathrm{~m}^{3}\right)$ and summer $\left(1,383 / 1,000 \mathrm{~m}^{3}\right.$ vs. $\left.670 / 1,000 \mathrm{~m}^{3}\right)($ Tables 6 and 7$)$.

Mesh-size-related differences in drifting fish larvae densities were significant only at navigation channel stations 4 and 6 in Lake Nicolet. At station 4 , the number of fish larvae retained by $f$ ine-mesh nets $\left(1,972 / 1,000 \mathrm{~m}^{3}\right)$ was significantly greater than that retained by coarse-mesh nets $\left(661 / 1,000 \mathrm{~m}^{3}\right)$. Similarly, in the upbound channel, the fine-mesh nets $(778 / 1,000$ $\mathrm{m}^{3}$ ) had a significantly greater number of $\mathrm{fish}$ larvae than did coarse-mesh nets $\left(323 / 1,000 \mathrm{~m}^{3}\right)$. When averaged over all samples at stations $3,4,6$, and 7 having concurrently set fine- and coarse-mesh nets, larval $f$ ish drift density was significantly greater in fine-mesh nets $\left(1095 / 1,000 \mathrm{~m}^{3}\right)$ than coarse-mesh nets $\left(501 / 1,000 \mathrm{~m}^{3}\right)$.

At Point aux Frenes, the difference between total benthic drift density catches based on mesh size was significant at all stations except station 3 during both seasons. When averaged over all stations, estimates of total benthic drift density were significantly greater in $f$ ine when compared with coarse-mesh nets during both winter $\left(150 / 1,000 \mathrm{~m}^{3}\right.$ vs. $\left.25 / 1,000 \mathrm{~m}^{3}\right)$ and summer $\left(764 / 1,000 \mathrm{~m}^{3}\right.$ vs. $\left.183 / 1,000 \mathrm{~m}^{3}\right)$ (Tables 6 and 7$)$.

Ratios of fine to coarse-mesh net densities suggested a variety of relative catch efficiencies dependent upon taxon, season, and transect (Tables 6 and 7 ). However, when comparisons 


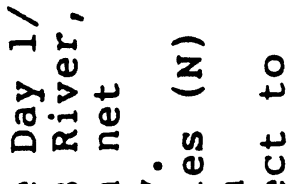

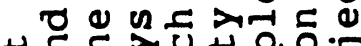

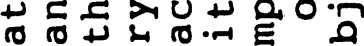

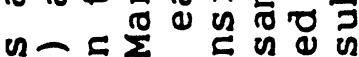

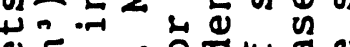
Q E • O = थ世

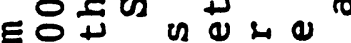

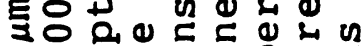

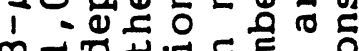

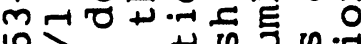

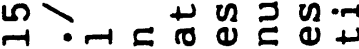
O-.川PE 0 ○

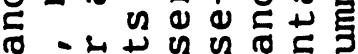
ه E $>0$ \%

300 은

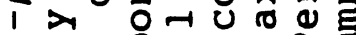

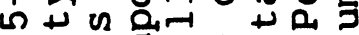

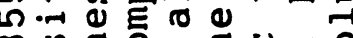

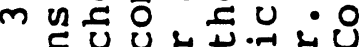
ᄃ U U म म

-

Uफ 0 으르.

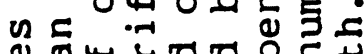
(1)

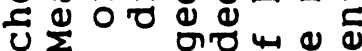
$+{ }^{2}$ o $u$ o (1) E. \& in $>$

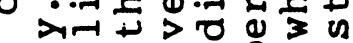

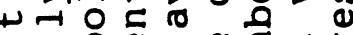

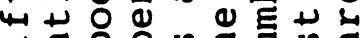

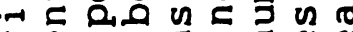
4 110 .

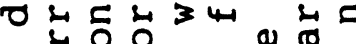

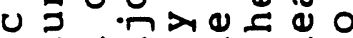
- U U ס ट $ᄃ 0$ E.

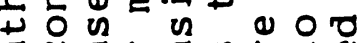

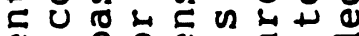
a 2 Q 0 \%

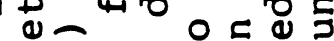
4 U E $20 \cdot \omega$ ह

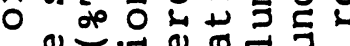

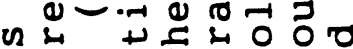
Cथ थ

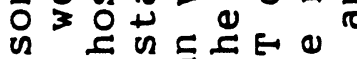

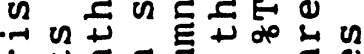
$4+2$ 둥

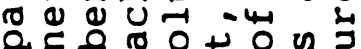

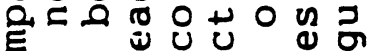

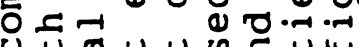
年

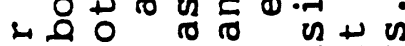

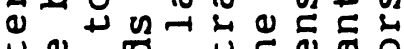

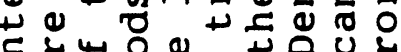

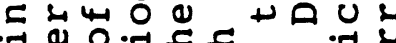
$320+0$ U 4 r 0 ro

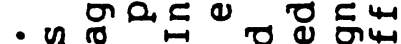

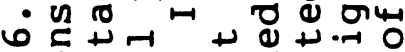
0 ह U.न U

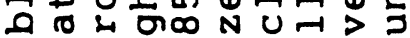
E थ

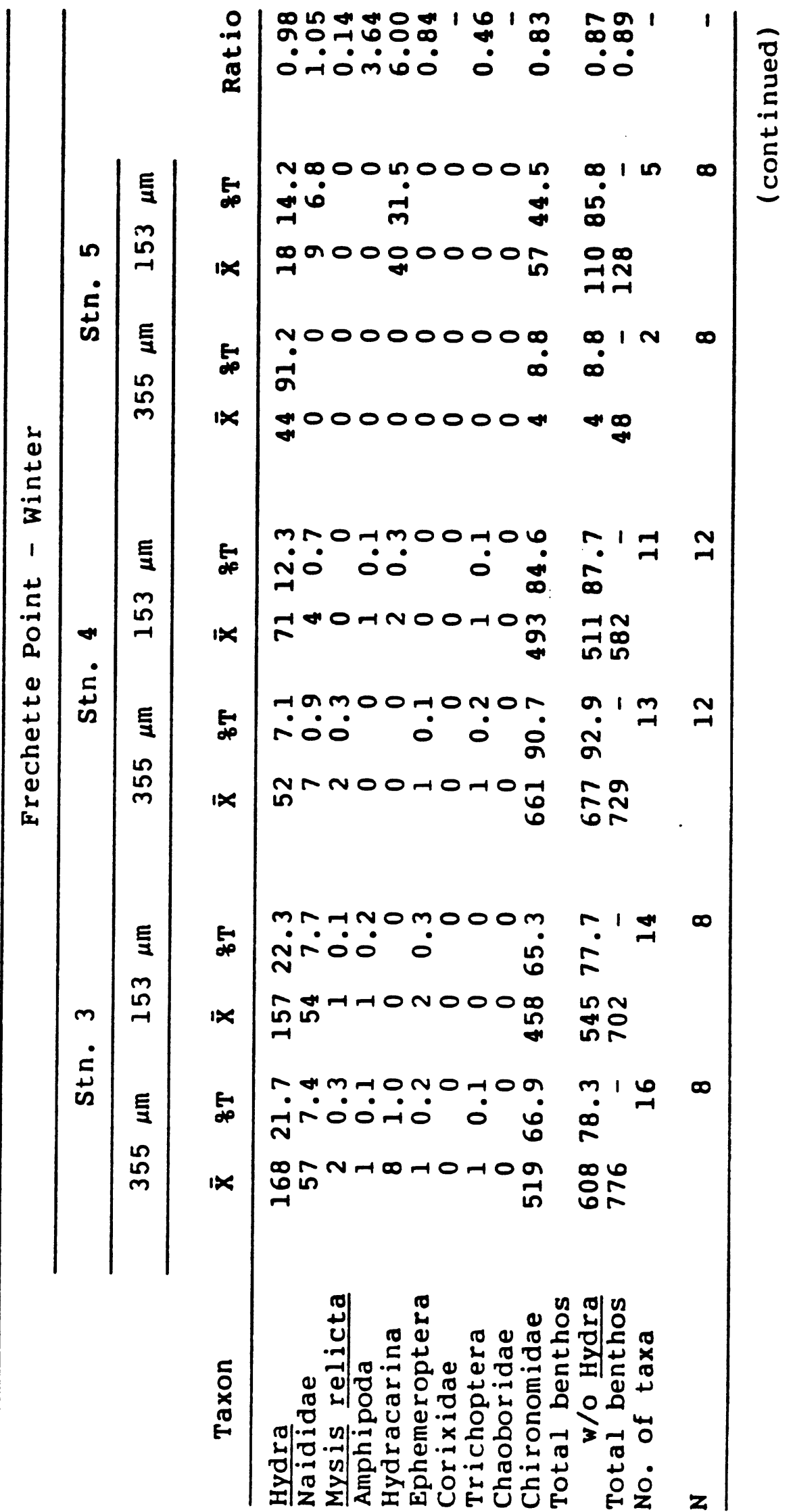




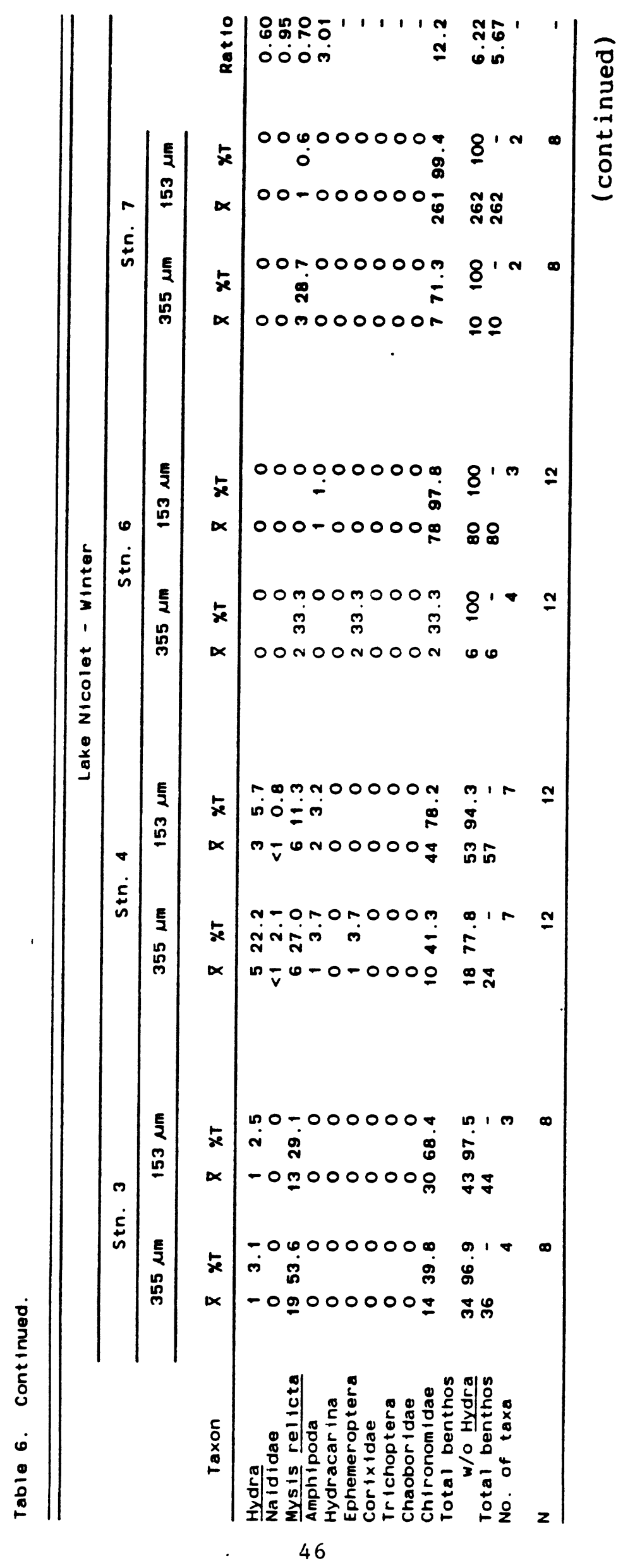




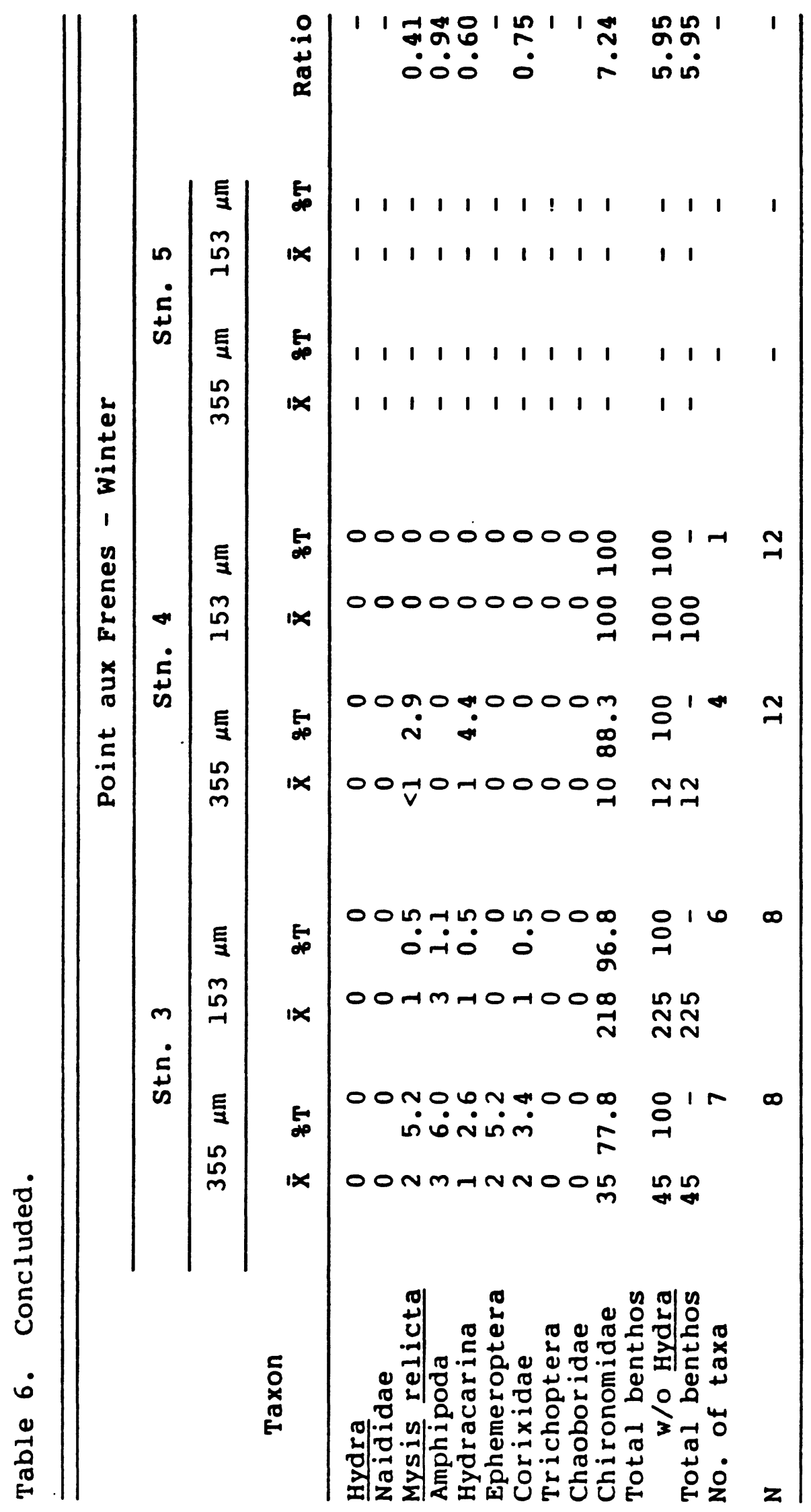




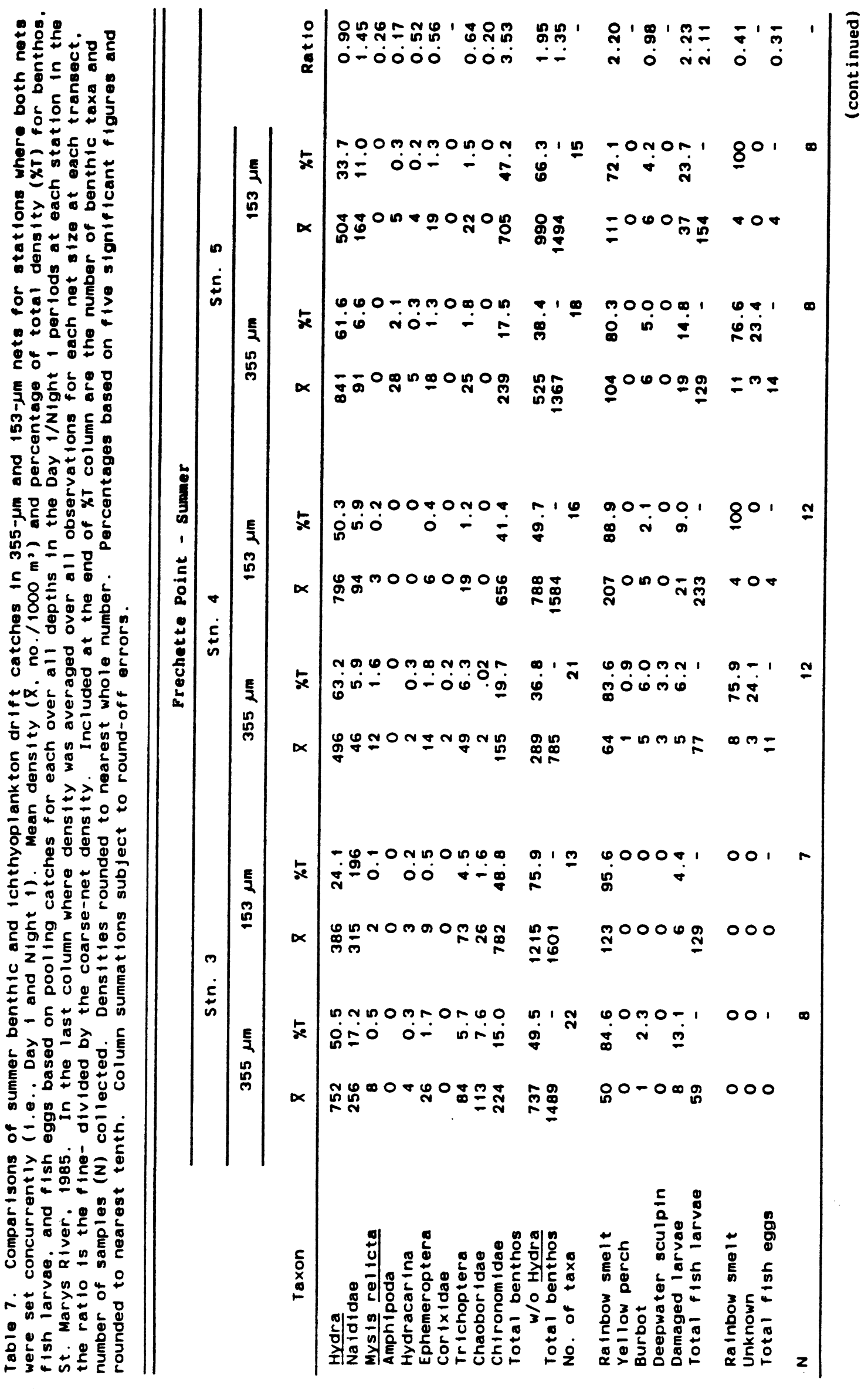




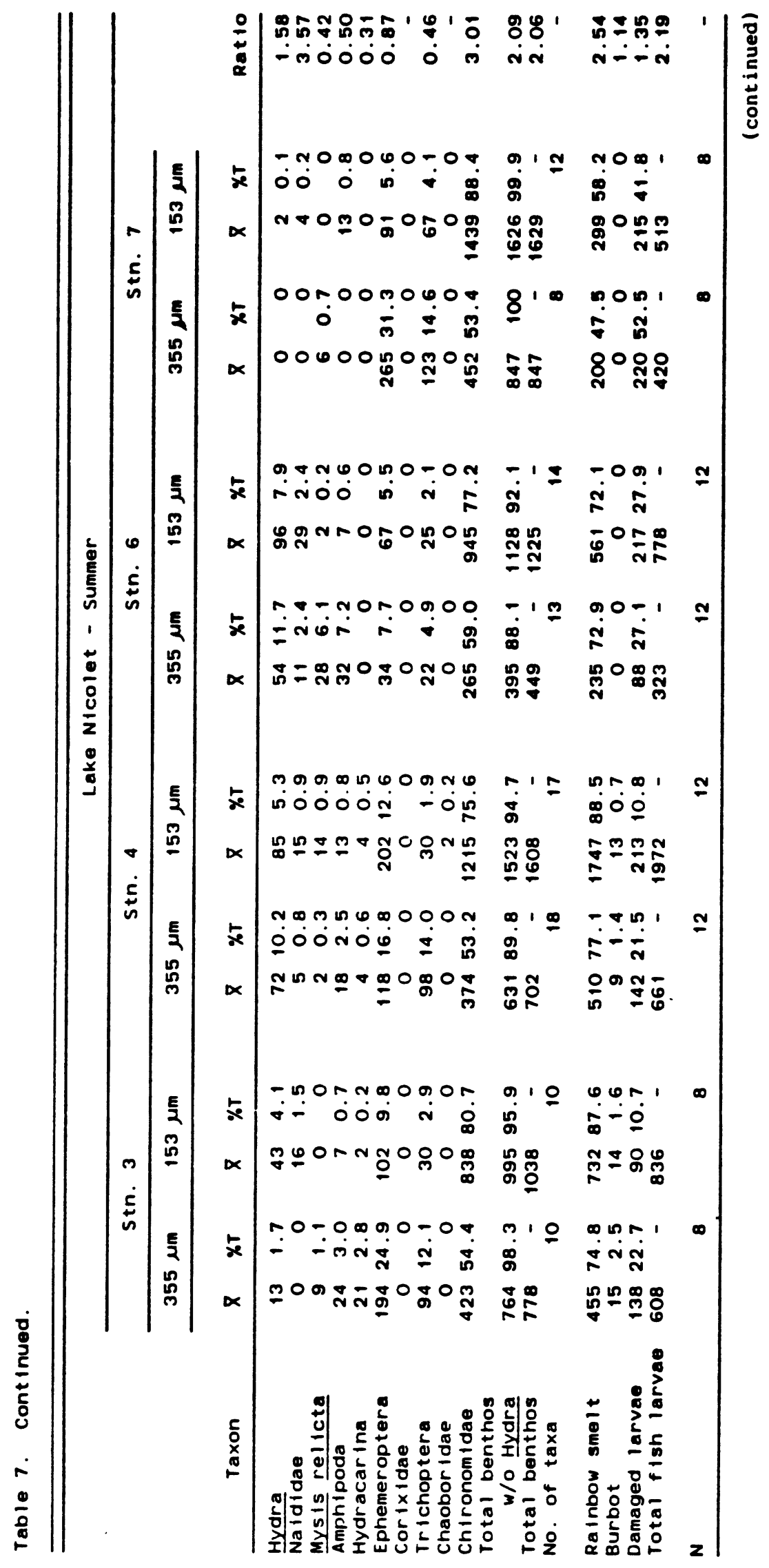




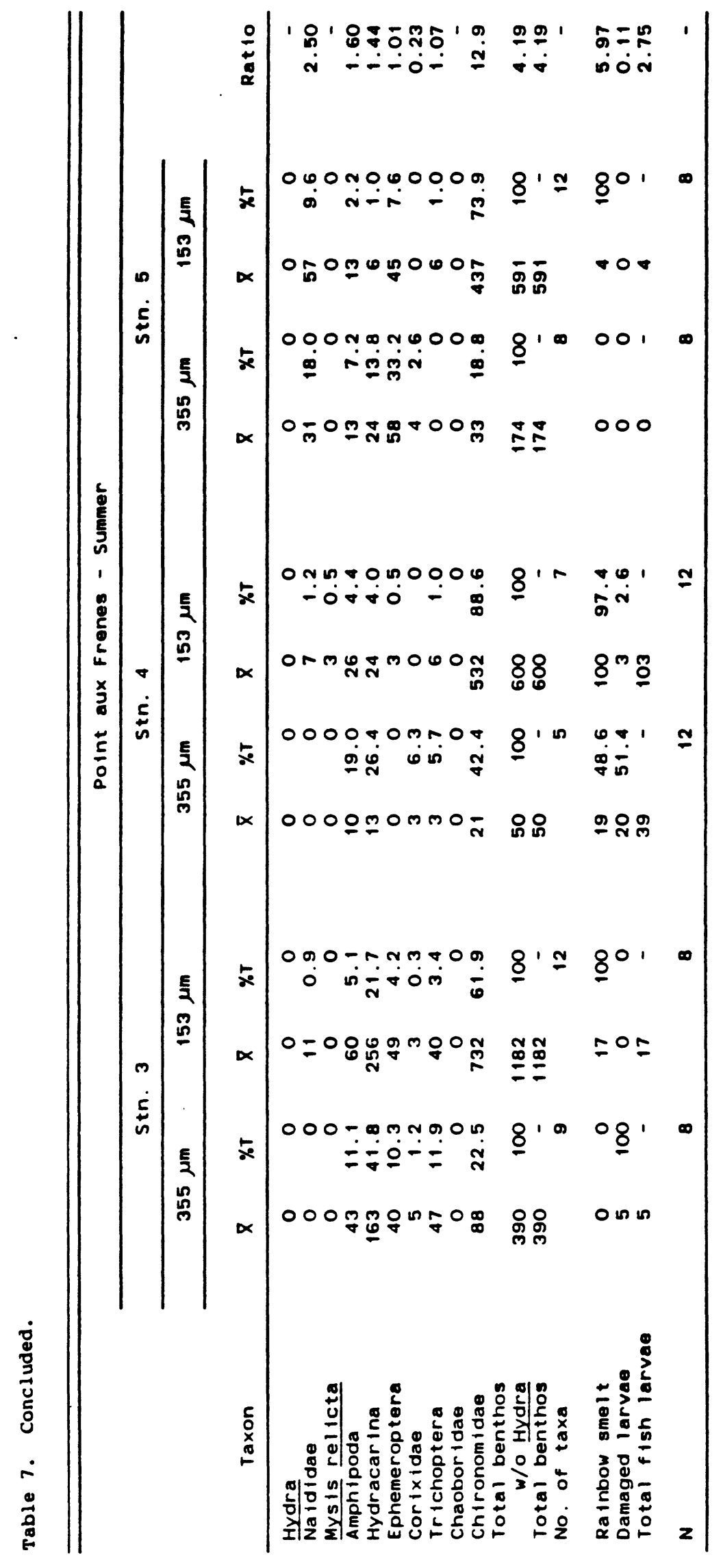


of $\log$ densities for each major component of the drifting benthos were made for densities averaged over all coarse and $f$ ine-mesh nets, respectively, significant density differences were noted only for Naididae, Chironomidae, total benthos without Hydra, total benthos, and Mysis relicta. In all but the latter comparison, $i . e .$, for $M$. relicta, densities in $f$ ine-mesh nets significantly exceeded those in coarse-mesh nets by a ratio ranging from 1.52 to 2.80 . For $M$. Ielicta, the coarse-net density estimate $\left(5.5 / 1,000 \mathrm{~m}^{3}\right)$ was greater than that of the fine-mesh net $\left(2.7 / 1,000 \mathrm{~m}^{3}\right)$ by a ratio of 2.06 .

At Point aux Frenes, there were no significant larval fish drift density differences among mesh sizes at stations 3, 4, and 5 or at stations 3-5 combined. However, in all comparisons, densities in fine-mesh nets exceeded those in coarse-mesh nets.

At stations and times where nets of both sizes were set concurrently, 59 benthic taxa were collected. While the number of taxa retained by coarse nets ( 50 taxa) was greater than that of fine nets ( 40 taxa), this trend was not generally evident. Within each station pooled over all depths at each transect and during each season, differences in the number of taxa retained by each mesh size never exceeded three. Among those comparisons where the differences were at least three, the number of taxa in $f$ ine-mesh nets exceeded those in coarse-mesh nets. Only at Frechette Point during summer were there any substantive differences in the number of taxa retained by the two mesh sizes. At Frechette Point, coarse-mesh nets consistently collected three to nine more taxa than did $f$ ine-mesh nets.

When comparing percent occurrence of macrophytes for the fine- and coarse-mesh sizes over all transects and stations, percent occurrence in $f$ ine-mesh nets was greater in winter (61\%) and summer $(64 \%)$ than in coarse-mesh nets $(39 \%$ and $48 \%$, respectively). However, there was no difference in dominant plant taxa for coarse- and $f$ ine-mesh nets between seasons or for winter and summer seasons between mesh sizes. In all cases except fine-mesh nets in summer, the dominant macrophytes were Nitella, Potamogeton, and Elodea in order of decreasing percent occurrence. Among fine-mesh nets in the summer, the order of the last two taxa was reversed.

Seasonal and Transect Comparisons of Drift Densities

General--

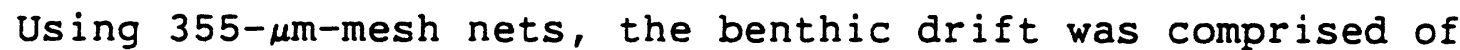
67 taxa which averaged $562 / 1,000 \mathrm{~m}^{3}$ when pooled over all samples collected (Table 8 ). The dominant forms were Chironomidae (183/ $\left.1,000 \mathrm{~m}^{3}\right)$, Hydra $\left(170 / 1,000 \mathrm{~m}^{3}\right)$, and Ephemeroptera $\left(73 / 1,000 \mathrm{~m}^{3}\right)$. 


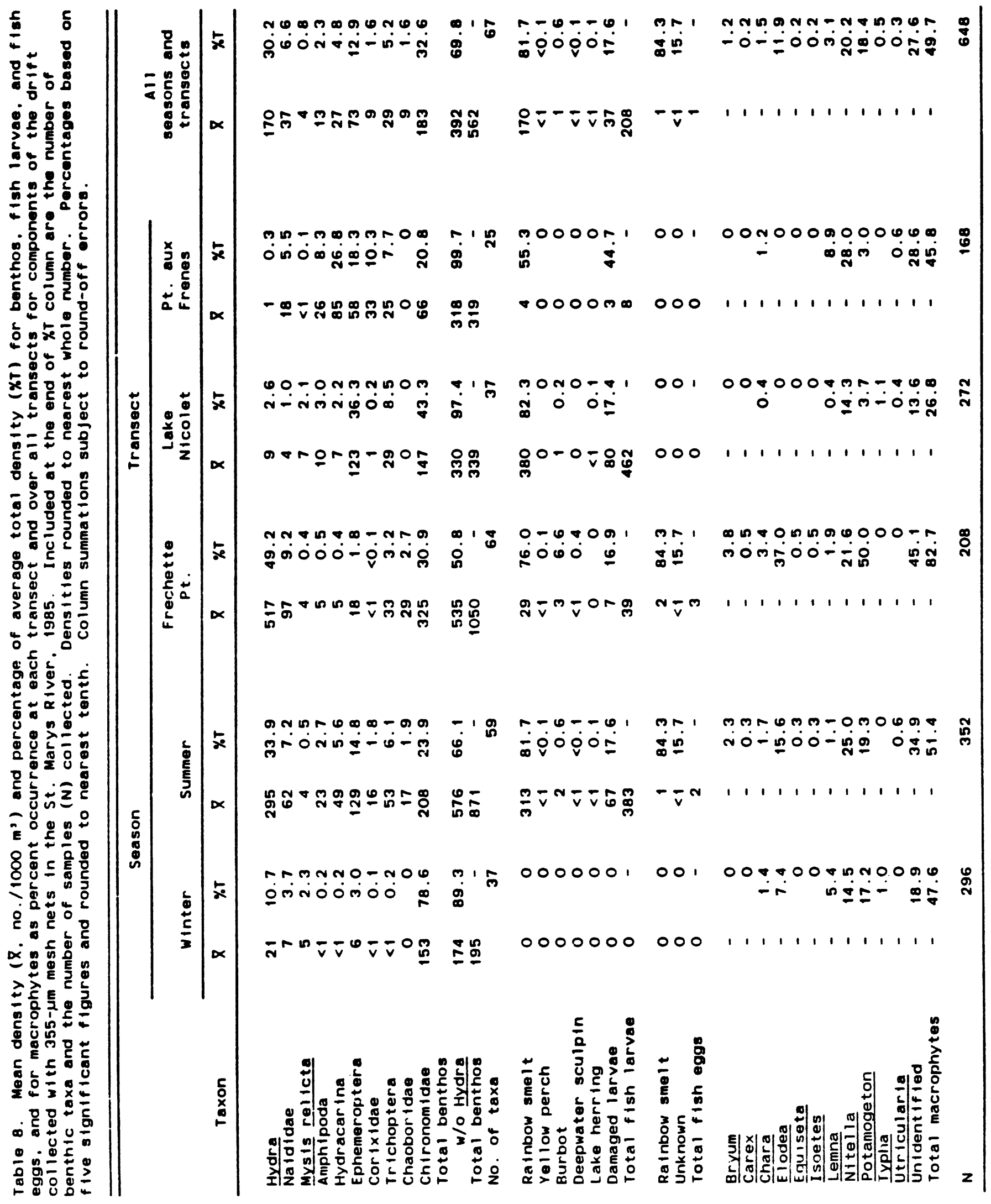


Seasonally, both the number of taxa and average benthic drift estimates were lower in the winter ( 37 taxa and $\left.195 / 1,000 \mathrm{~m}^{3}\right)$ than during the summer (59 taxa and $871 / 1,000 \mathrm{~m}^{3}$ ) period. Although no fish larvae or eggs were caught during the winter, fish larval fish species were collected during the summer. Average total larval $f$ ish drift was $383 / 1,000 \mathrm{~m}^{3}$. The number of $f$ ish eggs retained by coarse-mesh nets in the summer averaged $2 /$ $1,000 \mathrm{~m}^{3}$ (Table 8).

\section{Benthos--}

Average winter total benthic drift density was significantly greater at Frechette Point when compared with those at Lake Nicolet or Point aux Frenes, which were not significantly different. Benthic drift pooled over all winter samples at Frechette Point averaged $482 / 1,000 \mathrm{~m}^{3}$, while at Lake $\mathrm{Nicolet}$ and Point aux Frenes similar averages were $49 / 1,000 \mathrm{~m}^{3}$ and $21 / 1,000$ $\mathrm{m}^{3}$, respectively (Table 9; Appendixes 8-27, 48-73, 102-114). Additionally, a greater number of taxa was collected at Frechette Point (36 taxa using both mesh sizes; $355 \mu \mathrm{m}=34,153 \mu \mathrm{m}=17$ ) than at Lake Nicolet ( 14 taxa using both mesh sizes; $355 \mu \mathrm{m}=12$, $153 \mu \mathrm{m}=8$ ) or Point aux Frenes ( 10 taxa using both mesh sizes; $355 \mu \mathrm{m}=9,153 \mu \mathrm{m}=6)$. Chironomidae was the dominant taxon at all transects. At Frechette Point Chironomidae made up $81 \%$ of average benthic drift density; at Lake Nicolet it was $52 \%$, and at Point aux Frenes it was 82\%. Remaining components of the drift community structure varied at each transect. At Frechette Point, Hydra was the second-most dominant taxon making up $12 \%$ of benthic drift followed by Naididae (4\%). At Lake Nicolet, Ephemeroptera (largely Leptophlebia) was the second-most dominant (24\%) followed by Mysis relicta $\left(17 \%, 8 / 1,000 \mathrm{~m}^{3}\right)$. At Point aux Frenes, Corixidae (6\%), Amphipoda (5\%, largely Hyalella azteca), and Mysis relicta (3\%) were the only remaining taxa, other than Chironomidae, making up a significant portion of benthic drift.

As during winter, average summer total benthic drift density at Frechette Point $\left(1614 / 1,000 \mathrm{~m}^{3}\right)$ was significantly greater than that at Lake Nicolet $\left(597 / 1,000 \mathrm{~m}^{3}\right)$ or Point aux Frenes (502/ $1,000 \mathrm{~m}^{3}$ ) (Table 9; Appendixes 28-47, 74-101, 115-134), which were not significantly different. The total number of taxa collected decreased from 55 taxa at Frechette Point to 35 taxa at Lake Nicolet and 22 taxa at Point aux Frenes. Chironomidae had the highest density of taxa at Lake Nicolet where it averaged $252 / 1,000 \mathrm{~m}^{3}$ (42\% of benthic drift). Ephemeroptera at Lake $\mathrm{Nicolet}$ averaged $222 / 1,000 \mathrm{~m}^{3}$ [largely Hexagenia $\left(155 / 1,000 \mathrm{~m}^{3}\right)$ and Caenis $\left.\left(53 / 1,000 \mathrm{~m}^{3}\right)\right]$ and made up $37 \%$ of summer benthic drift. At Frechette Point, Chironomidae drift density decreased to $258 / 1,000 \mathrm{~m}^{3}$ ( $16 \%$ of benthic drift) relative to that of winter. While drift densities for practically all taxa increased from winter to summer at Frechette Point as at all transects, 


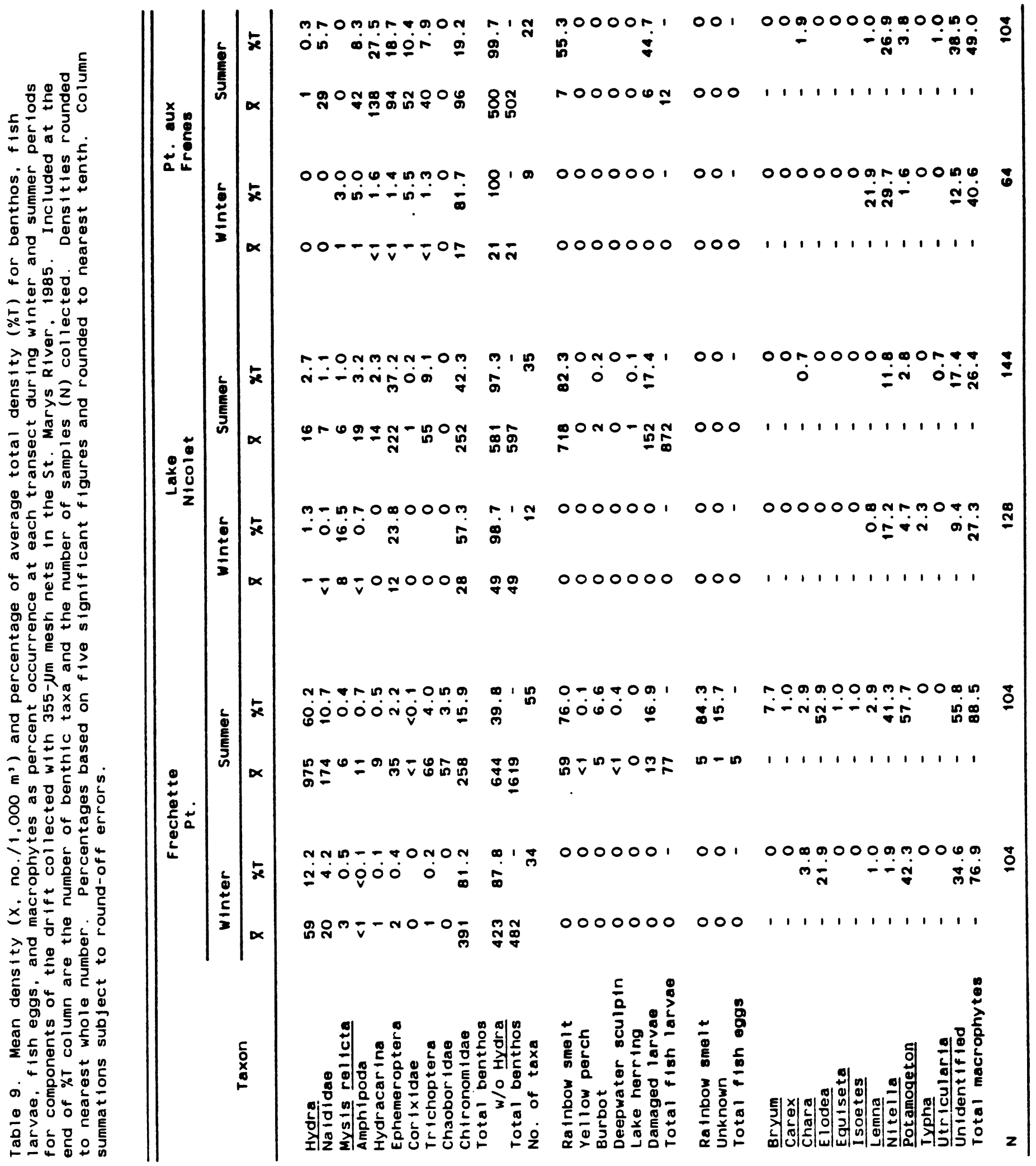


most notable increases at Frechette Point were for Hydra (975/ $1,000 \mathrm{~m}^{3}, 60 \%$ of benthic drift), Naididae $\left(174 / 1,000 \mathrm{~m}^{3}, 11 \%\right)$, Trichoptera $\left[66 / 1,000 \mathrm{~m}^{3}, 48 ;\right.$ largely Oecetis $\left.\left(51 / 1,000 \mathrm{~m}^{3}\right)\right]$, Chaoboridae $\left(57 / 1,000 \mathrm{~m}^{3}, 3.5 \%\right)$, and Ephemeroptera $\left[35 / 1,000 \mathrm{~m}^{3}\right.$, $2.2 \%$; largely Caenis $\left.\left(16 / 1,000 \mathrm{~m}^{3}\right)\right]$.

At Point aux Frenes as at Frechette Point, the percentage of Chironomidae in the benthic drift decreased from $82 \%$ (winter) to 19\% (summer) even though average density increased from $17 / 1,000$ $\mathrm{m}^{3}$ to $96 / 1,000 \mathrm{~m}^{3}$. The dominant taxon in the summer benthic drift at Point aux Frenes was Hydracarina which averaged 138/ $1,000 \mathrm{~m}^{3}$ and made up $28 \%$ of benthic drift. Remaining abundant taxa in the benthic drift included Ephemeroptera $\left[94 / 1,000 \mathrm{~m}^{3}\right.$ ( $19 \%$ of benthic drift); largely Caenis $\left(52 / 1,000 \mathrm{~m}^{3}\right.$ ) and Hexagenia $\left.\left(38 / 1,000 \mathrm{~m}^{3}\right)\right]$, Trichoptera $\left[40 / 1,000 \mathrm{~m}^{3}\right.$ (8\% of benthic drift); largely Oecetis $\left.\left(37 / 1,000 \mathrm{~m}^{3}\right)\right]$, and Naididae $(29 / 1,000$ $\mathrm{m}^{3}, 6 \%$ of benthic drift).

\section{Fish Larvae--}

Average larval fish drift density was significantly greater at Lake Nicolet $\left(872 / 1,000 \mathrm{~m}^{3}\right)$ than at Frechette Point $(77 / 1,000$ $\mathrm{m}^{3}$ ) or Point aux Frenes $\left(12 / 1,000 \mathrm{~m}^{3}\right)$, with the density at Frechette Point being significantly greater than at Point aux Frenes (Table 9). In all cases, rainbow smelt larvae were dominant, making up 70-85\% of total larval fish densities. When averaged over all samples, rainbow smelt constituted $82 \%$, burbot $0.6 \%$, lake herring $0.1 \%$, yellow perch and deepwater sculpin $<0.1 \%$, and damaged, unidentified larvae $18 \%$ of average larval $f$ ish density. All identified eggs ( $84 \%$ of total eggs) were those of rainbow smelt. Fish eggs were only collected at Frechette Point (Table 8).

\section{Macrophytes--}

Based on all samples collected during winter and summer with 355- $\mu \mathrm{m}$-mesh nets, macrophytes occurred in 50\% of all samples, with the most frequently occurring taxa being Nitella (20\% of all samples), Potamogeton (18\%), and Elodea (12\%) (Table 8 ). All remaining plant taxa occurred in $<4 \%$ of samples. There were no strong seasonal differences in percent occurrence of macrophytes (winter $=48 \%$ of all samples, summer $=57 \%$ of all samples). The three plant taxa previously noted were the dominant forms during both seasons. However, only 6 macrophytic taxa were collected in winter compared with 10 in the summer.

Notable percent occurrence differences for macrophytes were observed among transects. At Frechette Point, macrophytes comprised of nine taxa occurred in $83 \%$ of samples, while they 
were less frequent at Lake Nicolet (27\%, six taxa) and Point aux Frenes (46\%, five taxa). Additionally, dominant forms and diversity varied among transects. At Frechette Point, Potamogeton $(50 \%)$, Elodea $(37 \%)$, and Nitella $(22 \%)$ were the most frequently encountered macrophytes. At Lake Nicolet, Nitella $(14 \%)$, Potamogeton $(4 \%)$, and Typha (1\%) occurred in greatest frequency. At Point aux Frenes, macrophytes occurring most often were Nitella $(28 \%)$, Lemna $(9 \%)$, and Potamogeton (3\%).

Seasonal comparisons at Frechette Point indicated macrophytes occurred in greater frequency and diversity in the summer ( $89 \%$, nine taxa) than in winter $(77 \%$, five taxa). In addition, the dominant plants changed seasonally from Potamogeton $(42 \%)$, Elodea $(22 \%)$, and Chara $(4 \%)$ in winter to Potamogeton $(58 \%)$, Elodea $(53 \%)$, and Nitella $(41 \%)$ in summer. The most notable seasonal change for a given taxon was for Nitella (2\% vs. $41 \%$ ) (Table 8 ).

At Lake Nicolet, macrophytes were encountered in similar frequencies during each season (27\% vs. 26\%). Four plant taxa were collected during each season, but the dominant plant taxa did not change appreciably between seasons, with Nitella occurring most frequently.

Macrophytes occurred in slightly greater frequency and diversity in summer (49\%, five taxa) when compared with winter (41\%, three taxa) at Point aux Frenes. During both seasons, the most frequently occurring plant was Nitella, although in winter percent occurrence of Lemna (22\%) was quite similar to that of Nitella $(27 \%)$. Occurrence among samples for Lemna decreased considerably to $1 \%$ in the summer.

Seasonal and Transect Comparisons of Drift Rates and Drift Intensities

Frechette Point--

The input of water from Lake Superior to the head of the St. Marys River is apportioned so that $76 \%$ flows down the western side of Sugar Island and $24 \%$ flows into Lake George (Liston et al. 1986). The portion going into Lake George flows southward above St. Joseph Island, then eastward around the island, thereby not returning to the original $76 \%$ flowing west of sugar Island (Don Williams, personal communication, U.S. Army Corps of Engineers, Detroit District). During the 23-24 February 1985 period when drift samples were collected at Frechette Point, flow at the head of the river was $1,940 \mathrm{~m}^{3} / \mathrm{s}$. Based on a $76 \%$ diversion, $1,490 \mathrm{~m}^{3} / \mathrm{s}$ were expected to have passed our Frechette Point transect during this period. However, based on our current velocity and areal approximations for subsections of a cross- 
section of the river, the discharge rate was $1,030 \mathrm{~m}^{3} / \mathrm{s}$. Due to this difference, two estimates for drift rate were determined. The first is the "calculated" drift rate which is based on our measurements and will be referred to in the ensuing text simply as drift rate(s). The second is the "adjusted" drift rate which is our calculated values adjusted upward or downward to reflect the difference between expected and calculated discharge at a given transect and season. When referring to the latter drift rate, it will be referred to specifically as "adjusted" drift $\operatorname{rate}(s)$.

Based on our winter discharge values, $49 \mathrm{~m}^{3} / \mathrm{s}(4.8 \%$ of total) was discharged along the western shoreline (stations 1-3) at Frechette Point, $69 \mathrm{~m}^{3} / \mathrm{s}(6.7 \%$ of total) along the eastern shoreline (stations 5-7), and $913 \mathrm{~m}^{3} / \mathrm{s}(88.6 \%$ of total) in the navigation channel (station 4) (Table 10). The winter benthic drift rate for the entire transect was $49.0 \times 10 \% / 24 \mathrm{~h}$ $($ "adjusted" $=70.9 \times 10 \% / 24 \mathrm{~h})$. Along the western shoreline, the benthic drift rate was $2.84 \times 10 \% / 24 \mathrm{~h}(5.8 \%$ of total). The benthic drift rate was lowest along the eastern shoreline $(0.19 \mathrm{x}$ $10 \% / 24 \mathrm{~h}, 0.4 \%$ of total). Greatest drift rate occurred in the navigation channel $(46.0 \times 10 \% / 24 \mathrm{~h}, 93.9 \%$ of total).

Drift intensity [see METHODS and Waters (1972) for details] was similar along the western shoreline $(57,959)$ and the navigation channel $(50,383)$ but was very low on the eastern shoreline $(2,754)$. Overall, winter drift intensity at Frechette Point was 47,573 .

As only an average monthly discharge value was available to us for June $\left(1,917 \mathrm{~m}^{3} / \mathrm{s}\right), 76 \%$ of this value $\left(1,457 \mathrm{~m}^{3} / \mathrm{s}\right)$ was used as the expected discharge at Frechette Point as well as at Lake Nicolet and Point aux Frenes. Our calculated discharge estimate during June at Frechette Point was $1,217 \mathrm{~m}^{3} / \mathrm{s}$. The percentage of discharge apportioned to the river subsections during summer at Frechette Point was similar to that of winter (Table 10). The benthic drift rate ior the entire transect was $151.3 \mathrm{x} 10 \% / 24 \mathrm{~h}$ ("adjusted" $=181.4 \times 10 \% / 24 \mathrm{~h})$, while the drift rate for larval Iish was $1.87 \times 10^{6} / 24 \mathrm{~h}$ ("adjusted" $=2.71 \times 10 \% / 24 \mathrm{~h}$ ).

Greatest seasonal changes in benthic drift rates at Frechette Point occurred along the eastern shoreline where the drift rate of $18.2 \times 10 \% / 24 \mathrm{~h}$ ( $12 \%$ of total) represented a 96fold increase when compared with winter (Table 10). Along the western shoreline, the benthic drift rate was $8.13 \times 10 \% / 24 \mathrm{~h}$ (5.4\% of total), while that for larval fish was $0.35 \times 10 \% / 24 \mathrm{~h}$ (18.7\% of total). In the navigation channel, benthic drift rate ( $124.9 \times 10 \% / 24 \mathrm{~h}, 82.6 \%$ of total) and larval $\mathrm{fish}$ drift rate $(1.43 \times 10 \% / 24 \mathrm{~h}, 76.5 \%$ of total) were greater than those observed along either shoreline. 


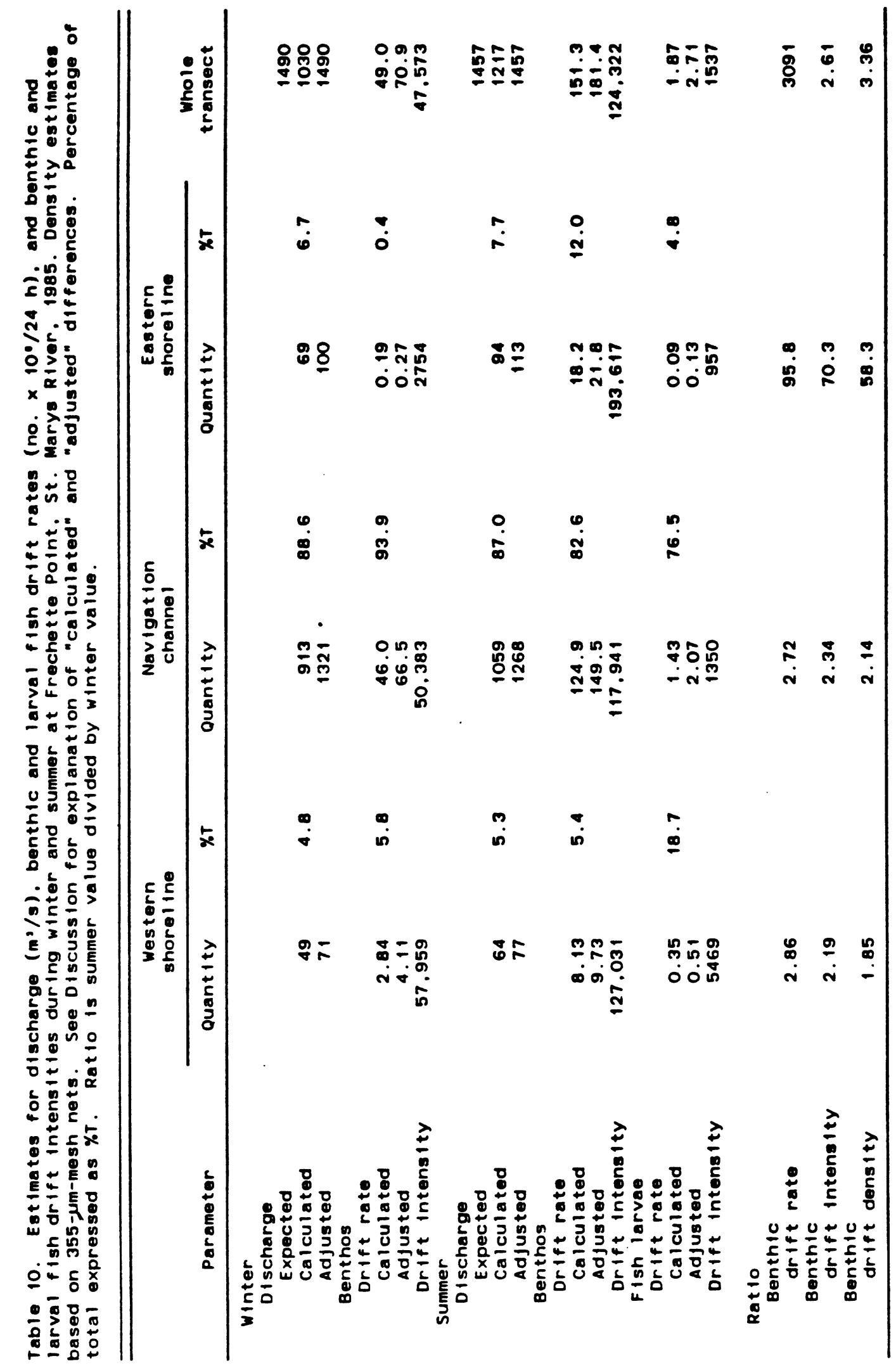


Benthic drift intensity was greatest on the eastern side of the river $(193,617)$, but larval $f$ ish drift rate $(0.09 \times 10 \% / 24 \mathrm{~h}$, $4.8 \%$ of total) and drift intensity (957) were the lowest among the three subsections of the river at Frechette Point. While benthic drift intensity along the western shoreline $(127,031)$ was similar to that of the navigation channel $(117,941)$, larval $f$ ish drift intensity was much greater on the western shoreline $(5,469)$ when compared with the navigation channel $(1,350)$ and the eastern shoreline. Overall, larval fish drift intensity for the transect was 1,537, while that for benthos was 124,322. For the benthos, this drift intensity represented a $261 \%$ increase from winter to summer (Table 10).

\section{Lake Nicolet--}

Discharge was $2,005 \mathrm{~m}^{3} / \mathrm{s}$ at the head of the river during the 2-7 March 1985 period when drift samples were collected at the lake Nicolet transect. Based on a $76 \%$ apportionment of flow, we expected a discharge of $1,524 \mathrm{~m}^{3} / \mathrm{s}$ at our Lake Nicolet transect. Our discharge calculation was $1,578 \mathrm{~m}^{3} / \mathrm{s}$. Percent discharge attributable to each river subsection was fairly similar (Table 11). The benthic drift rate for the entire transect during winter was $2.83 \times 10 \% / 24 \mathrm{~h}$ ("adjusted" $=2.73 \times 10 \% / 24 \mathrm{~h}$ ). Greatest benthic drift rates were calculated for the eastern (stations 7-9) $(0.93 \times 10 \% / 24 \mathrm{~h}, 32.9 \%$ of total) and middle (station 5) $(0.82 \times 10 \% / 24 \mathrm{~h}, 29.0 \%$ of total) subsections of the river. The lowest benthic drift rate was calculated for the upbound channel $(0.21 \times 10 \% / 24 \mathrm{~h}, 7.4 \%$ of total). Benthic drift rates were $0.32 \times 10 \% / 24 \mathrm{~h}$ (11.3\% of total) on the western shore and $0.55 \times 10 \% / 24 \mathrm{~h}$ (19.4\% of total) in the downbound channel (Table 1l).

Greatest benthic drift intensity occurred on the eastern shoreline $(3,310)$ and least in the upbound channel (857). Benthic drift intensities in remaining subsections of the river were similar, ranging from 1,441 to 1,687 . Overall, benthic drift intensity for the transect during winter was 1,793 (Table 11).

As at Frechette Point, expected discharge at our Lake Nicolet transect during June was $1,457 \mathrm{~m}^{3} / \mathrm{s}$. Based on our measurements, we calculated a discharge of $1,282 \mathrm{~m}^{3} / \mathrm{s}$. Percent distribution of the water mass attributable to each subsection of the river varied little seasonally. The benthic drift rate for the entire Lake Nicolet transect during June was $29.0 \times 10 \% / 24 \mathrm{~h}$ ("adjusted" $=33.0 \times 10 \% / 24 \mathrm{~h})$. Relative to the winter, benthic drift rate increased by a factor of 10.2 in summer (Table 11). In all comparisons of summer and winter benthic drift rates within each river subsection, those in summer were greater. Summer benthic drift rates were lowest among the shorelines 


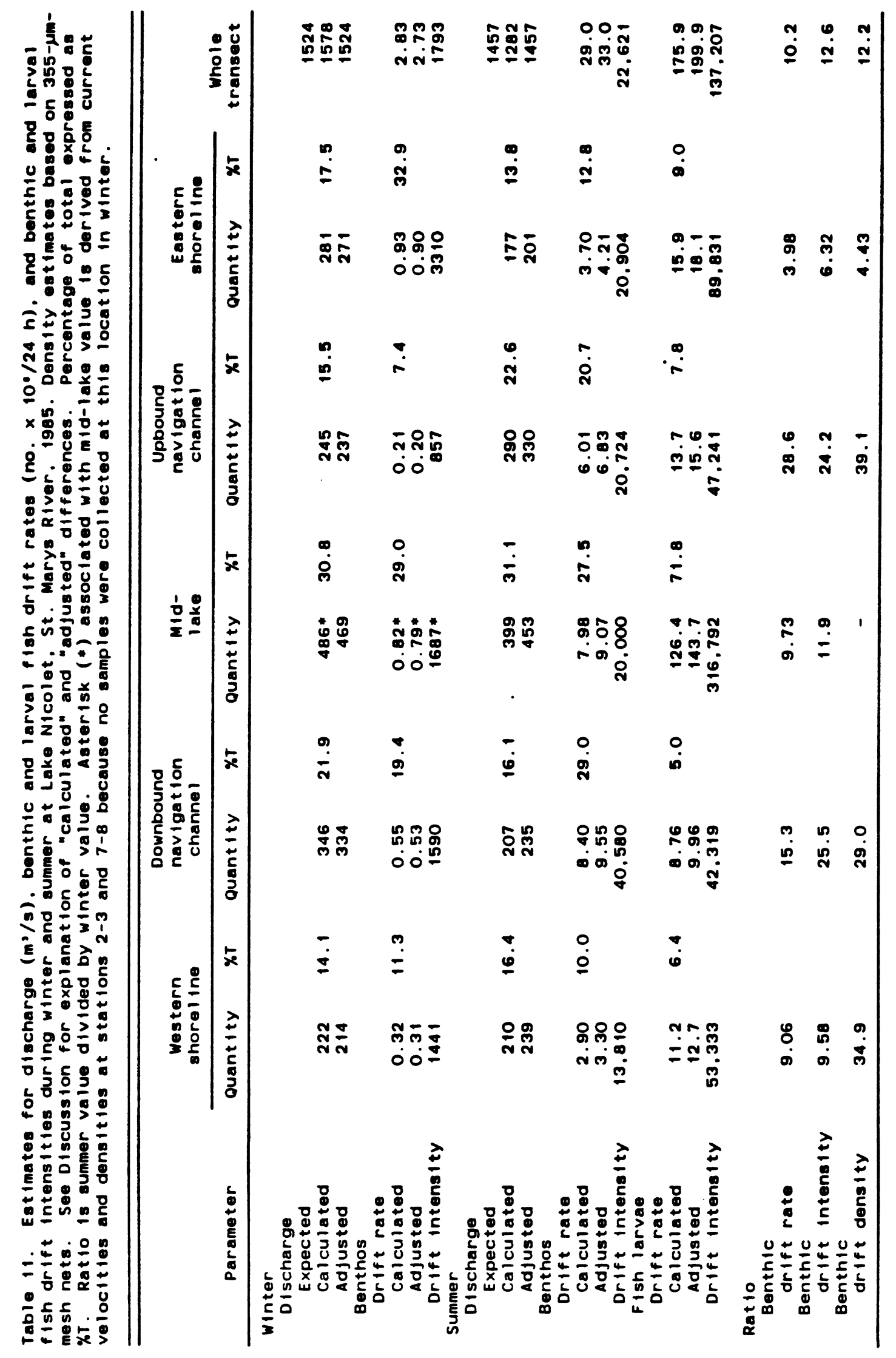


$(2.90-3.70 \times 10 \% / 24 \mathrm{~h})$ and were similarly high in remaining subsections $(6.01-8.40 \times 10 \% / 24 \mathrm{~h})$. Most notable increases in the percentage of total benthic drift rate were for the two navigation channels. In the downbound channel, the percentage of total drifting benthos increased from $19.4 \%$ in winter to $29.0 \%$ in summer. In the upbound channel, the increase was from $7.4 \%$ in winter to $20.7 \%$ in summer. Consequently, during winter $26.8 \%$ of drifting benthos was in the two channels, while during summer the portion of drifting benthos in the two channels increased to 49.7\%. The most notable decrease occurred along the eastern shoreline where the percentage of the total benthic drift rate decreased from $32.9 \%$ in winter to $12.8 \%$ in summer. Regardless of benthic drift rate differences among river subsections, benthic drift intensities were very similar in all subsections $(13,810-$ 20,904 ) except in the downbound channel where benthic drift intensity was 40,500 . The benthic drift intensity for the entire Lake Nicolet transect was 22,621 which represented an increase by a factor of 12.6 compared with winter.

Larval fish drift rate during summer was $175.9 \times 10^{6} / 24 \mathrm{~h}$ ("adjusted" $=199.9 \times 10 \% / 24 \mathrm{~h})$. Seventy-two percent $(126.4 \mathrm{x}$ $10^{\circ} / 24 \mathrm{~h}$ ) of the total larval fish drift rate originated in the middle portion of Lake Nicolet (station 5). Larval fish drift rates in other subsections of the river were similar and much lower than in the middle subsection ranging from $8.76 \times 10 \% / 24 \mathrm{~h}$ to $15.9 \times 10 \% / 24 \mathrm{~h}$ (5-9\% of total).

For the transect as a whole, larval fish drift intensity was 137,207. However, all values except that for the middle portion of the river were much lower than that for the whole transect ranging from 42,319 to 89,831 . Larval fish drift intensity for the middle portion of the river was very large $(316,792)$ (Table 11).

Point aux Frenes--

Winter drift rates and intensities were not calculated at Point aux Frenes since only stations $1-4$ could be sampled. During summer, the expected discharge at Point aux Frenes was the same as that used at Frechette Point and Lake Nicolet $\left(1,457 \mathrm{~m}^{3} /\right.$ s). Based on our measurements and an average navigation channel depth of $10 \mathrm{~m}$, we calculated a discharge of $2,248 \mathrm{~m}^{3} / \mathrm{s}$, with $67.7 \%\left(1,509 \mathrm{~m}^{3} / \mathrm{s}\right)$ flowing down the navigation channel, $17.2 \%$ $\left(384 \mathrm{~m}^{3} / \mathrm{s}\right)$ along the western shoreline, and $15.4 \%\left(345 \mathrm{~m}^{3} / \mathrm{s}\right)$ along the eastern shoreline. The benthic drift rate at Point aux Frenes was $2.39 \times 10 \% / 24 \mathrm{~h}$ ("adjusted" $=15.9 \times 10^{\circ} / 24 \mathrm{~h}$ ). With respect to river subsections, benthic drift rates decreased eastward, with $9.67 \times 10 \% / 24 \mathrm{~h}(40.4 \%$ of total) on the western shore, $8.22 \times 10 \% / 24 \mathrm{~h}(34.4 \%$ of total) in the channel, and 6.02 $\mathrm{x} 10 \% / 24 \mathrm{~h}(25.2 \%$ of total) on the eastern shore (Table 12). 


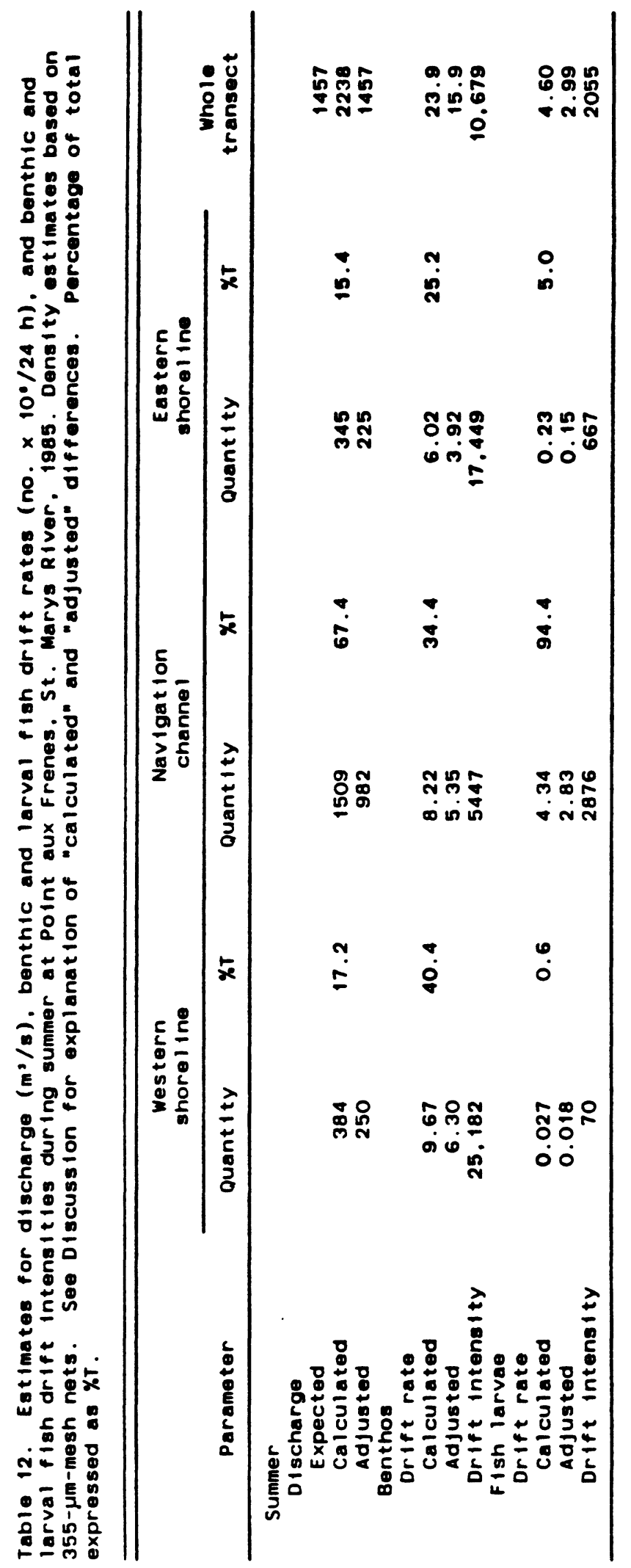


Benthic drift intensity for the entire transect was 10,679. Highest benthic drift intensity occurred on the eastern shore $(25,182)$ and lowest in the channel $(5,447)$ (Table 12).

Larval $f$ ish drift rate for the entire transect was $4.60 \mathrm{x}$ $10 \% / 24 \mathrm{~h}$ ("adjusted" $=2.99 \times 10 \% / 24 \mathrm{~h})$. Maximum larval $\mathrm{f}$ ish drift rate and drift intensity occurred in the navigation channel [4.34 x $10 \% / 24 \mathrm{~h}(94.4 \%$ of total) and 2,876 , respectively]. Although benthic drift rate was greatest on the western shore, larval $f$ ish drift rate was lowest $(0.027 \times 10 \% / 24 \mathrm{~h}, 0.6 \%$ of total). In addition, larval fish drift intensity was very low (70). Although higher than along the western shore, larval fish drift rate and drift intensity were still both low on the eastern shore $[0.23 \times 10 \% / 24 \mathrm{~h}(5.0 \%$ of total) and 667 , respectively] (Table 12).

\section{Diel Comparisons of Drift Densities}

\section{Benthos--}

Comparisons of diel benthic drift densities during both winter and summer at each transect indicated night abundances were significantly greater than daytime densities for all comparisons except at Frechette Point in summer. In this latter comparison, daytime benthic density $\left[2,488 / 1,000 \mathrm{~m}^{3}\right.$; largely Hydra (74\%)] was significantly greater than the night benthic density estimate $\left(750 / 1,000 \mathrm{~m}^{3}\right)$. Among comparisons having significantly greater night densities, abundances at night increased over day densities from a minimum of $882 \%$ to a maximum of $2,198 \%$. At each transect, the number of taxa collected was greatest in night samples regardless of season.

Daytime benthic drift density at Frechette Point was distinguished from that at Lake Nicolet and Point aux Frenes during both winter and summer due to a large component attributable to Hydra (Table 13). Daytime winter drift at Frechette Point averaged $97 / 1,000 \mathrm{~m}^{3}$ and was comprised primarily of Hydra $\left(54 / 1,000 \mathrm{~m}^{3}\right)$, Chironomidae $\left(26 / 1,000 \mathrm{~m}^{3}\right)$, and Naididae $\left(13 / 1,000 \mathrm{~m}^{3}\right)$. Similar estimates at $\mathrm{night}$ averaged $866 / 1,000 \mathrm{~m}^{3}$, with Chironomidae $\left(756 / 1,000 \mathrm{~m}^{3}\right)$, Hydra $\left(64 / 1,000 \mathrm{~m}^{3}\right)$, and Naididae $\left(28 / 1,000 \mathrm{~m}^{3}\right)$ being the most numerous drifting benthos. During summer, daytime benthic drift averaged $2,488 / 1,000 \mathrm{~m}^{3}$ and was dominated by Hydra $\left(1,836 / 1,000 \mathrm{~m}^{3}\right)$, Naididae $\left(303 / 1,000 \mathrm{~m}^{3}\right)$, and Chironomidae $\left(244 / 1,000 \mathrm{~m}^{3}\right)$. At night, average benthic drift density $\left(750 / 1,000 \mathrm{~m}^{3}\right)$ decreased with Chironomidae $(272 / 1,000$ $\left.\mathrm{m}^{3}\right)$, Trichoptera [126/1,000 $\mathrm{m}^{3}$; largely Oecetis $\left.\left(102 / 1,000 \mathrm{~m}^{3}\right)\right]$, Hydra $\left(144 / 1,000 \mathrm{~m}^{2}\right)$, and Chaoboridae $\left(87 / 1,000 \mathrm{~m}^{3}\right)$ the dominart taxa. 


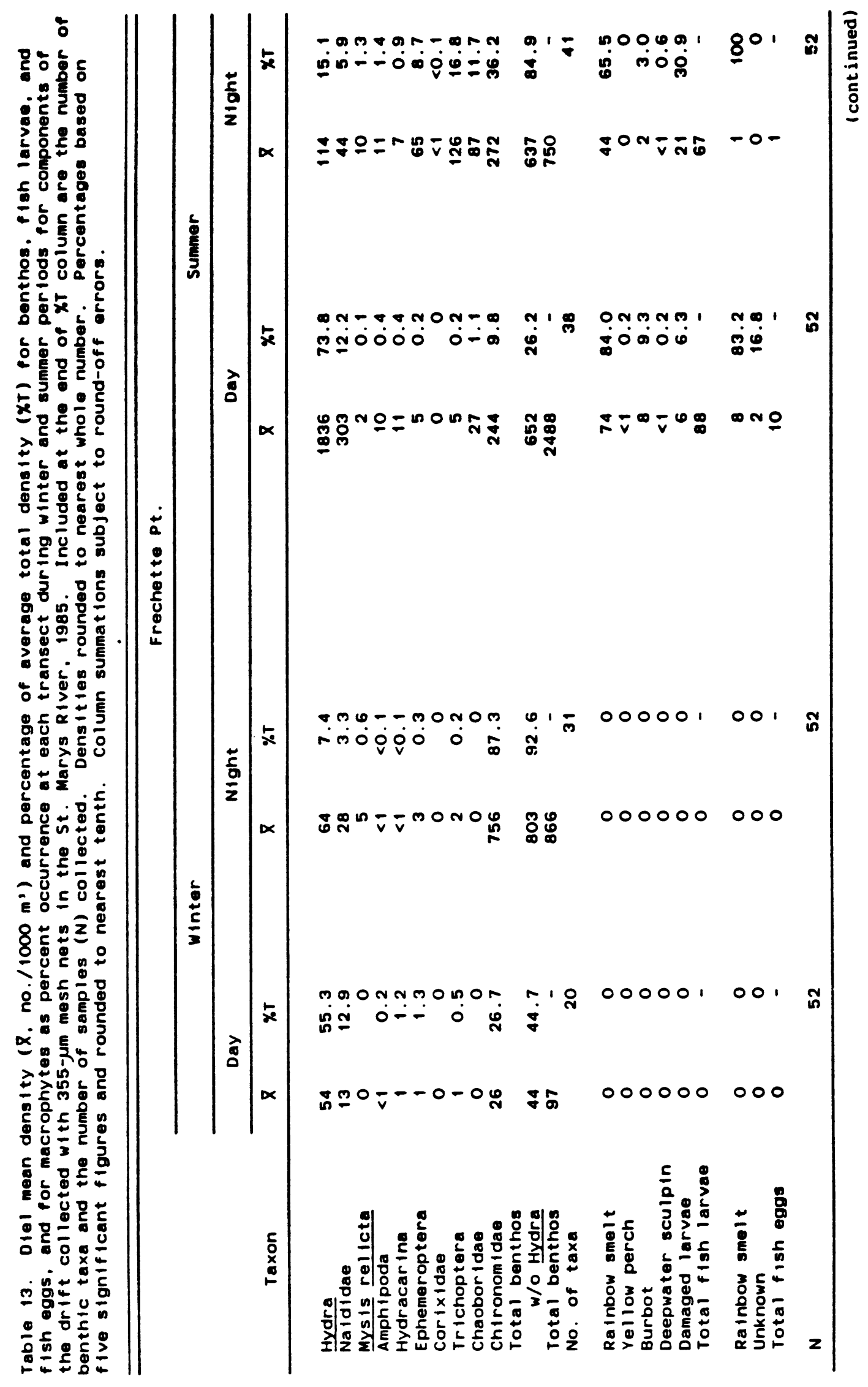




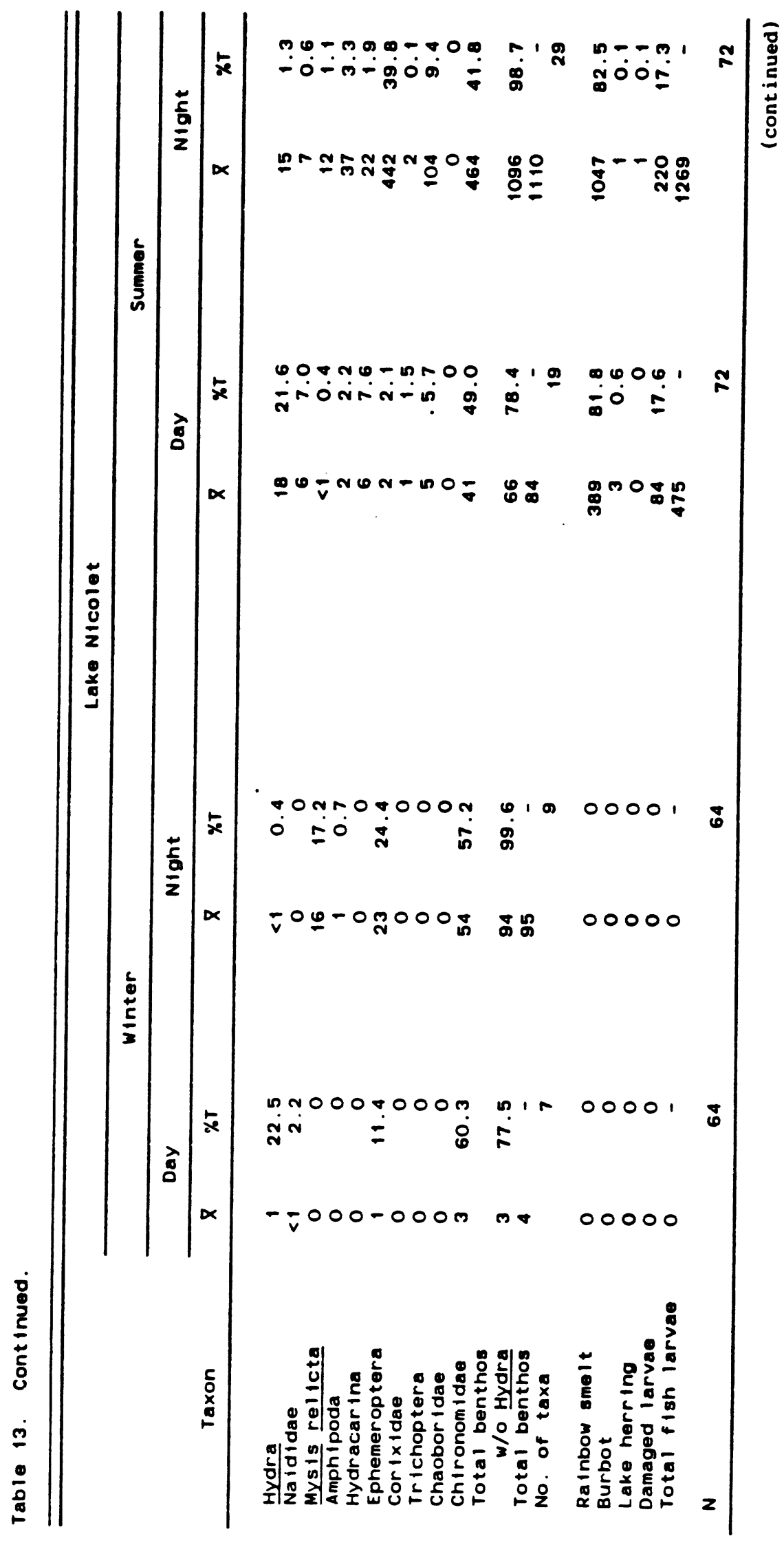




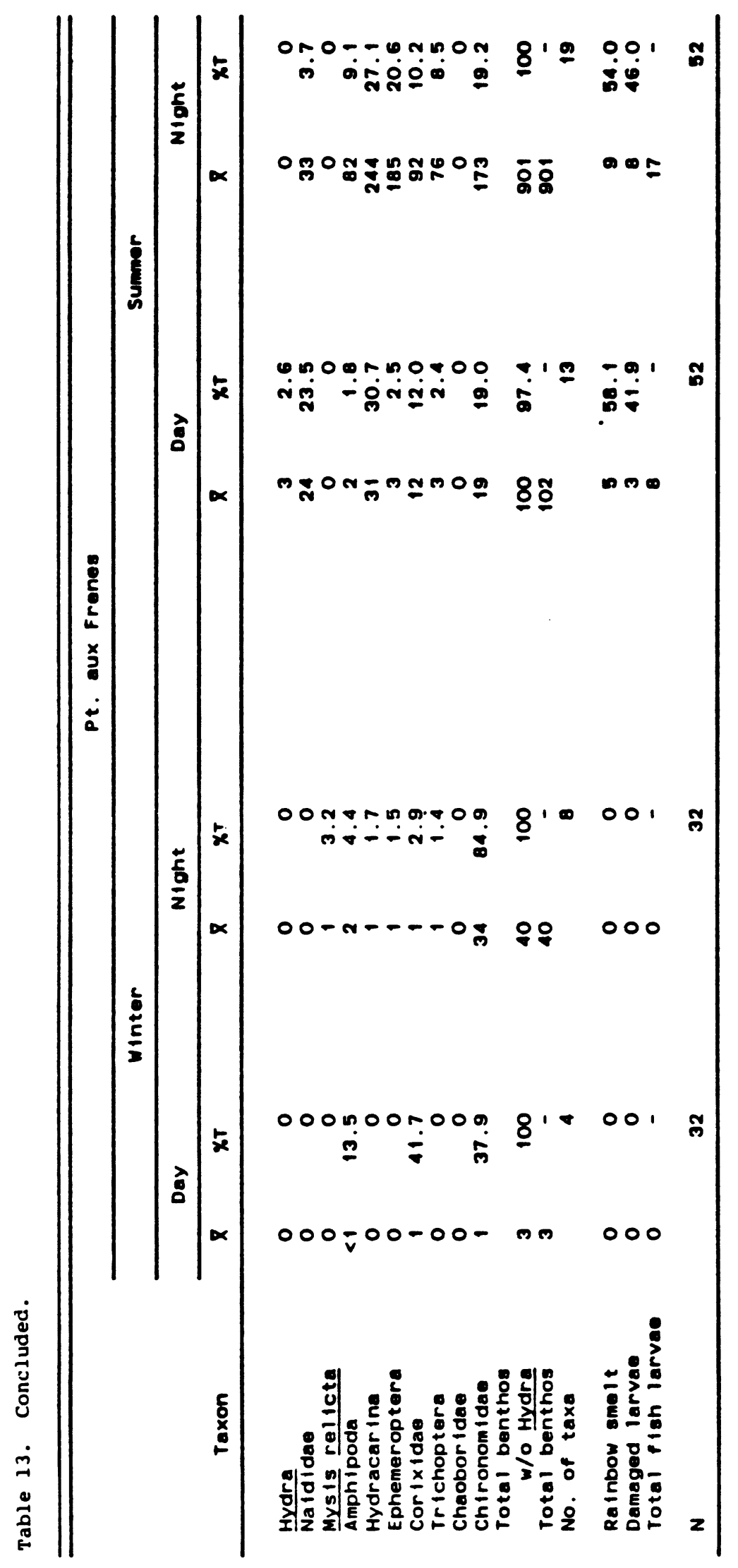


At Lake Nicolet, average daytime winter benthic density was low $\left(4.3 / 1,000 \mathrm{~m}^{3}\right)$, with the dominant taxa being Chironomidae $\left(2.6 / 1,000 \mathrm{~m}^{3}\right), \operatorname{Hydra}\left(1.0 / 1,000 \mathrm{~m}^{3}\right)$, and Ephemeroptera $(0.5 /$ $\left.1,000 \mathrm{~m}^{3}\right)$. At night, benthic drift density increased to $95 / 1,000$ $\mathrm{m}^{3}$. While Chironomidae remained the dominant taxon $(54 / 1,000$ $\left.\mathrm{m}^{3}\right)$, Ephemeroptera made up $24 \%$ and Mysis relicta $17 \%$ of benthic drift (Table 13).

Lake Nicolet summer daytime benthic drift abundance averaged $84 / 1,000 \mathrm{~m}^{3}$ and was dominated by Chironomidae $\left(41 / 1,000 \mathrm{~m}^{3}\right)$ and Hydra $\left(18 / 1,000 \mathrm{~m}^{3}\right)$. Average summer benthic drift increased considerably at night $\left(1,110 / 1,000 \mathrm{~m}^{3}\right)$, with Chironomidae (464/ 1,000 $\left.\mathrm{m}^{3}\right)$, Ephemeroptera $\left(442 / 1,000 \mathrm{~m}^{3}\right)$, and Trichoptera (104/ $1,000 \mathrm{~m}^{3}$ ) being the most abundant components. For mayflies in summer night drift, Hexagenia was the most numerous averaging $310 / 1,000 \mathrm{~m}^{3}$. Among caddisflies, Oecetis was the most abundant averaging $93 / 1,000 \mathrm{~m}^{3}$.

At Point aux Frenes, average winter benthic drift abundance increased from $3 / 1,000 \mathrm{~m}^{3}$ in the daytime to $40 / 1,000 \mathrm{~m}^{3}$ at $\mathrm{night}$. A similar diel trend was observed during the summer where average benthic drift increased from $102 / 1,000 \mathrm{~m}^{3}$ during the day to $901 /$ $1,000 \mathrm{~m}^{3}$ at night. Chironomidae and Corixidae were the most abundant taxa $\left(1 / 1,000 \mathrm{~m}^{3}\right)$ in daytime winter benthic drift samples; whereas, the night estimate was dominated by Chironomidae $\left(34 / 1,000 \mathrm{~m}^{3}\right)$ (Table 13$)$.

During summer at Point aux Frenes for both day and night, Chironomidae made up only $19 \%$ of benthic drift which averaged $102 / 1,000 \mathrm{~m}^{3} / \mathrm{s}$ during the day and $901 / 1,000 \mathrm{~m}^{3} / \mathrm{s}$ at night (Table 13). The most abundant drifting taxa during the day were Hydracarina $\left(31 / 1,000 \mathrm{~m}^{3}\right)$, Naididae $\left(24 / 1,000 \mathrm{~m}^{3}\right)$, and Corixidae $\left(12 / 1,000 \mathrm{~m}^{3}\right)$. At night, Hydracarina remained the most numerous drifting taxon collected averaging 244/1,000 $\mathrm{m}^{3}$. Other major components included Ephemeroptera $\left[85 / 1,000 \mathrm{~m}^{3}\right.$; largely Caenis $\left(102 / 1,000 \mathrm{~m}^{3}\right)$ and Hexagenia $\left.\left(76 / 1,000 \mathrm{~m}^{3}\right)\right]$, Corixidae $(92 / 1,000$ $\left.\mathrm{m}^{3}\right)$, Amphipoda $\left(82 / 1,000 \mathrm{~m}^{3}\right)$, and Trichoptera $\left[76 / 1,000 \mathrm{~m}^{3}\right.$; largely Oecetis $\left(75 / 1,000 \mathrm{~m}^{3}\right)$ ].

\section{Fish Larvae--}

Diel larval fish drift densities were significantly different only at Lake Nicolet where densities during the night $\left(1,269 / 1,000 \mathrm{~m}^{3}\right)$ exceeded day densities $\left(475 / 1,000 \mathrm{~m}^{3}\right)$ by a factor or 2.67 (Table 13). At Frechette Point, average day larval $\mathrm{fish}$ drift density $\left(88 / 1,000 \mathrm{~m}^{3}\right)$ exceeded night density $\left(67 / 1,000 \mathrm{~m}^{3}\right)$, but the difference was not significant. At Point aux Frenes, night density of larval $\mathrm{fish}$ drift $\left(17 / 1,000 \mathrm{~m}^{3}\right)$ was twice that of daytime density, but the difference was not

significant. 
Macrophytes--

When examining diel differences pooled over all transects and stations, percent occurrence of macrophytes was low. Macrophytes occurred in $54 \%$ of day samples and $46 \%$ of night samples. Dominant plant taxa were Potamogeton (23\%), Nitella (22\%), and Elodea $(14 \%)$ in day samples; whereas, at night dominant forms were Nitella (19\%), Potamogeton (14\%), and Elodea (10\%). The number of taxa collected during day and night samples was the same (nine taxa), with two taxa encountered only during the day and another two only at night.

\section{Depth Strata Comparisons of Drift Densities}

Frechette Point--

Nearly all estimates of benthic drift density at all transects, regardless of mesh size, had lowest abundances at the surface and greatest at the bottom. However, during winter at Frechette Point, benthic drift was greatest near bottom (630) $\left.1,000 \mathrm{~m}^{3}\right)$ and least at mid-depth $\left(289 / 1,000 \mathrm{~m}^{3}\right)$. In spite of this deviation from the general trend, there were no significant density differences among the three depth strata estimates of total benthic drift density during winter.

Regardless of water column depth during the winter at Frechette Point, benthic drift was dominated by Chironomidae (8185\%) (Table 14). Remaining taxa contributing substantively to the drift were Naididae and Hydra which cumulatively made up 12$17 \%$ of the drifting benthos.

Summer benthic drift density estimates among depth strata sampled were significantly different. The surface estimate of total benthic drift density $\left(135 / 1,000 \mathrm{~m}^{3}\right)$ was significantly lower than either mid-depth $\left(490 / 1,000 \mathrm{~m}^{3}\right)$ or bottom $(827 / 1,000$ $\mathrm{m}^{3}$ ) estimates, which were not significantly different.

At all depth strata, Hydra was the dominant taxon during summer, making up 57-92\% of the drifting benthos. The percentage of total drifting benthos attributable to Hydra decreased from surface to bottom even though density increased. As during the winter only two other taxa contributed moderate numbers to the drifting benthos; Naididae and Chironomidae. These two taxa combined made up 17-28\% of total benthic drift density, with densities increasing from surface to bottom. During winter and summer, greatest densities of Mysis relicta occurred in surface waters and decreased from surface to bottom. Finally, during both winter and summer, the number of taxa collected was least at the surface ( 7 taxa, winter) and greatest at the bottom (5l taxa, summer). 


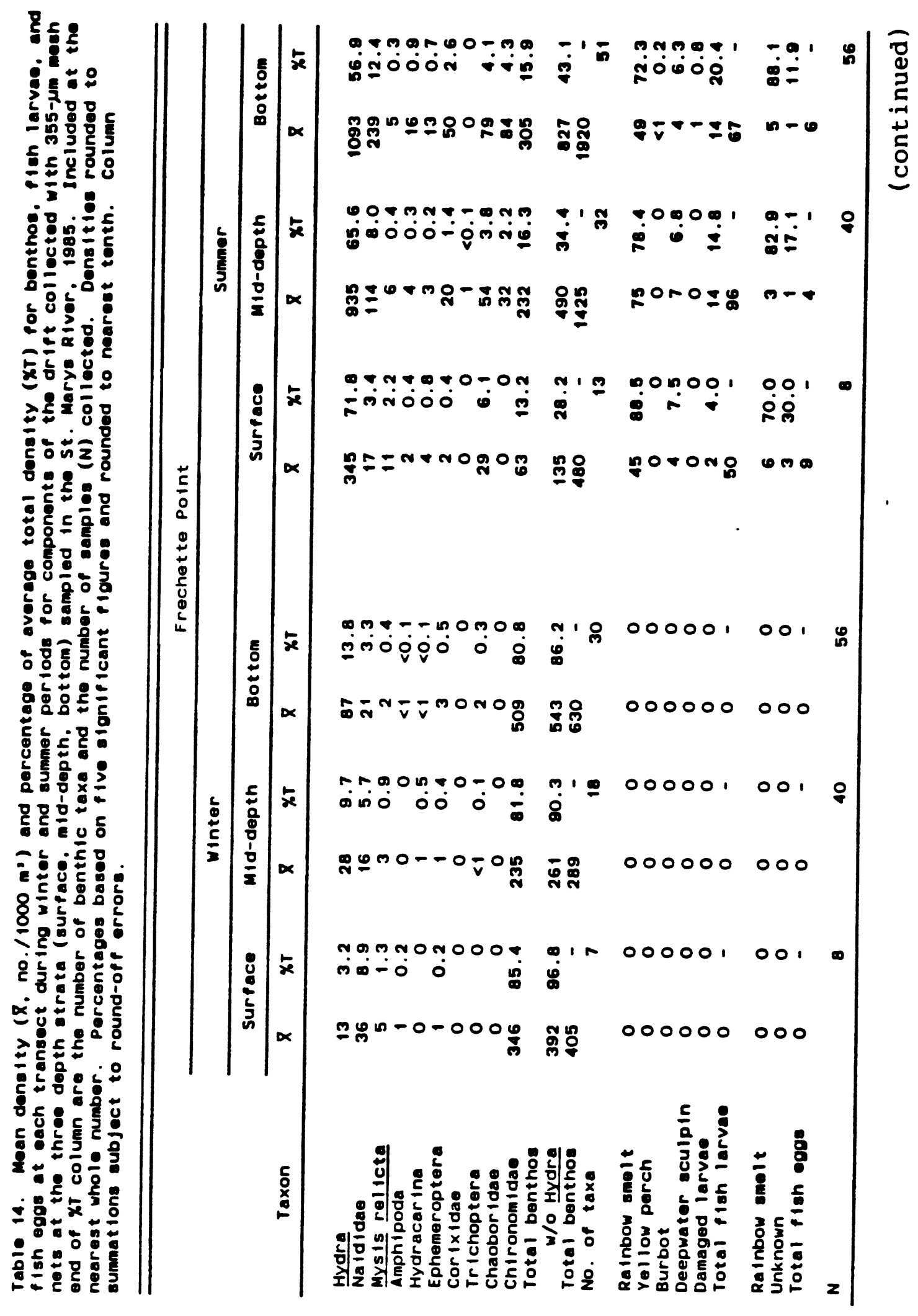




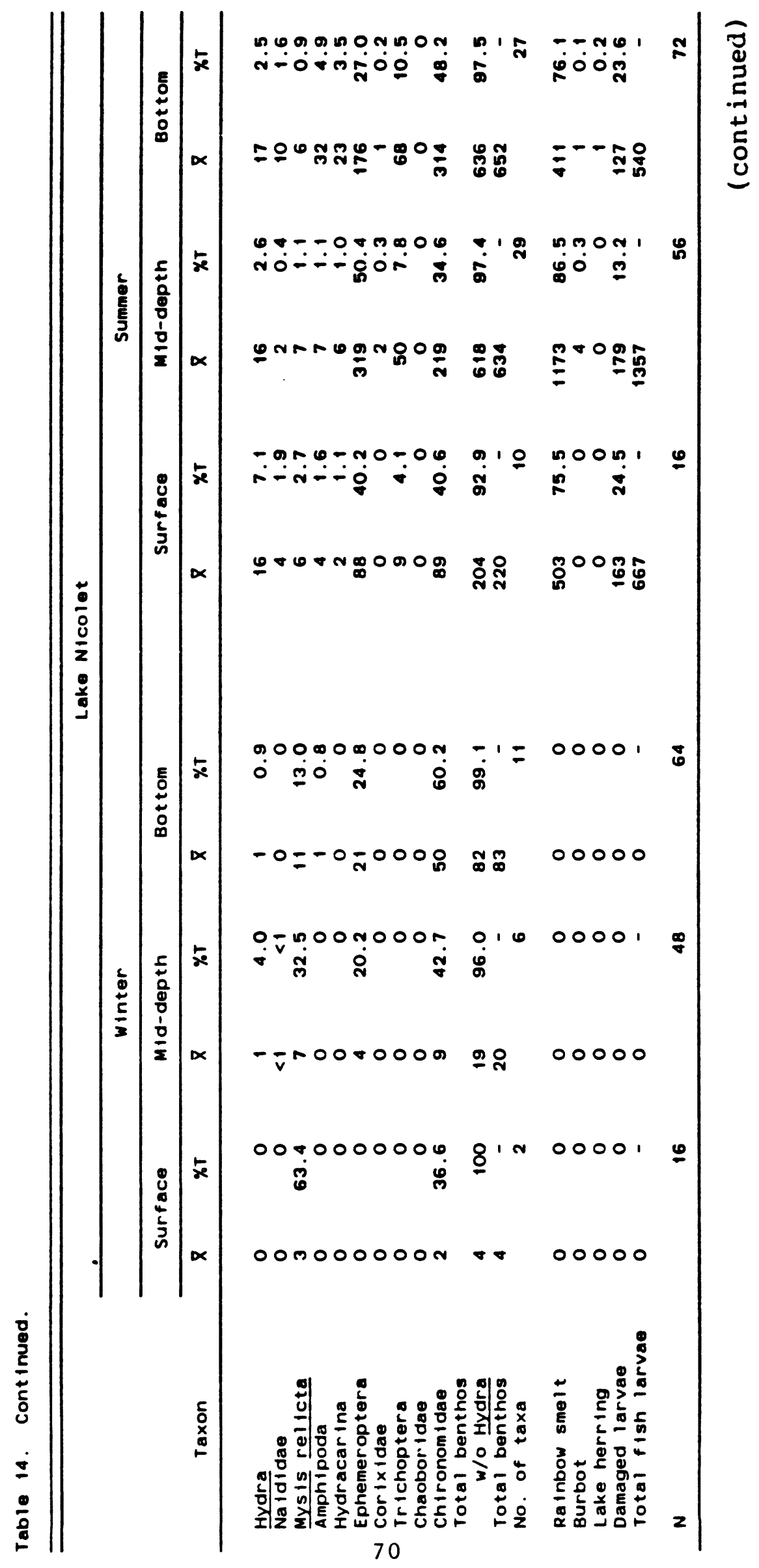




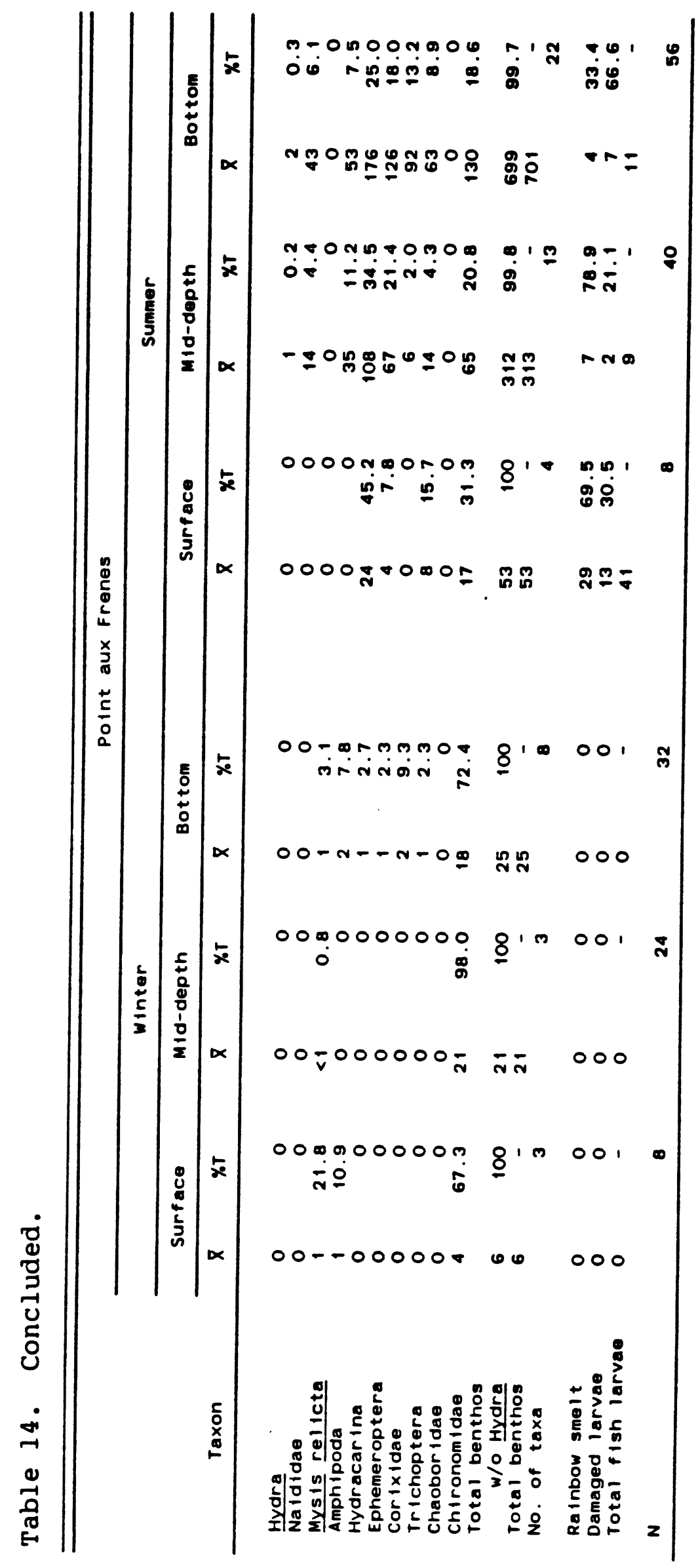


There were two general trends with respect to drifting benthos and depth strata. First, benthic drift density was dominated by Chironomidae in winter and Hydra in summer, regardless of depth strata. Second, density and diversity of the drifting benthos were greatest near bottom during both seasons.

There were no significant larval fish drift density differences among depth strata sampled. Greatest numbers of $f$ ish larvae were collected at mid-depth $\left(96 / 1,000 \mathrm{~m}^{3}\right)$; the least occurred at the surface $\left(50 / 1,000 \mathrm{~m}^{3}\right)$.

Lake Nicolet--

Benthic drift density and diversity at Lake Nicolet were considerably reduced at all depths when compared with those at Frechette Point. However, both parameters had similar trends to those at Frechette Point with increasing abundance and diversity from surface to bottom regardless of season (Table 14). However, only during winter were density estimates of total benthic drift significantly different, with the bottom $\left(83 / 1,000 \mathrm{~m}^{3}\right)$ and surface $\left(4 / 1,000 \mathrm{~m}^{3}\right)$ estimates being different. The mid-depth density estimate $\left(20 / 1,000 \mathrm{~m}^{3}\right)$ was not significantly different from surface or bottom estimates.

The winter surface estimate of drifting benthos was dominated by $M$. Ielicta $\left(63 \%\right.$, but only $\left.2.6 / 1,000 \mathrm{~m}^{3}\right)$ and Chironomidae. At mid-depth, while abundance of $M$. relicta increased to $6.5 / 1,000 \mathrm{~m}^{3}$, its percentage of drifting benthos decreased to $33 \%$ due to increases in other components of the benthos, largely Chironomidae and Ephemeroptera. At bottom, $M$. relicta increased to a maximum of $10.8 / 1,000 \mathrm{~m}^{3}$, but its percentage of drifting benthos continued to decrease to $13 \%$; once again due to increases in chironomids and ephemeropterans.

During summer, density and diversity estimates were considerably greater than during winter. However, regardless of depth, the chironomids and ephemeropterans made up 75-85\% of total benthic drift density. Mysis relicta occurred in densities similar to those of winter, ranging from $5.6 / 1,000 \mathrm{~m}^{3}$ to $6.7 /$ $1,000 \mathrm{~m}^{3}$ (Table 14).

The general pattern of drifting benthos with respect to depth at Lake Nicolet was one of increasing density and diversity from surface to bottom depth strata. Winter estimates of drifting benthos were dominated by chironomids and mysids at the surface and these two taxa plus ephemeropterans at remaining depths. Summer estimates of these two parameters were greater than winter estimates and were dominated by chironomids and ephemeropterans, with densities of mysids being similar at all depth strata. 
Comparisons of larval $\mathrm{fish}$ drift at Lake $\mathrm{Nicolet}$ indicated mid-depth $\left(1,357 / 1,000 \mathrm{~m}^{3}\right)$ and surface $\left(667 / 1,000 \mathrm{~m}^{3}\right)$ densities were significantly greater than at the bottom $\left(540 / 1,000 \mathrm{~m}^{3}\right)$. There was no significant difference between mid-depth and surface density estimates of larval fish drift.

\section{Point aux Frenes--}

As with Lake Nicolet and Frechette Point, benthic density and diversity increased with increasing depth strata and overall were greater in summer when compared with winter. Winter benthic density and diversity estimates at Point aux Frenes were most similar to those at Lake Nicolet. Comparisons of depth strata estimates for total benthic drift density were non-significant. Chironomidae was the dominant taxon, making up 67-98\% of total benthic drift density (Table 14). Although $M$. relicta comprised $22 \%$ of the surface total benthic drift density, average density was only $1.4 / 1,000 \mathrm{~m}^{3}$, with even lower abundances at remaining depth strata. Other components of the benthos, mostly Corixidae (9.3\% of benthic drift) and Amphipoda (7.8\%), made up moderate amounts of benthic drift only at the bottom depth stratum.

During summer, total benthic drift densities on the bottom and at mid-depth were significantly greater than at the surface. Hyaracarina was the most numerous taxon encountered in the drifting benthos at each depth strata. Chironomidae and Ephemeroptera were the second- and third-most abundant taxa in the drifting benthos at mid-depth and bottom. No mysids were collected during the summer at Point aux Frenes.

The general trend for drifting benthos at Point aux Frenes was for density and diversity to increase from surface to bottom depth strata during both seasons. Winter benthic drift was dominated by Chironomidae at all strata, while in summer drifting benthos, Hydracarina, Chironomidae, and Ephemeroptera were the most numerous benthic forms.

At Point aux Frenes, larval $f$ ish drift density was significantly greater at the surface $\left(41 / 1,000 \mathrm{~m}^{3}\right)$ when compared with bottom $\left(11 / 1,000 \mathrm{~m}^{3}\right)$ and mid-depth $\left(9 / 1,000 \mathrm{~m}^{3}\right)$. The density difference between the latter two depth strata was nonsignificant.

When depth strata pooled were pooled over all transects and stations, percent occurrence of macrophytes was similar at surface $(48 \%)$, mid-depth $(44 \%)$, and bottom ( $54 \%$ ) depth strata. All three depth strata were dominated by the same macrophytes; Nitella, Potamogeton, and Elodea. Surface samples were characterized by only five taxa; whereas, remaining depth strata each had nine plant taxa. 


\section{Station Density Comparisons Within Transects}

Frechette Point--

Comparison of total benthic drift density during the winter indicated stations $1-4$ had significantly greater abundances of drifting benthos $\left(535-1,216 / 1,000 \mathrm{~m}^{3}\right)$ than did stations 5-7 (20$38 / 1,000 \mathrm{~m}^{3}$ ) (Table 15). In addition, greatest diversity was present at stations 2-4 (14-2l taxa), with remaining stations having $\leq 8$ taxa present. Although densities of Hydra did not strongly differ across stations $\left(4-212 / 1,000 \mathrm{~m}^{3}\right)$, it made up a considerably greater portion of the drifting benthos at stations 5 and $6(65-70 \%)$ than at remaining stations $(\leq 20 \%)$. At these latter stations, Chironomidae was the dominant form (83-99\%). Mysis relicta occurred in similar densities at all stations (0-7/ $1,000 \mathrm{~m}^{3}$ ) but was generally greatest in abundance at stations 14 .

There were no significant station differences for total benthic drift density during summer. However, all major components of the benthic drift except Ephemeroptera and Hydracarina had maximal densities at stations 1-3. The proportion of total benthic drift density excluding Hydra decreased from station $1(75 \%)$ to station $7(32 \%)$. Excluding station I (20 taxa), the number of taxa collected at each station during the summer declined in the same manner that total benthic drift density without Hydra did, with greatest diversity at station 2 ( 33 taxa) and least at station 7 (21 taxa).

Greatest numbers of $\mathrm{f}$ ish larvae were captured at stations 4, 5 , and $6\left(85-139 / 1,000 \mathrm{~m}^{3}\right)$, with those at station 5 being the highest (Table 15). Remaining station density estimates ranged from $41 / 1,000 \mathrm{~m}^{3}$ to $56 / 1,000 \mathrm{~m}^{3}$. However, there were no significant differences in larval fish drift densities among stations.

The only macrophyte occurring at all stations, regardless of season, was Potamogeton (Table 15). During winter, Elodea occurred only at stations $1-4$, and stations 5-7 had only Potamogeton present (except station 7 which had two samples with Chara). During summer, Potamogeton, Elodea, and Nitella occurred at nearly all stations. Remaining plant taxa occurred randomly among stations, except Bryum which was notably frequent at station 7. During both seasons, station 4 had the highest diversity of macrophytes.

Lake Nicolet--

No significant differences in total benthic drift density among stations were evident during either winter or summer. In 


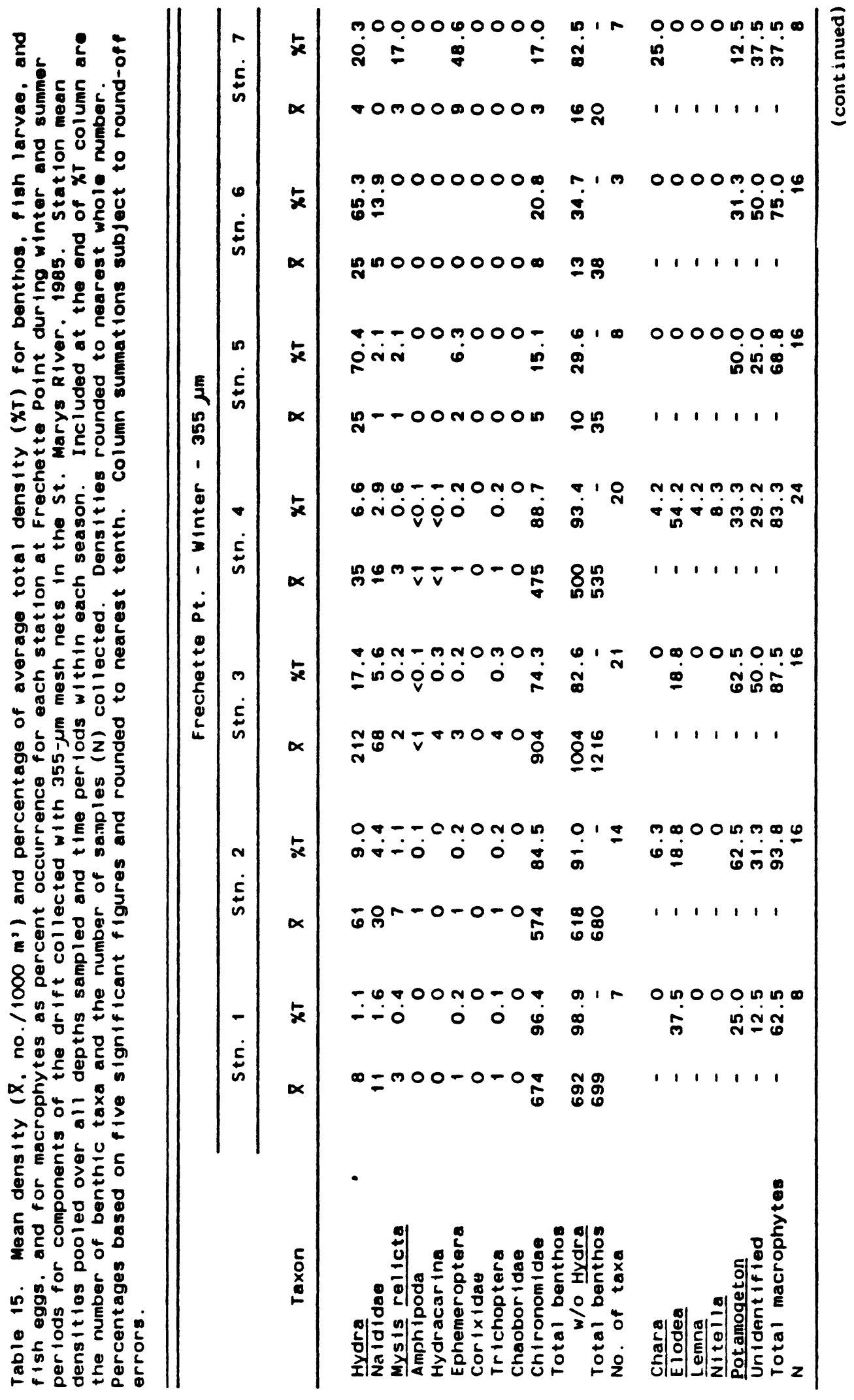




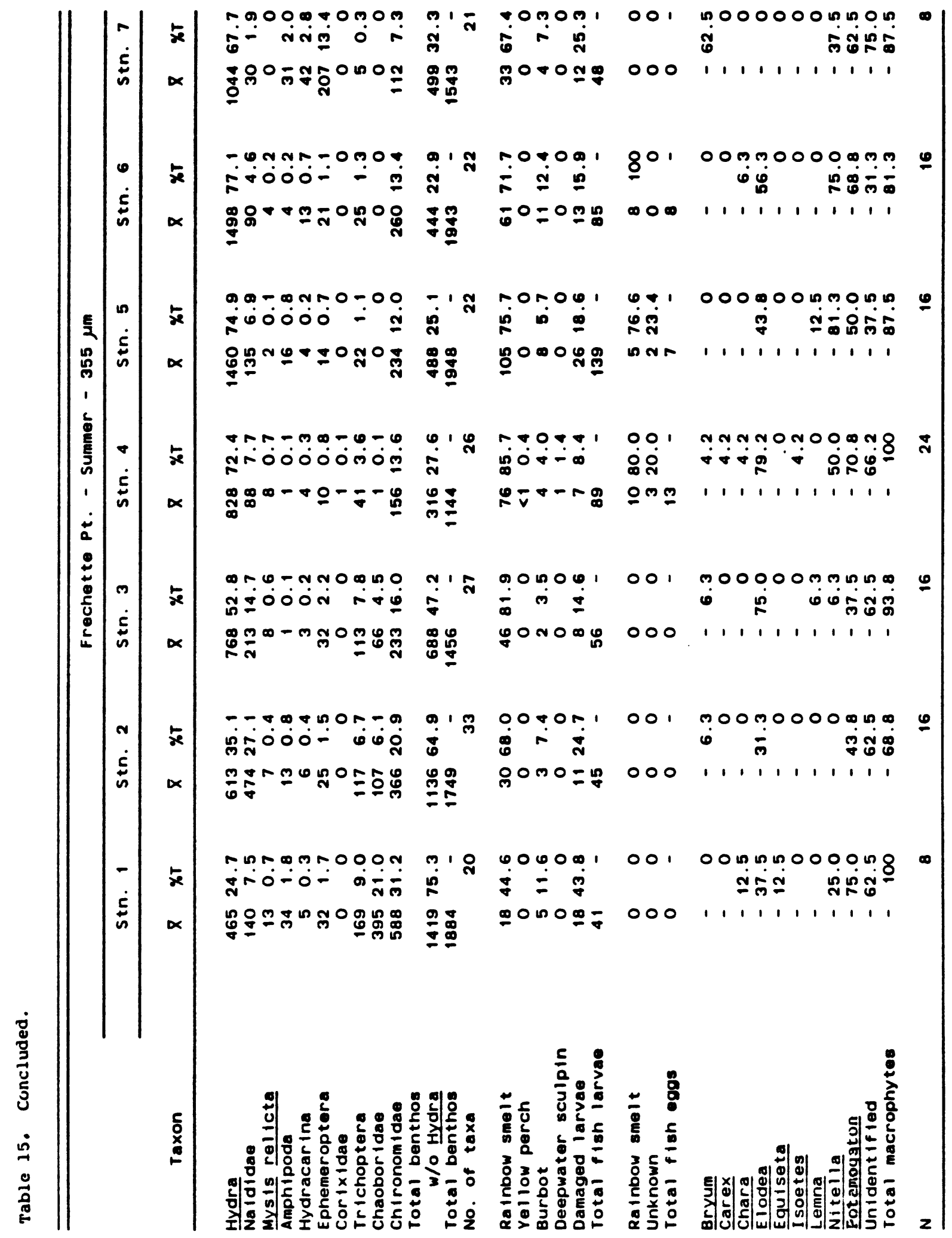


addition, at most stations the number of taxa collected was similar (2-8) (Table 16). During winter, the most notable trends among major components of the benthic drift were: (1) at stations 1-4, mysids were most numerous, and (2) at station 9, Chironomidae and Ephemeroptera were the only taxa encountered and they were very abundant relative to other stations.

During summer, benthic drift at stations $1-3$ and $8-9$ was largely Ephemeroptera (36-63\%), with the remaining, more mid-lake stations 4-7 dominated by Chironomidae (51-70\%). Remaining major benthic drift components were most abundant at depths $\leq 3 \mathrm{~m}$.

Even though very high larval fish drift abundances were encountered at station 5 in the central portion of Lake Nicolet (up to $15,600 / 1,000 \mathrm{~m}^{3}$, see Appendixes 87 and 88 ), average station density estimates for stations $1-8$ ranging from $101 / 1,000$ $\mathrm{m}^{3}$ to $347 / 1,000 \mathrm{~m}^{3}$ were non-significant. However, all estimates of larval fish drift were significantly greater than at station 9 which averaged only $21 / 1,000 \mathrm{~m}^{3}$.

During winter at Lake Nicolet, macrophytes were most frequently encountered at stations 3 and 4 . None or very few plants occurred at stations 2 and 7-9. The dominant plant occurring in Lake Nicolet drift samples was Nitella. During summer, macrophytes were collected at stations 1-4 and 6, wherein Nitella was dominant at all except station 1 where Utricularia occurred in equal frequency with Nitella (13\%) (Table 16).

\section{Point aux Frenes--}

During winter total benthic drift density was dominated by chironomidae; there were no significant station differences. However, during summer, the two stations nearest shore ( 1 and 7 ) had significantly greater total benthic drift density $(1,712)$ $1,000 \mathrm{~m}^{3}$ and $1,328 / 1,000 \mathrm{~m}^{3}$, respectively) than did the navigation channel, station $4\left(88 / 1,000 \mathrm{~m}^{3}\right)$, but these two stations were not significantly different from stations $2-3$ and 5-6 (206-582/1,000 $\left.\mathrm{m}^{3}\right)$ (Table 17). The drifting benthos at all stations was dominated by Ephemeroptera, Hydracarina, Corixidae, Chironomidae, and Amphipoda. The number of benthic taxa collected at each station was similar (8-1l) except at station 7 where 16 taxa were collected.

No $f$ ish larvae were collected at stations 1,2 , and 6 . Maximum numbers were collected in the drift at stations 4 (37) $\left.1,000 \mathrm{~m}^{3}\right)$ and $7\left(28 / 1,000 \mathrm{~m}^{3}\right)$. Drifting $\mathrm{fish}$ larvae estimates ranged from $2 / 1,000 \mathrm{~m}^{3}$ at station 3 to $10 / 1,000 \mathrm{~m}^{3}$ at station 5 . The latter two stations had significantly fewer fish larvae than did station 4 , but neither was significantly different from 


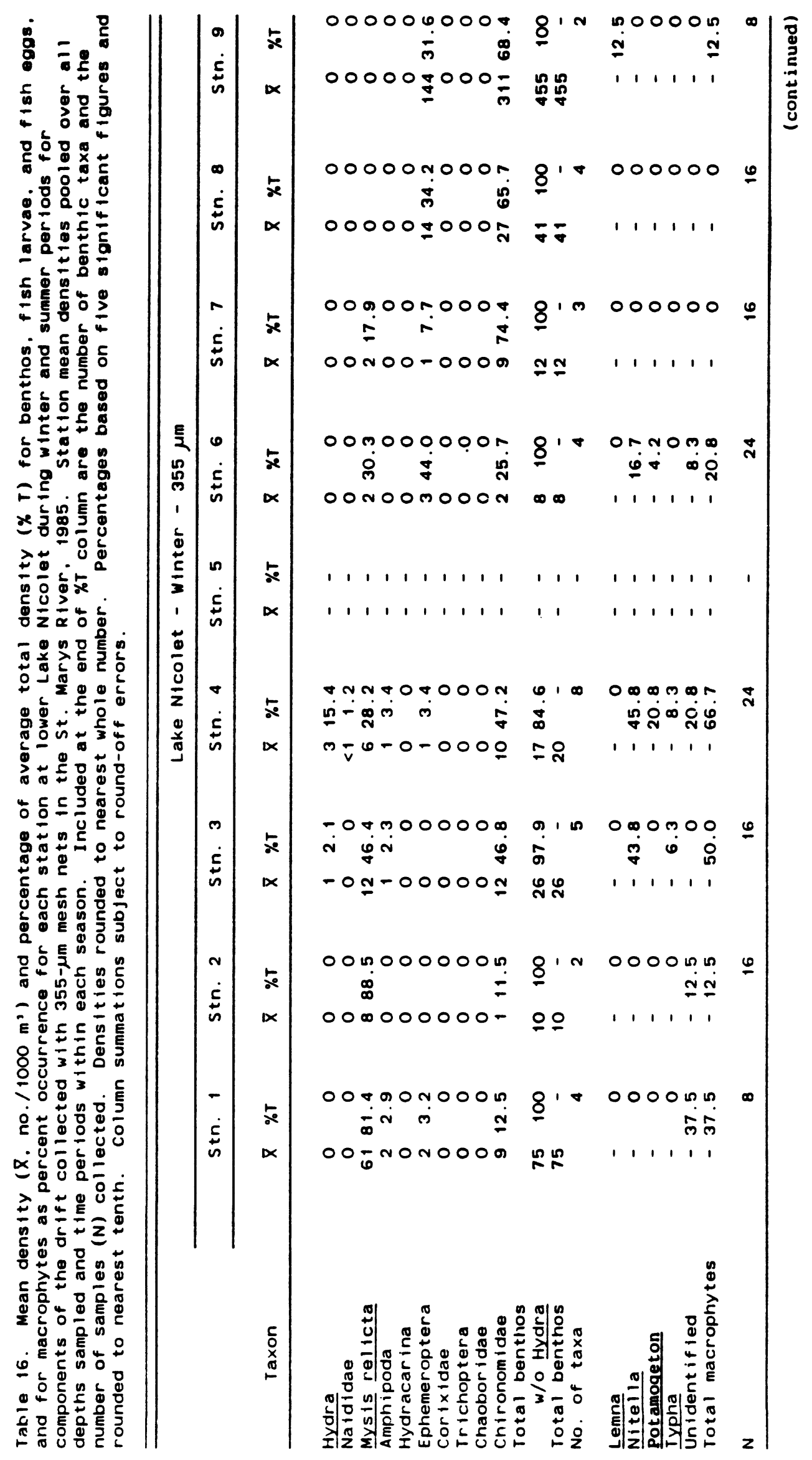




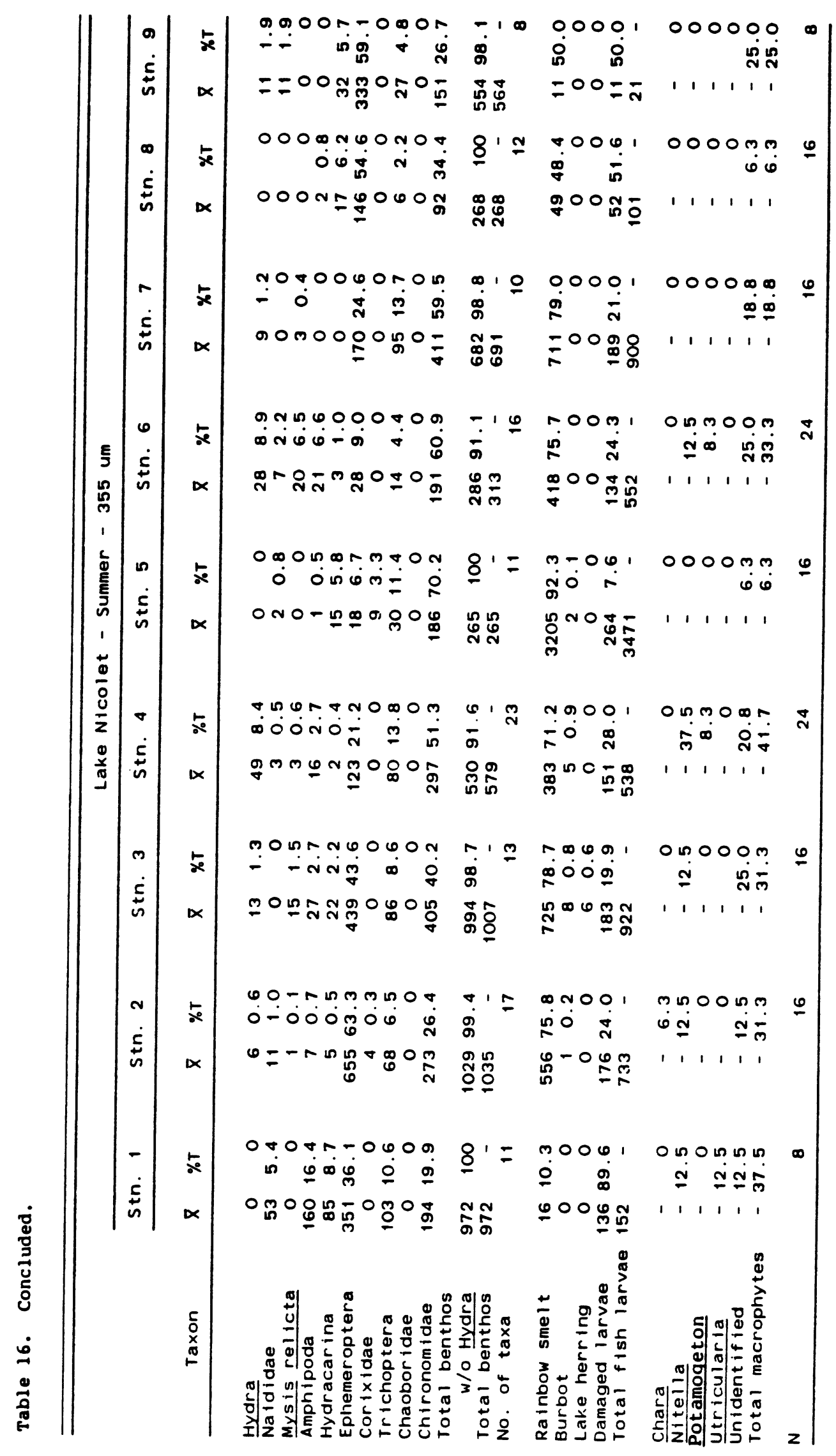




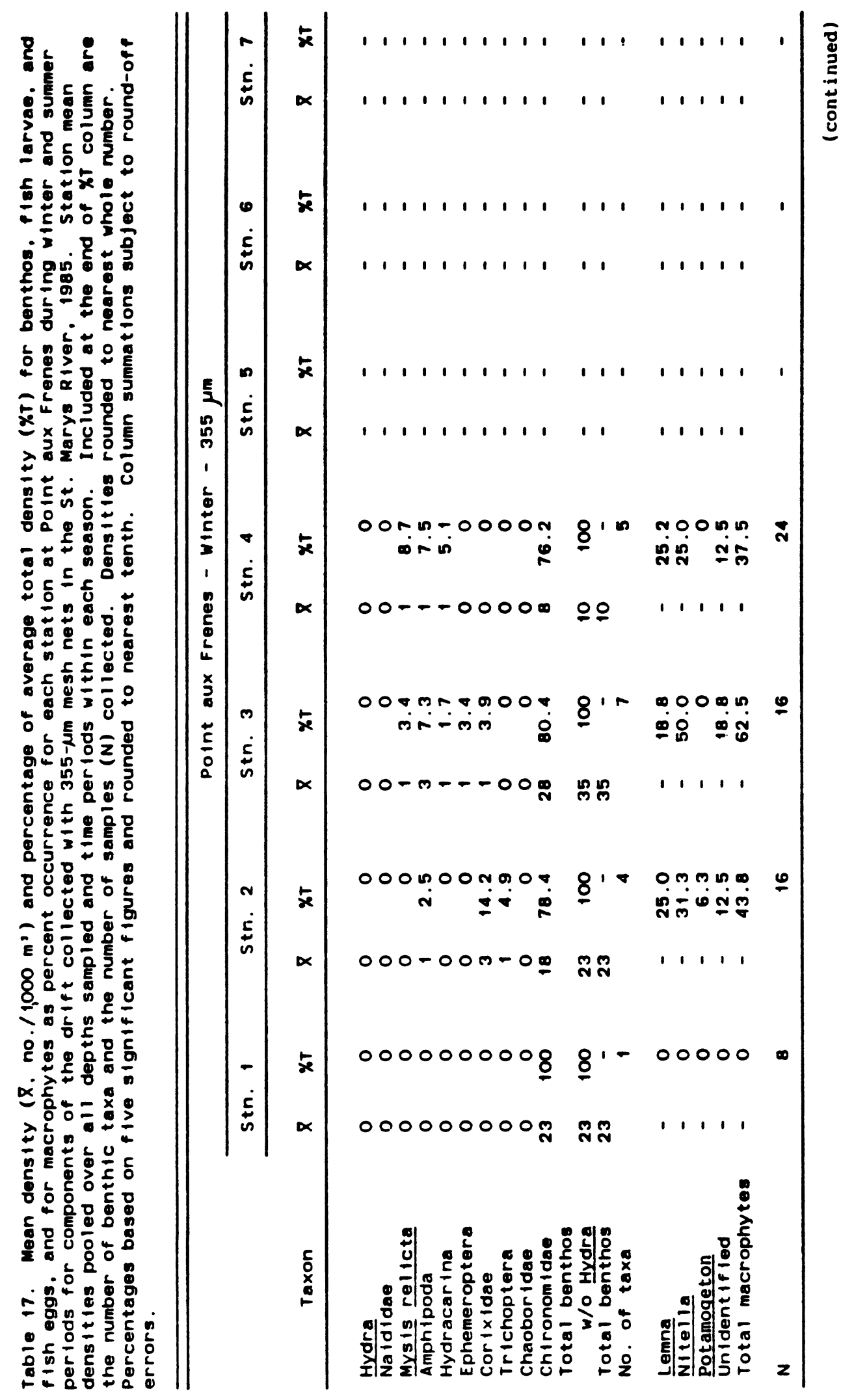




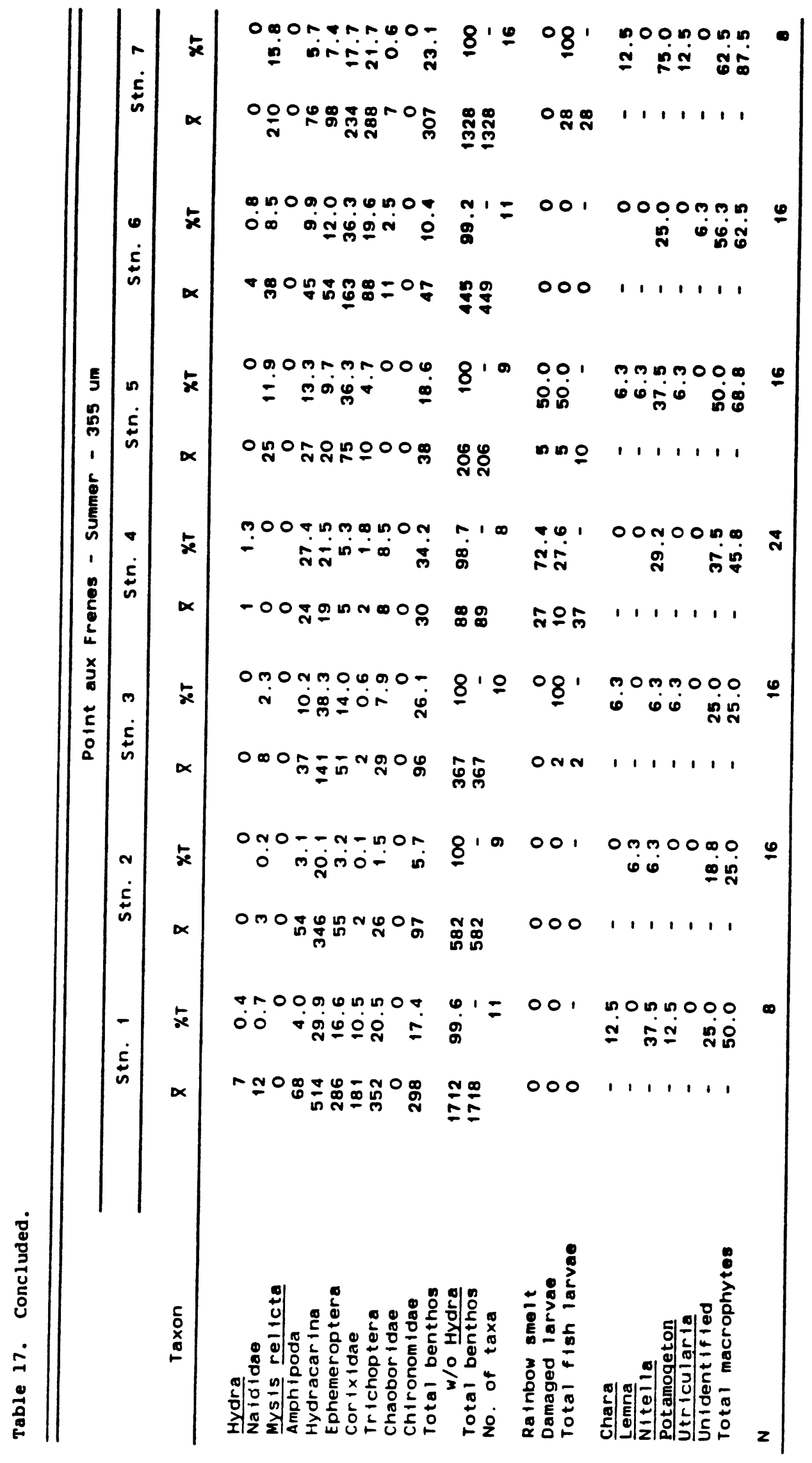


station 7. Density differences between stations 4 and 7 were non-significant.

Nitella and Lemna were the most frequently occurring macrophytes at stations 2-4 during winter at Point aux Frenes. No plants were collected at station 1 . During summer, plants were collected in greatest frequency at stations 7-9. However, regardless of how frequently macrophytes were encountered at each station, Nitella occurred most of ten or shared equal dominance with other plants at all stations.

\section{Weather Effects on Drift Densities}

Lake Nicolet--

During the two summer day-periods at Lake Nicolet, two very different weather conditions were encountered. On Day 1 ( 6 June 1985) at stations 1-4, weather conditions changed from a short period of calm winds and rain to sunny and windy resulting in 0.3-0.6-m waves. On Day 2 ( 7 June 1985), the river in the vicinity of stations $1-4$ had no waves and was essentially calm. Day 1 current velocities at the shallower stations (1-3) averaged $27 \mathrm{~cm} / \mathrm{s}$ and were considerably greater than those measured on Day 2 under calm conditions $(8 \mathrm{~cm} / \mathrm{s})$. Little variation in current velocity was noted at station $4(21 \mathrm{vs} .20 \mathrm{~cm} / \mathrm{s})$. No ships passed downbound on the windy day, however, the Canadian Olympic and the Kupa passed downbound on the calm day while nets were set at station 1 .

Based on estimates pooled over stations 1-4, samples from the windy day had significantly greater numbers of Chironomidae $\left(42 / 1,000 \mathrm{~m}^{3}\right.$ vs. $\left.19 / 1,000 \mathrm{~m}^{3}\right)$ and total benthic drift $(78 / 1,000$ $\mathrm{m}^{3}$ vs. $41 / 1,000 \mathrm{~m}^{3}$ ) when compared with samples from the calm day (Table 18). While no significant density differences were noted for other major benthic drift components or for total $f$ ish larval drift, for nearly all, greatest densities occurred on the windy day.

\section{Point aux Frenes--}

A similar wind event occurred at Point aux Frenes. On Day 1 (11 June 1985) wave height was $0.3 \mathrm{~m}$ to $0.6 \mathrm{~m}$ at stations 1-3, but on Day 2 ( 12 June 1985) it was reduced to $<0.3 \mathrm{~m}$. Only the comparison for Chironomidae was significant, with greater densities observed on the windy day $\left(29 / 1,000 \mathrm{~m}^{3}\right)$ when compared with the calm day $\left(7 / 1,000 \mathrm{~m}^{3}\right)$. However, in many comparisons, though non-significant, drift densities were greatest on the windy day (Table 18). No fish larvae were collected at stations 1-3 during the day. 
Table 18. Mean density $\left(\bar{x}\right.$, no. $\left./ 1,000 \mathrm{~m}^{3}\right)$ and percentage of average total density $(\& \mathrm{~T})$ for benthos, $\mathrm{f}$ ish larvae, and $f$ ish eggs pooled over stations 1-4 at Lake $\mathrm{Nicolet}$ and over stations $1-3$ at Point Aux Frenes during the summer under conditions of calm and windy weather for components of the drift collected with 355-um mesh nets in the St. Marys River, 1985. Included at the end of $\$ T$ column are the number of benthic taxa and the number of samples $(N)$ collected. Densities rounded to nearest whole number. Percentages based on $f$ ive significant figures and rounded to nearest tenth. Column summations subject to round-off errors.

\begin{tabular}{|c|c|c|c|c|c|c|c|c|}
\hline \multirow[b]{3}{*}{ Taxon } & \multicolumn{4}{|c|}{ Lake Nicolet } & \multirow{2}{*}{\multicolumn{4}{|c|}{$\frac{\text { Point aux Frenes }}{\text { Calm Windy }}$}} \\
\hline & \multicolumn{2}{|c|}{ Calm } & \multicolumn{2}{|c|}{ windy } & & & & \\
\hline & $\overline{\mathrm{x}}$ & $8 T$ & $\overline{\mathrm{x}}$ & \&T & $\overline{\mathrm{X}}$ & $8 T$ & $\overline{\mathbf{x}}$ & $\& \mathrm{~T}$ \\
\hline $\begin{array}{l}\text { Hydra } \\
\text { Naididae } \\
\text { Mysis relicta } \\
\text { Amphipoda } \\
\text { Hydracarina } \\
\text { Ephemeroptera } \\
\text { Corixidae } \\
\text { Trichoptera } \\
\text { Chaoboridae } \\
\text { Chironomidae } \\
\text { Total benthos }\end{array}$ & $\begin{array}{l}8 \\
0 \\
0 \\
3 \\
5 \\
3 \\
0 \\
9 \\
0 \\
19\end{array}$ & $\begin{array}{r}15.6 \\
0 \\
0 \\
5.4 \\
10.6 \\
5.3 \\
0 \\
18.8 \\
0 \\
39.0\end{array}$ & $\begin{array}{r}48 \\
16 \\
1 \\
6 \\
4 \\
0 \\
0 \\
3 \\
0 \\
42\end{array}$ & $\begin{array}{r}38.0 \\
13.0 \\
1.2 \\
4.4 \\
2.9 \\
0 \\
0 \\
2.0 \\
0 \\
33.8\end{array}$ & $\begin{array}{r}0 \\
5 \\
0 \\
0 \\
38 \\
0 \\
15 \\
5 \\
0 \\
7\end{array}$ & $\begin{array}{r}0 \\
7.0 \\
0 \\
0 \\
54.4 \\
0 \\
21.1 \\
7.0 \\
0 \\
10.5\end{array}$ & $\begin{array}{r}5 \\
3 \\
0 \\
0 \\
63 \\
3 \\
3 \\
3 \\
0 \\
29\end{array}$ & $\begin{array}{r}4.8 \\
2.4 \\
0 \\
0 \\
58.3 \\
2.4 \\
2.7 \\
2.4 \\
0 \\
26.9\end{array}$ \\
\hline $\begin{array}{l}\text { w/o Hydra } \\
\text { Total beenthos } \\
\text { No. of taxa }\end{array}$ & $\begin{array}{l}41 \\
48\end{array}$ & $\begin{array}{r}84.4 \\
\frac{8}{8}\end{array}$ & $\begin{array}{r}78 \\
125\end{array}$ & $\begin{array}{r}62.0 \\
10\end{array}$ & $\begin{array}{l}69 \\
69\end{array}$ & $\begin{array}{r}100 \\
\frac{5}{5}\end{array}$ & $\begin{array}{l}102 \\
108\end{array}$ & $\frac{95.1}{7}$ \\
\hline $\begin{array}{l}\text { Rainbow smelt } \\
\text { Burbot } \\
\text { Damaged larvae } \\
\text { Total fish larvae }\end{array}$ & $\begin{array}{r}227 \\
0 \\
74 \\
301\end{array}$ & $\begin{array}{r}75.3 \\
0 \\
24.7 \\
-\end{array}$ & $\begin{array}{r}253 \\
11 \\
63 \\
327\end{array}$ & $\begin{array}{r}77.5 \\
3.2 \\
19.3 \\
-\end{array}$ & $\begin{array}{l}0 \\
0 \\
0 \\
0\end{array}$ & $\begin{array}{l}0 \\
0 \\
0 \\
-\end{array}$ & $\begin{array}{l}0 \\
0 \\
0 \\
0\end{array}$ & $\begin{array}{l}0 \\
0 \\
0 \\
-\end{array}$ \\
\hline $\mathrm{N}$ & & 16 & & 16 & & 10 & & 10 \\
\hline
\end{tabular}


Frechette Point--

Weather comparisons at Frechette Point were confounded by diel differences, because weather conditions on Days 1 and 2 were windy (0.3- to $0.6-\mathrm{m}$ waves), while night weather conditions were calm with no waves. As previously noted total benthic drift density estimates pooled over all siations indicated the windy day densities $\left(2,488 / 1,000 \mathrm{~m}^{3}\right)$ significantly exceeded calm night densities $\left(750 / 1,000 \mathrm{~m}^{3}\right)$ by a factor of 3.32 . The number of $\mathrm{f}$ ish larvae caught during the day $\left(88 / 1,000 \mathrm{~m}^{3}\right)$ exceeded, but not significantly, the number retained at night $\left(67 / 1,000 \mathrm{~m}^{3}\right)$ by a factor of 1.31. Additionally, the tug, Canonie, and a barge it was guiding passed upbound during the day. At night the Roger M. Keys, Belle River, St. Clair, and Fred E. White Jr. passed downbound while a variety of station nets were set. Regardless of the higher number of ship passages during the night, daytime densities for both benthos and $f$ ish larvae were greater than night densities.

\section{Ship Passage Drift Studies}

Introduction--

Upbound and downbound ship passage studies were conducted on 10 June 1985 during daylight. Weather for both studies was sunny and windy. Wave height at Frechette Point was $0.3 \mathrm{~m}$ to $1.0 \mathrm{~m}$, with wind speeds ranging from $26 \mathrm{~km} / \mathrm{h}$ to $44 \mathrm{~km} / \mathrm{h}$. The upbound portion of the study was based on the 1031 EDT passage of the Comeaudoc (length $=223 \mathrm{~m}$, width $=23 \mathrm{~m}$, draft $=4.6 \mathrm{~m}$ ). The speed of the Comeaudoc was $13.2 \mathrm{~km} / \mathrm{h}$. The downbound portion of the study was based on the 1437 EDT passage of the V.W. Scully (length $=223 \mathrm{~m}$, width $=23 \mathrm{~m}$, draft $=7.6 \mathrm{~m}$ ). The speed of the V.W. Scully was $13.5 \mathrm{~km} / \mathrm{h}$. The passage of both vessels was

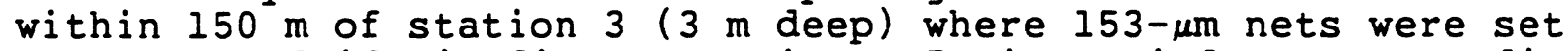
to collect drift in five approximate 5-min periods corresponding to Before-, During-, After 1- $(+5 \mathrm{~min})$, After 2- $(+10 \mathrm{~min})$, and After $3-(+15 \mathrm{~min})$ ship passage periods.

Passage of the upbound ship had a much greater visible effect on the physical appearance of the sampling device than did the downbound vessel. When placed in the water with nets attached, the main support rod of the sample device was normally deflected 10-15 from the vertical at Frechette Point. However, during the 2-min period when the Comeaudoc passed nearest the nets, the main support rod was displaced $20-25$ from vertical. Additionally, mid-depth current velocity increased from $41-43 \mathrm{~cm} /$ $s$ to $54-56 \mathrm{~cm} / \mathrm{s}$. In the following minute, current velocity decreased to $44 \mathrm{~cm} / \mathrm{s}$, and the main support rod returned to its normal deflection in the current. 
With passage of the downbound vessel, there was no observable deflection of the main support rod from its normal position in the $8 \mathrm{~min}$ representing the During-ship-passage period. During this $8-\mathrm{min}$ period, in the $5 \mathrm{~min}$ prior to the V.W. Scully's passage, mid-depth current velocity ranged from 46 $\mathrm{cm} / \mathrm{s}$ to $54 \mathrm{~cm} / \mathrm{s}$. However, in the 2-min period during which the vessel passed closest to the nets, current velocity decreased to $43 \mathrm{~cm} / \mathrm{s}$ and $41 \mathrm{~cm} / \mathrm{s}$, respectively. In the ensuing minute just after immediate passage, current velocity decreased further to 40 $\mathrm{cm} / \mathrm{s}$.

Estimates of downbound benthic and ichthyoplankton drift catches required correction, because at least one of the two replicate nets located at the bottom during the second 5-min period after passage (After 2) of the downbound V.W. Scully apparently tangled around a portion of the sample device and did not sample properly. As one replicate had no benthos and another had only a low number relative to the mid-depth net catch, estimates of the drift near bottom in the After 2 period were not considered in statistical comparisons. However, estimates from these two nets do appear in density averages in tables where they may be part of a pooled average (Tables 19 and 29).

Comparisons of Upbound and Downbound Pooled Drift--

When comparing upbound $(\mathrm{N}=20)$ and downbound $(\mathrm{N}=18)$ mean drift density pooled over all respective samples, drift was significantly greater for Naididae $\left(4,623 / 1,000 \mathrm{~m}^{3}\right.$ vs. 1,292/ $\left.1,000 \mathrm{~m}^{3}\right)$, Chironomidae $\left(2,329 / 1,000 \mathrm{~m}^{3}\right.$ vs. $\left.1,536 / 1,000 \mathrm{~m}^{3}\right)$, and total benthic drift $\left(9,608 / 1,000 \mathrm{~m}^{3}\right.$ vs. $\left.5,416 / 1,000 \mathrm{~m}^{3}\right)$ in upbound samples (Tables 19-20). Upbound and downbound density estimates for all other major components of the benthic drift and for larval fish drift were statistically non-significant. While Hydra, Naididae, and Chironomidae were the dominant benthic taxa in all cases, Naididae was the most numerous of the 53 benthic taxa (Table l) collected in the upbound vessel study (48\% of total benthic drift), and Hydra was predominant among the 11 taxa collected in the downbound vessel study (45\%). In both studies, rainbow smelt was the dominant larval $f$ ish, making up $>90 \%$ of total $f$ ish larvae. The only other larval fish encountered was burbot which made up $<3 \%$ of total fish larvae.

Comparisons of Mid-depth and Bottom Pooled Drift in the Upbound Study--

All comparisons of benthic drift densities pooled over middepth and bottom depth strata $(N=10)$ in the upbound vessel study were non-significant except for Naididae. Naidid drift density was significantly greater at bottom $\left(6,319 / 1,000 \mathrm{~m}^{3}\right)$ than 

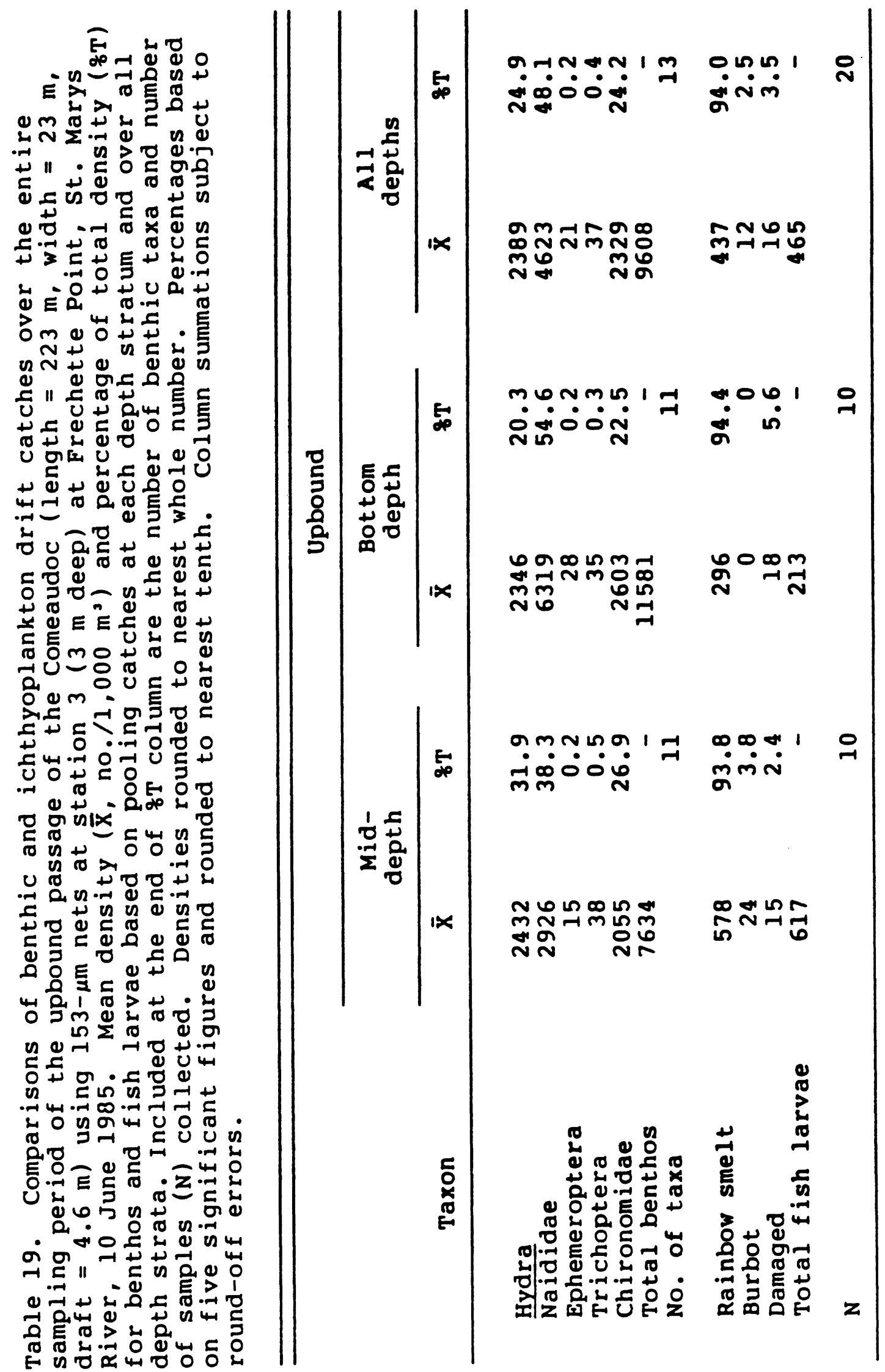


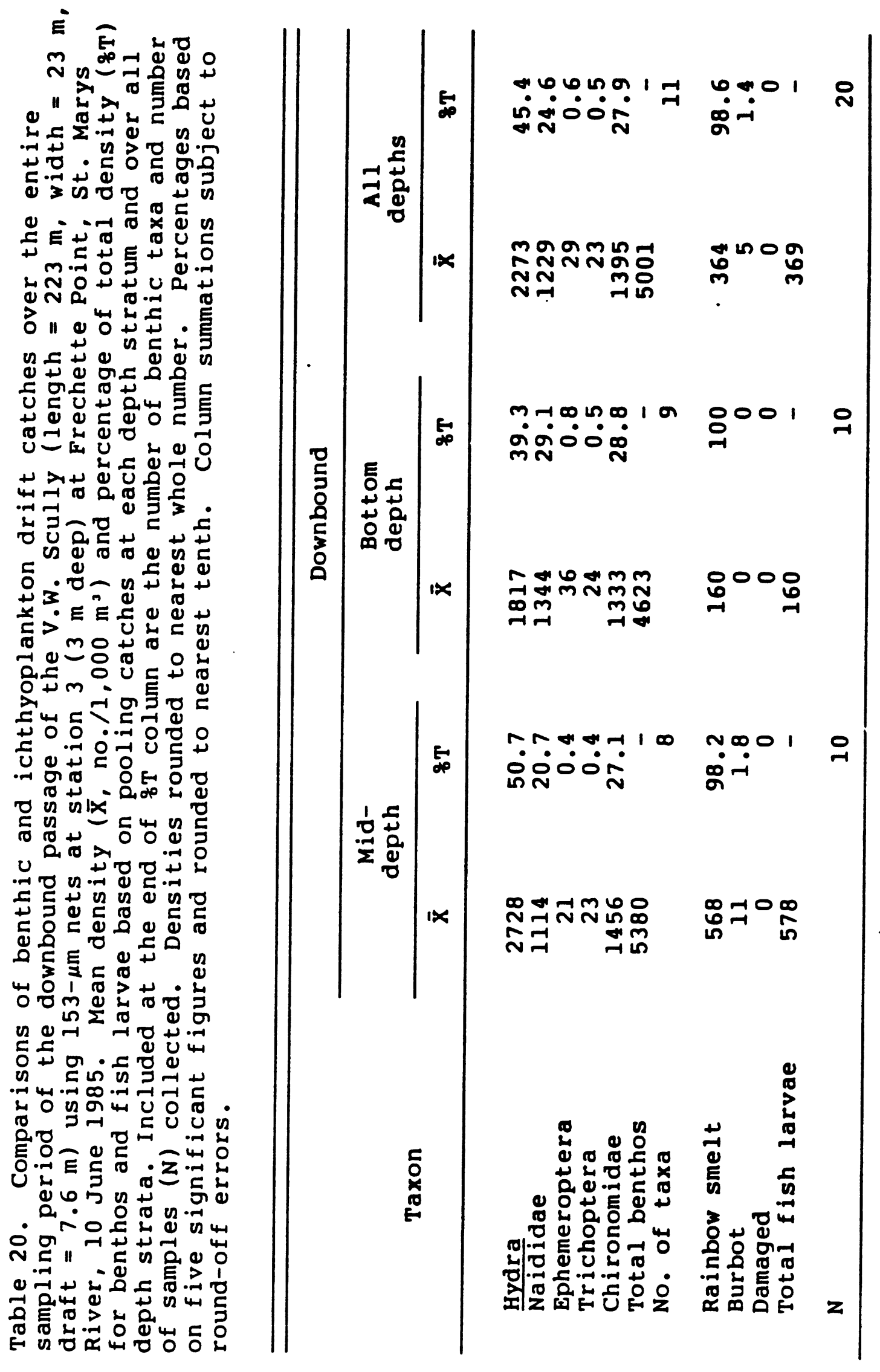


at mid-depth $\left(2,926 / 1,000 \mathrm{~m}^{3}\right)$. Total larval $\mathrm{fish}$ drift density was significantly higher at mid-depth $\left(617 / 1,000 \mathrm{~m}^{3}\right)$ when compared with bottom $\left(313 / 1,000 \mathrm{~m}^{3}\right)$.

Comparisons of Mid-depth and Bottom Pooled Drift in the Downbound Study--

Data on drift from mid-depth and bottom were pooled in the same manner as was done for the upbound study. We found no statistically significant drift density differences between mid- $(\mathrm{N}=10)$ and bottom $(\mathrm{N}=8)$ depth strata for major benthic components of the drift. However, as in the upbound vessel study, total larval fish drift density was significantly greater at mid-depth $\left(578 / 1,000 \mathrm{~m}^{3}\right)$ when compared with the bottom strata $\left(184 / 1,000 \mathrm{~m}^{3}\right)$.

Comparisons of the Five Ship Passage Periods at Each Depth Stratum and Combined Depth Strata for Respective Upbound and Downbound Studies--

The only significant density difference among either upbound or downbound ship passage periods at mid-depth $(N=2)$, bottom ( $N$ $=2)$, and for both strata combined $(N=4)$ was for total larval fish drift density at mid-depth in the upbound study. In this comparison, total larval $f$ ish drift density in the After l period $\left(369 / 1,000 \mathrm{~m}^{3}\right)$ was significantly lower than in all remaining periods for which density differences were non-significant (517$1,108 / 1,000 \mathrm{~m}^{3}$ ) (Tables 21-25).

While density trends between depth strata and between the two studies were apparent, there were no clear trends from one 5min period to another within the upbound or downbound vessel studies. Total benthic drift density in the upbound vessel study was consistently greater near bottom when compared with mid-depth during all sample periods except the After 3 period. However, in the downbound study, most mid-depth density estimates of the benthos were greater than bottom estimates. When pooling data from both depth strata, the total benthic drift density estimates during the upbound vessel passage exceeded downbound estimates during all 5-min sampling periods. When examining benthic drift density trends successively across the five sample periods, there was no consistent trend at any combination of depth strata in either study (Tables 21-25).

Similar comparisons of total larval fish drift density for upbound and downbound studies indicated mid-depth densities exceeded bottom estimates during each 5-min sampling period. When pooling data from both depth strata, total larval fish drift density in the downbound vessel study was similar during all 5- 


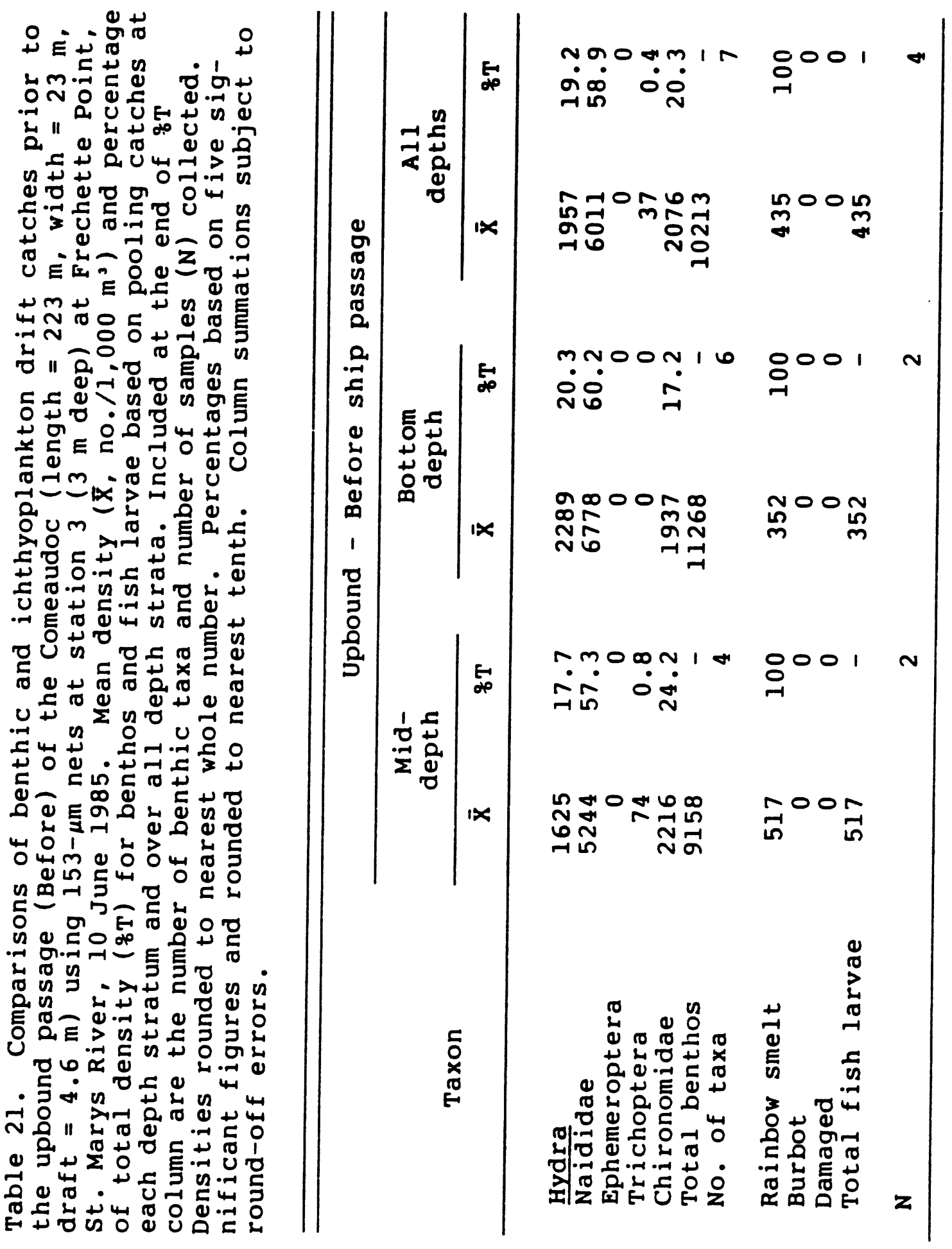



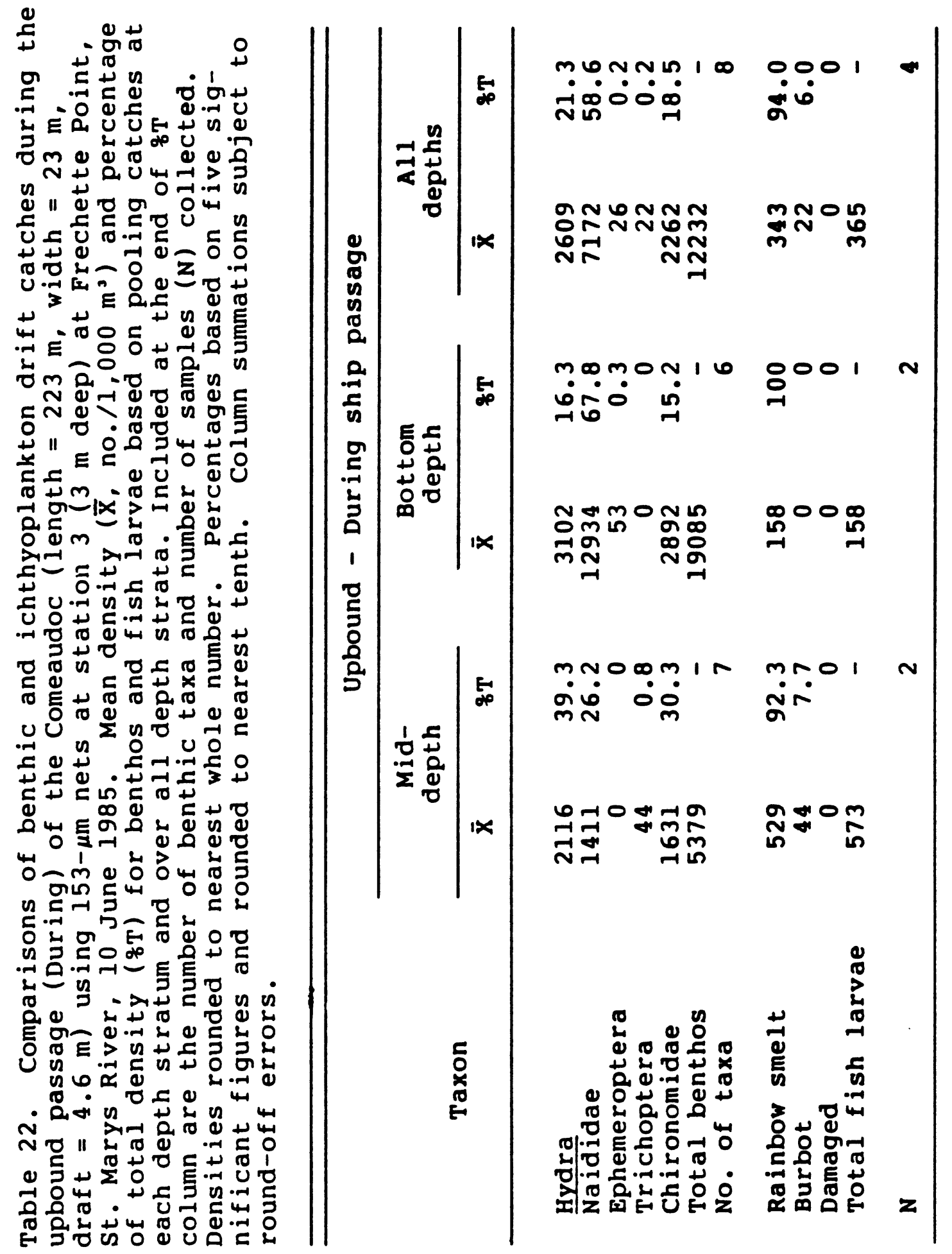


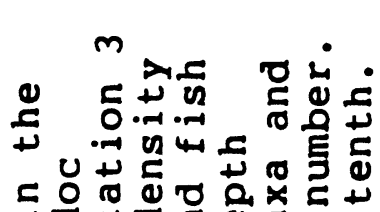

-

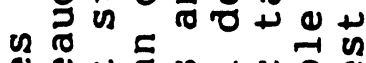

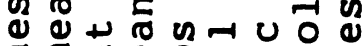

트

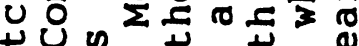

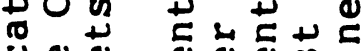

U U U م

世

4 हब थ แ त

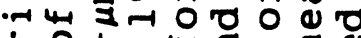

म 0 ल $\omega^{4}$ ह

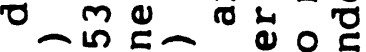

대다

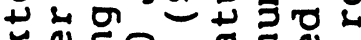

บ 1 E 0 त

E山・A

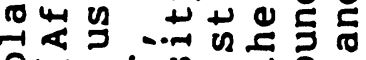

Q

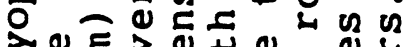

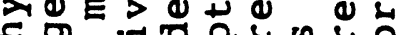

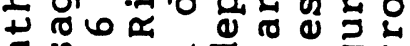

है

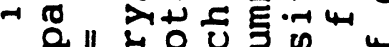

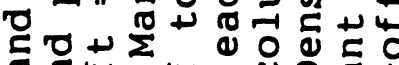

бㄷㄴ

U 0 H

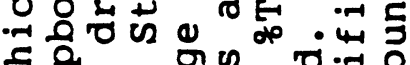

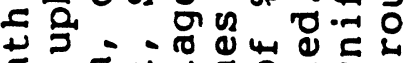

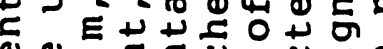

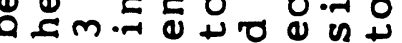
$\downarrow N 0$ U ช

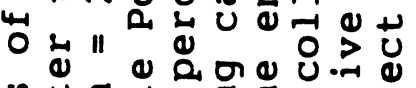
n + 대 EE 4 ᄃ

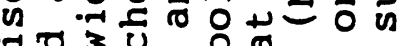
-

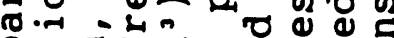

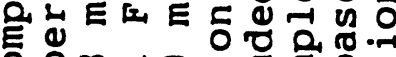
ด $0 m+0$ 의 U $N$ ⿰冫欠 드은

- E |

mi 100 \%

N

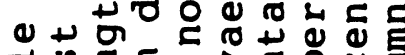
ด U ช0.4 Eu

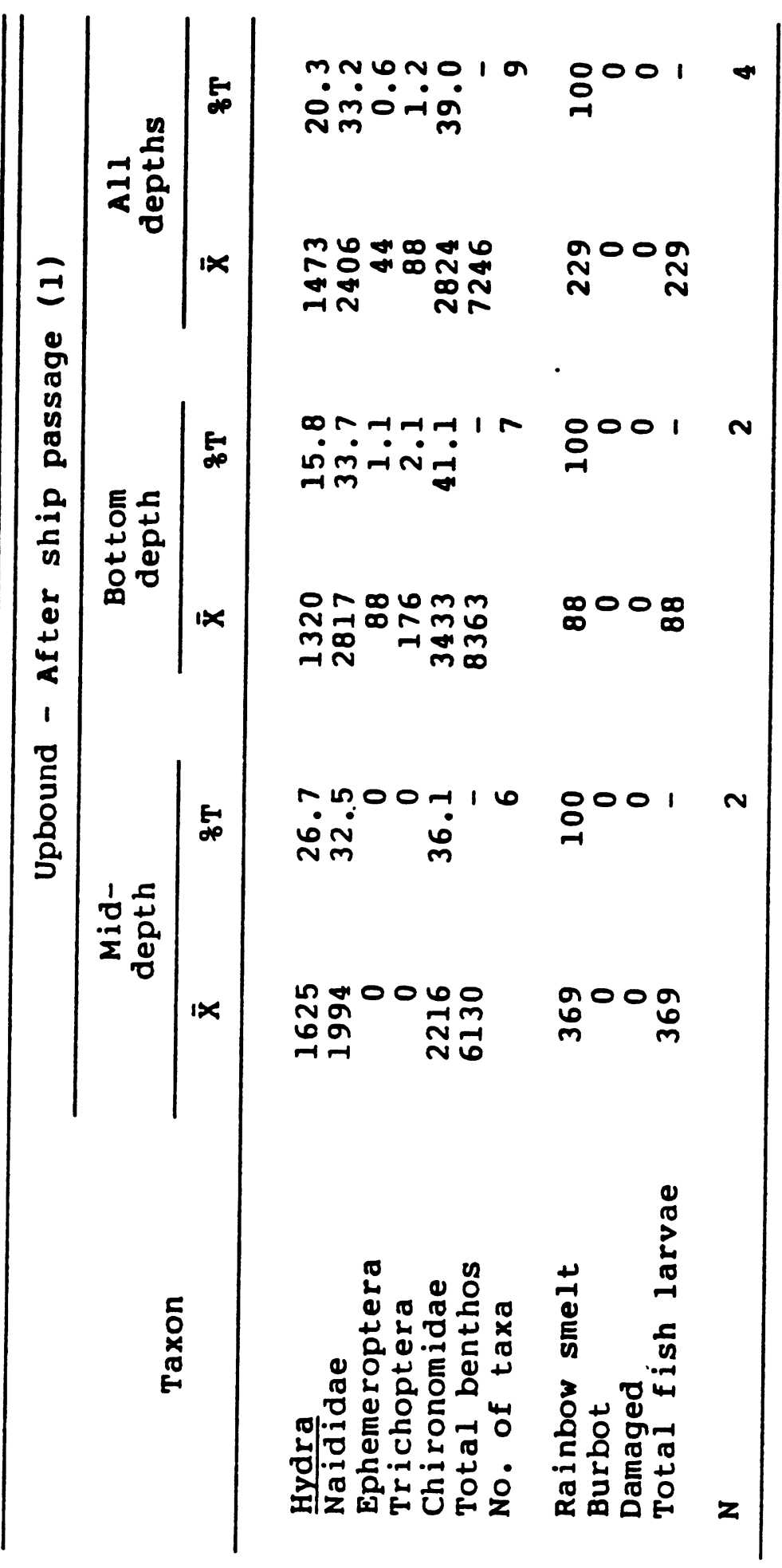




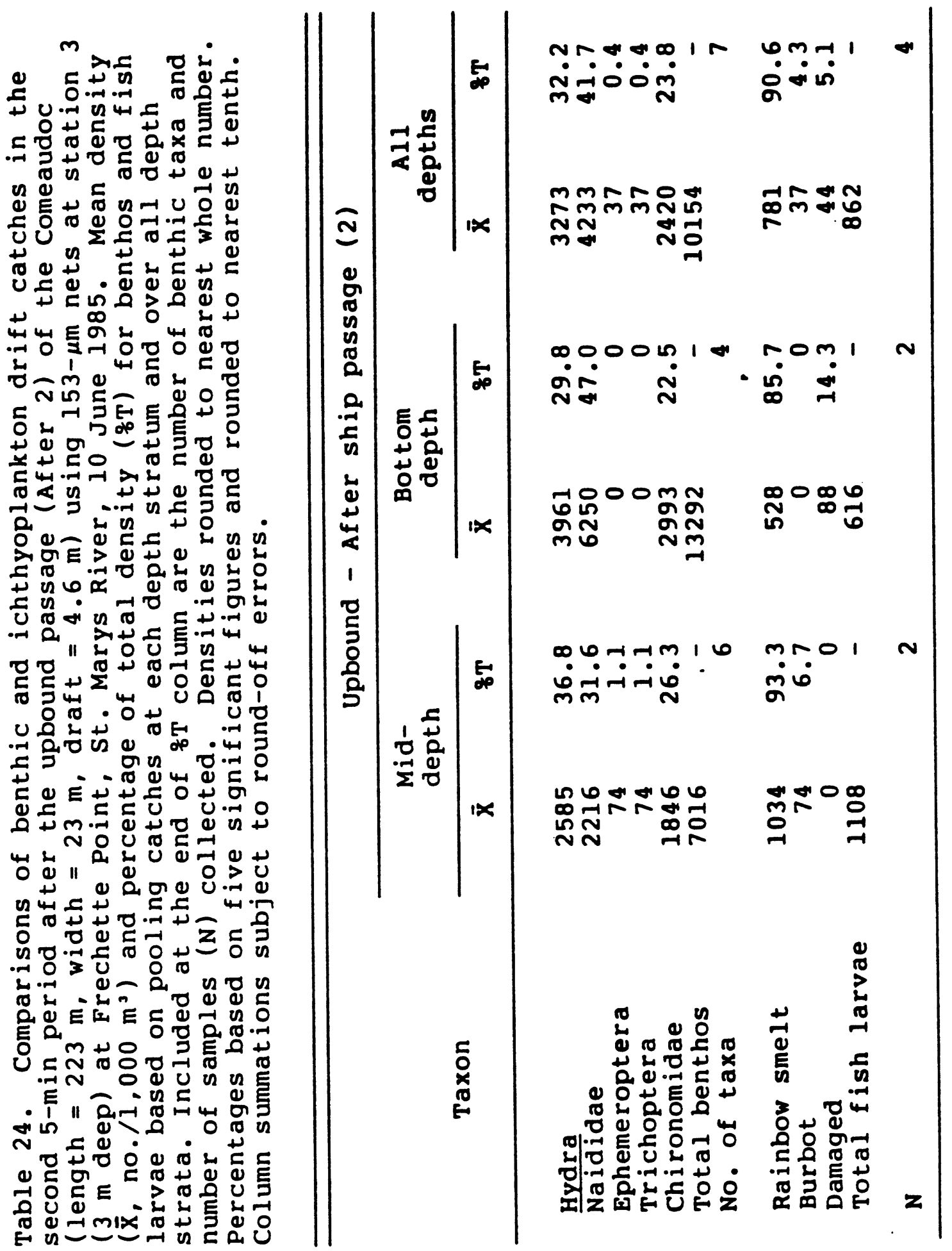


ڤ O .

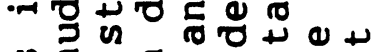

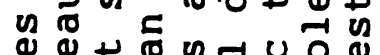

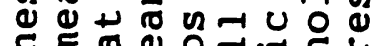

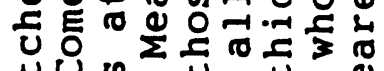

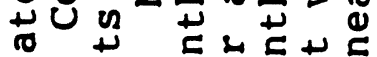

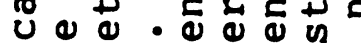

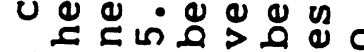
廿 대

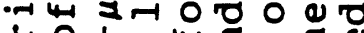
म 011 W 5 ర人

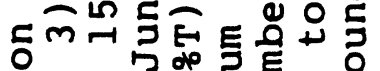
엄 万d

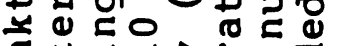

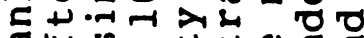

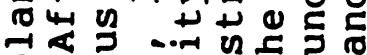
م.

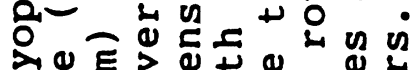

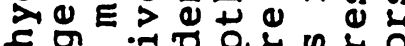

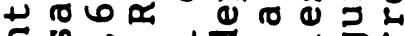

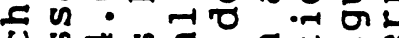

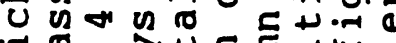
ช

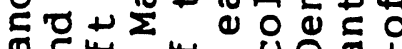
茴. U O

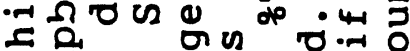

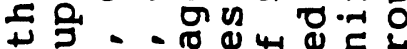

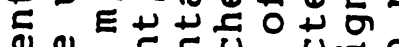

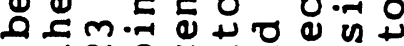

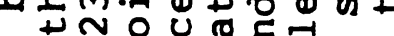
虫 U

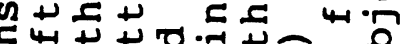

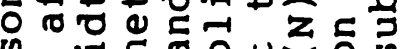
भ $\rightarrow \frac{1}{0}$ 万人 年 Tำ

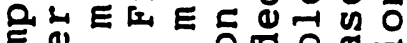
ठิ U N

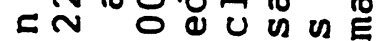
-

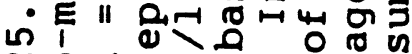
N ט ه $\rightarrow$ EE $>0$ 先 م. A

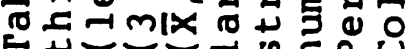

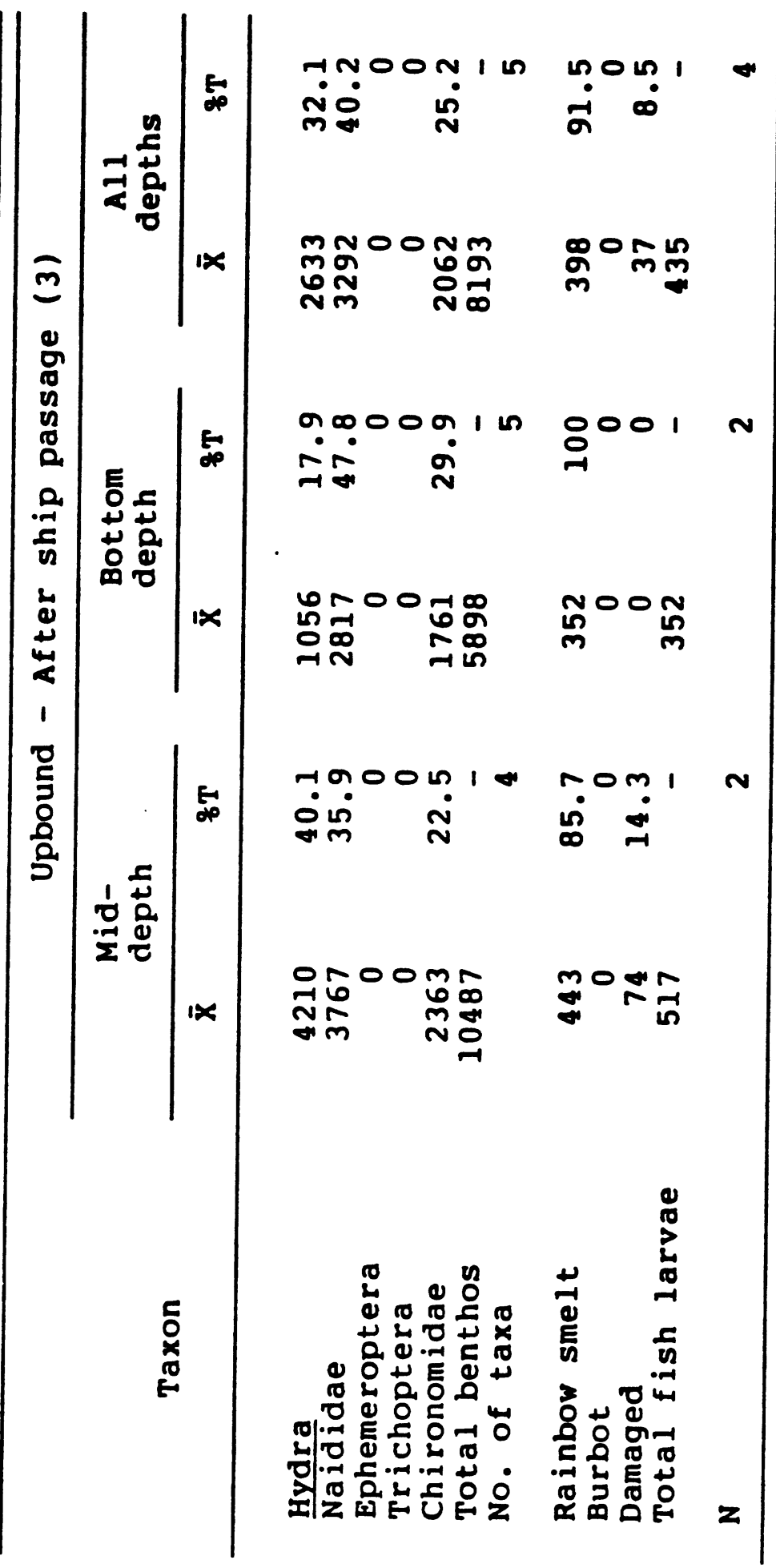


min periods (Tables 26-30). During the upbound vessel passage, there was a decline in total larval $f$ ish drift from $435 / 1,000 \mathrm{~m}^{3}$ (Before) to $365 / 1,000 \mathrm{~m}^{3}$ (During), to 229/1,000 $\mathrm{m}^{3}$ (After 1). There was a sharp increase in larval fish drift density during the After 2 period to $863 / 1,000 \mathrm{~m}^{3}$ (Tables 21-26). During the After 3 period, density was $435 / 1,000 \mathrm{~m}^{3}$ as larval $\mathrm{f}$ ish drift density returned to the level estimated during the Before period. This trend occurred at both depth strata.

\section{ZOOPLANKTON}

Zooplankton Abundance and Community Structure

Winter--

Zooplankton abundances, as determined by No. 2-mesh drift net collections, were extremely low during the winter study period (late February to early March, 1985) averaging $313 / \mathrm{m}^{3} \pm 229 / \mathrm{m}^{3}$ at Frechette Point, $376 / \mathrm{m}^{3} \pm 207 / \mathrm{m}^{3}$ at Lake $\mathrm{Nicolet,} \mathrm{and} 187 / \mathrm{m}^{3} \pm 152 /$ $\mathrm{m}^{3}$ at Point aux Frenes (Table 31, Appendix 267). Differences in zooplankton abundances among the three locations were statistically significant ( $p=0.05$; Kruskal-Wallis test). Mean current velocity decreased along the course of the river averaging $25.8 \mathrm{~cm} / \mathrm{s}$ at Frechette Point, $18.9 \mathrm{~cm} / \mathrm{s}$ at Lake $\mathrm{Nicolet}$, and $4.0 \mathrm{~cm} / \mathrm{s}$ at Point aux Frenes; differences also were statistically significant ( $p=0.05$; Kruskal-Wallis test).

The zooplankton community was dominated by adult Diaptomus sicilis (mean individual dry weight $10.4 \mu \mathrm{g}$ ) and by adult Limnocalanus macrurus (mean individual dry weight of $37.6 \mu \mathrm{g}$ ). Nauplii, immature copepodites, and cladocerans were extremely rare. There were no differences in the average individual dry weights of zooplankton among the three locations during winter; average individual dry weights ranged from $10.8 \mu \mathrm{g}$ at Lake $\mathrm{Nicolet}$ to $11.0 \mathrm{\mu g}$ at Frechette Point and Point aux Frenes.

Zooplankton distributions were not uniform in the water column. Abundances (based on No. 2-mesh net collections) were significantly different ( $p=0.05$; Mann-Whitney U-test) between channel stations and shallower stations (Table 32 ). In all instances, zooplankton densities were greater in the more rapidly flowing and deeper channel stations than in more shallow waters. There was no apparent difference in mean zooplankton size between shallow and channel stations ( $11.2 \mu \mathrm{g}$ versus $10.8 \mu \mathrm{g}$ at Frechette Point; $10.8 \mu \mathrm{g}$ versus $10.8 \mu \mathrm{g}$ at Lake Nicolet; 10.6 versus 11.0 $\mu g$ at Point aux Frenes). This suggests that while zooplankton abundances varied between channel and shallow stations, community structure did not. Current velocities (Table 32 ) were significantly ( $p=0.05$; Mann-Whitney U-test) higher at channel stations than at shallow stations. 


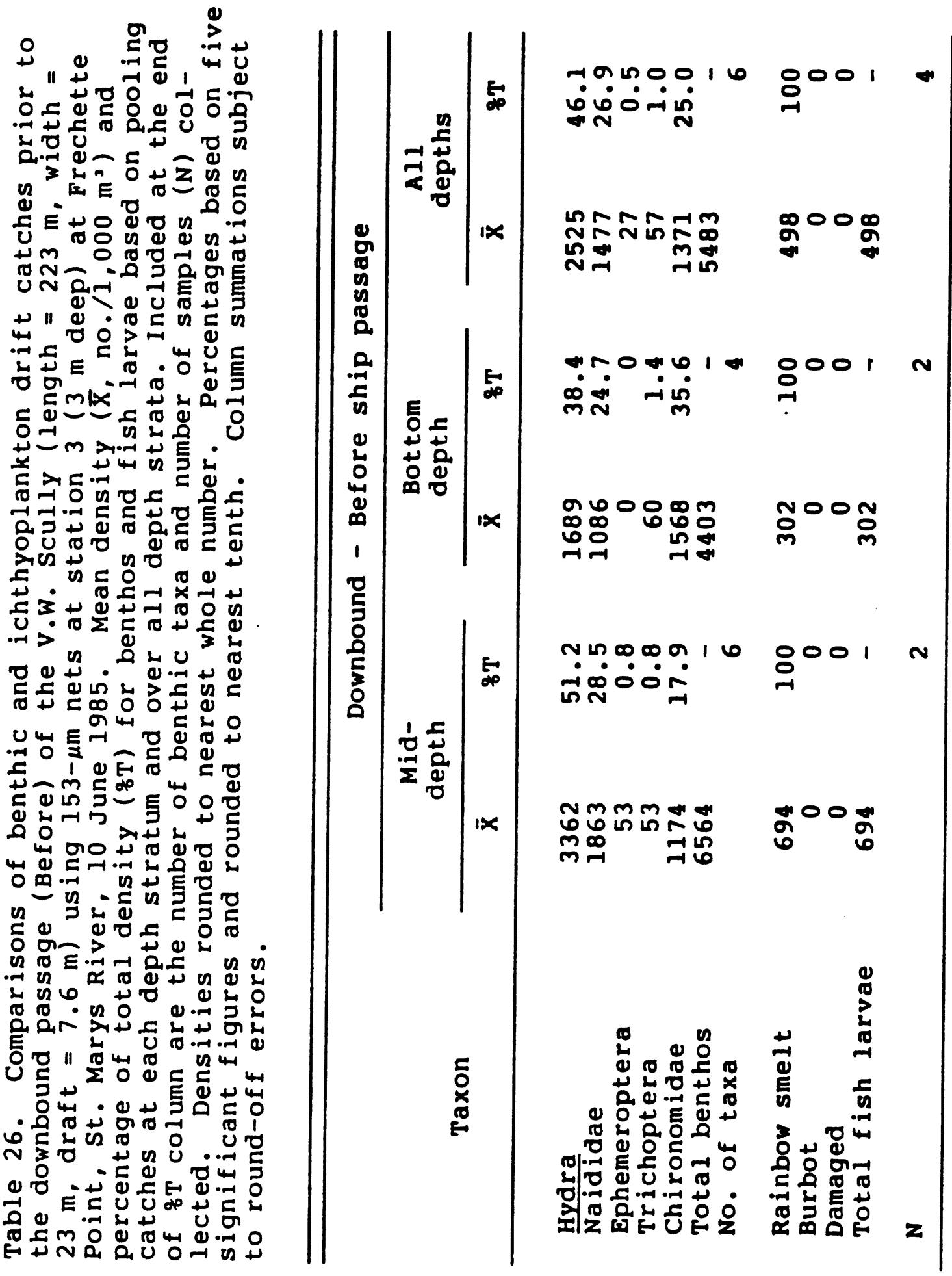




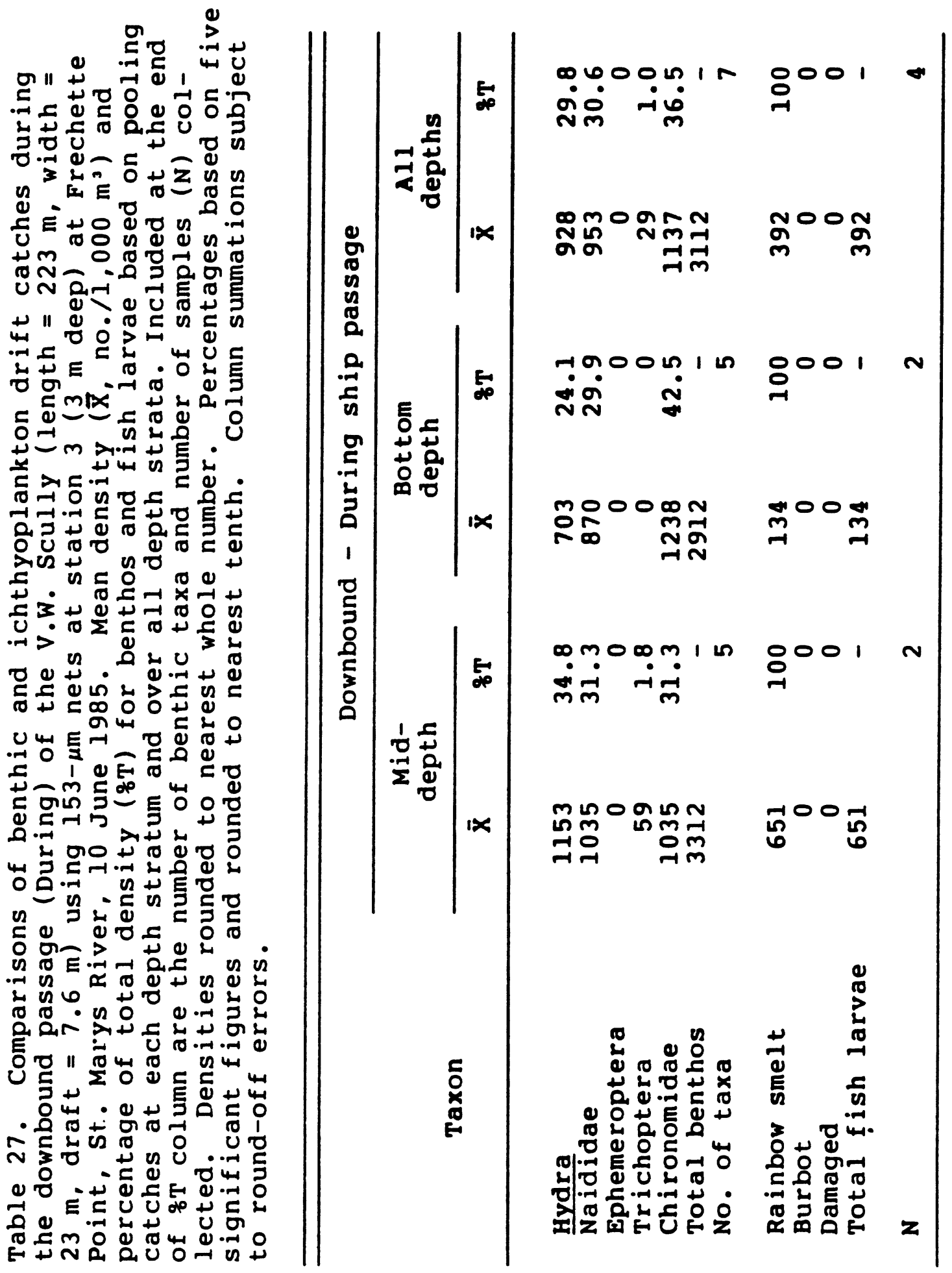



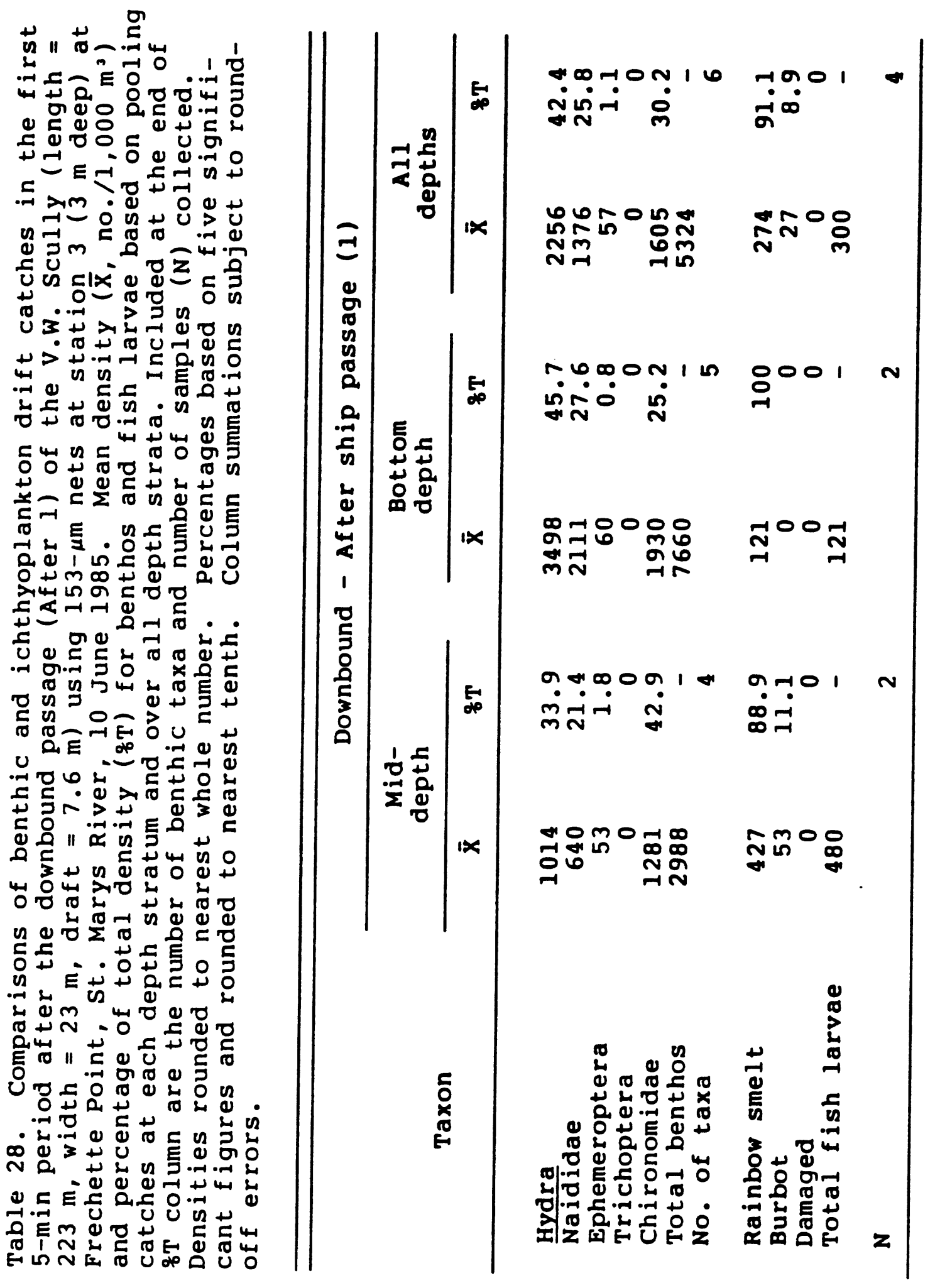

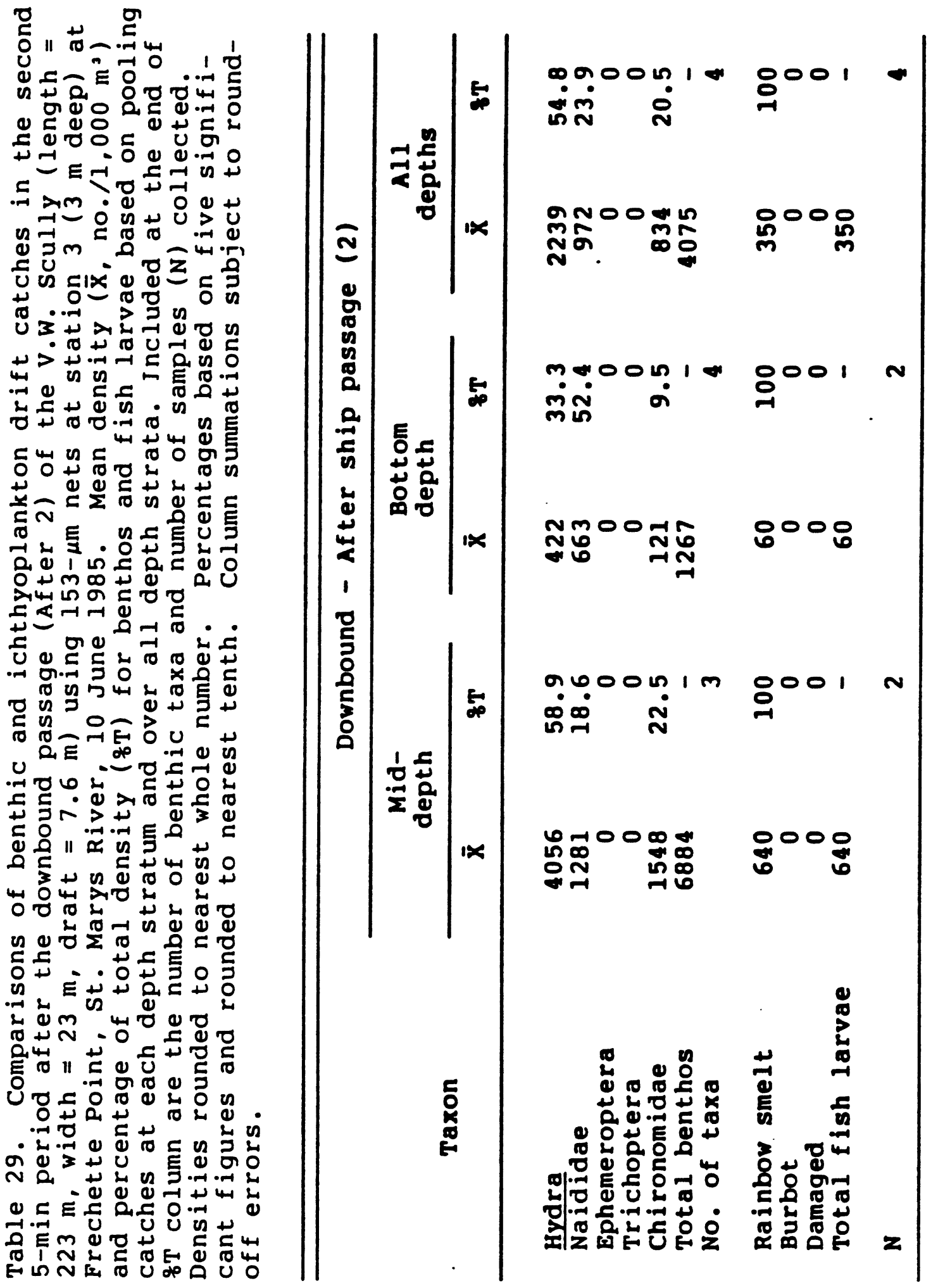


Table 31. Mean zooplankton abundance (No. $/ \mathrm{m}^{3}$ ) and standard deviation in parentheses at Frechette Point, Lake Nicolet, and Point aux Frenes, 26 February3 March 1985 and 2-13 June 1985. All estimates based on No. 2-mesh net collections.

Frechette Point

Feb-Mar

Jun
$313.8 \quad(228.9)$

$172.2(119.6)$
Lake Nicolet

$\begin{array}{rr}376.3 & (206.6) \\ 89.4 & (113.9)\end{array}$
Pointe aux Frenes

$187.3(151.5)$

Table 32. Mean zooplankton abundance $\left(\right.$ No. $\left./ \mathrm{m}^{3}\right)$, current velocity $(\mathrm{cm} / \mathrm{s})$, and weather conditions at channel and shallow stations at Frechette Point, Lake Nicolet, and Point aux Frenes, 26 February-3 March 1985 and 2-13 June 1985. All estimates based on No. 2-mesh net collections. Weather conditions: $1=$ calm, 2 = windy, 3 = very windy.

\begin{tabular}{|c|c|c|c|c|c|c|}
\hline \multirow{2}{*}{ Parameter } & \multicolumn{2}{|c|}{ Frechette Point } & \multicolumn{2}{|c|}{ Lake Nicolet } & \multicolumn{2}{|c|}{ Point aux Frenes } \\
\hline & shallow & Channel & Shallow & Channel & Shallow & Channel \\
\hline \multicolumn{7}{|l|}{ Feb-Mar } \\
\hline$\overline{\text { Zooplank ton }}$ & 264.0 & 476.8 & 321.1 & 468.3 & 152.5 & 245.2 \\
\hline Current & 20.5 & 43.5 & 13.8 & 27.3 & 2.8 & 6.0 \\
\hline Wea ther & - & - & - & - & - & - \\
\hline \multicolumn{7}{|l|}{ Jun } \\
\hline Zooplankton & 149.5 & 247.7 & 87.3 & 93.7 & 25.4 & 6.7 \\
\hline Current & 29.6 & 49.3 & 11.0 & 19.4 & 14.8 & 24.9 \\
\hline Wea ther & 1.5 & 1.0 & 1.5 & 1.1 & 2.1 & 2.0 \\
\hline
\end{tabular}

Zooplankton abundances in No. 2-mesh net collections varied significantly among depth strata (i.e., surface, mid-depth, and bottom). at the three locations. Overall, there was a strong tendency for zooplankton abundances to decrease with decreasing depth; current velocity also decreased with depth (Table 33). At Frechette Point, mean abundances decreased from $750 / \mathrm{m}^{3}$ in surface samples, to $316 / \mathrm{m}^{3}$ in mid-depth samples, to $248 / \mathrm{m}^{3}$ in bottom samples. These differences were statistically significant ( $p=$ 0.05 ; Kruskal-Wallis test) as were differences in zooplankton abundances between mid-depth and bottom samples ( $p=0.05$; MannWhitney U-test). The decrease in current velocity with depth also was statistically significant for both three-depth (surface, mid-depth, and bottom) and two-depth (mid-depth and bottom) strata comparisons. At Lake Nicolet, decreases in zooplankton abundance and current velocity with depth strata were statistically significant for three-depth and two-depth comparisons. At Point aux Frenes, zooplankton abundances varied significantly with depth strata both for two-depth and threedepth comparisons. Differences in current velocity with depth strata were significant only for the three-depth comparisons. 
Table 33. Mean zooplankton abundance (No. $/ \mathrm{m}^{3}$ ) by depth strata and average current velocity $(\mathrm{cm} / \mathrm{s})$ at Frechette Point, Lake Nicolet, and Point aux Frenes, 26 February-3 March 1985 and 2-13 June 1985. All estimates based on No. 2-mesh net collections.

\section{Parameter}

Feb-Mar

Frechette Pt.

Lk. Nicolet

Pt. aux Frenes

Jun

Frechette Pt.

Lk. Nicolet

$P t$. aux Frenes

\section{$\frac{\text { Surface }}{\text { Zoo- Cur- }}$ \\ plankton rent}

$$
\begin{array}{rr}
750.3 & 40.0 \\
611.3 & 24.6 \\
204.8 & 5.7
\end{array}
$$

$\begin{array}{rr}238.6 & 52.2 \\ 116.0 & 20.7 \\ 5.9 & 26.2\end{array}$

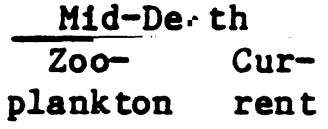

$\begin{array}{rr}316.6 & 28.9 \\ 423.8 & 20.7 \\ 253.9 & 4.3\end{array}$

197.7

129.4

14.2
36.4

14.8

17.3

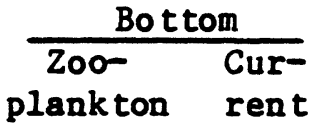

248.2

281.9

21.5

133.0

16.1

3.3

144.4

29.9

$52.4 \quad 11.6$

$28.2 \quad 15.2$

There were no apparent differences in the mean individual sizes of zooplankton collected from the three depth strata at Frechette Point $(10.7 \mu \mathrm{g}, 11.2 \mu \mathrm{g}$, and $11.1 \mu \mathrm{g})$, Lake $\mathrm{Nicolet}(10.8 \mu \mathrm{g}$, $10.9 \mu \mathrm{g}$, and $10.7 \mu \mathrm{g})$, and Point aux Frenes $(10.9 \mu \mathrm{g}, 11.0 \mathrm{\mu g}$, and $10.9 \mathrm{\mu g})$. This suggests that there were only minor changes in zooplankton community structure with depth strata.

Zooplankton did not exhibit significant $(p=0.05$; Mann Whitney U-test) variations in abundance between day and $n i g h t$ No. 2-mesh net collections (Table 34) at any of the three locations. Mean animal size also was similar during day and night collections at Frechette Point ( $10.9 \mu \mathrm{g}$ and $11.2 \mathrm{\mu g})$, Lake Nicolet $(10.9 \mathrm{\mu g}$ and $10.8 \mathrm{\mu g})$, and Point aux Frenes ( $11.0 \mathrm{\mu g}$ and $11.0 \mathrm{\mu g})$ suggesting that zooplankton community structure was similar in day and night collections. Differences in current velocities (Table 34 ) between day and night collections were not statistically significant.

Finally, zooplankton abundances were compared in matched No. 10-mesh and No. 2-mesh net collections. Zooplankton abundances were significantly $(0=0.05$; Mann-Whitney U-test) higher in No. 10-mesh than in No. 2-mesh net collections at Frechette Point $\left(9.56 / \mathrm{m}^{3}\right.$ versus $\left.326 / \mathrm{m}^{3}\right)$ and Lake Nicolet $\left(769 / \mathrm{m}^{3}\right.$ versus $\left.404 / \mathrm{m}^{3}\right)$. However, such differences were not statistically significant at Point aux Frenes $\left(183 / \mathrm{m}^{3}\right.$ versus $\left.210 / \mathrm{m}^{3}\right)$. The mean size of zooplankton collected by the No. 10-mesh net tended to be smaller than for No. 2-mesh net collections, averaging respectively $9.3 \mathrm{\mu g}$ and $10.7 \mathrm{\mu g}$ at Frechette Point, $9.3 \mathrm{\mu g}$ and $10.8 \mathrm{\mu g}$ at Lake Nicolet, and $8.0 \mathrm{\mu g}$ and $11.2 \mathrm{\mu g}$ at Point aux Frenes. 
Table 34. Mean zooplankton abundance $\left(\right.$ No. $\left./ \mathrm{m}^{3}\right)$, current velocity $(\mathrm{cm} / \mathrm{s})$, and weather conditions during day and night collections at Frechette Point, Lake Nicolet, and Point aux Frenes, 26 February-3 March 1985 and 2-13 June 1985. All estimates based on No. 2-mesh net collections. Weather conditions: $1=$ calm, 2 = windy, $3=$ very windy.

\begin{tabular}{|c|c|c|c|c|c|c|}
\hline \multirow[b]{2}{*}{ Parame ter } & \multirow{2}{*}{\multicolumn{2}{|c|}{$\frac{\text { Frechette Point }}{\text { Day Night }}$}} & \multicolumn{2}{|c|}{ Lake Nicolet } & \multicolumn{2}{|c|}{ Point aux Frenes } \\
\hline & & & Day & Night & Day & Night \\
\hline $\begin{array}{l}\text { Feb-Mar } \\
\text { Zooplankton } \\
\text { Current } \\
\text { Wea ther }\end{array}$ & $\begin{array}{c}329.4 \\
26.4 \\
-\end{array}$ & $\begin{array}{c}296.8 \\
25.2 \\
-\end{array}$ & $\begin{array}{r}421.1 \\
18.7 \\
-\end{array}$ & $\begin{array}{c}331.6 \\
19.1 \\
-\end{array}$ & $\begin{array}{r}160.6 \\
4.0 \\
-\end{array}$ & $\begin{array}{r}213.9 \\
4.0 \\
-\end{array}$ \\
\hline $\begin{array}{l}\text { Jun } \\
\text { Zooplank ton } \\
\text { Current } \\
\text { Wea ther }\end{array}$ & $\begin{array}{r}228.4 \\
38.4 \\
2.0\end{array}$ & $\begin{array}{r}118.1 \\
30.1 \\
1.0\end{array}$ & $\begin{array}{r}107.6 \\
15.5 \\
1.3\end{array}$ & $\begin{array}{r}71.7 \\
12.2 \\
1.0\end{array}$ & $\begin{array}{r}3.1 \\
18.9 \\
2.2\end{array}$ & $\begin{array}{r}39.1 \\
15.4 \\
2.0\end{array}$ \\
\hline
\end{tabular}

Spring--

The second sampling was in early June. Zooplankton abundances as determined by No. 2-mesh net collections (Table 3I) were significantly lower in June ( $p=0.05$; Mann-Whitney U-test) at each of the three stations than in February. Abundances in June averaged $172 / \mathrm{m}^{3}$ at Frechette Point, $89.4 / \mathrm{m}^{3}$ at Lake $\mathrm{Nicolet}$, and $21.1 / \mathrm{m}^{3}$ at Lake Munuscong. Differences in zooplankton abundance among the three locations also were statistically significant ( $p=0.05$; Kruskal-Wallis test). Average flow velocity decreased from Frechette Point $(34.2 \mathrm{~cm} / \mathrm{s})$ to Lake Nicolet $(13.9 \mathrm{~cm} / \mathrm{s})$ and then increased at Point aux Frenes (17.1 $\mathrm{cm} / \mathrm{s})$. Differences in flow velocity also were statistically significant ( $p=0.05$; Kruskal-Wallis test) among the three stations.

The zooplankton community was more diverse in early June than in February. At Frechette Point and Lake Nicolet, adult Diaptomus sicilis (mean individual dry weight of $12.6 \mathrm{\mu g}$ ) continued to be a dominant species. Immature Limnocalanus macrurus copepodites (mean individual dry weight of $11.4 \mu \mathrm{g}$ ) also were abundant at these two stations. Other abundant taxa included nauplii, immature cyclops (mean individual dry weight of $1.7 \mu \mathrm{g})$ and Diaptomus spp. $(0.9 \mathrm{\mu g})$ copepodites. Immature Cyclops spp. copepodites generally were the numerically dominant taxon at Point aux Frenes. Mesocyclops sp. and cladocerans such as Daphnia, Bosmina longirostris, and Eubosmina coregoni also were abundant at some stations. Small numbers of the Daphnia morph, D. minnehaha, were present at station 3 at Frechette Point. It probably entered the site from a local stream or small river rather than from lake Superior; the morph has not been recorded from the Great Lakes. Small numbers of epibenthic species were present in the various collections suggesting that 
the littoral zooplankton community was well developed. Mean individual zooplankton dry weight averaged $11.9 \mathrm{\mu g}$ at Frechette Point, $11.7 \mu \mathrm{g}$ at Lake Nicolet, but only $1.9 \mu \mathrm{g}$ at Point aux Frenes. Thus, zooplankton were not only less abundant at point aux Frenes than at the two upriver stations, but were smaller. This suggests that the downriver decrease in zooplankton abundance was most strongly associated with the larger-bodied taxa.

As in winter, differences in zooplankton abundance (based on No. 2-mesh net collections) between channel and shallow stations were significant $(p=0.05$; Mann-Whitney U-test). At Frechette Point and Lake Nicolet, zooplankton were more abundant at channel stations than at stations located in more nearshore, shallow regions (Table 32). There was no apparent difference in mean animal size between channel and shallow stations both at Frechette Point $(12.1 \mu \mathrm{g}$ and $11.9 \mathrm{\mu g})$ and Lake Nicolet $(11.7 \mathrm{\mu g}$ and $11.7 \mu \mathrm{g})$; this suggests that zooplankton community structure was similar in channel and shallow areas. Current velocities were significantly higher at channel stations than shallow stations (Table 32). At Point aux Frenes, abundances were significantly greater in shallow $\left(25 / \mathrm{m}^{3}\right)$ than channel $\left(7 / \mathrm{m}^{3}\right)$ stations. Furthermore, zooplankton tended to be smaller at shallow stations $(1.7 \mu \mathrm{g})$ than in the channel $(4.0 \mu \mathrm{g})$. This suggests that there were large differences in zooplankton community structure between shallow and channel stations. As at the other two upriver locations, current velocity was significantly higher in the channel than in the shallow regions of the river.

Differences in zooplankton abundances (No. 2-mesh net collections) with sample depth were significant ( $p=0.05$; KruskalWallis test for surface, mid-depth, and bottom comparisons; MannWhitney U-test for mid-depth versus bottom comparisons). At Frechette Point, mean abundances decreased with sample depth as did current velocities (Table 33); differences were statistically significant for all comparisons. There was littie change in mean zooplankton size with increasing depth strata $(12.3 \mu \mathrm{g}, 11.9 \mu \mathrm{g}$, and $11.9 \mu \mathrm{g}$, respectively). Differences in zooplankton abundance and current velocity with depth strata were statistically significant for all comparisons at Lake Nicolet; lowest zooplankton abundances and current vel-ocities were observed near the river bottom. Mean zooplankton size showed little change with increasing depth strata (from $11.7 \mu g$ to $11.9 \mu g$ to 11.1 $\mu g)$. Differences in zooplankton abundance with depth strata were not statistically significant at Point aux Frenes; differences in current velocity were significant among the three depth strata but not for mid-depth versus bottom com-parisons. There was a strong trend for mean zooplankton size to decrease with depth strata, i.e., from $4.7 \mu \mathrm{g}$ to $2.2 \mu \mathrm{g}$ to $1.7 \mu \mathrm{g}$. 
In contrast with winter zooplankton, summer zooplankton exhibited significant ( $p=0.05$; Mann-Whitney U-test) differences in abundance with sampling time based on No. 2-mesh net collections. At Frechette Point, zooplankton were more abundant in day $\left(228 / \mathrm{m}^{3}\right)$ than night $\left(118 / \mathrm{m}^{3}\right)$ collections (Table 34$)$. Greatest abundances during daylight hours were not anticipated. Mean animal size was $12.0 \mu \mathrm{g}$ during day collections and $11.9 \mu \mathrm{g}$ during night collections suggesting that there were little diel changes in zooplankton community structure. Diel differences in zooplankton abundance may have been related to the higher current flow $(38.4 \mathrm{~cm} / \mathrm{s}$ versus $30.1 \mathrm{~cm} / \mathrm{s})$ and generally more windy collection periods ( 2.0 versus 1.0 , where $1=\operatorname{calm}, 2=$ windy, and 3 = very windy) associated with day versus night collections (Table 34); these differences were statistically significant. Similarly, zooplankton were significantly more abundant in day $\left(421 / \mathrm{m}^{3}\right)$ than night $\left(332 / \mathrm{m}^{3}\right)$ collections made at Lake $\mathrm{Nicolet}$ (Table 34); mean animal size averaged $11.7 \mu \mathrm{g}$ during day and night collections. Again, average current flow was significantly higher during day $(15.5 \mathrm{~cm} / \mathrm{s})$ than night collections $(12.2 \mathrm{~cm} / \mathrm{s})$; the weather was also significantly more windy during day collections (weather conditions of 1.3 and 1.0 respectively for day and night sampling). Only at point aux Frenes, where the zooplankton community was dominated by small taxa, were zooplankton significantly more abundant in $\mathrm{night}\left(39.1 / \mathrm{m}^{3}\right)$ than day $\left(3.1 / \mathrm{m}^{3}\right)$ collections (Table 34 ): Animals tended to be smaller in night collections averaging $1.7 \mathrm{\mu g}$ versus $4.0 \mathrm{\mu g}$ during the day. Although the average current flow was significantly higher during day $(18.9 \mathrm{~cm} / \mathrm{s})$ than night $(15.4 \mathrm{~cm} /$ s) collections (Table 34), there was no significant difference in weather conditions. The weather was windy to very windy during the day (average weather condition of 2.2 ) and was windy (average weather condition of 2.0 ) at night.

Zooplankton densities were significantly (Mann-Whitney Utest) more abundant in No. 10-mesh net collections than in No. 2mesh net collections, averaging respectively $542 / \mathrm{m}^{3}$ and $241 / \mathrm{m}^{3}$ at Frechette Point, $284 / \mathrm{m}^{3}$ and $73 / \mathrm{m}^{3}$ at Lake Nicolet, and $439 / \mathrm{m}^{3}$ and $9 / \mathrm{m}^{3}$ at Point aux Frenes (Table 35). At Frechette Point, where there was only a 2.3-fold difference in zooplankton abundance between the two collection methods, the mean size of animals was large averaging $12.1 \mathrm{\mu g}$ for the No. 2-mesh net and $7.0 \mathrm{\mu g}$ for the No. 10-mesh net. However, at Point aux Frenes, where there was a 50-fold difference in abundance estimates between the two nets, zooplankton were considerably smaller, averaging only $2.6 \mathrm{\mu g}$ in the No. 2-mesh-net collections and $1.3 \mu \mathrm{g}$ in the No. 10-mesh net. 
Table 35. Mean zooplankton density (No./m $/ \mathrm{m}^{3}$ ) and mean animal dry weight ( $\mu \mathrm{g}$ ) in No. 2-mesh and No. 10-mesh net collectlons made at Frechette Point, Lake Nicolet, and Point aux Frenes, 26 February-3 March 1985 and 2-13 June 1985.

\begin{tabular}{|c|c|c|c|c|c|c|}
\hline \multirow[b]{2}{*}{ Net Size } & \multicolumn{2}{|c|}{ Frechette Point } & \multicolumn{2}{|c|}{ Lake Nicolet } & \multirow{2}{*}{$\frac{\text { Point aux }}{\text { Dens1ty }}$} & \multirow{2}{*}{$\frac{\text { Frenes }}{\text { Welght }}$} \\
\hline & Dens Ity & Welght & Dens1t & Weight & & \\
\hline $\begin{array}{l}\text { Feb-Mar } \\
\text { No. } 2 \\
\text { No. } 10\end{array}$ & $\begin{array}{l}362.6 \\
956.2\end{array}$ & $\begin{array}{r}10.7 \\
9.3\end{array}$ & $\begin{array}{l}403.9 \\
769.1\end{array}$ & $\begin{array}{r}10.8 \\
9.3\end{array}$ & $\begin{array}{l}210.3 \\
183.0\end{array}$ & $\begin{array}{r}11.2 \\
8.0\end{array}$ \\
\hline $\begin{array}{l}\frac{\text { Jun }}{\text { No. } 2} \\
\text { No. } 10\end{array}$ & $\begin{array}{l}240.7 \\
541.6\end{array}$ & $\begin{array}{r}12.1 \\
7.0\end{array}$ & $\begin{array}{r}72.8 \\
283.7\end{array}$ & $\begin{array}{r}11.7 \\
3.7\end{array}$ & $\begin{array}{r}8.8 \\
438.7\end{array}$ & $\begin{array}{l}2.6 \\
1.3\end{array}$ \\
\hline
\end{tabular}

Biomass Studies

Overview--

The general purpose of the biomass investigations was to characterize the drift or load of particulates carried by the St. Marys River. Load was estimated in terms of dry weight and ash-free weight. Dry weight and ash-free weight were estimated by direct weighing of the appropriate samples (seston, plants, benthos, mysids) or from estimates based on taxa abundance and separately determined dry weight of selected individuals (zooplankton and $f i s h$ ). An average ash-free weight conversion factor of 0.047 (determined in the laboratory) was used for $f$ ish and a factor of 0.045 (the mean value determined for mysids in the laboratory) was used for zooplankton. Detritus dry weight was estimated by subtracting estimated zooplankton dry weight from seston dry weight. Although this estimate generally was good, some negative values were calculated. This sampling error occurred when the seston was strongly dominated by zooplankton. Since different subsamples were used for zooplankton abundance estimates and seston determinations, small variations in subsample weights resulted in some negative detritus dry-weight estimates. These sampling errors were further magnified when detritus ash-free weight was estimated.

In the following paragraphs, biomass data are presented as dry weight for zooplankton, plants, benthos (including mysids), seston, and detritus. The percentage of dry weight that was ashfree weight is also given for seston (\&AFW:seston). Ash-free weights are not presented for other components of the river load because a constant conversion factor was used for zooplankton (a dominant component of river load) and $f i s h$, and because the detritus ash-free estimates were not sufficiently accurate to warrant discussion. 
General Composition of River Load--

The average load carried by the St. Marys River was 5,406 $\mathrm{mg} / 1,000 \mathrm{~m}^{3}$ in February and $11,129 \mathrm{mg} / 1,000 \mathrm{~m}^{3}$ in early June (Table 36, Appendixes 135-261). Seston was the major component of the load during both sampling seasons with zooplankton (63.2\%) predominating in February and detritus $(86.9 \%)$ in June. As expected, fAFW:seston was lower in winter $(4.8 \%)$ than in spring $(25.6 \%)$. Plants were minor contributors to total river load with the absolute and relative contribution increasing in spring. Benthos also was a minor contributor to river load both in winter and spring. Mysids were more abundant in winter than in spring, contributing to a greater percentage of winter (31.7\%) than spring $(5.1 \%)$ benthic biomass. Fish larvae were not present in winter collections and were minor components $(0.04 \%)$ of spring collections. Differences in all components of river load were statistically significant $(p=0.05$; Mann-Whitney U-test) between winter and spring.

\section{River Load During Winter--}

There were statistically significant ( $p=0.05$; KruskalWallis test) differences in dry weight for all components (except mysids) of river load among the three stations; comparisons were based on No. 2-mesh net collections. Average total river load was $6,835 \mathrm{mg} / 1,000 \mathrm{~m}^{3}$ at Frechette Point, 5,619 $\mathrm{mg} / 1,000 \mathrm{~m}^{3}$ at Lake Nicolet, and $2,687 \mathrm{mg} / 1,000 \mathrm{~m}^{3}$ at Point aux Frenes (Table 37; Appendixes 135-154, 175-200, 229-241). The major component

Table 36. Mean concentration (mg dry weight/1,000 $\mathrm{m}^{3}$ ) and percent composition of the various components of river drift, seston percent (of dry weight), ashfree weight (AFW), and current velocity $(\mathrm{cm} / \mathrm{s})$ during 26 February-3 March 1985 and 2-13 June 1985. All estimates based on No. 2-mesh net collections. Mysis weights-are included in the total benthos weight. Detritus weights are the difference between seston and zooplankton weights.

\begin{tabular}{lcccc}
\hline \hline \multirow{2}{*}{ Parame ter } & \multicolumn{2}{c}{ Winter } & \multicolumn{2}{c}{ Spring } \\
\cline { 2 - 5 } \cline { 2 - 5 } & Concentration & Percent & Concentration & Percent \\
\hline Zooplankton & $3,416.3$ & 63.2 & $1,047.8$ & 9.4 \\
Plants & 22.6 & 0.4 & 313.1 & 2.8 \\
Total benthos & 45.1 & 0.8 & 92.5 & 0.8 \\
Mysis & 14.3 & 0.3 & 4.7 & 0.04 \\
Fish & 0.0 & 0.0 & 4.8 & 0.04 \\
Seston & $5,338.9$ & 98.8 & $10,719.3$ & 96.3 \\
Detr1tus & $1,922.6$ & 35.6 & $9,671.5$ & 86.9 \\
Total & $5,406.6$ & & $11,129.7$ & \\
\% AFW: ses ton & 4.8 & & 25.6 & \\
Current & 18.1 & & 20.8 & \\
\hline
\end{tabular}


Table 37. Mean concentration ( $\mathrm{mg}$ dry weight/1,000 $\mathrm{m}^{3}$ ) and percent composition of the various components of river drift, seston percent (of dry weight), ashfree weight (AFW), current velocity $(\mathrm{cm} / \mathrm{s})$, and weather conditions by location during 26 February-3 March 1985 and 2-13 June 1985. All estimates based on No. 2-mesh net collections. Weather conditions: $1=$ calm, $2=$ windy, $3=$ very windy. Mysis weights are included in the total benthos weight. Detritus weights are the difference between seston and zooplankton weights.

\begin{tabular}{|c|c|c|c|c|c|c|}
\hline \multirow[b]{2}{*}{ Parameter } & \multicolumn{2}{|c|}{ Frechette Point } & \multicolumn{2}{|l|}{ Lake Nicolet } & \multicolumn{2}{|c|}{ Point aux Frenes } \\
\hline & Concentration & $\%$ & Concentration & $\%$ & Concentration & $\%$ \\
\hline $\begin{array}{l}\text { Feb-Mar } \\
\text { Zooplankton } \\
\text { Plants } \\
\text { Benthos } \\
\text { Mysis } \\
\text { Fish } \\
\text { Seston } \\
\text { Detritus } \\
\text { Total }\end{array}$ & $\begin{array}{r}3,449.4 \\
73.0 \\
87.4 \\
8.2 \\
0.0 \\
6,674.8 \\
3,225.4 \\
6,835.2\end{array}$ & $\begin{array}{r}50.5 \\
1.1 \\
1.3 \\
0.1 \\
0.0 \\
97.7 \\
47.2\end{array}$ & $\begin{array}{r}4,069.6 \\
3.0 \\
30.6 \\
25.4 \\
0.0 \\
5,585.1 \\
1,515.5 \\
5,618.7\end{array}$ & $\begin{array}{r}72.4 \\
0.1 \\
0.5 \\
0.5 \\
0.0 \\
99.4 \\
27.0\end{array}$ & $\begin{array}{r}2,056.2 \\
5.1 \\
6.3 \\
2.0 \\
0.0 \\
2,675.8 \\
619.6 \\
2,687.2\end{array}$ & $\begin{array}{r}76.5 \\
0.2 \\
0.2 \\
0.1 \\
0.0 \\
99.6 \\
23.1\end{array}$ \\
\hline $\begin{array}{l}\text { \% AFW: seston } \\
\text { Current }\end{array}$ & $\begin{array}{r}6.4 \\
25.8\end{array}$ & & $\begin{array}{r}3.8 \\
18.9\end{array}$ & & $\begin{array}{l}4.5 \\
4.0\end{array}$ & \\
\hline \multicolumn{7}{|l|}{ Jun } \\
\hline $\begin{array}{l}\text { Zooplankton } \\
\text { Plants } \\
\text { Benthos } \\
\text { Mysis } \\
\text { Fish } \\
\text { Seston } \\
\text { Detritus } \\
\text { Total }\end{array}$ & $\begin{array}{r}2,056.6 \\
984.3 \\
67.2 \\
6.6 \\
1.7 \\
22,094.0 \\
20,037.4 \\
23,147.2\end{array}$ & $\begin{array}{r}8.9 \\
4.3 \\
0.3 \\
0.0 \\
0.0 \\
95.5 \\
86.6\end{array}$ & $\begin{array}{r}1,047.1 \\
22.2 \\
122.4 \\
6.6 \\
10.4 \\
1,868.7 \\
821.6 \\
2,023.7\end{array}$ & $\begin{array}{r}51.7 \\
1.1 \\
6.1 \\
0.3 \\
0.5 \\
92.3 \\
40.6\end{array}$ & $\begin{array}{r}40.2 \\
33.8 \\
76.8 \\
0.0 \\
0.1 \\
11,618.2 \\
11,578.0 \\
11,728.9\end{array}$ & $\begin{array}{r}0.3 \\
0.3 \\
0.7 \\
0.0 \\
0.0 \\
99.1 \\
98.7\end{array}$ \\
\hline $\begin{array}{l}\text { \% AFW : seston } \\
\text { Current } \\
\text { Wea ther }\end{array}$ & $\begin{array}{r}26.7 \\
34.2 \\
1.5\end{array}$ & & $\begin{array}{r}24.6 \\
13.9 \\
1.1\end{array}$ & & $\begin{array}{r}26.9 \\
17.1 \\
2.1\end{array}$ & \\
\hline
\end{tabular}


of river load was zooplankton with the percentage increasing downriver as detritus concentrations decreased. Plant and benthic biomass also decreased downriver. Average current velocity decreased from $25.8 \mathrm{~cm} / \mathrm{s}$ at Frechette Point, to $18.9 \mathrm{~cm} /$ $s$ at Lake Nicolet, to $4.0 \mathrm{~cm} / \mathrm{s}$ at Point aux Frenes. Although average current velocity was considerably lower at point aux Frenes than at Lake Nicolet, river load composition was similar with detritus accounting for approximately $23 \%$ to $27 \%$ of the load.

Total river load was higher in the channels than in the shallows (Table 38); such differences were significant $(p=0.05$; Mann-Whitney U-test) at all three locations. Since zooplankton was the major component of river load (and was more abundant at channel stations, Table 32), the greater river load in channel than shallow stations was expected. Detritus concentrations also were significantly higher in the channels as were current velocities. Differences in plant, benthos, and mysid densities between channel and shallow stations were not statistically significant ( $p=0.05$; Mann-Whitney U-test and median test).

River load concentrations varied with depth strata (Table 39). Total river load varied significantly $(p=0.05)$ with depth (Kruskal-Wallis test for surface versus mid-depth versus bottom comparisons; Mann-Whitney U-test for mid-depth versus bottom comparisons). Total river load decreased with depth, reflecting the decrease in zooplankton density and biomass with depth. Current velocity also decreased with depth, with differences statistically significant at all three stations. Detritus concentrations were significantly different for the three depthstrata comparisons but not for mid-depth versus bottom comparisons. Since surface samples were collected only in the channels, significant differences based on three-way but not twoway comparisons probably reflect channel effects. Differences in benthos and mysid densities with depth strata were not statistically significant. Thus, there was no apparent relationship between current velocity among various regions of the water column and benthic drift from the sediments. Differences in plant biomass with depth strata were not statistically significant ( $p=0.05 ;$ Mann-Whitney U-test and median test). Median test results were used for those statistical comparisons where a large number of samples did not contain measurable plant biomass.

River load differed between day and night sampling (Table 40). Day versus night comparisons $(p=0.05$; Mann-Whitney Utest) were statistically significant only for benthos and mysids with biomass increasing at night at all three stations. Current velocities were not significantly different between day and night sampling. This suggests that the greater biomass of benthos and 
Table 38. Mean concentration ( $\mathrm{mg} / 1,000 \mathrm{~m}^{3}$ ) of the various components of river drift, seston percent (of dry weight), ash-free weight (AFW), current velocity $(\mathrm{cm} / \mathrm{s})$, and weather between channel and shallow stations 26 February-3 March 1985 and 2-13 June 1985. All estimates based on No. 2-mesh net collections. Weather conditions: $1=c a l m, 2=$ windy, $3=$ very windy. Mysis weights are included in the total benthos weight. Detritus weights are the difference between seston and zooplankton weights.

\begin{tabular}{|c|c|c|c|c|c|c|}
\hline \multirow[b]{2}{*}{ Parame ter } & \multicolumn{2}{|c|}{ Frechette Point } & \multicolumn{2}{|c|}{ Lake Nicolet } & \multicolumn{2}{|c|}{ Point aux Frenes } \\
\hline & Shallow & Channel & Shallow & Channel & Shallow & Channel \\
\hline $\begin{array}{l}\text { Feb-Mar } \\
\text { Zooplankton } \\
\text { Plants } \\
\text { Benthos } \\
\text { Mysis } \\
\text { Fish } \\
\text { Seston } \\
\text { Detritus } \\
\text { Total }\end{array}$ & $\begin{array}{r}2,960.4 \\
80.4 \\
87.2 \\
8.0 \\
0.0 \\
5,597.3 \\
2,636.9 \\
5,764.9\end{array}$ & $\begin{array}{r}5,079.2 \\
58.3 \\
87.9 \\
8.8 \\
0.0 \\
10,266.7 \\
5,187.5 \\
10,412.9\end{array}$ & $\begin{array}{r}3,428.6 \\
0.1 \\
40.5 \\
33.6 \\
0.0 \\
4,841.8 \\
1,359.2 \\
4,916.0\end{array}$ & $\begin{array}{r}5,047.9 \\
7.7 \\
14.2 \\
11.7 \\
0.0 \\
6,823.9 \\
1,760.9 \\
6,845.8\end{array}$ & $\begin{array}{r}1,670.8 \\
8.0 \\
6.7 \\
0.6 \\
0.0 \\
1,976.7 \\
305.9 \\
1,991.4\end{array}$ & $\begin{array}{r}2,698.5 \\
0.4 \\
5.6 \\
4.4 \\
0.0 \\
3,841.0 \\
1,142.5 \\
3,847.0\end{array}$ \\
\hline $\begin{array}{l}\text { \% AFW: seston } \\
\text { Current }\end{array}$ & $\begin{array}{r}6.4 \\
20.5\end{array}$ & $\begin{array}{r}6.2 \\
43.5\end{array}$ & $\begin{array}{r}3.7 \\
13.8\end{array}$ & $\begin{array}{r}3.9 \\
27.3\end{array}$ & $\begin{array}{l}5.6 \\
2.8\end{array}$ & $\begin{array}{l}2.7 \\
6.0\end{array}$ \\
\hline Jun & & & & & & \\
\hline $\begin{array}{l}\text { Zooplank ton } \\
\text { Plants } \\
\text { Benthos } \\
\text { Mys 1s } \\
\text { P1sh } \\
\text { Seston } \\
\text { Detr1 tus } \\
\text { Total }\end{array}$ & $\begin{array}{r}1,776.1 \\
1,254.7 \\
79.1 \\
6.9 \\
0.9 \\
24,914.1 \\
23,138.4 \\
26,225.7\end{array}$ & $\begin{array}{r}2,991.3 \\
82.9 \\
27.7 \\
5.9 \\
4.3 \\
12,691.8 \\
9,700.5 \\
12,806.7\end{array}$ & $\begin{array}{r}1,022.7 \\
23.8 \\
127.4 \\
3.1 \\
12.4 \\
2,011.1 \\
988.4 \\
2,174.7\end{array}$ & $\begin{array}{r}1,095.8 \\
18.9 \\
112.5 \\
13.7 \\
6.5 \\
1,584.0 \\
488.2 \\
1,721.9\end{array}$ & $\begin{array}{r}44.3 \\
39.5 \\
93.3 \\
0.0 \\
0.1 \\
15,080.5 \\
15,041.0 \\
15,213.4\end{array}$ & $\begin{array}{r}26.7 \\
14.4 \\
20.1 \\
0.0 \\
0.4 \\
219.6 \\
192.9 \\
254.5\end{array}$ \\
\hline $\begin{array}{l}\text { \% AFW: ses ton } \\
\text { Current } \\
\text { Wea ther }\end{array}$ & $\begin{array}{r}28.1 \\
29.6 \\
1.5\end{array}$ & $\begin{array}{r}22.0 \\
49.3 \\
1.0\end{array}$ & $\begin{array}{r}25.1 \\
11.0 \\
1.5\end{array}$ & $\begin{array}{r}23.6 \\
19.4 \\
1.1\end{array}$ & $\begin{array}{r}26.9 \\
14.8 \\
2.1\end{array}$ & $\begin{array}{r}26.8 \\
24.9 \\
2.0\end{array}$ \\
\hline
\end{tabular}


Table 39. Mean concentration ( $\mathrm{mg}$ dry weight/1,000 $\mathrm{m}^{3}$ ) of the various components of river drift, seston percent (of dry weight), ash-free weight (AFW), and current velocity $(\mathrm{cm} / \mathrm{s})$ by depth 8 trata and location, 26 February-3 March 1985 and 2-13 June 1985. All estimates based on No. 2-mesh net collections. Mysis weights are included in the total benthos weight. Detritus weights are the difference between seston and zooplankton weights.

\begin{tabular}{|c|c|c|c|c|c|c|}
\hline \multirow[b]{2}{*}{ Parameter } & \multicolumn{3}{|c|}{ February-March } & \multicolumn{3}{|c|}{ June } \\
\hline & Surface & Mid-depth & Bottom & Surface & Mid-depth & Bottom \\
\hline $\begin{array}{l}\text { Frechette } \\
\text { Zooplankton } \\
\text { Plants } \\
\text { Benthos } \\
\text { Mysis } \\
\text { Fish } \\
\text { Seston } \\
\text { Detritus } \\
\text { Total }\end{array}$ & $\begin{array}{r}7,741.4 \\
72.8 \\
66.2 \\
18.0 \\
0.0 \\
16,378.0 \\
8,636.6 \\
16,517.0\end{array}$ & $\begin{array}{r}3,558.8 \\
51.3 \\
54.6 \\
8.5 \\
0.0 \\
6,549.7 \\
2,290.9 \\
6,655.6\end{array}$ & $\begin{array}{r}2,758.1 \\
86.1 \\
114.2 \\
6.5 \\
0.0 \\
5,378.0 \\
2,619.9 \\
5,578.3\end{array}$ & $\begin{array}{r}2,941.7 \\
37.9 \\
20.1 \\
10.8 \\
0.6 \\
4,378.5 \\
1,436.8 \\
4,437.1\end{array}$ & $\begin{array}{r}2,357.7 \\
2,298.2 \\
50.4 \\
4.3 \\
1.5 \\
9,551.8 \\
7,194.1 \\
11,901.9\end{array}$ & $\begin{array}{r}1,715.0 \\
181.0 \\
85.6 \\
7.7 \\
2.2 \\
33,583.0 \\
31,868.0 \\
33,868.0\end{array}$ \\
\hline $\begin{array}{l}\text { \% AFW: seston } \\
\text { Current }\end{array}$ & $\begin{array}{r}7.7 \\
40.0\end{array}$ & $\begin{array}{r}4.0 \\
28.9\end{array}$ & $\begin{array}{r}7.8 \\
21.5\end{array}$ & $\begin{array}{l}16.1 \\
52.2\end{array}$ & $\begin{array}{l}25.8 \\
36.4\end{array}$ & $\begin{array}{l}28.9 \\
29.9\end{array}$ \\
\hline $\begin{array}{l}\text { Nicolet } \\
\text { Zooplank ton } \\
\text { Plants } \\
\text { Benthos } \\
\text { Mysis } \\
\text { Fish } \\
\text { Seston } \\
\text { Detritus } \\
\text { Total }\end{array}$ & $\begin{array}{r}6,579.6 \\
13.3 \\
6.7 \\
6.1 \\
0.0 \\
8,908.9 \\
2,329.3 \\
8,928.9\end{array}$ & $\begin{array}{r}4,634.2 \\
0.0 \\
23.8 \\
20.0 \\
0.0 \\
6,134.4 \\
1,500.2 \\
6,158.2\end{array}$ & $\begin{array}{r}3,018.6 \\
2.6 \\
41.7 \\
34.3 \\
0.0 \\
4,342.1 \\
1,323.5 \\
4,386.4\end{array}$ & $\begin{array}{r}1,361.6 \\
5.2 \\
45.4 \\
0.4 \\
8.0 \\
1,685.9 \\
324.3 \\
1,744.4\end{array}$ & $\begin{array}{r}1,534.4 \\
15.3 \\
183.3 \\
8.0 \\
16.2 \\
1,926.0 \\
391.7 \\
2,140.8\end{array}$ & $\begin{array}{r}598.2 \\
31.1 \\
92.1 \\
6.9 \\
6.5 \\
1,864.9 \\
1,266.7 \\
1,994.6\end{array}$ \\
\hline $\begin{array}{l}\text { \% AFW: seston } \\
\text { Current }\end{array}$ & $\begin{array}{r}3.7 \\
24.6\end{array}$ & $\begin{array}{r}3.5 \\
20.7\end{array}$ & $\begin{array}{r}4.0 \\
16.1\end{array}$ & $\begin{array}{l}30.8 \\
20.7\end{array}$ & $\begin{array}{l}20.7 \\
14.8\end{array}$ & $\begin{array}{l}26.2 \\
11.6\end{array}$ \\
\hline Point aux Fre & & & & & & \\
\hline $\begin{array}{l}\text { Zooplankton } \\
\text { Plants } \\
\text { Benthos } \\
\text { Mysis } \\
\text { Fish } \\
\text { Seston } \\
\text { Detritus } \\
\text { Total }\end{array}$ & $\begin{array}{r}2,228.4 \\
0.0 \\
8.4 \\
8.2 \\
0.0 \\
3,228.8 \\
1,000.4 \\
3,245.4\end{array}$ & $\begin{array}{r}2,802.5 \\
0.0 \\
2.3 \\
1.3 \\
0.0 \\
3,487.7 \\
685.2 \\
3,490.0\end{array}$ & $\begin{array}{r}1,453.4 \\
10.3 \\
8.7 \\
1.0 \\
0.0 \\
1,930.9 \\
477.5 \\
1,949.9\end{array}$ & $\begin{array}{r}27.9 \\
24.2 \\
2.2 \\
0.0 \\
0.5 \\
171.1 \\
143.2 \\
198.0\end{array}$ & $\begin{array}{r}30.9 \\
13.1 \\
53.5 \\
0.0 \\
0.1 \\
2,334.7 \\
2,303.8 \\
2,401.4\end{array}$ & $\begin{array}{r}48.6 \\
49.9 \\
104.2 \\
0.0 \\
0.1 \\
20,034.4 \\
19,984.4 \\
20,187.2\end{array}$ \\
\hline $\begin{array}{l}\text { \% AFW: seston } \\
\text { Current }\end{array}$ & $\begin{array}{l}3.7 \\
5.7\end{array}$ & $\begin{array}{l}3.5 \\
4.3\end{array}$ & $\begin{array}{l}5.5 \\
3.3\end{array}$ & $\begin{array}{l}29.0 \\
26.2\end{array}$ & $\begin{array}{l}28.6 \\
17.3\end{array}$ & $\begin{array}{l}25.2 \\
15.7\end{array}$ \\
\hline
\end{tabular}


Table 40. Mean concentration ( $\mathrm{mg} / 1,000 \mathrm{~m}^{3}$ ) of the various components of river drift, seston percent (of dry weight), ash-free welght (AFW), current velocity $(\mathrm{cm} / \mathrm{s})$, and weather during the day and night, 26 February-3 March 1985 and 2-13 June 1985. All estimates based on No. 2-mesh net collections. Weather conditions: $1=$ calm, 2 = windy, 3 = very windy. Mysis welghts are included in the total benthos weight. Detritus weights are the difference between seston and zooplankton weights.

\begin{tabular}{|c|c|c|c|c|c|c|}
\hline \multirow[b]{2}{*}{ Parame ter } & \multicolumn{2}{|c|}{ Frechette Point } & \multicolumn{2}{|c|}{ Lake N1colet } & \multicolumn{2}{|c|}{ Point aux Frenes } \\
\hline & Day & Night & Day & Night & Day & Night \\
\hline $\begin{array}{l}\text { Feb-Mar } \\
\text { Zooplankton } \\
\text { Plants } \\
\text { Benthos } \\
\text { Mysis } \\
\text { Fish } \\
\text { Seston } \\
\text { Detritus } \\
\text { Total }\end{array}$ & $\begin{array}{r}3,579.9 \\
42.6 \\
7.9 \\
0.0 \\
0.0 \\
6,538.8 \\
2,960.9 \\
6,589.3\end{array}$ & $\begin{array}{r}3,320.9 \\
97.6 \\
168.4 \\
16.5 \\
0.0 \\
6,810.9 \\
3,490.0 \\
7,076.9\end{array}$ & $\begin{array}{r}4,568.8 \\
5.5 \\
11.4 \\
8.3 \\
0.0 \\
5,868.5 \\
1,999.8 \\
5,885.4\end{array}$ & $\begin{array}{r}3,570.6 \\
0.4 \\
49.8 \\
42.5 \\
0.0 \\
5,301.6 \\
1,731.0 \\
5,351.9\end{array}$ & $\begin{array}{r}1,761.8 \\
0.0 \\
5.3 \\
0.0 \\
0.0 \\
2,270.9 \\
509.1 \\
2,276.2\end{array}$ & $\begin{array}{r}2,350.6 \\
10.3 \\
7.2 \\
4.2 \\
0.0 \\
3,080.7 \\
730.9 \\
3,098.2\end{array}$ \\
\hline $\begin{array}{l}\text { \% AFW: ses ton } \\
\text { Current }\end{array}$ & $\begin{array}{r}6.0 \\
26.4\end{array}$ & $\begin{array}{r}6.7 \\
25.2\end{array}$ & $\begin{array}{r}3.5 \\
18.7\end{array}$ & $\begin{array}{r}4.1 \\
19.1\end{array}$ & $\begin{array}{l}6.0 \\
4.0\end{array}$ & $\begin{array}{l}3.0 \\
4.0\end{array}$ \\
\hline Jun & & & & & & \\
\hline $\begin{array}{l}\text { Zooplankton } \\
\text { Plants } \\
\text { Benthos } \\
\text { Mysis } \\
\text { Fish } \\
\text { Seston } \\
\text { Detritus } \\
\text { Total }\end{array}$ & $\begin{array}{r}2,729.7 \\
1,968.0 \\
66.3 \\
2.1 \\
1.5 \\
38,846.7 \\
36,117.0 \\
40,882.5\end{array}$ & $\begin{array}{r}1,408.8 \\
37.8 \\
68.0 \\
11.0 \\
1.9 \\
5,973.2 \\
4,564.4 \\
6,080.9\end{array}$ & $\begin{array}{r}1,260.1 \\
23.1 \\
5.3 \\
0.0 \\
5.6 \\
2,549.2 \\
1,289.1 \\
2,583.2\end{array}$ & $\begin{array}{r}839.8 \\
21.2 \\
231.4 \\
13.0 \\
15.1 \\
1,207.0 \\
367.2 \\
1,474.7\end{array}$ & $\begin{array}{r}15.2 \\
27.3 \\
9.6 \\
0.0 \\
0.1 \\
7,742.1 \\
7,726.3 \\
7,763.3\end{array}$ & $\begin{array}{r}65.2 \\
40.1 \\
141.4 \\
0.0 \\
0.2 \\
15,419.2 \\
15,354.0 \\
15,600.9\end{array}$ \\
\hline $\begin{array}{l}\text { \% AFW: ses ton } \\
\text { Current } \\
\text { Weather }\end{array}$ & $\begin{array}{r}32.5 \\
38.4 \\
2.0\end{array}$ & $\begin{array}{r}21.1 \\
30.1 \\
1.0\end{array}$ & $\begin{array}{r}25.9 \\
15.5 \\
1.3\end{array}$ & $\begin{array}{r}23.3 \\
12.2 \\
1.0\end{array}$ & $\begin{array}{r}25.2 \\
18.9 \\
2.2\end{array}$ & $\begin{array}{r}28.5 \\
15.4 \\
2.0\end{array}$ \\
\hline
\end{tabular}


mysids in night over day collections was associated with vertical migration from the river floor.

River load concentration varied between No. 2-mesh $(355 \mu \mathrm{m})$ and No. 10-mesh $(153 \mu \mathrm{m})$ net collections (Table 4l). Zooplankton and seston concentrations were higher in No. 10-mesh and No. 2mesh-net collections at Frechette Point and Lake Nicolet; total river load concentrations also were higher in the No. 10-mesh net collections. These differences were statistically significant ( $p$ $=0.05 ;$ Mann-Whitney U-test). At Point aux Frenes, zooplankton dry-weight concentrations (but not abundance) were significantly greater in the coarser No. 2-mesh net collections; mean size of zooplankton also was larger in the No. 2-mesh net collections averaging $11.2 \mu \mathrm{g}$ versus $8.0 \mathrm{\mu g}$ for the No. 10 -mesh net. Seston and total river load concentrations were significantly higher in the No. 2-mesh than the No. 10-mesh net collections at this location. The reason for this difference has no ready explanation. Differences in detritus, plant, benthos, and mysid concentrations between No. 2-mesh and No. 10-mesh net collections were not statistically significant among sites. This suggests that these particulates were sufficiently large to be efficiently captured by the No. 2-mesh net. Although \&AFW:seston tended to be higher in No. 10-mesh than No. 2-mesh net collections, differences were not statistically significant.

\section{River Load During Spring--}

With the exception of mysids and $8 A F W:$ seston, differences in June river load composition were significantly $(p=0.05$; Kruskal-Wallis test) different among Frechette Point, Lake Nicolet, and Point aux Frenes. Total load (dry weight, No. 2mesh net) averaged $23,147 \mathrm{mg} / 1,000 \mathrm{~m}^{3}$ at Frechette Point, 2,024 $\mathrm{mg} / 1,000 \mathrm{~m}^{3}$ at Lahe Nicolet, and $1.1,729 \mathrm{mg} / 1,000 \mathrm{~m}^{3}$ at Point aux Frenes (Table 37; Appendixes 155-174, 201-228, 242-261). Seston was the major component of the river load at all three stations.

The river apparently carried a greater mineral load in June than in February-March (Tables 36, 37) as \%AFW:seston was considerably higher in June (25.6\%) than in February-March $(4.8 \%)$. This increase may have been associated with increased inflows from small streams and rivers. It may also have been associated with the disappearance of the protective ice cover which had served to limit wind-induced wave activity and sediment resuspension.

At Frechette Point, zooplankton was a minor component of the June river load averaging $8.9 \%$ versus $50.5 \%$ in February-March (Table 37). This decrease in percent composition was due mainly to the large increase in detritus between winter $(3,225 \mathrm{mg} / 1,000$ $\left.\mathrm{m}^{3}\right)$ and spring $\left(20,037 \mathrm{mg} / 1,000 \mathrm{~m}^{3}\right)$ sampling. Plants were more 
Table 41. Mean concentration ( $\mathrm{mg} / \mathrm{dry}$ weight/1,000 $\mathrm{m}^{3}$ ) of the various components of river drift in No. 2-mesh and No. 10-mesh net collections, 26 February3 March 1985 and 2-13 June 1985. Also given is the percentage of dry weight that is ash-free dry weight (AFW). Mysis weights are included in the total benthos weight. Detritus weights are the difference between seston and zooplankton weights.

\begin{tabular}{|c|c|c|c|c|c|c|}
\hline \multirow[b]{2}{*}{ Parameter } & \multicolumn{2}{|c|}{ Frechette Point } & \multicolumn{2}{|c|}{ Lake Nicolet } & \multicolumn{2}{|c|}{ Point aux Frenes } \\
\hline & No. 2 & No. 10 & No. 2 & No. 10 & No. 2 & No. 10 \\
\hline $\begin{array}{l}\text { Feb-Mar } \\
\text { Zooplankton } \\
\text { Plants } \\
\text { Benthos } \\
\text { Mysis } \\
\text { Fish } \\
\text { Seston } \\
\text { Detritus } \\
\text { Total }\end{array}$ & $\begin{array}{r}3,863.0 \\
55.2 \\
86.1 \\
6.7 \\
0.0 \\
8,353.4 \\
4,490.4 \\
8,494.7\end{array}$ & $\begin{array}{r}8,856.9 \\
89.6 \\
44.7 \\
0.4 \\
0.0 \\
13,034.9 \\
4,178.0 \\
13,168.3\end{array}$ & $\begin{array}{r}4,375.5 \\
3.3 \\
22.9 \\
20.2 \\
0.0 \\
6,631.5 \\
2,238.0 \\
6,657.7\end{array}$ & $\begin{array}{r}7,170.1 \\
3.4 \\
18.3 \\
14.9 \\
0.0 \\
10,530.5 \\
3,360.9 \\
10,552.2\end{array}$ & $\begin{array}{r}2,345.0 \\
1.2 \\
4.3 \\
2.8 \\
0.0 \\
3,439.6 \\
1,094.6 \\
3,445.0\end{array}$ & $\begin{array}{r}1,458.5 \\
0.6 \\
4.0 \\
0.7 \\
0.0 \\
1,888.7 \\
430.2 \\
1,893.3\end{array}$ \\
\hline \% AFW: seston & 7.3 & 14.2 & 4.4 & 4.9 & 5.0 & 10.2 \\
\hline Jun & & & & & & \\
\hline $\begin{array}{l}\text { Zooplankton } \\
\text { Plants } \\
\text { Benthos } \\
\text { Mysis }\end{array}$ & $\begin{array}{r}2,905.9 \\
2,705.8 \\
76.4 \\
3.9\end{array}$ & $\begin{array}{r}3,770.4 \\
79.3 \\
53.0 \\
0.1\end{array}$ & $\begin{array}{r}849.2 \\
53.3 \\
146.6 \\
20.8\end{array}$ & $\begin{array}{r}1,035.5 \\
69.0 \\
47.2 \\
2.1\end{array}$ & $\begin{array}{r}23.1 \\
32.3 \\
9.3 \\
0.0\end{array}$ & $\begin{array}{r}585.0 \\
43.3 \\
28.5 \\
0.4\end{array}$ \\
\hline$\overline{\text { F1sh }}$ & 3.8 & 2.1 & 6.1 & 13.1 & 0.2 & 0.6 \\
\hline $\begin{array}{l}\text { Seston } \\
\text { Detritus } \\
\text { Total }\end{array}$ & $\begin{array}{l}18,722.7 \\
15,816.8 \\
21,508.7\end{array}$ & $\begin{array}{r}12,857.6 \\
8,883.2 \\
12,992.0\end{array}$ & $\begin{array}{r}1,258.6 \\
400.4 \\
1,464.6\end{array}$ & $\begin{array}{l}3,023.1 \\
1,987.6 \\
3,152.4\end{array}$ & $\begin{array}{l}1,299.8 \\
1,276.7 \\
1,341.6\end{array}$ & $\begin{array}{l}37,625.0 \\
37,040.0 \\
37,697.4\end{array}$ \\
\hline$\%$ AFW: ses ton & 26.4 & 43.2 & 22.8 & 45.0 & 27.9 & 23.7 \\
\hline
\end{tabular}


abundant in June $\left(984 \mathrm{mg} / 1,000 \mathrm{~m}^{3}\right)$ than February $(73 \mathrm{mg} / 1,000$ $\mathrm{m}^{3}$ ), averaging $22.2 \%$ of the total river load versus $1.1 \%$ in winter; as in February-March, the greatest biomass of plants was observed at Frechette point. Benthos and $f$ ish, while more abundant in spring than in winter, continued to be minor components of river load. February-June statistical comparisons Mann-Whitney $U$-test) were significant $(p=0.05)$ for all categories except mysids, seston, and total river load.

At Lake Nicolet, zooplankton was a smaller contributor to total river load in June (51.8\%) than February (72.4\%) (Table 37). Detritus accounted for an average of $40.6 \%$ of the river load in June versus $27.0 \%$ in February; however, detritus concentrations were lower in June than in February. Plants, benthos (excluding mysids), and $f$ ish all increased in biomass between February and June but were relatively minor components of river load. All winter-spring comparisons were statistically significant ( $p=0.05$; Mann-Whitney U-test).

At Point aux Frenes, total river load concentration increased markedly between February-March $\left(2,687 \mathrm{mg} / 1,000 \mathrm{~m}^{3}\right)$ and June $\left(11,728 \mathrm{mg} / 1,000 \mathrm{~m}^{3}\right)$ (Table 37$)$, although these differences were not statistically significant $(p=0.05$; Mann-Whitney Utest). Zooplankton accounted for a very small component of the river load, averaging 0.38 in June versus $76.5 \%$ in winter. This low contribution was due to a marked decline in zooplankton abundance and mean size between winter and June while detritus concentrations increased 18-fold. Biomass of plants, benthos, and $f$ ish increased between winter and spring. With the exception of total river load, all winter-spring statistical comparisons were significant ( $p=0.05$; Mann-Whitney U-test).

As in February-March, there were differences in river load concentration and composition between channel and shallow stations (Table 38). At Frechette Point, zooplankton and mysid biomass was significantly ( $p=0.05$; Mann-Whitney U-test) greater in channel station samples, while benthic biomass was significantly greater in shallow station samples; differences in oAFW:seston also were significant. At Lake Nicolet, zooplankton biomass was significantly greater in channel station samples, while seston biomass was greater at shallow stations. Although detritus concentrations were higher at shallow stations, differences were not significant. At Point aux Frenes, zooplankton, detritus, and seston biomass was greater in shallow station samples, while fish were more abundant in the channel.

Although total river load concentration varied with depth strata at Frechette Point (Table 39), such differences were not significant ( $p=0.05$; Kruskal-Wallis test for three strata comparisons and Mann-Whitney U-test for two-depth comparisons). Differences in zooplankton and detritus biomass (which increased 
with depth) and benthic biomass (which decreased with depth) were significant. At Lake Nicolet, zooplankton, seston, fish, and total river load biomass varied significantly with depth strata; maximum biomass was observed at mid-depth strata. At Point aux Frenes, benthos, seston, fish, detritus, and total biomass varied significantly among the three depth strata with lowest biomass observed in surface strata. Mid-depth versus bottom comparisons were significant only for benthos.

There were significant differences in river load concentration and composition between day and night collections. At Frechette Point, zooplankton, plant, seston, detritus, and total biomass densities were higher during day collections than night collections (Table 40); these differences were significant $(p=0.05 ;$ Mann-Whitney U-test). Current velocities also were significantly higher during the day $(38.4 \mathrm{~cm} / \mathrm{s}$ versus $30.1 \mathrm{~cm} / \mathrm{s})$. Day-night differences in weather conditions were statistically significant. The weather was windy during the day but calm at night. Differences in benthos, mysid, and $f$ ish biomass between day and night sampling were not significant.

At Lake Nicolet, differences in zooplankton, seston, detritus, and total biomass between day and night sampling (Table $40)$ were significant $(p=0.05$; Mann-Whitney U-test). In all instances, biomass was higher during the day than during the night. Such differences may have been related to physical factors such as current velocity and resuspension. Current velocities were higher during the day $(15.5 \mathrm{~cm} / \mathrm{s}$ versus $12.2 \mathrm{~cm} /$ s) and the weather was slightly more windy ( 1.3 versus 1.0$)$; these differences were significant. Benthos, including mysids, and $f$ ish were significantly more abundant at night. This suggests that, in contrast to Frechette Point, some components of the benthic community made diel migrations between the river floor and water column.

At Point aux Frenes, zooplankton and benthos were more abundant at night than during the day, and differences were significant ( $p=0.05$; Mann-Whitney U-test). While the mean sizes of zooplankton collected during day and night collections were similar at Frechette Point $(12.0 \mu \mathrm{g}$ and $11.9 \mu \mathrm{g})$ and Lake $\mathrm{Nicolet}(11.7 \mathrm{\mu g}$ and $11.7 \mathrm{\mu g})$, there were large differences in the mean size of zooplankton in day $(4.9 \mu \mathrm{g})$ and night $(1.7 \mu \mathrm{g})$ collections at Point aux Frenes (Table 35 ). This suggests that the increased biomass of zooplankton at night at Point aux Frenes was associated with the vertical migration of small epibenthic zooplankton, i.e., immature Cyclops spp. and Mesocyclops edax. As zooplankton and benthos were minor components of the river load at Point aux Frenes, day and night differences in total river load were not significant. Detritus concentrations were not significantly different between day and night collections despite higher current velocities during daylight hours $(18.9 \mathrm{~cm} /$ 
s versus $15.4 \mathrm{~cm} / \mathrm{s}$ ). Although it was windier during the day than night $(2.2$ versus 2.0$)$, these differences were not significant. This suggests that during calm weather (e.g., at Frechette Point), current velocity has a significant effect on river load. However, as the weather becomes more windy, small differences in current velocity may not have as major an effect on river load as increased wave activity resulting from the windier conditions.

River load estimates differed between No. 2-mesh and No. 10mesh net collections (Table 41). At Frechette Point, differences were significant only for $f$ ish, total biomass, and $8 A F W:$ seston; estimates were higher in No. 2-mesh than No. 10-mesh net samples. There is no ready explanation for these higher biomass estimates from the No. 2-mesh net.

At Lake Nicolet, net comparisons were significant for seston, fish, detritus, total biomass, and \&AFW:seston; the No. 10-mesh net collected the greater biomass (Table 41 ). Although zooplankton were significantly more abundant in No. 10mesh net collections than No. 2-mesh net collections, biomass was not significantly different. This is because, although small zooplankton were collected in greater numbers in the No. 10-mesh net collection, they apparently did not contribute substantially to biomass.

At Point aux Frenes, net comparisons were significant only for zooplankton, seston, and total biomass; the No. 10-mesh net collected the greater biomass (Table 41). Although detritus concentrations were higher in the No. 10-mesh net collections, these differences were not statistically significant $(p=0.05$; Mann-Whitney U-test).

\section{FISH LARVAE}

Lake Herring

Lake herring (Coregonus artedii) larvae (total number collected $=281$ ) were collected during all 6 wk of the study and at all seven stations. The total number collected per week (same effort) (Fig. 14) started at 52, peaked at 80 , and then declined to 14 larvae in the last week.

Densities of larvae were generally greatest during the first 3 wk and tapered off during the last 3 wk of May (Fig. 15, Table 42). Mean densities varied from 0 to 1,451 larvae $/ 1,000 \mathrm{~m}^{3}$. Maximum densities usually occurred at the 1-m depth, but occasionally densities were greater at $2 \mathrm{~m}$. Neither depth had a consistently greater density of larval lake herring over the 6 wk. In the channel, densities were very low with a maximum of 


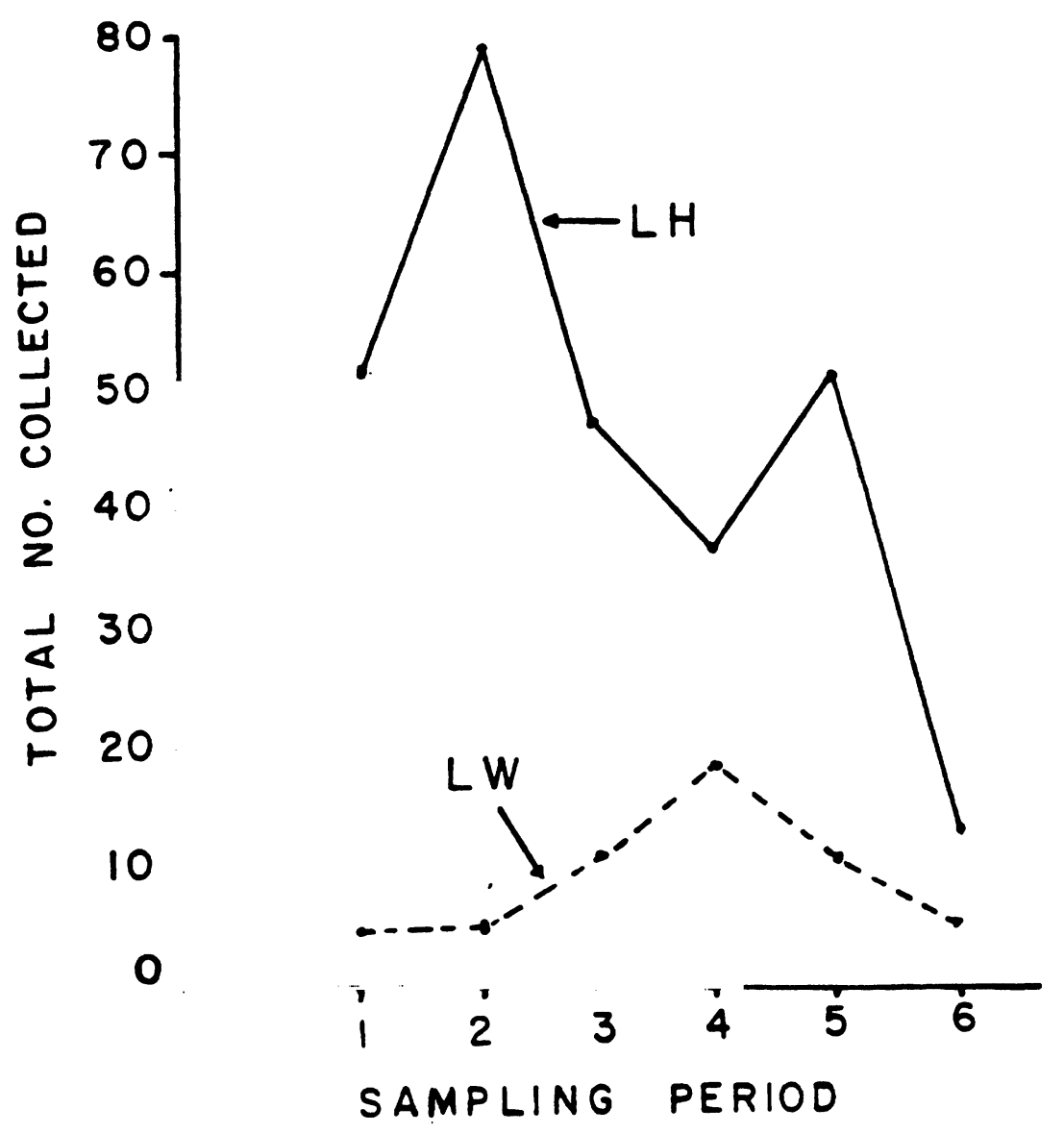

Figure 14. Total number (NO.) of lake herring (LH) and lake whitefish (LW) larvae collected over 6 wh ( 24 April to 30 May) in the St. Marys River, 1985. 


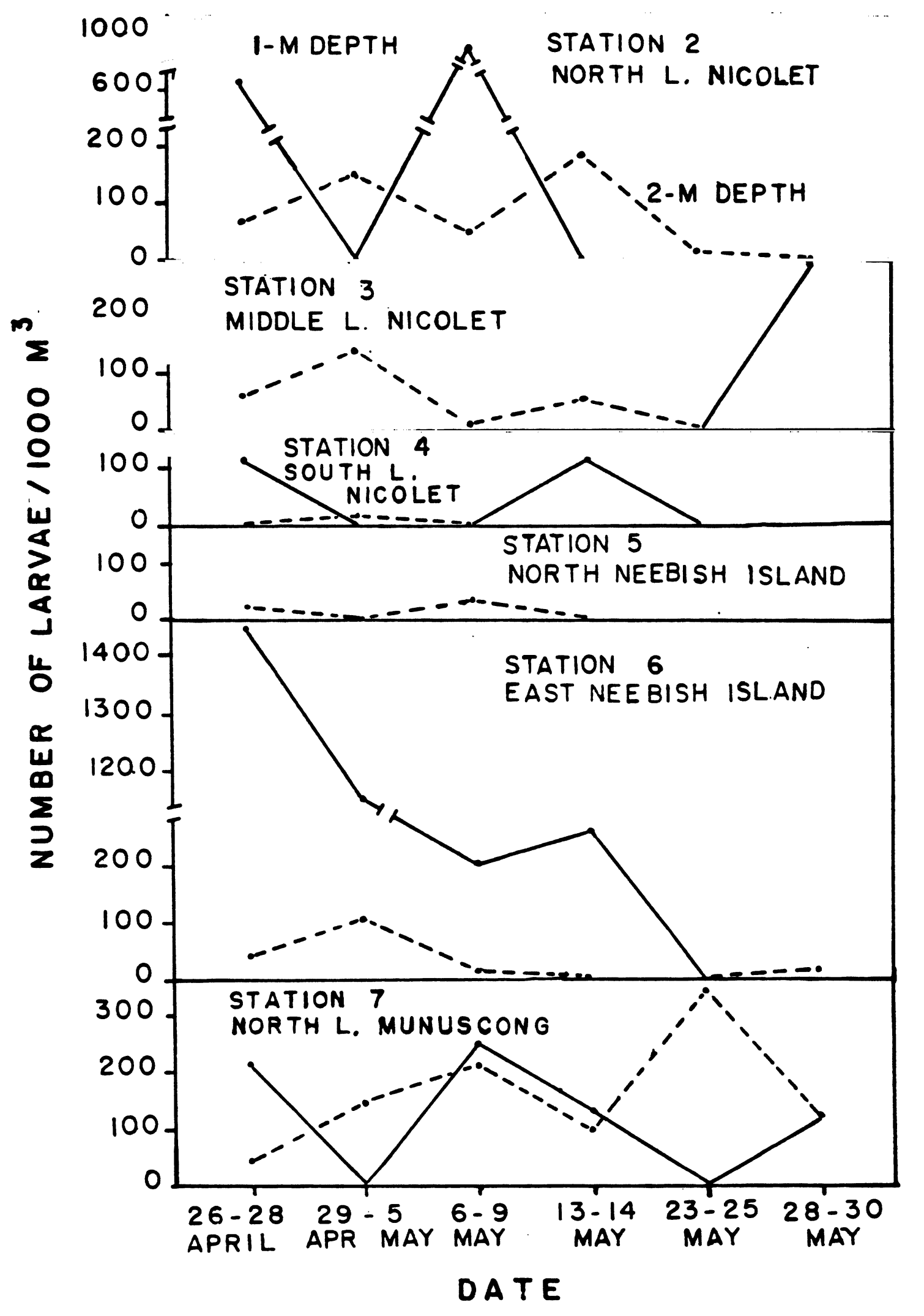

Figure 15. Density of lake herring larvae collected from 26 April to 30 May at six stations in the St. Marys River. 


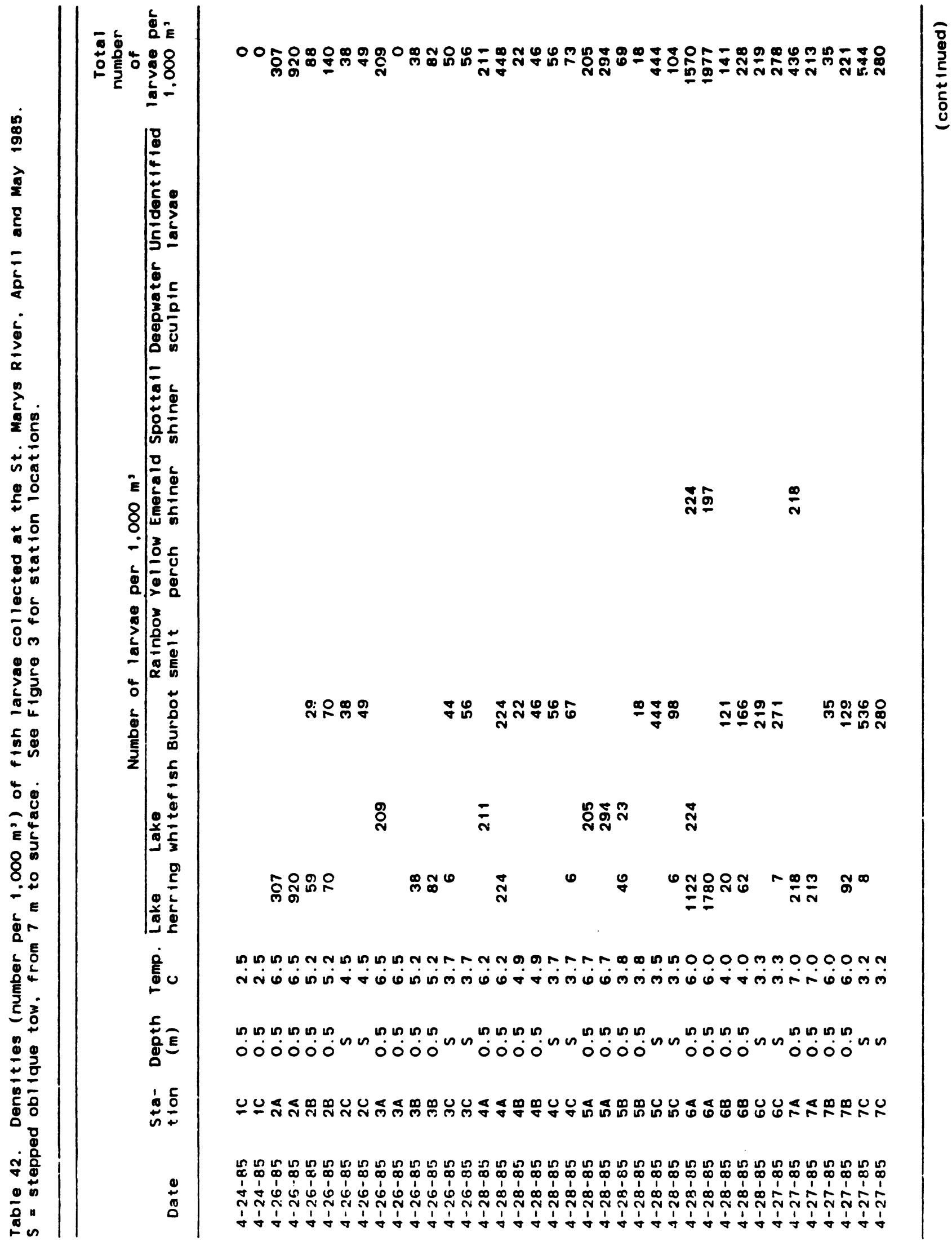




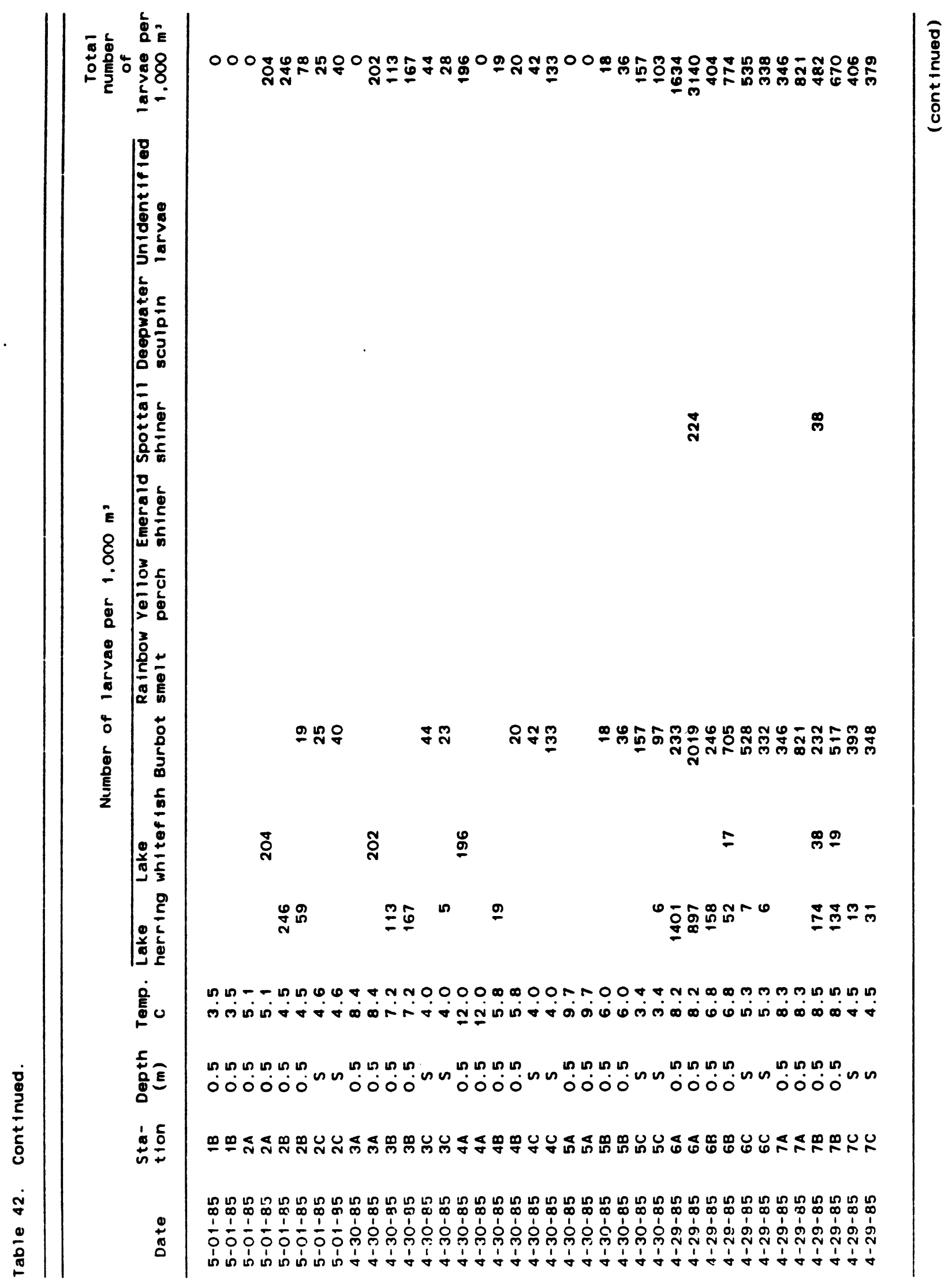




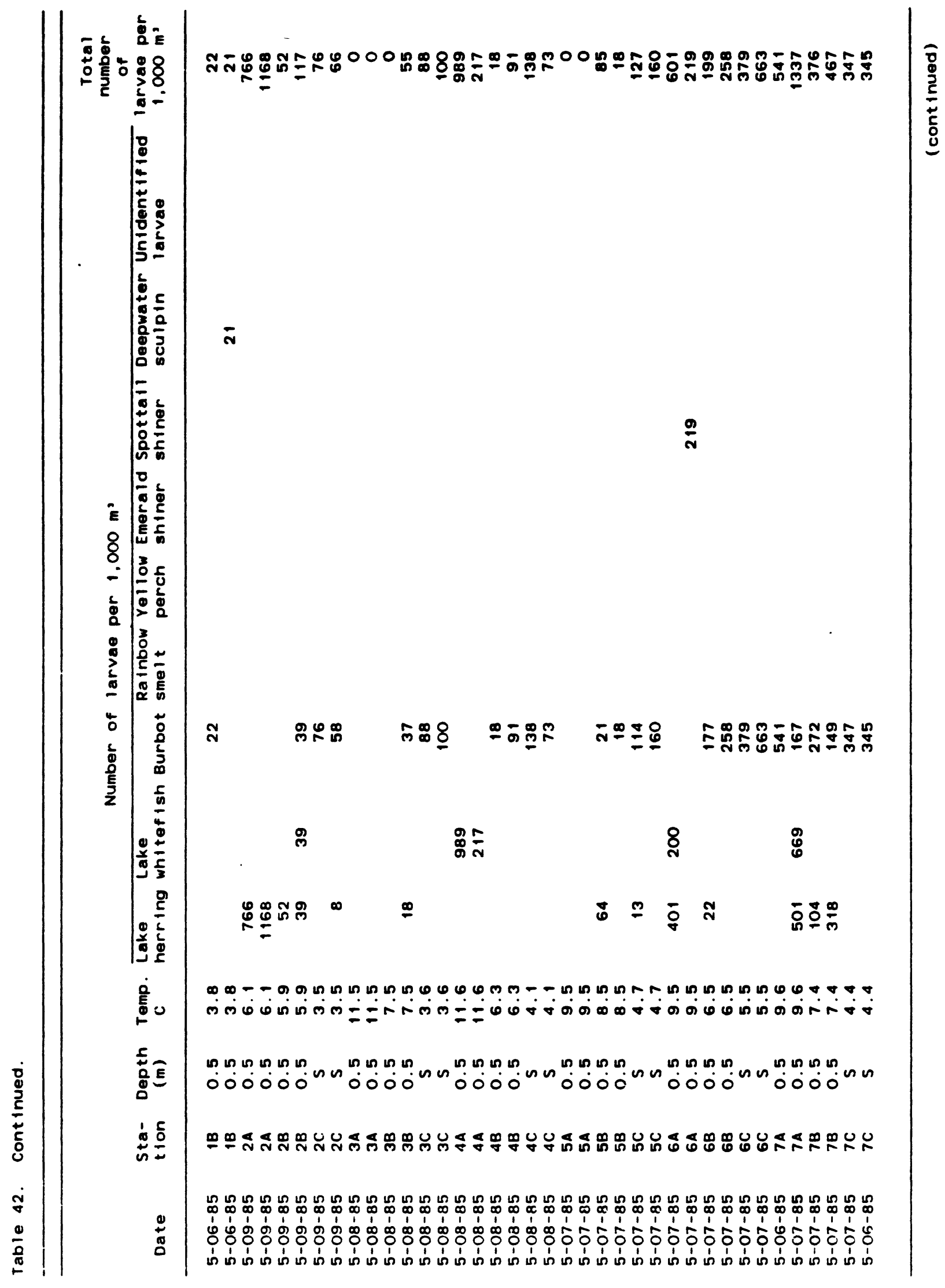




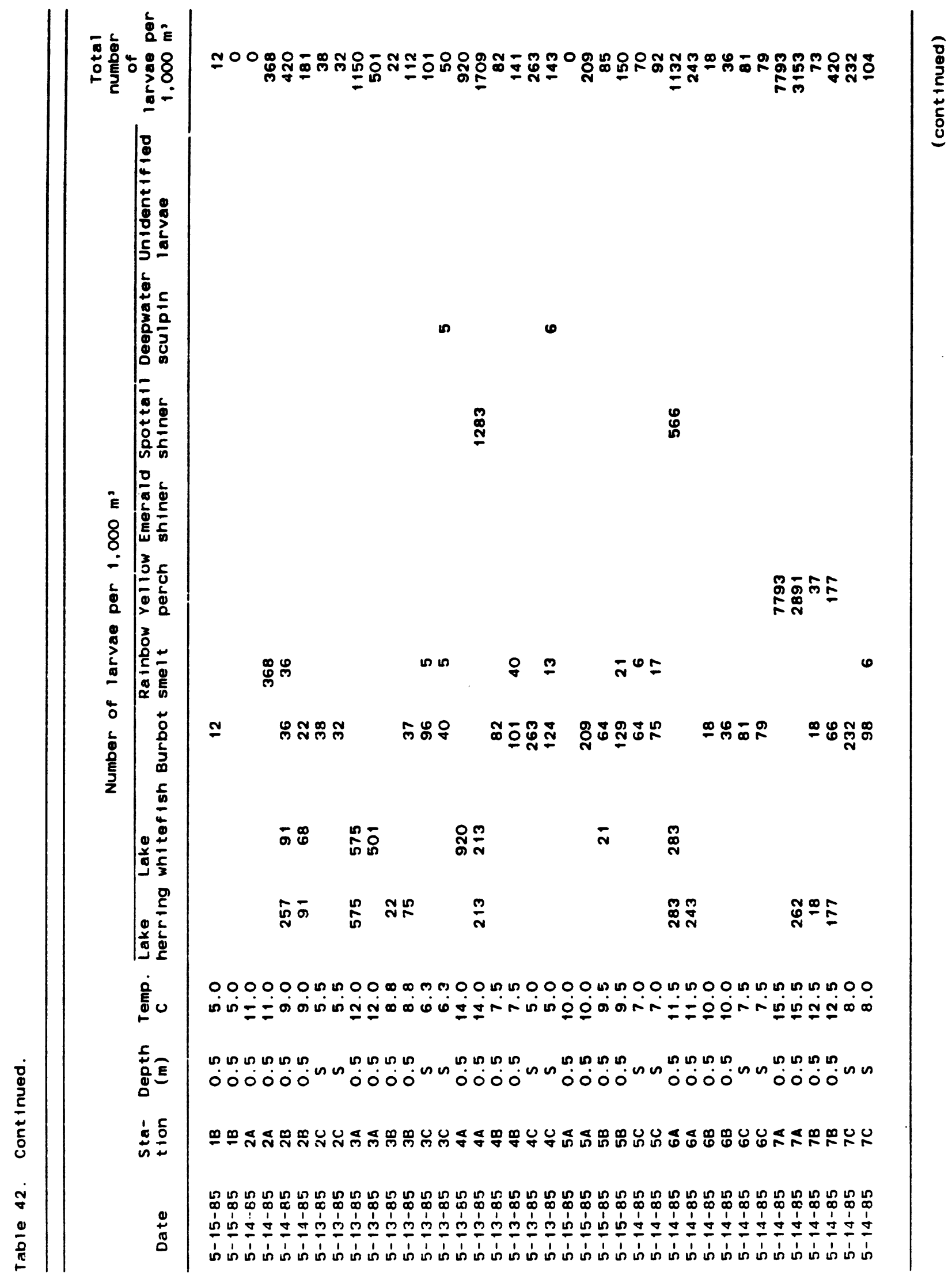




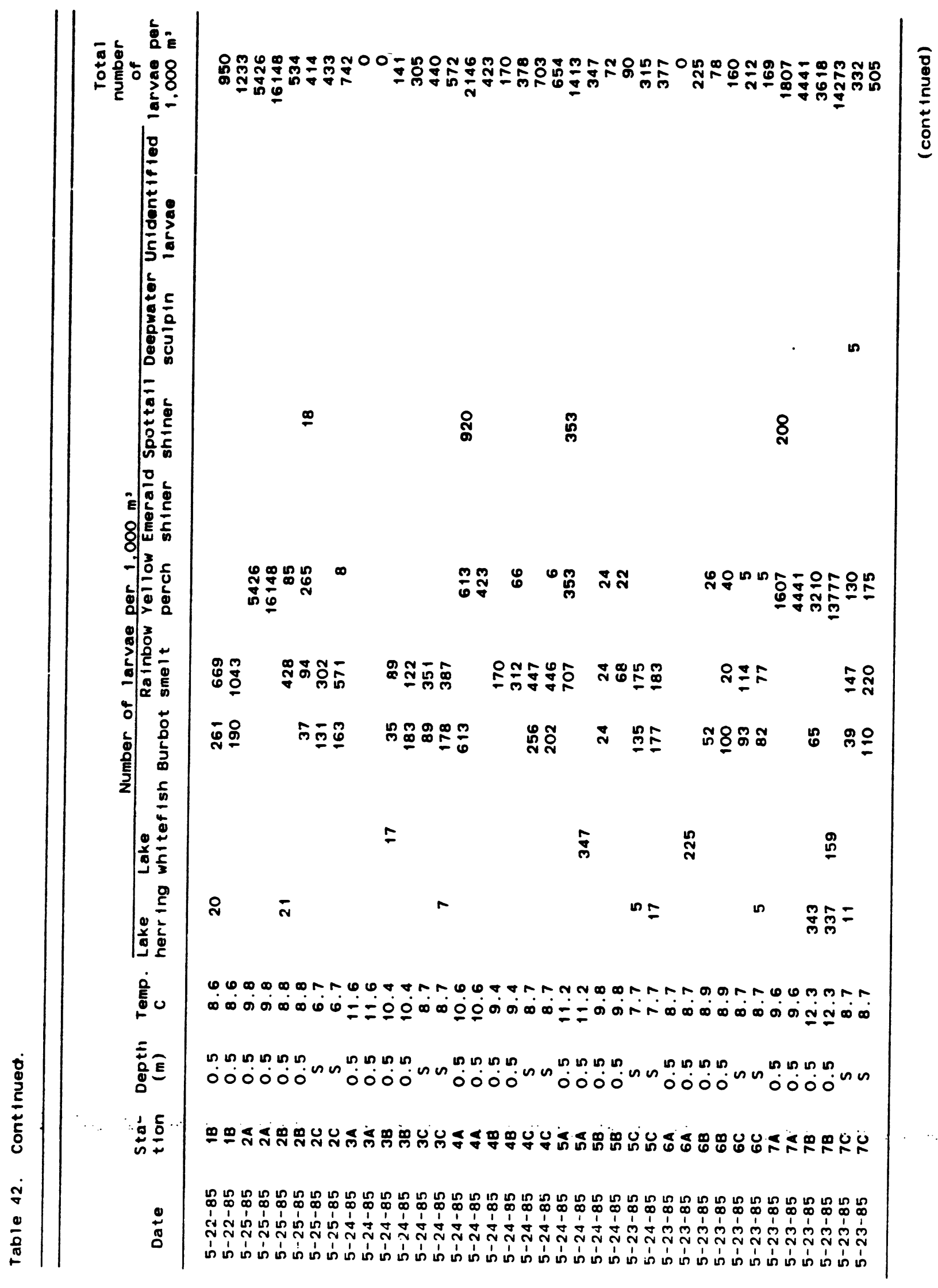




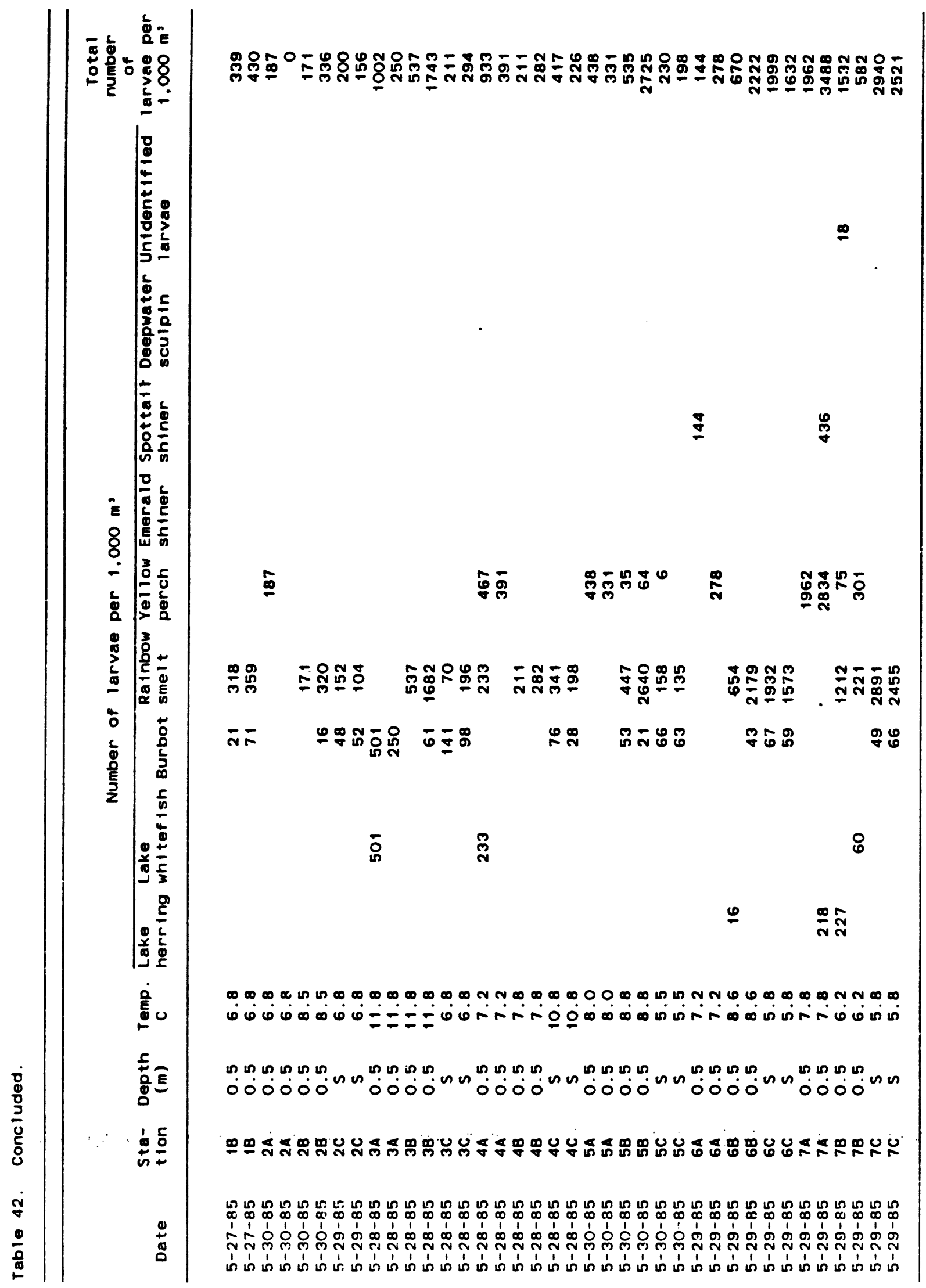


only 17 larvae/1,000 $\mathrm{m}^{3}$. Only $9 \%$ of the larvae were collected in the channel.

Although larval lake herring were collected at all seven stations, occurrences and densities varied considerably. Only two larvae were collected in the Edison Hydropower Canal station 1. At the other six stations, lowest densities and most sporadic occurrences were at stations 3,4 , and 5 (Fig. 15). During the first 3 sampling wk, larval $f$ ish densities were especially high at the $1-\mathrm{m}$ depth at the north Lake Nicolet and east Neebish Island stations. Lower densities occurred at station 7 - north Lake Munuscong, but some larval lake herring were present throughout the $6 \mathrm{wk}$. Among stations, densities of larval $f$ ish were generally highest at the 2-m depth.

Average size of larval lake herring increased from April to the end of May (Fig. 16). Since lake herring hatch at 8.5 to $12.8 \mathrm{~mm}$ (Auer 1982), the presence of a few 10.2- to 12.0-mm larvae suggests some hatching occurred during the fourth week of May. Based on larval $f$ ish sizes, hatching was completed by the last week of May.

The length-frequency distribution of lake herring larvae at all stations below the Sault Power Canal (Fig. 17) showed larvae we collected ranged from about 8 to $25 \mathrm{~mm}$. Modes were observed at about 11 and $17 \mathrm{~mm}$.

The percentage of the lake herring collected that were nonyolk-sac larvae increased almost linearly over the course of the study (Fig. 18) from 0 to $2 \%$ in the first 2 wk, up to $100 \%$ during the last week. Therefore, the decline in the total number of larvae that we collected, from 52 in week 1 to 14 in week 6 , is partially attributable to net avoidance as well as natural mortality as the larvae grow older. Overall, $61 \%$ of all lake herring larvae collected were yolk-sac larvae and $39 \%$ were nonyolk-sac larvae.

\section{Lake Whitefish}

Lake whitefish (Coregonus clupeaformis) larvae were collected during all 6 wk of the study and at six of the seven stations. Larvae were not collected in the Edison Hydropower Canal. Sixty-two larvae were captured, making them less abundant than lake herring. The total number of lake whitefish larvae collected per week (Fig. 14) increased gradually from 6-7 in weeks 1 and 2 to a peak of 19 at week 4 , and then a gradual decline to 6 in the last week of May.

Densities were generally greatest during the first 2 wh of May (Fig. 19). Maximum densities occurred at 1-m depths and 


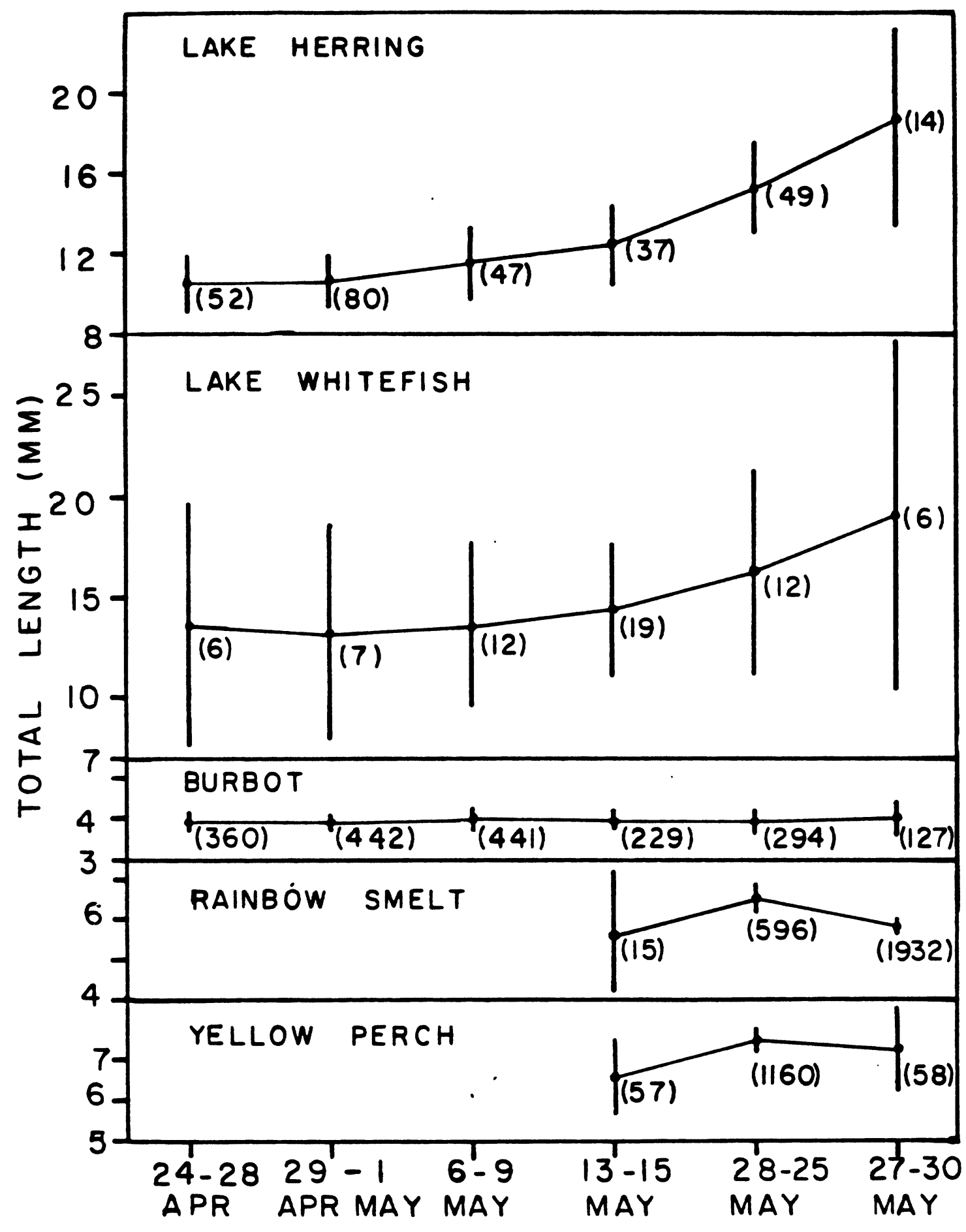

Figure 16. Mean total length and standard error (vertical lines) of five larval fish species collected in the St. Marys River, 1985. Total number collected is in parentheses. Larvae collected at Station 1 (Edison Hydropower Canal) are not included. 


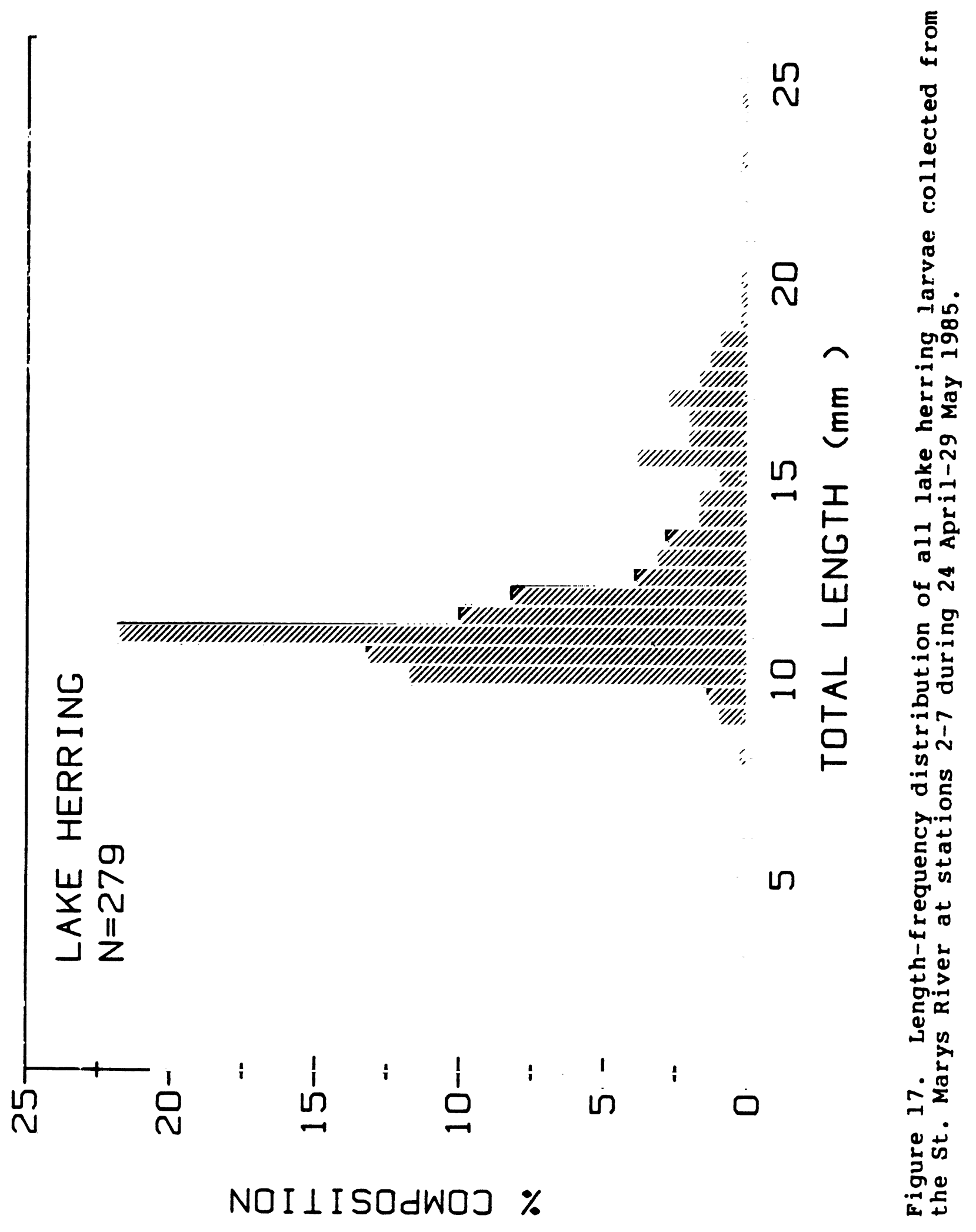




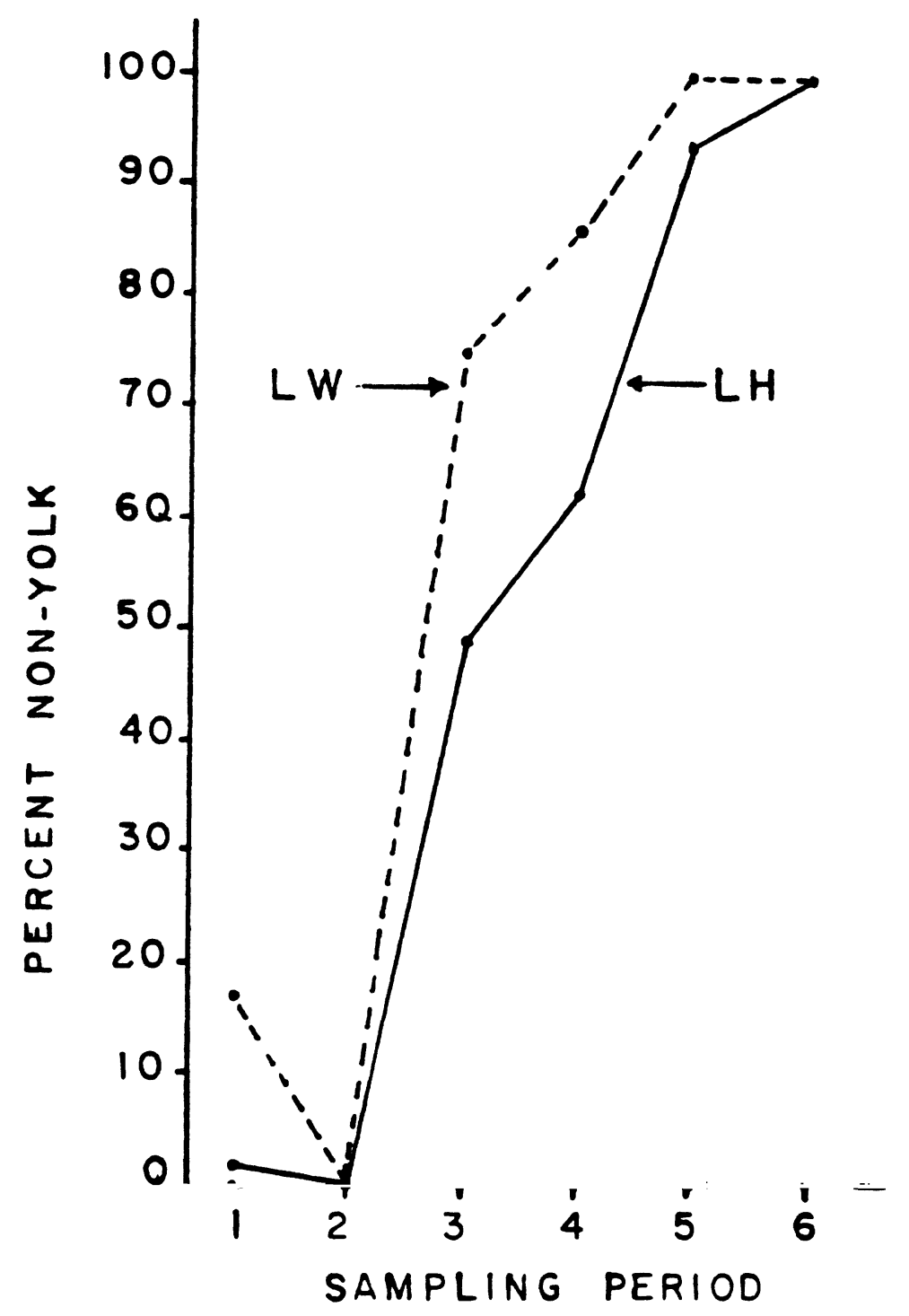

Figure 18. Percentage of non-yolk-sac lake herring (LH) and lake whitefish (LW) larvae collected over 6 wk (24 April to 30 May) in the St. Marys River, 1985. 


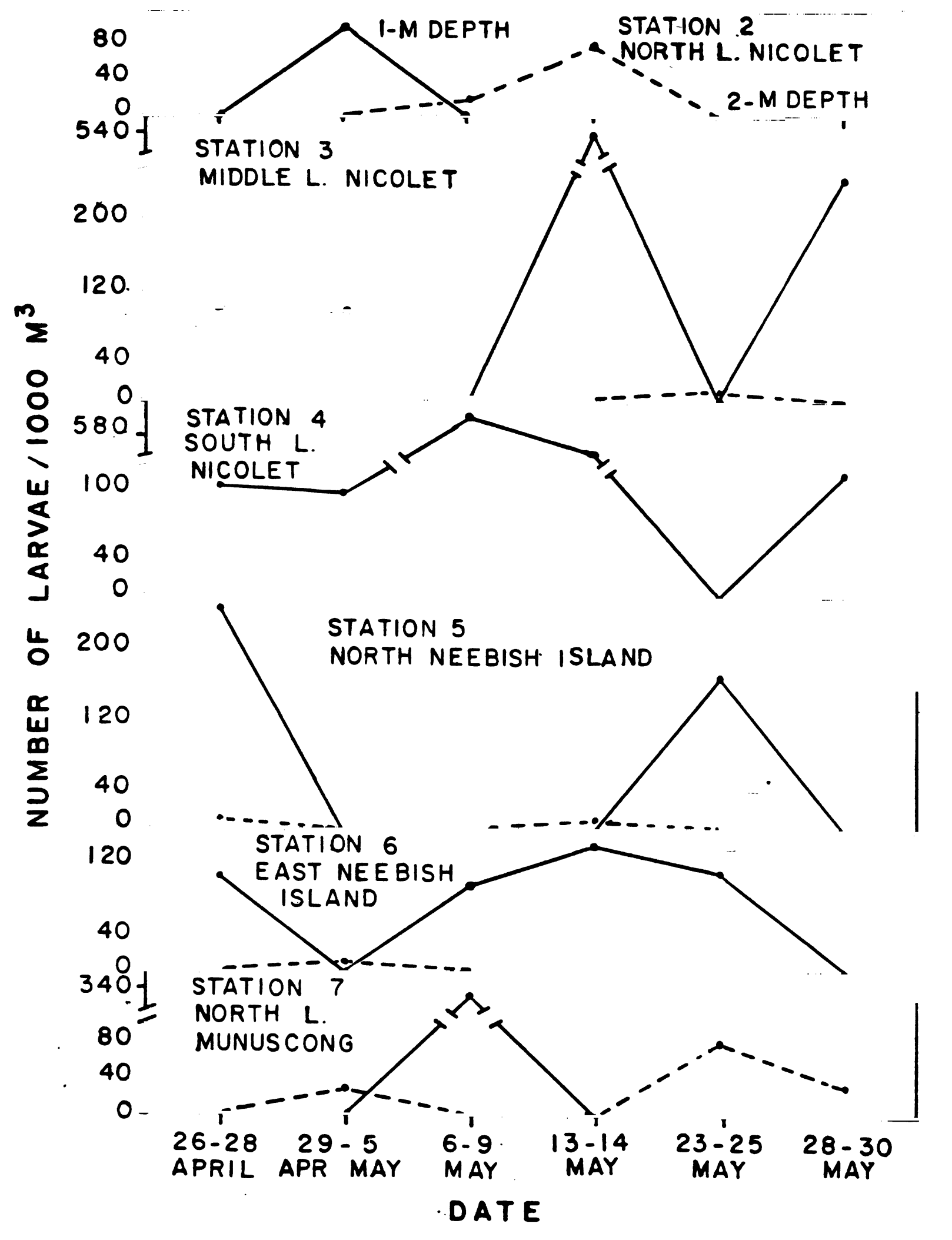

Figure 19. Density of lake whitefish larvae collected from 26 April to 30 May at six stations in the St. Marys River. 
ranged from about 600 to 1,000 larvae $/ 1,000 \mathrm{~m}^{3}$. Few lake whitefish larvae were captured at $2 \mathrm{~m}$, and none were caught in the channel. Although present at the six stations, occurrences and densities varied considerably among stations. The middle and south Lake Nicolet stations produced the most larvae.

Average sizes of lake whitefish larvae increased from April to the end of May (Fig. 16). Hatching appeared to be over by mid-May as few smaller larvae were collected thereafter. Lake whitefish larvae collected ranged from about 12 to $23 \mathrm{~mm}$ (Fig. 20). There were modes at around $14 \mathrm{~mm}$ and $22 \mathrm{~mm}$; the latter group was collected during the last week of the study. Lake whitefish hatch at sizes of 8.8-15 mm (Auer 1982). The curve for the percentage of lake whitefish larvae that were nonyolk-sac (Fig. 18) followed the same pattern observed for lake herring. Percentages were low in weeks 1 and 2 and then increased dramatically to $100 \%$ in weeks 5 and 6 . Overall, unlike lake herring where the pattern was reversed, $29 \%$ of the lake whitefish larvae were yolk-sac larvae, while $71 \%$ were non-yolksac larvae.

\section{Burbot}

Burbot (Lota lota) larvae were collected during all 6 wh of the study and at all seven stations. We collected 1,893 larvae.

Larval burbot densities peaked during the first week of May at the two stations farthest downstream (Table 42). These two stations also produced the highest densities recorded $(1,126$ and 584 larvae/l,000 $\mathrm{m}^{3}$ ) and were overall the most productive for burbot larvae. Peak densities at the other five stations occurred from mid-May to the end of May. Liston et al. (1986) collected burbot larvae from April to July with peak numbers in May.

Burbot larvae were most consistently present in channel samples, however, occurrences were similar between the channel and the 2-m depth. At $1 \mathrm{~m}$, occurrences were sporadic, but peak densities occurred there at three stations. These patterns from the three sampling gear used in 1985 are very similar to the findings of Liston et al. (1986) for 1982 and 1983.

Mean size of burbot larvae changed only slightly over the 6 sampling wk, suggesting continual hatching and recruitment throughout late April and all of May (Figs. 16, 21). Liston et al. (1986) found little change in larval burbot sizes until late June-early July. These results suggest an extended hatching period for burbot in the St. Marys River. 


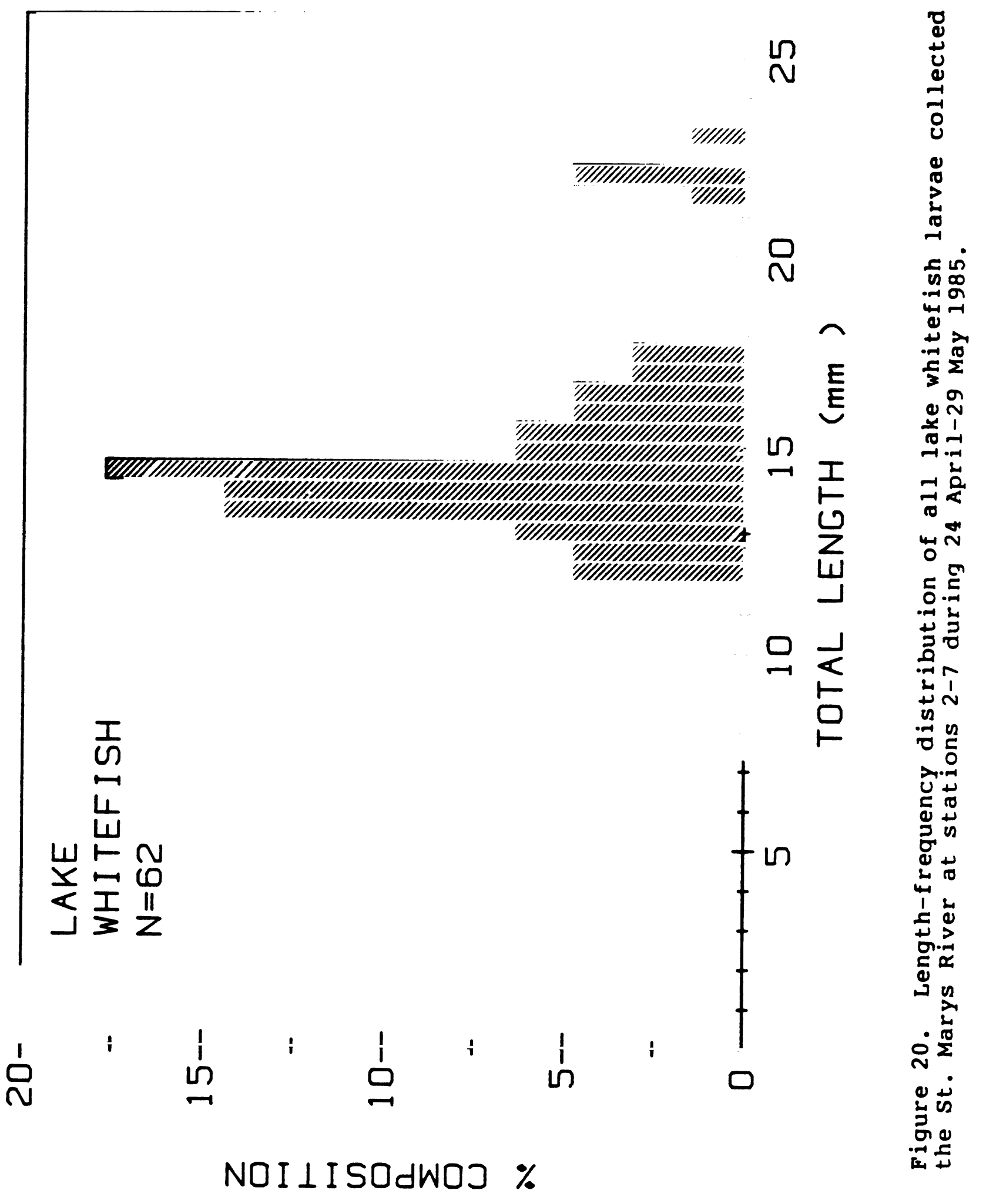




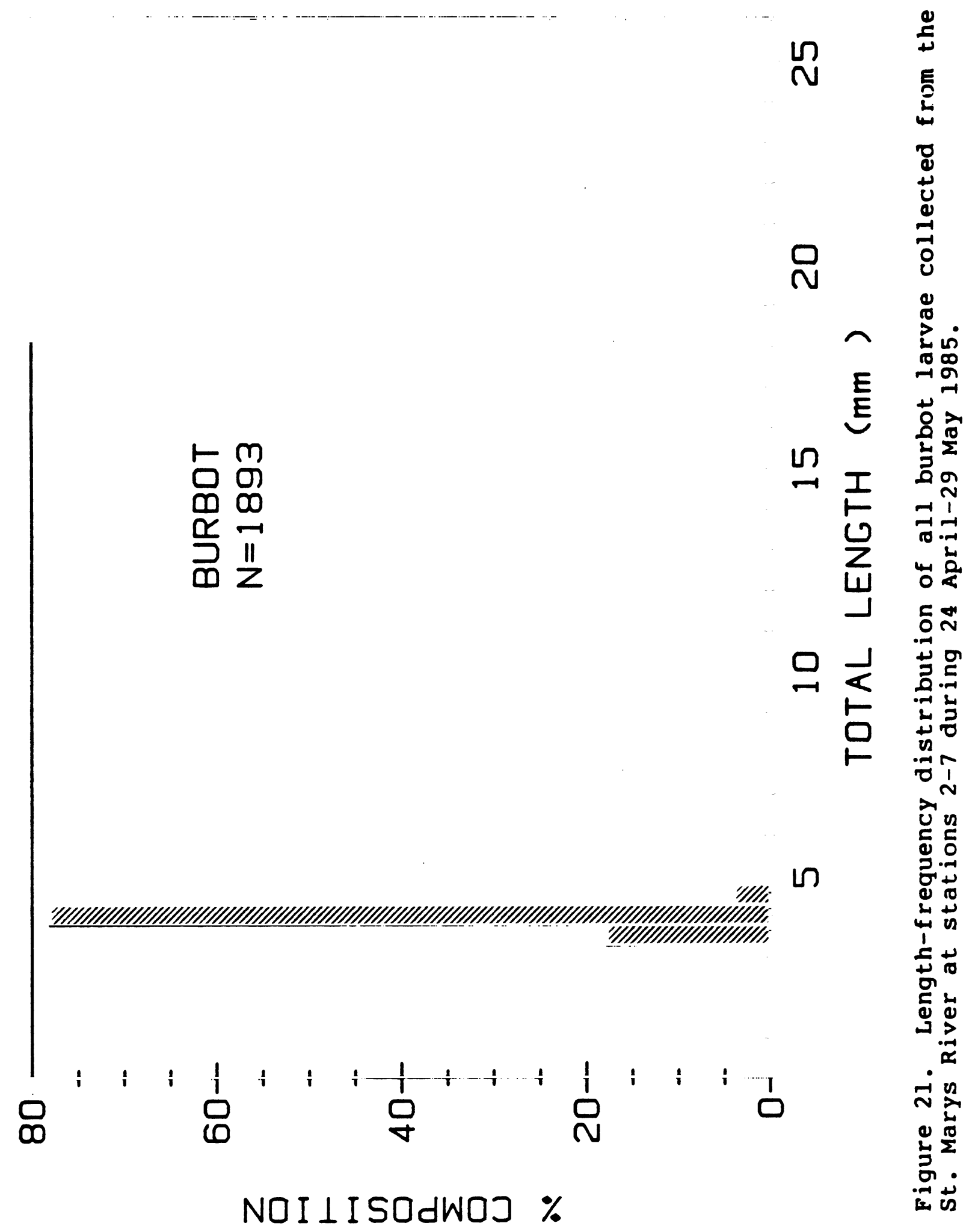


In summary, burbot larvae occur throughout the St. Marys River system. This suggests that burbot spawn throughout the system, but transport from localized areas cannot be dismissed. Burbot larvae are passively dispersed by currents shortly after hatching (Mansfield et al. 1983). The area around Neebish Island appears to be the most productive for burbot. Overall the river is moderate in burbot production, as the peak density of almost 1,500 larvae $/ 1,000 \mathrm{~m}^{3}$ (Table 43) was 16 times less than the 24,000 larvae/, $000 \mathrm{~m}^{3}$ found in Green Bay near Escanaba, Michigan (Mansfield et al. 1983).

\section{Rainbow Smelt}

Rainbow smelt (Osmerus mordax) larvae were not collected until 13 May (Table 42). Liston et al. $(1983,1986)$ also found initial occurrences of rainbow smelt in mid-May during 1981 and 1982, however in 1983 initial occurrence was on 5 May, suggesting earlier spawning. We found peak densities at most stations during the last week of May; however, these may not be seasonal peaks as Liston et al. (1986) found peak densities during the first 2 wh of June. Liston et al. (1986) also recorded a peak density of just over 20,000 larvae $/ 1,000 \mathrm{~m}^{3}$ which was more than 7 times greater than the peak density of just over 2,600 larvae/ $1,000 \mathrm{~m}^{3}$ that we found (Table 43).

We collected rainbow smelt larvae at all seven stations. In general, densities were greatest at the three stations around Neebish Island. Liston et al. (1986) also found greatest densities near the island. We found occurrences of larvae to be similar in the channel and at the 2-m depth. Occurrences were very sporadic at the $1-m$ depth. Greatest densities usually occurred in the channel, although at two stations maximum densities occurred at the 2-m depth. Overall these results agree with those of Liston et al. (1986) regarding the three depth zones.

Mean sizes of rainbow smelt larvae changed only slightly over the last 3 wh of May (Fig. 16). Lengths ranged from 4.5 to $10 \mathrm{~mm}$ with a mode at $5.5 \mathrm{~mm}$ (Fig. 22). This suggests continual hatching of fish larvae during this period. Liston et al. (1986) found larval rainbow smelt sizes did not increase noticeably until the end of June.

In simmary, rainbow smelt production is moderate to high in the St. Marys River. The waters around Neebish Island appear to be the most productive. 
Table 43. Maximum larval $\mathrm{f}$ ish densities (number/1,000 $\mathrm{m}^{2}$ ) of various $f$ ish species sampled in the $5 t$. Marys River.

\begin{tabular}{|c|c|c|c|c|}
\hline \multirow[b]{2}{*}{ Species } & \multicolumn{3}{|c|}{$\begin{array}{c}\text { Liston et al }(1983,1986) \\
\text { Liston and McNabb } 1986)\end{array}$} & \multirow{2}{*}{$\begin{array}{l}\text { Present } \\
\text { study } \\
1985 \\
\end{array}$} \\
\hline & 1981 & 1982 & 1983 & \\
\hline $\begin{array}{l}\text { Lake herring } \\
\text { Coregonus artedil }\end{array}$ & 340 & 4,970 & 2,030 & 1,450 \\
\hline $\begin{array}{l}\text { Lake whitefish } \\
\text { Coregonus clupeaformis }\end{array}$ & 2,380 & 770 & 4,300 & 603 \\
\hline $\begin{array}{l}\text { Burbot } \\
\text { Lota lota }\end{array}$ & 260 & 1,490 & 280 & 1,130 \\
\hline $\begin{array}{l}\text { Rainbow smelt } \\
\text { Osmerus mordax }\end{array}$ & 15,400 & 2,590 & 20,100 & 2,670 \\
\hline $\begin{array}{l}\text { Yellow perch } \\
\text { Perca flavescens }\end{array}$ & 14,500 & 35,400 & 14,500 & 10,800 \\
\hline
\end{tabular}

\section{Yellow Perch}

Yellow perch (Perca flavescens) larvae, like rainbow smelt, were not collected until mid-May (Table 42). Densities peaked during the last 2 weeks in May. A peak density of almost 11,000 larvae/ $1,000 \mathrm{~m}^{3}$ was recorded (Table 43 ). This may be a seasonal peak as Liston et al. (1986) found peak densities at the end of May.

We collected yellow perch larvae at $f$ ive of the seven stations. Larvae were absent from the Edison Hydropower Canal and at the middle Lake Nicolet stations. Lack of larvae in the canal may be due to later spawning in the Lake Superior area, as Liston et al. (1986) did not collect larvae there until late June and early July. Greatest densities occurred at the north Lake Munuscong station, although the overall peak density occurred at the north Lake Nicolet station. Liston et al. (1986) also found greatest densities in Lake Munuscong. Peak densities of 11,000 to 35,000 larvae/1,000 $\mathrm{m}^{3}$ (Table 43) indicate that the St. Marys River system, especially Lake Munuscong, is very productive for yellow perch.

Overall we found greatest densities at $1 \mathrm{~m}$, although the second highest density occurred at the 2-m depth. Densities were low and occurrences sporadic in the channel. These results agree with those of Liston et al. (1986) regarding depth zones. 


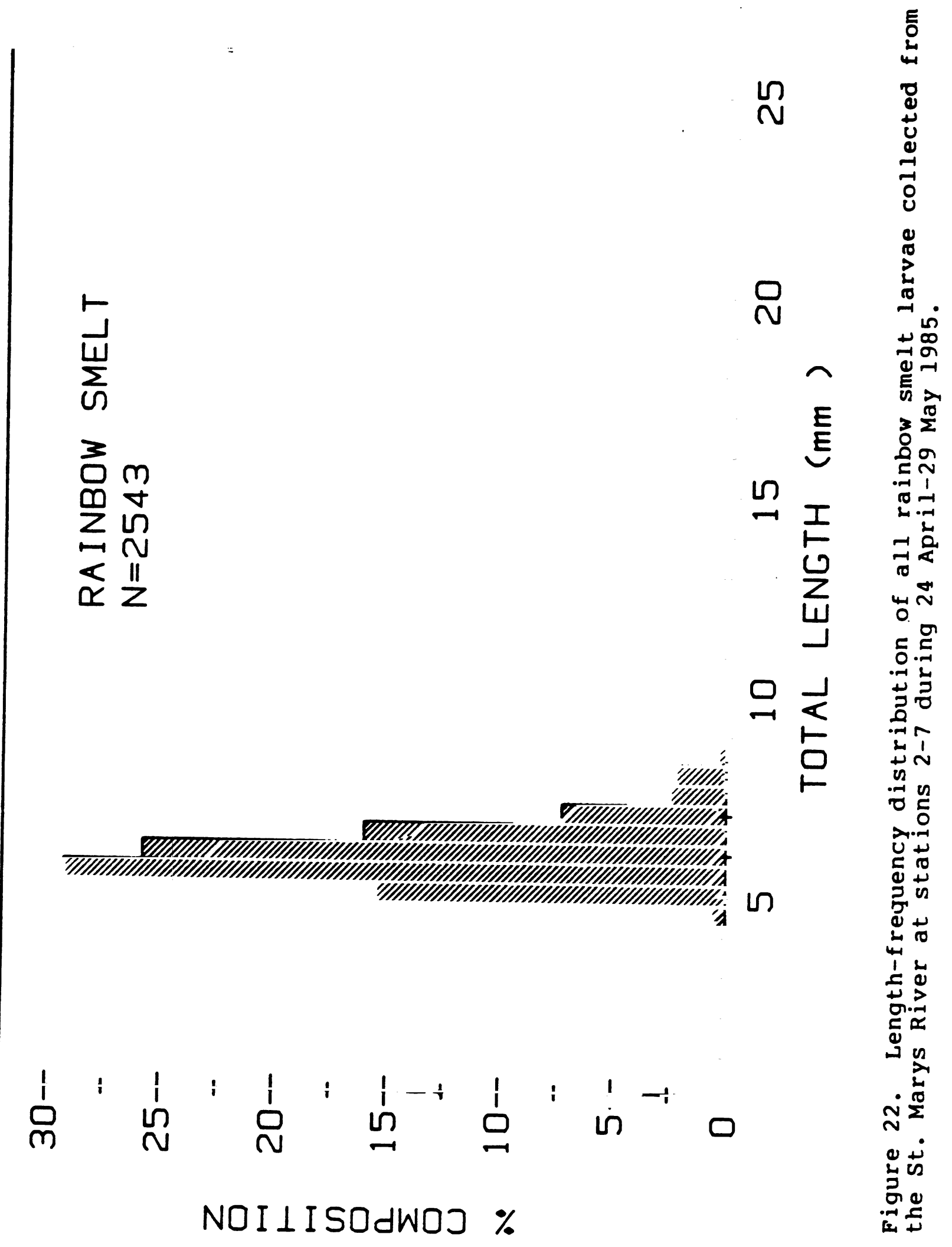


Mean sizes of yellow perch changed slightly over the 3 sampling wh. Larvae ranged from 5.5 to $10 \mathrm{~mm}$ with one mode at $7.5 \mathrm{~mm}$ (Fig. 23). This suggests continual hatching over this period. Sizes of larval yellow perch recorded by Liston et al. (1986) did not increase substantially until the beginning of June.

In summary, the St. Marys River, especially Lake Munuscong, is very productive for yellow perch larvae. Spawning must occur in late April to mid-May and larval fish densities peak in late May.

\section{Other Species}

Three emerald shiner (Notropis atherinoides) larvae from 20.5 to $22.5 \mathrm{~mm}$ were collected during the first week of sampling at stations 6 and 7 . Since we arbitrarily defined larvae as any fish $\leq 25.4 \mathrm{~mm}$, we sometimes, as in this case, collect $f$ ish which were hatched the year before. A similar case occurred for spottail shiner. The $f$ ish collected were also yearlings produced in 1984. They ranged in length from 19 to $25 \mathrm{~mm}$ and in density from 6 to $1,283 / 1,000 \mathrm{~m}^{3}$. Spottail shiners were present during all weeks of sampling except the first, and occurred at four (stations 4-7) of the seven stations sampled.

There were four deepwater sculpin (Myoxocephalus thompsoni) collected during the study; they ranged from 10.0 to $17.1 \mathrm{~mm}$. They were collected at the Edison Hydropower Canal (week 3 ), in the channel at stations 3 and 4 (week 4), and in the channel at station 7 (week 5 ). We concluded from these data that these larvae probably originated from Lake Superior, as they were exclusively collected in the channel where passive transport would be most probable. Also, deepwater sculpin are not known to spawn in connecting river systems, but are believed always to spawn in deep waters of the Great Lakes (Scott and Crossman 1973; Mansfield et al. 1983). Because of their large size, the larvae we collected had probably hatched some months before. Auer (1982) reported that newly hatched deepwater sculpin have not been described, but that larvae $8.2 \mathrm{~mm}$ (not newly hatched) have been described. Some of our larvae were twice that size.

\section{Fish Egas}

We collected $f$ ish eggs in many of the samples collected in the latter weeks of the study. None of these were lake herring or lake whitefish eggs, as they all were less than $2 \mathrm{~mm}$, the minimum size of the eggs of these species. Many had distinctive stalks, a unique characteristic of rainbow smelt eggs. Rainbow smelt eggs are about $1 \mathrm{~mm}$ in diameter, which is the size of the eggs we collected. 


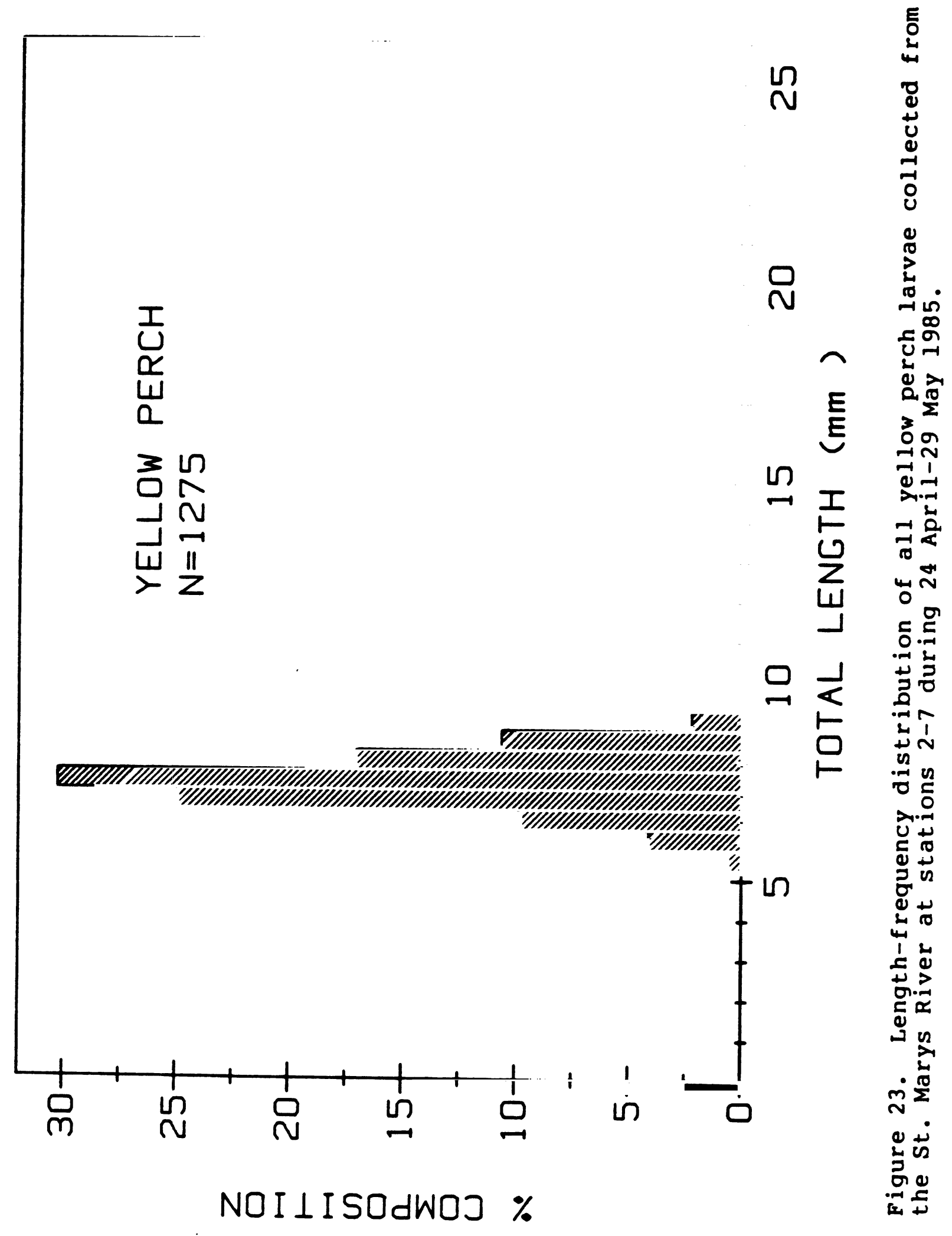




\section{Summer Drift and Biomass of Larval Fish}

Larval fish drift was measured during the winter (none caught) and summer, when large numbers of mostly rainbow smelt were collected. A detailed discussion of larval $f$ ish drift can be found in the section entitled: RESULTS - DRIFT: BENTHOS, FISH LARVAE, FISH EGGS, AND MACROPHYTES - Seasonal and Transect

Comparisons of Drift Densities - Fish Larvae. A brief discussion will ensue here. Rainbow smelt $(82 \%)$, burbot $(0.6 \%)$, lake herring $(0.1 \%)$, yellow perch and deepwater sculpin $(<0.1 \%)$, and damaged and unidentified larvae ( $18 \%$ ) made up the species collected during drift sampling. Rainbow smelt were mostly newly hatched, as larvae 4.5-6 mm made up over $90 \%$ of the catch (Fig. 24). Burbot larvae collected remained as small as those collected during our spring larval $f$ ish survey, about $4 \mathrm{~mm}$ (Fig. 24). However, a few larger burbot up to $6 \mathrm{~mm}$ were collected. Damaged larvae were also small, ranging from 3 to 6 $\mathrm{mm}$ (Fig. 24). We believe most of these larvae were damaged rainbow smelt. 

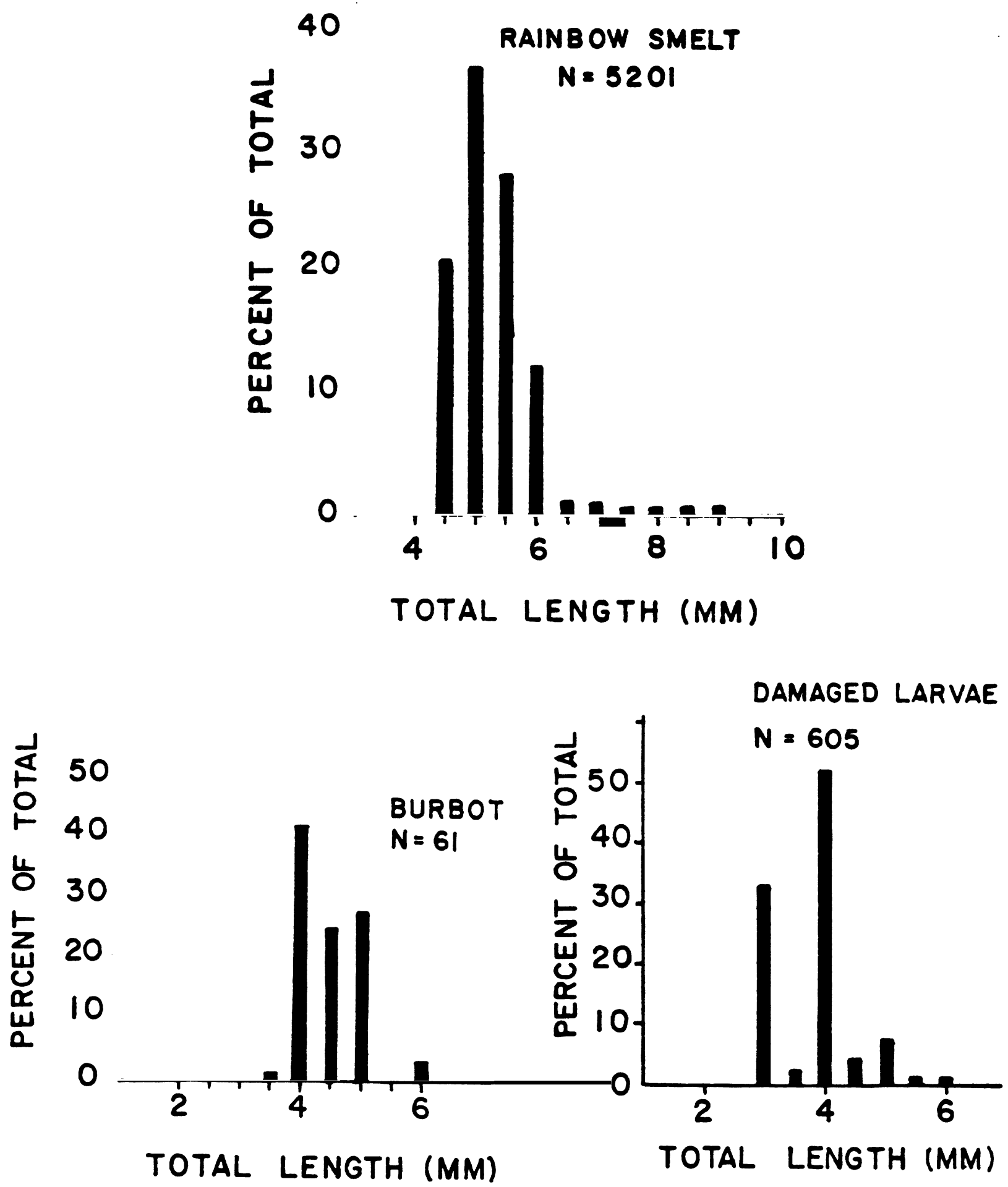

Figure 24. Length-frequency distribution of rainbow smelt, burbot, and damaged larvae collected from the St. Marys River during summer drift sampling, 1985. 


\section{DISCUSSION}

\section{MACROPHYTES}

Macrophyte data show a strong relationship between the maximum depth at which these plants occur and the degree of penetration of light through the water, which is in agreement with the findings of Liston et al. (1986). The stations in the lower part of the river, such as Lake Munuscong and Raber Bay, have greater water turbidity and, therefore, shallower outer macrophyte boundaries. Not only is the water more turbid at these places than at upstream sites such as Izaak Walton Bay and Lake Nicolet, but also more variable in turbidity, as shown by the greater width of the confidence intervals for mean light extinction coefficient (Fig. 7). This point is also apparent from casual observation during our studies in the river, for much more spatial and temporal variations in water clarity. were seen in the downstream portions than upstream. On one occasion, in the course of several hours while we sampled the plant transect at Raber Bay, a mass of turbid water gradually approached and engulfed the site. The increasing variability in water turbidity with distance downstream is probably related to greater contributions by tributary streams and rivers, which add great volumes of turbid water, particularly during periods of high runoff. There are very few major tributary streams emptying into the St. Marys River between Izaak Walton Bay and Lake Nicolet, but a number of important tributaries downstream.

Although water clarity is a determinant of the maximum depth at which aquatic macrophytes will grow, it is not the sole determinant of the location of the boundaries of plant beds. Sampling of plants along the $1-\mathrm{km}$ transects showed that substrate characteristics also affected plant distribution. Plants were abundant on clay substrates and much less so on sand or gravel bottoms. The large number of grab samples containing no plants in the east Lake Munuscong transect (Table 4) is due to the variable nature of the substrate in that area, which contained some large patches of sand.

Other, less obvious factors may also control the maximum depth of plant occurrence. For instance, in the course of searching for a site for the l-km transect in Lake Nicolet, we investigated a deep "trench" in the area 2-4 km south of Wasig Bay. Despite the clay substrate and relatively transparent waters at this location, plants were not found growing at depths as great as at the place where the transect was eventually located, some $6 \mathrm{~km}$ to the south. The macrophyte beds also tended to be more patchy at the former site than at the latter. The reason for this difference is not readily apparent; further investigations may be warranted. 
These observations suggest that any estimates of possible effects on submerged macrophyte beds in the St. Marys River resulting from natural or man-made perturbations must take into account not only possible changes in water turbidity, but also changes in the river bottom substrate. It would also be necessary to have more detailed data on the location, coverage, and depth of macrophyte beds along the length of the river prior to such perturbations to assess their effects.

\section{BENTHIC DRIFT}

\section{Comparison with Previous Benthic Studies on the St. Marys River}

Whole River Comparisons--

Direct comparison of whole river trend results of the present drift study with those of a survey of benthic density and diversity (Liston et al. 1983, 1986) are tenuous because of the time differential, locational differences within the river, and level of taxonomic detail. Nonetheless, some comparisons of whole river trends for percent composition of the benthos and drifting benthos are possible. The number of benthic taxa collected in Liston's benthos survey (162 taxa) was considerably greater than the 71 drifting benthic taxa. Much of this difference is due to generic identification of the Chironomidae in the benthos survey not afforded to the drift study. of 14 drifting benthic taxa not encountered in the benthic surveys, most notable were Mysis relicta, Pontoporeia hoyi, Chaoboridae, and Psycodidae. Poe et al. (1980) also collected mysids and chaoborids in drift samples but not in benthic samples in the St. Marys River. With great likelihood, $M$. relicta and $P$. hoy $i$ collected in the present study originate from Lake Superior and are only temporarily residents in the slower flowing, deeper portions of the river. Seagle et al. (1982) reported a preponderance of chaoborids in drift samples which were not present in the same proportion in benthic samples of the Illinois River. In this latter study, chaoborids were thought to originate from slow, backwater areas connected to the Illinois River, or from the river itself in areas of slow current. Chacboridae, being collected only at Frechette Point, possibly occurs only in the upper, deeper, slow flowing portions of the river. However, there remains the strong possibility that the major reason chaoborids occurred only in Frechette Point drift samples is that chaokorids migrated up into the water column in slower, upstream water, possibly even backwater portions of the river, and were drawn or flushed into the fast flowing water above Frechette Point by currents and by their own nocturnal activity. Farther downstream where current velocities are considerably slower than at Frechette Point they likely resettle. This being the case, chaoborids probably inhabit the slower, 
deeper portions of the entire river proper as well as connecting backwaters.

Psycodidae occurred only in a limited number of winter Frechette Point drift samples. Psycodids are known to inhabit quiet, very organically enriched waters (Hilsenhoff 1975). Absence of psycodids in benthic samples, coupled with a limited presence in drift samples, suggested the source of their occurrence may be external and not part of a sustained river supported population. A possible source may be an upstream sewage plant or factory input. This is suspected because concurrent with occurrence of psycodids, but not limited to samples in which psycodids occurred, was a clear, entangled, fibrous material. This material was the dominant component of all winter drift samples at Frechette Point and decreased substantially in a downstream direction.

The general lack of drift density differences across stations within each transect differed from benthic distribution patterns. Liston et al. (1986) found consistently greater benthic densities along the western, leeward side of the river than along the eastern, windward side. They attributed the difference to wave action scouring the bottom on the eastern side of the river. This trend was observed only at Frechette Point during the winter. None of the remaining transect station drift densities during either season exhibited this windward/leeward trend.

On a river-wide basis, Liston et al. (1986) reported only percent occurrence among samples collected. Agreement between percent occurrences for dominant taxa and between order of dominance within each survey was moderate at best. Percent occurrence for each taxon in benthic samples was greater than in drift samples (Table 44). While Chironomidae occurred in greatest frequency in both surveys, remaining orders of dominance differed considerably. Notable high percent occurrences in benthos samples but not in drift samples were recorded for Ceratopogonidae, Hexagenia, Ephemera, Caenis, Polycentropus, Polychaeta (Manayunkia speciosa), and Mystacides. Among present drift samples, notable high percent occurrences not observed among Liston's benthic samples included those for Hydra, Hydracarina, and Mysis relicta. Although percent occurrence differed between the two surveys, all major benthic survey taxa occurred in drift samples.

Comparisons at Frechette Point--

Based on Ponar grab samples from Frechette Point and Six Mile Point by Poe et al. (1980), estimated benthic density was $14,126 / \mathrm{m}^{2}$; of 75 benthic taxa collected, $25 \%$ was Chironomidae, 


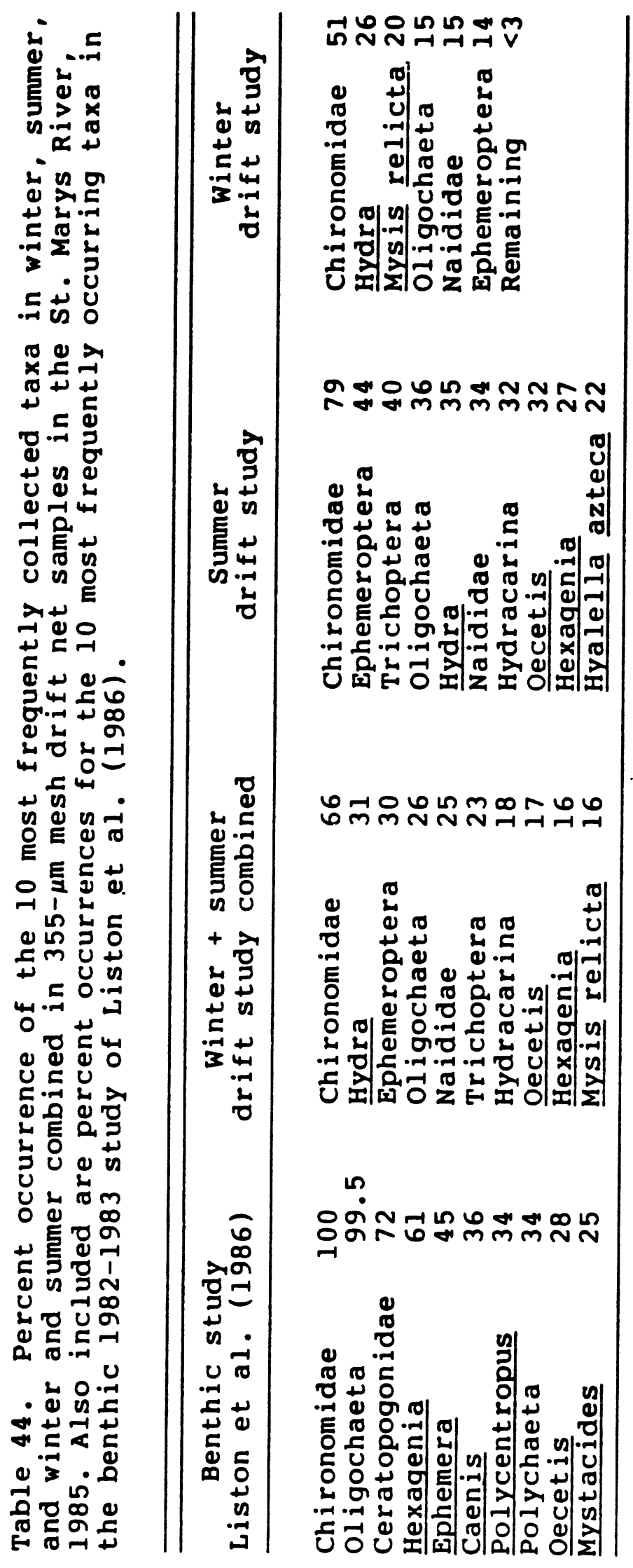


23\% Oligochaeta, 20\% Gastropoda, $11 \%$ Pelecypoda, $7 \%$ Polychaeta, $3 \%$ Amphipoda, $1 \%$ each for Ephemeroptera and Trichoptera, and $10 \%$ miscellaneous. While mollusks were seldom collected in drift samples, percent composition of the 64 drifting benthic taxa was fairly similar to that of the bottom population. Chironomidae made up 31\%, Naididae 9\%, Trichoptera $3 \%$, and Ephemeroptera $2 \%$ of the drifting benthos (Table 9). However, there remained a considerable difference between the benthos of the two studies, because in our drift study Hydra made up $49 \%$ and Chaoboridae $3 \%$ of the drift at Frechette Point.

Comparisons at Lake Nicolet--

Station II of Liston et al. (1986) corresponded to Lake Nicolet stations $1-5$ in the present drift study. In Liston's study, the Chironomidae dominated $(33-85 \%)$ benthic densities which ranged from $4,800 / \mathrm{m}^{2}$ to $34,700 / \mathrm{m}^{2}$, excluding the navigation channel $\left(294-1,750 / \mathrm{m}^{2}\right)$. Oligochaeta made up from $<1 \%$ to $64 \%$ of benthic density. Hyalella azteca made up $10 \%$ of benthic abundance. Although Trichoptera and Ephemeroptera were represented by several taxa, neither was numerically important, except Caenis which at maximum made up $18 \%$ of benthic density. Polychaeta was occasionally numerically important, making up to $17 \%$ of benthic abundance.

By comparison, in our drift study, Chironomidae made up $42 \%$ of average summer benthic drift at Lake Nicolet. The second- and third-most abundant drifting benthos, Ephemeroptera (37\%) and Trichoptera (9\%) (Table 9), as well as presence of M. relicta differed considerably from benthic composition in Liston's study. Nonetheless, dominant, frequently occurring genera common to the benthos of each study were often the most frequently collected taxa in our drift study.

Comparisons at Point aux Frenes--

Station VII of Liston et al. (1986) corresponded with the Point aux Frenes transect in the present drift study. Benthic densities ranged from $1,500 / \mathrm{m}^{2}$ to $29,900 / \mathrm{m}^{2}$ outside the navigation channel and $700 / \mathrm{m}^{2}$ to $2,300 / \mathrm{m}^{2}$ within the channel. Chironomidae was the dominant benthic taxon (22-85\% of benthic density), followed by Oligochaeta $(6-80 \%)$. Liston et al. (1986) noted that benthic diversity was lowest at Point aux Frenes (55 taxa). This corresponded with results from our drift study in which a study minimum of only 25 benthic taxa was collected which were made up mostly of Hydracarina, Chironomidae, Ephemeroptera, and Corixidae in order of decreasing numerical importance. This order differed considerably from the order found by Liston et al. (1986) in the benthos. 
Comparison with Previous Drift Studies on the St. Marys River

Direct comparison of station and transect benthic drift densities between the present study and those of Poe et al. (1980) and Poe and Edsall (1982) is limited to Frechette Point. The Lake Nicolet and West Neebish transects of Poe and Edsall (1982) are not comparable to the present Lake Nicolet transect, because the former was located considerably northward near Six Mile Point at the upper end of Lake Nicolet and the latter was located somewhat southward in the West Neebish Channel. The present Lake Nicolet transect was located in the main body of water in lower Lake Nicolet between Poe's transects. For comparisons between the two studies, Poe's winter benthic drift densities were averaged for each station (1-3) at Frechette Point by pooling across the 31 January 1982 through 9 March 1982 samples periods during which the river was under ice cover. Poe's drift density during ice-free conditions was represented by the 29 April 1982 to 1 May 1982 sample period for stations 1-3 and for the navigation channel (station 4; only daytime samples collected). Average transect drift density was estimated by pooling across all station and sample dates for ice-covered and ice-free conditions, respectively, in the study of Poe and Edsall (1982). In our study, comparison of benthic drift density with that of Poe et al. (1980) and Poe and Edsall (1982) was made by pooling in the same manner as above for stations $1-3$ and for the navigation channel (station 4; Day 1 and Day 2 averaged) at Frechette Point. Comparisons between the two studies was based on drift collection by Poe using 571- $\mu$ m-mesh nets and in our study, 355- $\mu \mathrm{m}-\mathrm{mesh}$ nets.

Poe's 1982 winter average benthic drift density for stations 1-3 ranged from $43 / 1,000 \mathrm{~m}^{3}$ to $63 / 1,000 \mathrm{~m}^{3}$, with a transect mean of $47 / 1,000 \mathrm{~m}^{3}$. In 1985, similarly pooled estimates for stations 1-3 ranged from $680 / 1,000 \mathrm{~m}^{3}$ to $1,216 / 1,000 \mathrm{~m}^{3}$, with a transect mean of $898 / 1,000 \mathrm{~m}^{3}$. In summer, Poe's station drift densities ranged from $42 / 1,000 \mathrm{~m}^{3}$ to $603 / 1,000 \mathrm{~m}^{3}$ and averaged $64 / 1,000 \mathrm{~m}^{3}$ in 1982. During our study in summer 1985, similar estimates ranged from $1,456 / 1,000 \mathrm{~m}^{3}$ to $1,884 / 1,000 \mathrm{~m}^{3}$ and averaged $1,659 /$ $1,000 \mathrm{~m}^{3}$. In Poe's 1982 navigation channel samples, daytime benthic drift density averaged $183 / 1,000 \mathrm{~m}^{3}$ and decreased from surface $\left(236 / 1,000 \mathrm{~m}^{3}\right)$ to mid-depth $\left(177 / 1,000 \mathrm{~m}^{3}\right)$ to bottom $\left(135 / 1,000 \mathrm{~m}^{3}\right)$. In our 1985 navigation channel samples, daytime benthic drift density averaged $1844 / 1,000 \mathrm{~m}^{3}$ and increased from surface $\left(721 / 1,000 \mathrm{~m}^{3}\right)$ to $\mathrm{mid-depth}\left(1,630 / 1,000 \mathrm{~m}^{3}\right)$ to bottom $\left(3,183 / 1,000 \mathrm{~m}^{3}\right)$. When the 1985 to 1982 ratios of pooled transect average (stations 1-3) densities were calculated, the 1985 winter, under-ice benthic drift densities we obtained were $1,911 \%$ greater than in 1982; under ice-free conditions in 1985, 
our estimates were $2,592 \%$ greater than 1982 estimates. The same ratio for ice-free navigation channel estimates indicated 1985 surface $(306 \%)$, mid-depth $(921 \%)$, bottom $(2,358 \%)$, when averaged over all three water depth strata $(1,008 \%)$, were considerably greater than the 1982 estimates. While some of these differences may be attributable to annual variation and slight variations in seasonal sampling times, the primary factor influencing these differences is the mesh size employed.

The reversal of depth of maximum drift density in the water column between the present study and that of Poe and Edsall (1982) is unexplainable. However, similar studies on the Mississippi River have been inconclusive as well. Matter and Hopwood (1980) found a drift density difference between depth strata which was related to specific taxa. In their study, ephemeropterans were most numerous in the upper nets at night while trichopterans were most abundant in lower nets regardless of the diel period. Seagle et al. (1982) observed a similar trend for ephemeropterans and Chaoborus which were most numerous near the surface and for hydropsychid trichopterans which were most numerous near the bottom in both the Mississippi River and Illinois River. In another study on the Mississippi River, Eckblad et al. (1983) noted depth of maximum drift density varied with season. During June, drift was greatest near the bottom and was comprised largely by Hydra. However, during July, drift, largely Corixidae, was maximal near the surface. At Point aux Frenes in the present study, corixids increased in drift density in the summer but were most numerous near bottom (Table 7). In fact, nearly all taxa including Ephemeroptera and Chaoboridae occurred in greatest densities in bottom nets. The taxa least limited by water column depth was $M$. Ielicta which being a good swimmer, active migrater, and emigrating form from Lake Superior, is not limited by the shallow depths of the river. While results from the present study strongly indicated most drifting benthos occurred near bottom, seasonal factors may alter the observed pattern.

\section{Some Factors Influencing Drift}

\section{Benthic Density and Production--}

Several studies have reported no direct relationship between drift and benthic population composition, standing crop, or production (Elliott 1967; Morris et al. 1968; Bishop and Hynes 1969; Waters 1972). Nonetheless, some studies have shown that drift was density dependent (Mulier 1954a; Waters 1961, 1962a; Pearson and Franklin 1968). Additionally, Waters (1972) speculated that drift may reflect excess production. Ghetti and Ravanetti (1984) reported a positive correlation between drift density and productivity, noting drift increased around emergence 
periods. Although Pearson (1970) was able to demonstrate a good correlation between drift and production for a caddisfly, none could be shown for a co-occurring mayfly.

The discussion offered by Bishop and Hynes (1969) regarding drift, carrying capacity, and production perhaps best relates the interaction of these concepts. Density-dependent drift is least evident in streams which are subject to spates and severe disruption. In examples of density-dependent drift, amphipods and multivoltine ephemeropterans dominated the benthos. As a consequence, drift was influenced by the density of the these invertebrates and their excess productivity. However, in streams experiencing spates or disruptions, carrying capacity is not attained. Subsequent drift results from behavioral activity and not from excess production. Regardless of these arguments, Bishop and Hynes (1969) concluded that the relationship between drift and production is "obscure."

In the St. Marys River, little is known empirically about its benthic carrying capacity. The only estimates of biomass and production are for $1-m$ and $3-m$ deep stations in lower Lake Nicolet and Lake George during 1981 (Liston et al. 1983). At the $1-\mathrm{m}$ station, average biomass was $6,387 \mathrm{mg} / \mathrm{m}^{2}$. Total annual production for common benthic taxa was $11,319 \mathrm{mg} / \mathrm{m}^{2} / \mathrm{yr}$. Production estimates in excess of $2,000 \mathrm{mg} / \mathrm{m}^{2} / \mathrm{yr}$ included those for Chironomidae and Isopoda. At the $3-\mathrm{m}$ station, average annual biomass in 1981 was $4,550 \mathrm{mg} / \mathrm{m}^{2}$. Total annual production was $10,704 \mathrm{mg} / \mathrm{m}^{2} / \mathrm{yr}$, with production estimates in excess of $2,000 \mathrm{mg} /$ $\mathrm{m}^{2} / \mathrm{yr}$ for Chironoidae and Amphipoda.

Biomass and production estimates from the St. Marys River Frovide some useful information but given the lack of clarity regarding the effect of biomass and production on drift, are of minimal use in explaining our data. Nonetheless, several of the more numerous and productive invertebrates did appear frequently in drift samples suggesting these factors should not be ignored. In addition to biomass and production, several other factors may exert a controlling influence on drift. Based on our understanding of these factors, they are interdependent to some degree and require simultaneous consideration.

Benthic forms which are most inclined to drift include in order of quantitative importance: Ephemeroptera, Simuliidae, Trichoptera, and Plecoptera (Waters 1972). The likelihood of each drifting is dependent upon life stage, current velocity, total discharge, temperature, light intensity (Elliott 1967; Bishop and Hynes 1969; Waters 1972), and in the present study, vessel traffic, and weather conditions. The latter two factors will be considered in a subsequent section. 


\section{Life Stage and Current Velocity--}

Aquatic insects in later, larger life stages are the most likely individuals to drift (Waters 1972). One mechanism influencing drift of these individuals is a need for increased foraging areas, thereby subjecting them to increased exposure to currents, jostling of one another causing one or more to become dislodged, or movement into the water column for emergence (Mundie 1956; Bishop and Hynes 1969; Waters 1972; Bailey 1981; Morgan and Waddel 1981). Any or all of these life stage factors will be species specific with subsequent drift composition not adequately reflecting either the pre- or post-benthic population structure. Moderate agreement between drift and benthic compositions must certainly reflect these life stage factors and account for a portion of the difference observed.

A most notable disagreement between our drift study and those of Poe et al. (1980) and Liston et al. (1986) was very frequent occurrence of Hydra and Mysis relicta in drift samples but not in benthos samples. However, Hydra did make up a large percentage of total benthic drift density in the high impact area at Frechette Point where Poe et al. (1980) sampled. Comparative occurrences of these two invertebrates suggest 1) even though mysids are not easily caught by Ponars, they are very likely not long-term residents of the river, but rather originate in Lake Superior and are either transported to Lake Huron, consumed by fish, or die of natural causes before passing out of the river, and 2) that Hydra occurred in substantive quantities upstream of Frechette Point, possibly on rocks and boulders, plants, and pilings and other man-made structures that were not or could not be sampled by usual benthic sampling devices. Greater drift densities of Hydra at Frechette Point relative to Lake Nicolet and Point aux Frenes suggested favorable, upstream current conditions enabling them to establish a large population which quite possibly preys heavily on zooplankton originating in Lake Superior.

Occurrence of large numbers of Hydra in the drift may be due to a force, such as current, causing dislodgement. Winter drift densities were lower than those in summer, and winter Hydra were larger sized $(3-5 \mathrm{~mm})$ than those in summer ( $<2 \mathrm{~mm}$, many $<1 \mathrm{~mm}$ ). Large summer densities of small Hydra strongly indicated reproductive activity and dispersal of young to new areas of attachment. We conclude that occurrence of Hydra in the drift in the present survey was induced primarily by current in winter but largely by reproductive activity in summer over and above some residual level attributable to current.

With respect to remaining drifting benthic taxa, current velocity appeared to influence drift density. In contrast to Hydra, remaining taxa are mobile and subject to contact with 
currents and subsequent entrance into the drift through their own movement. Consistently greater drift densities at Frechette Point relative to Lake Nicolet and Point aux Frenes were likely related to higher current velocities.

\section{Total Discharge--}

Little can be stated regarding the effect of total discharge on drift density in the St. Marys River as discharge is controlled at the locks and varied little over the course of the two time periods sampled. In smaller streams where most drift studies have been conducted, total discharge is often an overriding factor influencing increased drift due to flooding. While flooding was not a factor in our study, drift rates and intensities are related to discharge. Calculated drift intensities in our study are all well below maximal values reported by Waters (1972) who cited maximal drift intensities ranging from $2 \times 10^{\circ}$ to $3 \times 10^{\circ}$ in studies where all species were considered. Moreover, by substituting in the linear regression equation relating total discharge to invertebrate drift of Elliott (1970), we calculated a drift intensity of 27,681 . If a range of $\pm 50 \%$ about the 27,681 -value is calculated $(13,841$ $41,522), 37 \%$ of our values fall within this range, $21 \%$ were higher, and $42 \%$ were lower. To our knowledge, drift intensities have not been calculated for other large rivers, and given agreement between our values and those of Elliott (1970), even though his were based on streams, we feel assumptions inherent in our calculations and results obtained were reasonable. However, we acknowledge the differences between expected and calculated discharges and offer that more frequent measures would enhance future estimates of drift rates and drift intensities. At best, our estimates may be thought of as a first approximation.

When examining benthic drift density, drift rate, and drift intensity seasonal ratio differences among transects, the degree of seasonal change was greatest at Lake Nicolet when compared with Frechette Point (Tables 10-11). In addition, the degree of difference between these two transects decreased from winter to summer (Table 45). Common to Frechette Foint and Lake Nicolet transects is a similar seasonal reproductive and behavioral activity of the benthic organisms. Differing between the transects is the physical structure of the river basin at each transect location which in turn affects current velocity. We speculate that while reproductive and behavioral activity of the benthos at Frechette Point increased during summer when compared with winter, the high current velocities observed during both seasons dampen seasonal differences to a greater degree at Frechette Point than at Lake Nicolet. At Lake Nicolet where seasonal ratio differences were considerably greater than at Frechette Point, we suspect that reproductive and behavioral 
Table 45. The ratio between pairs of transects of benthic and larval $\mathrm{fish}$ densities, rates, and intensities in the $S t$. Marys River, 1985. FP = Frechette Point, $L N=$ Lake Nicolet, PAF = Point aux Frenes. See METHODS and Waters (1972) for definition of drift intensity.

\begin{tabular}{|c|c|c|c|c|c|c|}
\hline \multirow[b]{2}{*}{$\begin{array}{c}\text { Transect } \\
\text { ratio }\end{array}$} & \multicolumn{3}{|c|}{ Winter } & \multicolumn{3}{|c|}{ Summer } \\
\hline & $\begin{array}{l}\text { Drift } \\
\text { density }\end{array}$ & $\begin{array}{r}\text { Drift } \\
\text { rate }\end{array}$ & $\begin{array}{c}\text { Drift } \\
\text { intensity }\end{array}$ & $\begin{array}{l}\text { Drift } \\
\text { density }\end{array}$ & $\begin{array}{r}\text { Drift } \\
\text { rate }\end{array}$ & $\begin{array}{c}\text { Drift } \\
\text { intensity }\end{array}$ \\
\hline $\begin{array}{c}\text { Benthos } \\
\text { FP/LN } \\
\text { FP/PAF } \\
\text { IN/PAF }\end{array}$ & $\begin{array}{l}9.84 \\
2.30 \\
2.33\end{array}$ & $\begin{array}{r}17.3 \\
-\end{array}$ & $\begin{array}{r}26.5 \\
- \\
-\end{array}$ & $\begin{array}{l}2.71 \\
3.23 \\
1.19\end{array}$ & $\begin{array}{l}5.22 \\
6.33 \\
1.21\end{array}$ & $\begin{array}{l}5.50 \\
11.6 \\
2.12\end{array}$ \\
\hline $\begin{array}{l}\text { Fish } \\
\text { LN/FP } \\
\text { LN/PAF } \\
\text { PAF/FP }\end{array}$ & $\begin{array}{l}- \\
-\end{array}$ & $\begin{array}{l}- \\
-\end{array}$ & $\begin{array}{l}- \\
-\end{array}$ & $\begin{array}{l}11.3 \\
72.7 \\
0.16\end{array}$ & $\begin{array}{l}94.1 \\
38.2 \\
2.46\end{array}$ & $\begin{array}{l}89.3 \\
66.8 \\
1.34\end{array}$ \\
\hline
\end{tabular}


activity may be a more important factor influencing seasonal ratio differences than the effect of current velocities which were slow relative to those at Frechette Point. To express these relationships numerically, we found that at Frechette Point, the constant, eroding effect of higher current velocities on the benthos allowed a three-fold increase due to seasonal

reproductive and behavioral activity. At Lake Nicolet where the eroding effect of currents is less than at Frechette Point, there was a ten-fold increase in the drift ratios. We speculate most of the increase is due to activity of the organisms. However, some seasonal difference may be related to the type of organisms in the drift which in turn are influenced by the physical nature of the basins they encounter while drifting. A good comparison of this effect is between Hydra and Mysis relicta. While both animals drift through Frechette Point, the former was caught in our drift nets with much greater frequency at Frechette Point than the latter. However, at Lake Nicolet, mysids were retained in greater relative frequency than were Hydra. This difference is related to animal mobility and the influence of these two segments of the river on the animals. Neither animal is expected to be able to resettle in the high current velocity at Frechette Point. However, upon reaching the slower currents in Lake Nicolet, Hydra, being sessile animals, resettle slowly, and mysids, being highly mobile animals, resettle quickly. Regardless of time of day, but most particularly at night, mysids, which are active migraters, reenter the drift but Hydra do not unless disturbed or unless they are in a reproductive phase of their life cycle. Consequently, while both animals pass through Frechette Point and presumably accumulate in Lake Nicolet, mysids were more evident in drift nets than were Hydra. The effect of mysids accumulating in Lake Nicolet would be expected to be more evident in drift samples due to their active migratory nature than for Hydra due to slower currents. The same effect may occur at Point aux Frenes but evidently to a lesser degree based on our data. Accumulation of mysids in lake Munuscong may be less intense owing to increased predation effects due to increased time spent in the river, siltation, temperature changes, or natural mortality. Additionally, the large, slow-moving water mass in Lake Nicolet apparently provides an excellent nursery for $f$ ish which is not present at Frechette Point. We suspect the physical difference among transects contributes to benthic and larval fish drift differences among transects as they interact with the behavioral characteristics of a given taxon.

\section{Water Temperature--}

The effect of water temperature on drift within respective seasons was minimal, because water temperature differences among stations within transects and among transects were negligible. 
However, water temperature acting in concert with other factors considered in this section likely had a positive effect on seasonal drift differences. Water temperature directly affects development rates and physical conditions in the river, such as ice conditions. Waters (1962b) and Pearson and Franklin (1968) observed increased drift with increased water temperature. Muller (1954b) noted a decrease in drift at lower temperatures, and Waters $(1962 a, 1966,1981)$ found low drift in winter. Lower benthic densities in winter, decreased water temperature, and slower developmental rates in winter with their converse in summer, coupled with input of young, newly recruited individuals into the system, likely accounted for a substantial portion of seasonal differences observed in the present study.

Results of studies evaluating the effect of anchor-ice on drift vary. Anchor ice increased the amount of drift in the study of Bishop and Hynes (1969). Logan (1963) concluded that surface ice cover had no effect on benthic density except near the edges of the stream. With spring break-up, floating surface ice did not increase drift (Logan 1963). This conclusion agreed with that drawn by Poe and Edsall (1982) that floe ice did not increase drift density. Nevertheless, we postulate that there remains a possibility that ship passage under conditions of ice break-up may increase drift when there is considerable anchor ice. This effect will most probably be more intense in slower flowing portions of the river where thicker ice develops and possibly, movement of nearshore ice blocks to offshore positions takes longer than in narrow portions of the river with higher current velocities. The surging of water and ice chunks in shallow water due to ship passage may be analogous to surging of ice blocks along the shoreline of Lake Michigan during ice breakup (Seibel et al. 1975). If so, there exists a strong possibility that nearshore benthic drift, where ice blocks might be expected to interact most with the bottom, will be increased above that of natural ice break-up. 'However, since wind exerted a dominant impact on drift during summer, the effect of wind on movement of ice blocks with subsequent nearshore scour may be a far more important force influencing additional drift than ship passage. Additionally, since shipping normally begins with ice break-up, neither the effect of shipping nor wind seem to have been detrimental to benthic populations. But in terms of factors potentially affecting drift, the scour of disturbed ice blocks on the bottom, regardless of the nature of the disturbance, is a factor which may increase drift of benthos.

\section{Light Intensity--}

The fact that benthic drift density is strongly dependent on light intensity has been reported by many investigators (Elliott 1967; Bishop and Hynes 1969; Waters 1972). Present results 
agreed well with the diel nature of drift activity, i.e., minimal drift during the day and maximal drift nocturnally. Elliott (1967) reported greatest drift just after sunset; the time when night samples were collected in the present study. While greatest diel drift density is species specific (Elliott 1970), barring catastrophic drift due to flooding or pollution, most drift results from behavioral activities (Bishop and Hynes 1969; Waters 1972). This brings the discussion full-circle to life stage, developmental factors. Increased drift at night is highly related to increased activity of invertebrates and to a lesser extent upon the carrying capacity of a particular stretch of river (waters 1972).

The Fate of Drifting Benthos and the Effect of Weather and Vessel Traffic on Benthos

Fate of Drifting Benthos--

There remains the question of the fate of drifting benthos. As with mysids, drifting benthos inherent to the river may drift out of the river, be consumed by a predator, die of natural causes, or resettle downstream. Natural factors influencing downstream drift distance include current velocity, length of nocturnal period, activity of the individual [size, shape, density, center of gravity, swimming ability (McLay 1970)], water temperature, substrate type, unoccupied downstream areas, and availability of slow water areas (Bishop and Hynes 1969; McLay 1970; Elliott 1971; Waters 1972).

Poe et al. (1980) speculated that macroinvertebrates settle out of the water column quickly relative to the more buoyant and passive macrophytic material. Bishop and Hynes (1969) noted that drift distance of invertebrates is not very long due to thigmotactic and rheotactic responses. Waters (1972) observed that aquatic insects characteristic of swift streams reattach rapidly when released into the water column and concluded it seemed likely that they do not swim freely or drift for long distances.

Mclay (1970) observed a range of downstream drift distances from $0.5 \mathrm{~m}$ to $19.3 \mathrm{~m}(\overline{\mathrm{X}}=11 \mathrm{~m})$. Elliott $(1970)$ found the rate of return of drifting benthos to substrates was a decreasing exponential function which decreased with increased distance from the point of origin of an individual into the drift. Average drift distance was $20 \mathrm{~m}$. Additionally, Elliott (1970) noted that Chironomidae, the most numerous drifting benthic form in the present study, resettled no more quickly than dead invertebrates. Nonetheless, $99 \%$ of them had resettled within $15 \mathrm{~m}$ in a current of $10 \mathrm{~cm} / \mathrm{s}$ and within $91 \mathrm{~m}$ in a current of $60 \mathrm{~cm} / \mathrm{s}$ (Elliott 1970). Although application of Elliott's findings to the 
St. Marys River which is a much larger and deeper river than the stream studied by Elliott $(16-40 \mathrm{~cm}$ deep, modal depth

approximately $20 \mathrm{~cm}$ ) may be erroneous, no other empirical methods for estimating drift distance exist. As a consequence, we have calculated a minimal resettling distance for Chironomidae in the St. Marys River using Elliott's equation values and assumptions. Due to the differences in river depths, we have also calculated an "adjusted drift distance."

At Frechette Point, average mid-depth navigation channel current velocity was $51 \mathrm{~cm} / \mathrm{s}$, which when substituted into Elliott's equation indicates $99 \%$ resettlement of drifting chironomids within $78 \mathrm{~m}$ in a stream of modal depth $20 \mathrm{~cm}$. Based on these measures, the resettling rate for the average benthos located at mid-depth $(10 \mathrm{~cm})$ in Elliott's stream would be 0.13 $\mathrm{cm} / \mathrm{m}(=10 \mathrm{~cm} / 78 \mathrm{~m})$. This assumes a linear rate of descent through a column of water travelling at a constant current velocity and direction. This resettling rate is used to calculate the "adjusted drift distance" for the average chironomid located at mid-depth in the navigation channel at Frechette Point $(4.5 \mathrm{~m})$. For chironomids located at this depth in the navigation channel at Frechette Point, $99 \%$ resettlement would be expected to occur within $3,462 \mathrm{~m}(=450 \mathrm{~cm} / 0.13 \mathrm{~cm} / \mathrm{m})$. The same organism located at mid-depth at the shallowest depth sampled (station $1=1 \mathrm{~m}$ ) would have a 998 resettlement within $192 \mathrm{~m}$ based on an average current velocity of $26 \mathrm{~cm} / \mathrm{s}$ and a resettlement rate of $0.26 \mathrm{~cm} / \mathrm{m}$. In a similar fashion, at lake Nicolet the "adjusted drift distance" at mid-depth in the upbound navigation channel is $1,440 \mathrm{~m}$, while that of the downbound channel is $1,710 \mathrm{~m}$. At Point aux Frenes, the "adjusted drift distance" at mid-depth in the navigation channel is $1,350 \mathrm{~m}$. At the shallowest stations sampled nearest these three channel locations, the "adjusted drift distances" are $35 \mathrm{~m}, 75 \mathrm{~m}$, and 60 $m$, respectively. From these calculations, we project 99\% resettlement of the main component of mid-depth, drifting Chironomidae within $60 \mathrm{~m}$ in the nearshore to approximately 3,500 $m$ in the navigation channel. Occurrence of faster or slower current velocities downstream will enhance or impede drift distance. However, based on these estimates, drift originating at mid-depth or bottom at any transect would likely resettle downstream prior to attaining the "adjusted drift distance" due to occurrence of slower water below each transect.

The exception to this may be those individuals occurring at the surface of the navigation channel. Expected drift distances for these individuals are $6,000 \mathrm{~m}$ at Frechette Point, 3,103 $\mathrm{m}$ in the downbound channel at Lake Nicolet, 2,727 $\mathrm{m}$ in the upbound channel at Lake Nicolet, and 2,647 $\mathrm{m}$ at Poirt aux Frenes. In these cases, as with those above, drift distances are anticipated to be shorter than calculated due to current variations. 
The more passive portions of the drift, such as macrophytic material, are expected to resettle in slower, downstream portions of the river. Although Poe et al. (1980) and Poe and Edsall (1982) speculated this material may be removed from the system upon being disturbed, we suspect it may be transported downstream to resettle in slow water areas (particularly Lake Nicolet and Lake Munuscong), and thereby, reassume its role in the river's production ecology cycle. While some may be lost from the lower reaches of the river, we suspect this is insignificant relative to the whole river and would not anticipate this altering benthic production negatively.

Once suspended in the water column organisms become easier prey for fish. The degree to which drifting benthos are utilized as a food by $f$ ish varies with the size and species of $f$ ish (Waters 1972). Selective feeding on drifting benthos occurs, particularly among young salmonids. However, this changes considerably as these $f$ ish get larger (Waters 1972). Waters (1972) concluded in general that fish are "opportunists" which often, but not always, consume drifting benthos.

Our speculation regarding the fate of drifting benthos in the St. Marys River is that a great proportion of the macroinvertebrates resettle within a short distance and that a small fraction is consumed or destroyed by drifting activity. Mysids may drift through the entire river system, but few other benthos would be expected to do the same. The majority of drifting Hydra probably perish or fare poorly when drifting from the northern portions of the river into Lake Nicolet unless by happenstance they resettle in suitable areas. Most other drifting benthos are capable of movement and reenter the drift to find suitable habitat.

\section{Effects of Weather and Vessel Traffic on Drift--}

Studies on the effect of ship passage on benthic drift are few and results are contradictory and seem to be dependent upon circumstances particular to each study. Herricks (1981) observed increased benthic drift following barge passage during low flow but not during high flow periods in the Mississippi River. Seagle and Zumwalt (1981) observed no increases in drift density due to "tow" passage in the Mississippi River, but attributed this to the confounding effect of high flow. Eckblad (1981) also demonstrated no significant increase in drift due to "tow" passage in the Mississippi River. However, northbound (upbound) "tows" in the Mississippi River increased drift at a 3-m sampling depth (Eckblad 1981). In addition, Eckblad (1981) noted that 9\% of the benthos attached to rock surfaces were dislodged with increased current velocity. However, Seagle and Zumwalt (1981) observed no such trend. 
The inability of the two ship passage studies in the present study to demonstrate detectable increases in the density of drifting benthos or $f$ ish larvae was probably related to the overriding effect of windy weather conditions. Based on visual observations, greatest impact on drifting benthic densities are expected to occur during upbound passage of vessels. We suspect this is due to the vessel plowing through the river and deflecting large volumes of oppositely flowing water slightly upstream and laterally, whereby it rushes downstream at a greater velocity than the ambient current velocity of the river. This process is a very dynamic one that eventually evens out the disturbance to reestablish the "normal" flow of the river at some equilibrium level. Downstream vessel passage apparently does not present such an extreme displacement of water at least in part because the ship and the water it is displacing are traversing the same direction. In fact, we observed a decrease in current velocity with downstream passage but an increase during upbound passage. This same trend was also observed by Eckblad (1981) in the upper Mississippi River. In either case, but especially for upbound ships, passage is expected to have a greater effect on increasing benthic drift depending on the speed and the size of the ship (Bhowmik et al. 1981). Under the windy conditions which we measured the effect of ship passage, no increase in drift density was observed. In a trial run of this experiment in calm weather during May 1985 at Frechette Point, we observed considerable disturbance of the bottom with increased turbidity during passage of an upbound vessel. Based on these observations, we conclude that there remains yet an unproven but highly probable increase in drift components with passage of an upbound vessel during calm weather conditions. However, during windy weather, the added effect of ship passage was undetectable and did not substantially alter an already disturbed system.

Drift induced by windy weather conditions and by ship passage during ice-free, calm weather conditions represents examples of quasi-catastrophic drift. It is difficult to ascertain the ultimate effect on the benthos of either and that effect very likely depends upon factors unique to each portion of the affected river bottom. However, we feel that windy-weather conditions have a greater overall, river-wide effect on drift than individual, though frequent, ice-free ship passages. In the most clear case of an effect due to weather, benthic drift density at Frechette Point, which should have been greatest nocturnally, was highest during the day. We feel this difference was due to windy conditions during the day and calm conditions during the night. This is a remarkable difference, because it suggests that wind intensity and direction is capable of exerting a greater controlling influence on drift than is light intensity. In addition to drift density differences, current velocities were greater during windy conditions than during calm conditions. This concurred with the conclusions drawn by the Great Lakes 
Hydraulics and Hydrology Branch of the U.S. Army Corps of Engineers which concluded that wind speed and direction influenced surface and probably subsurface flow of the St. Marys River (U.S. Army Corps of Engineers 1984). Similar results were observed during the larval fish surveys.

Wind-induced drift is expected to be river-wide, aside from sheltered areas. By contrast, ship-induced drift is pulsed by frequency of ship passage. In either case, induced drifting organisms will resettle, but we suspect they will do so more rapidly when induced by ship passage than by wind. More rapid resettlement of ship-induced drift is expected because the disturbance generated by a ship passage is short-lived and that caused by weather events may last for days or possibly weeks. In our 2-wk sample period in early June it was windy during daylight hours for all but 2 days.

If during calm conditions there are pulses of ship-induced drift, there remains the possibility that these "clouds" of drift may provide an unexpected food source for fish predators. In this fashion, ship-induced drift may add to the mortality of benthos populations in the river. However, we hypothesize that the effect of weather during ice-free conditions far exceeds normal ship passage effects on drift. Given the observed ability of river benthic and larval fish populations to maintain adequate productivity, we expect no adverse effect on these populations due to normal ship passage operations during ice-free conditions. Consequently, any loss of benthos attributable to normal shipping activity during the base shipping season of 1 April to 15 December is in all likelihood easily sustainable by the benthic population indigenous to the river.

Addressing the potential effect of ship passage on benthic populations in the river during winter, ice-cover conditions is much more difficult to answer. The primary reason is the lack of data. Poe et al. (1980) and Poe and Edsall (1982) demonstrated with their ship passage, under-ice drift data (the only data available) that there was a considerable increase in benthic drift density. This conclusion can not be overlooked and should be impetus for further investigation and analysis. However, reanalysis of the Poe et al. (1980) benthic drift data suggests vessel passage under conditions of ice-cover may result in a pulsed increase in benthic drift density (15-16 February 1979), while at other times (13-14, 17-18 March 1979) displaying little apparent effect. These variations leave open the solution to the effect of under-ice shipping effects and lead us to further speculation.

Based upon our knowledge of drift, we would not expect shipinduced drifting benthos to drift greater distances before resettling than during summer, thereby not causing any increased 
detrimental effect on existing populations. Most $f$ ish predators during winter are expected to be larger than during summer, probably located in deeper water, more lethargic, seldom feeding, and not relying heavily on drifting benthos as a food source. This being the case, predation may not be severe on a sudden pulse of drift moving downstream. However, we are concerned as were Poe et al. (1980) and Poe and Edsall (1982) about any additional loss to benthic populations at a time when numbers and productivity are minimal and about alteration or loss of preferred habitat due to physical disturbance of the river bottom. Benthic invertebrates which may be negatively affected include those capturing food by constructing nets or having a filtering apparatus, e.g., Hydropsychidae, some Chironomidae, Simuliidae, and Pelecypoda. Ephemeroptera would be negatively affected by increased siltation or sediment disturbances through fouling of gills and possibly loss of preferred habitat. Taxa which graze on algal growth on rocks or collect detrital material, e.g., many Trichoptera, Gastropoda, and many Chironomidae, may find those resources altered by physical disturbance of the river bottom. Alteration of habitat and particularly food resources during conditions of ice cover are important, because in winter the river is a much more static, fixed system than after ice break-up. When ice breaks up, benthic food resources increase, e.g., there is increased production of aquatic macrophytes and algae, and increased input of allochthonous material due to spring melt and rains, making any alterations less restrictive.

Whether the benthos can survive continued and frequent under-ice disturbances has yet to be demonstrated. Given that there was shipping under conditions of ice cover from 1977 through 1979 (personal communication; Don Williams, U.S. Army Corps of Engineers, Detroit District) and the benthos of the river apparently has been capable of maintaining reasonable densities and productivity rates in the areas and seasons studied, this suggests the effect of shipping has been minimal. However, the variable results observed by poe et al. (1980), and the concerns expressed herein remain to be addressed empirically. The data of Poe et al. (1980) do not seem adequate to answer whether the apparent ship-induced pulse of drift evident in their data would continue to be evident with additional ship passages or would level off at some equilibrium level. Regardless of which occurs, the impact of either on the benthos in high impact areas and possibly in low impact areas remains to be determined. Our speculation that previous shipping did not appear to detrimentally affect the benthos during ensuing years is not sufficient to forecast the future. The frequency of ship passage may vary from year to year and the fate and importance of induced drift losses remain unknown. This is particularly important to the cohort surviving from the previous reproductive period. Typically, in mid-winter and early spring this cohort will be at 
a numerical minimum. Finally, reproductive success of the benthos varies from year to year based on many factors but possibly foremost may be weather for taxa which have flying adults, e.g., Ephemeroptera, Trichoptera, and Diptera. Poor weather conditions at critical times, coupled with any potential additional loss attributable to ship passage, may be important to survivability of some taxa.

\section{ZOOPLANKTON}

\section{Zooplankton Abundance and Community Structure}

The St. Marys River was characterized by low zooplankton densities, both in February (winter) and early June (spring), averaging $313.2 / \mathrm{m}^{3}$ and $93.7 / \mathrm{m}^{3}$ respectively for No. 2-mesh net collections. For No. 10-mesh net collections, these averages were $636.2 / \mathrm{m}^{3}$ and $421.3 / \mathrm{m}^{3}$, respectively. In contrast, Selgeby (1975) reported that, in the outflow from Lake Superior, February zooplankton densities averaged approximately $2,000 / \mathrm{m}^{3}$ while early June densities averaged approximately $1,000 / \mathrm{m}^{3}$; his collections were made using a No. 10-mesh net.

Low zooplankton densities in our study appear to be due to several factors. First, most collections were made with a relatively coarse $(355-\mu \mathrm{m})$ No. 2-mesh net which undersampled all but the largest zooplankton. Evans and Sell (1985) determined that a No. 2-mesh net probably provides representative abundance estimates only for the largest zooplankton, i.e., Limnocalanus macrurus copepodites and possibly Daphnia. Immature Cyclops and Diaptomus copepodite abundances are severely underestimated by a No. 2-mesh net by at least one order of magnitude. Since zooplankton community structure in the St. Marys River varied seasonally and spatially, such underestimates were not constant over the study. For example, the St. Marys River zooplankton community was dominated by larger zooplankton in winter than spring (this study; Selgeby 1975). Furthermore, during June, zooplankton community structure varied along the length of the river with smaller-bodied zooplankton dominating at point aux Frenes while larger-bodied zooplankton were dominants at upper river transects at Frechette Point and Lake Nicolet.

A second factor which may have affected zooplankton abundance was the sampling method. Plankton nets were suspended in the river and zooplankton were collected as water flowed through the net. Current velocities were low, averaging $18.1 \mathrm{~cm}$; $s$ in February and $20.8 \mathrm{~cm} / \mathrm{s}$ in June. In contrast, towing speeds commonly used in zooplankton studies average 50 to $100 \mathrm{~cm} / \mathrm{s}$. These high towing speeds are necessary to minimize net avoidance, especially by the larger zooplankton. In this study, it is highly probable that a substantial fraction of the zooplankton 
community was able to avoid capture by the suspended nets, especially in areas where flow velocities were low. This may account for the fact that zooplankton generally tended to occur in highest densities in the channels, especially in surface waters, where current velocities were highest. This may also account for the greater densities of zooplankton in day versus night collections made in June at Frechette Point and Lake Nicolet.

There are two possible implications to sampling biases in zooplankton population estimates. First, seston (zooplankton and detritus) concentrations may have been underestimated; similarly, the relative contribution of detritus to total seston may have been overestimated. These errors probably varied with temporal and spatial changes in zooplankton community structure and current velocity. Second, if net avoidance was a significant factor affecting zooplankton drift abundances, it may also have been a significant factor affecting drift abundance estimates of other organisms. For example, fish larvae abundances probably were underestimated. The mean size of larvae collected in drift samples averaged only $11.9 \mu \mathrm{g}$. Benthic abundances may also have been underestimated, especially the highly motile Mysis relicta.

One intriguing aspect of the zooplankton study was the decline in zooplankton abundances at Point aux Frenes during winter and spring, and the decrease in mean animal size in June. The decline in abundance was most pronounced in June. Since current velocities at Point aux Frenes were higher than at Lake Nicolet, flow velocity through the plankton net does not appear to be an important factor. Possible important factors affecting the loss of zooplankton (especially larger-bodied animals) are mechanical damage in the turbulent river waters and predation by size-selective planktivorous fish.

Although most of the zooplankton community probably originated in Lake Superior (especially in winter), there were two other sources of animals. Small rivers and streams probably contributed to the St. Marys River zooplankton community. This was most evident at Frechette Point where a Daphnia morph, $D$. minnehaha was collected in low numbers in June at stations 2 and 3. A second probable source of zooplankton was the littoral and epibenthic regions of the St. Marys River, especially in summer. The epibenthic and littoral community appeared to be especially well developed at point aux Frenes in summer where there were large day-night, depth strata, and location (channel versus shallow) differences in mean animal size.

It is not clear whether or not an extension of the winter navigation season would have any effect on the zooplankton community. Although zooplankton abundances varied significantly along the course of the river, across the river, with depth, and 
with current velocity, the study was not designed to provide information on the causal factors. Thus it would be premature to speculate on what effects a potential increase in current velocity and resuspension (associated with an extended winter navigation season) may have on the zooplankton community along the course of the river.

\section{Biomass}

The biomass studies showed the variable nature of the river load. During winter, when the river was ice-covered, zooplankton was the major component of the river load (an average of $63.2 \%$ on a dry-weight basis). The second-most abundant component was detritus. The mean $8 A F W(a s h-f r e e d r y$ weight):seston was low $(4.8 \%)$, approximating the mean measured value for benthos, mysids, and fish. This suggests that the detrital component of seston was comprised largely of organic matter, e.g., plant fragments. In contrast, during the June study, zooplankton was a smaller component of river load (9.4\%) with detritus predominating $(86.9 \%)$. This occurred primarily due to a 5-fold increase in detritus biomass. The mean \&AFW:seston also increased to $25.6 \%$ suggesting that inorganic matter (various mineral particles) and refractile organic matter increased in concentration.

The June increase in detritus concentration and \&AFW:seston could have been due to two factors. First, as flow velocities of small rivers and streams increased with spring snowmelt, increased amounts of terrigenous material may have entered the St. Marys River. Second, as the river lost its protective ice cover, heavier sedimentary particles (with a higher \%AFW:sediment value) were more readily resuspended from the river floor as wind-driven waves activity intensified. Such wave activity could explain why detritus accounted for a larger percentage of the June river load at Point aux Frenes (98.7\%) than Lake Nicolet $(40.6 \%)$, although there were only small differences in current speed $(17.1 \mathrm{~cm} / \mathrm{s}$ versus $13.9 \mathrm{~cm} / \mathrm{s})$ between the two locations. Conditions generally were windy at the Point aux Frenes site but were calm at Lake Nicolet. Similarly, current velocities were higher at Lake Nicolet during winter $(18.9 \mathrm{~cm} / \mathrm{s})$ than spring $(13.9 \mathrm{~cm} / \mathrm{s})$, and detritus accounted for a smaller percentage of the total river load (27.0\% versus $40.6 \%)$. The mean $8 A F W:$ seston was lower in winter $(3.8 \%)$ at this site under ice cover than in spring $(24.6 \%)$ when ice cover had been lost. Current velocities apparently increased under windy conditions. For example, at Point aux Frenes, June current velocities averaged $17.1 \mathrm{~cm} / \mathrm{s}$ versus $4.0 \mathrm{~cm} / \mathrm{s}$ in winter. The June study was conducted during windy conditions. In contrast, differences in winter-spring current velocities were less at Frechette Point and Lake Nicolet; 
at these sites, June studies were conducted during calm or calm to windy conditions.

The results of this study suggest that if an extended winter shipping season resulted in a substantial loss of protective ice cover, one possible consequence would be a change in the nature of river load. It is possible that river load would increase as heavier sedimentary matter became more readily resuspended from the river floor. Furthermore, current velocities could increase under windy conditions. This potentially could affect the benthic and fish community, including overwintering eggs.

Benthos was generally a minor component of total river load. Unlike zooplankton, benthic drift (in terms of biomass) was not greater in the more rapidly flowing channel areas than in shallow regions. Benthic drift apparently was not higher in surface and mid-depth strata where current velocities were high when contrasted with bottom waters where current velocities were less. In many instances, there were significant day-night differences in benthos biomass (all winter comparisons; June Lake Nicolet and Point aux Frenes comparisons) suggesting that benthic organisms were able to retain their desired position in the water column over a 24-hour period. A possible exception was Frechette Point in June when current velocities may have been sufficiently high $(38.4 \mathrm{~cm} / \mathrm{s})$ to prevent the benthos from migrating down to the sediments during daylight hours. At all other locations and times, current velocities apparently were not sufficiently high to prevent benthic organisms from returning to the sediments following sunrise.

Plants were minor components of river load, especially in winter. The distribution of plant fragments within the river was fragments. In contrast, during the June study, zooplankton was a samples containing relatively large amounts of vegetation. Plants were most abundant at Frechette Point although the reason for this was not determined. Possibly there were greater standing stocks of plants upriver of Frechette Point. The effects of an extended winter shipping season on plants is unknown. Assuming that there was greater wave activity in the absence of ice cover, greater amounts of plant material could appear in the river load. This may or may not be beneficial to the river ecosystem. An increase in plant fragments potentially could benefit the benthic community (by supplying increased organic matter) although, if fragmentation was severe, the overwintering plant community could be harmed.

Fish larvae were minor components of river load. It is probable that $\mathrm{f}$ ish larvae biomass may have been underestimated by the sampling method used. Since $f$ ish larvae were not collected during winter, increased winter shipping should not have an effect on larval fish distributions at that time. However, as 
stated earlier, overwintering demersal eggs and pelagic juveniles and adults may be adversely affected by increased resuspension of detrital and mineral matter.

\section{FISH LARVAE}

\section{Lake Herring}

Historical and current evidence shows that lake herring spawn in the St. Marys River (Behmer et al. 1980; Goodyear et al. 1982; Liston et al. 1983, 1986). Newly hatched lake herring larvae that we collected established that spawning occurred in 1985. Certainly, suitable depths and bottom types are available in the river system. The scarcity of larvae in the Edison Hydropower Canal suggests that Lake Superior was not a major source.

Lake herring hatching usually begins from 1 to 6 days and peaks from 5 to 20 days after ice break-up (Colby and Brooke 1973; Cucin and Faber 1985). We estimate that ice break-up occurred around 20-22 April 1985 on the St. Marys River. Since we began sampling on 26 April, our collections should have covered the peak hatching period. Our results and those of Liston et al. (1983, 1986) generally show peak larval $f$ ish densities during the first 3 sampling wk after ice break-up. However, no distinct hatching peak, that is, a consistently maximum catch at all or most stations during only one sampling week, occurred. These findings suggest that either the hatching peak was missed or the sampling sites were not located near productive spawning sites. We believe the latter reason. However, the former cannot be dismissed totally. John and Hasler (1956) found that increased light and agitation stimulate the movement of lake herring embryos which accelerates larval emergence. Hatchery-reared eggs subjected to continuous illumination hatched 7 to 8 days earlier than eggs held in darkness. When kept in hatchery trays in a calm water bath, eggs that were ready to hatch were induced to hatch almost instantly by agitating the tray for a moment; when not disturbed, the eggs remained unhatched for several additional days (exact number not given) (John and Hasler 1956). Since ship traffic in the St. Marys River begins before the river is completely ice free, we can hypothesize that the agitation caused by ship passages and the increased illumination from the break-up of channel ice could cause early emergence of lake herring from eggs laid near the shipping channel. In addition, there is one reported incidence, over $2 \mathrm{yr}$, of lake herring emergence 4 to 5 wk before ice breakup in an Ontario lake (see Cucin and Faber 1985: page 24). 
Compared with peak densities of some spring-hatched species, the production of lake herring larvae in the St. Marys River appears to be moderate (Table 43). Densities of rainbow smelt and yellow perch were substantially greater than lake herring. However, densities of lake herring in the St. Marys River are similar to larval $f$ ish densities in productive areas of Lake Superior (Selgeby et al. 1978) and Lake Huron (Loftus 1979a, 1979b, 1982). Maximum densities from these three water bodies were in the same order of magnitude. Liston et al. (1986) concluded that lake herring is an abundant species which supports a major sport $f$ ishery in the St. Marys River. Goodyear et al. (1982) reported that lake herring spawn in the river, but no information was given on the magnitude of this reproduction.

Since lake herring are pelagic spawners, and the slightly adhesive eggs are deposited in shallow depths over no particular bottom type (Smith 1956; Colby and Brooke 1973; Scott and Crossman 1973; Cucin and Faber 1985), the newly hatched larvae tend to be widely distributed (Cucin and Faber 1985). Consequently there may be no major spawning area (that is, an area where intensive spawning occurs) in the St. Marys River.

Regarding larval $f$ ish production among the six river stations, East Neebish Island (station 6) appeared to be the most productive. Moderate spawning must have occurred in or upstream of this area. Some spawning apparently occurred at north Lake Nicolet and north Lake Munuscong (stations 2 and 7 ). Liston et al. (1986) collected moderate numbers of lake herring larvae at these two stations. Stations 2,6 , and 7 are all near small islands which may play a role in lake herring spawning or larval fish behavior. The remaining three stations in Lake $\mathrm{Nicolet}$ appear unimportant regarding lake herring reproduction. Larvae may have emigrated into these three stations after emerging elsewhere. Lake herring larvae are positively phototactic during the first few days after hatching and swim toward the surface. They then move to shallow inshore areas (Cucin and Faber 1985).

\section{Lake Whitefish}

Historically, lake whitefish spawned in the St. Marys River (Goodyear et al. 1982). Lake whitefish spawn in shallow depths, usually over sand, gravel, and rocks (Scott and Crossman 1973). Certainly this type of habitat is available in the st. Marys River, Although currently some spawning occurs in the river (Goodyear et al. 1982), the importance of this spawning appears to have diminished in recent years (Liston et al. 1986). Liston et al. (1986) did not consider lake whitefish to be an abundant species in the river. However, densities of larvae that we and liston et al. (1986) found in the river are similar in magnitude 
to densities reported from productive areas of Lake Huron (Loftus 1979a, 1979b, 1982).

The data on the number of lake whitefish and lake herring caught per week and the percentages of larvae that contained yolk showed two regular patterns that would be expected for larval fish that are residents of the St. Marys River system. First, the number collected declined in a regular fashion over the period of the study. We attributed this to natural mortality of larvae which is sometimes high during the first few days and weeks of their lives when it is critical that they obtain food. Second, as larval fish grow older they develop more fins and greater agility which helps them to avoid plankton nets towed in their environment. Since no lake whitefish and very few lake herring larvae passed through the Edison Hydropower Canal (station 1) during any given week's sampling, we concluded that the contribution from Izaak Walton Bay and Lake Superior to the St. Marys River population was very small. However, in 1983, Liston et al. (1986) found the greatest densities of lake whitefish larvae were at their Lake Superior station (near Izaak Walton Bay) compared with six other stations below the St. Marys Rapids. Densities in 1982 were much lower at this station than in 1983. Thus, in some years, lake whitefish production can be substantial in Lake Superior near the St. Marys River. We would expect lake herring and lake whitefish to hatch some days later in the Lake Superior area than in the St. Marys River, where water should heat faster and ice break up (cues for hatching) sooner than in Lake Superior. However, lake herring larvae caught in the Hydropower Canal entering the river from Lake Superior were low in abundance over the entire 6-wk period, and we collected no lake whitefish larvae in the canal.

Our data establish the St. Marys River as a spawning and nursery area for lake herring and lake whitefish. Larval $f$ ish densities of both species were similar in magnitude to densities reported from Lake Huron and Lake Superior. However, the importance of this reproduction to the river populations remains unanswered. One aspect of this question might be answered by sampling in an area away from the channelized portion of the river. The area around the north side of St. Joseph Island appears, at least from physical maps, to have suitable areas for coregonine spawning and, presumably, has not been disturbed by either dredging or large ship traffic. Densities of larval lake herring and lake whitefish in this relatively undisturbed area could be compared with those in the river where peak densities occurred in the past. 
Impacts of Winter Navigation on Fish

The impact on $f$ ish of the proposed extension of winter navigation past the normal closing date of 15 December must be examined in light of the life history of each of the important species that inhabits and reproduces in the area. Lake herring and lake whitefish spawn in the fall during November to early December; lake whitefish usually spawn over rocky, shallow areas while lake herring are pelagic spawners in shallow water with no particular preference for substrate type. One of the critical periods in the reproductive phase occurs just after fertilization and water hardening of the eggs. If eggs are subjected to increased agitation during this critical period, increased mortality can result. Since ship passages already occur over the spawning period, this potential impact has existed for many years. Another critical period for the St. Marys River coregonids is hatching in the spring. Lake herring eggs begin hatching from 1 to several days after ice break-up (Colby and Brooke 1973; Cucin and Faber 1985). There is also evidence that increased light and agitation can speed up larval emergence (John and Hasler 1956). If ice break-up in the St. Marys River occurs prematurely because of ship traffic in the spring, timing of lake herring emergence and seasonal production of its zooplankton and rotifer food may be mis-matched. Increased mortality of coregonid larvae could be the end result. So in the spring the potential for damage already exists as the navigation season usually opens on 1 April. Ice broken up early by ship traffic may cause early emergence of lake herring larvae and presumably lake whitefish. There is evidence (see Cucin and Faber 1985) of lake herring hatching under the ice on two occasions in an Ontario lake. However, there was only this one reported incident; all other studies show hatching just after ice break-up (John and Hasler 1956; Colby and Brooke 1973; Scott and Crossman 1973; Cucin and Faber 1985).

Another species which has the potential to be impacted by winter navigation is the burbot. The burbot spawns from December to April (Auer 1982), mostly during the winter and sometimes under ice in shallow water over sand or gravel shoals. Eggs are semibuoyant and scattered randomly over the substrate (Auer 1982). Our larval burbot data indicate that adults spawned over a prolonged period or that eggs were deposited in habitats varying greatly in water temperature. We collected larval burbot in moderate abundances and of the same newly hatched size in each of the 6 wh of the study. Since eggs of the burbot are semibuoyant, there is considerable potential for their movement from optimal spawning substrate because of the currents and waves generated by ship passage in the St. Marys River. Apparentiy this impact was not extremely detrimental as moderate numbers of burbot eggs hatched over the entire 6 wk of the study. 
Resuspension of sediments may be an important impact on overwintering fish eggs. The surge caused by ship passage in winter may resuspend fine sediments which could resettle on the eggs. Suffocation of the embryos would result in increased mortality for all three species.

If extension of the shipping season results in substantial ice breaking activity, another impact on overwintering eggs could occur. Channel ice pushed into shallow areas could scour the bottom. Overwintering coregonid and burbot eggs may be crushed, dislodged, or moved to unsuitable habitats.

Another possible effect on lake herring, lake whitefish, and burbot reproduction is the possible dislodgement of spawned eggs from the spawning substrate by the surge from ship passages. However if the $f$ ish are spawning on optimal substrates, some eggs should be deposited deep in the interstices of rocks, water harden and expand there, and be subject to very little dislodgement due to current and waves caused naturally or by ship passage. 


\section{LITERATURE CITED}

Auer, N.A. (ed.) 1982. Identification of larval fishes of the Great Lakes Basin with emphasis on the Lake Michigan drainage. Great Lakes Fish. Comm. Spec. Pub. 82-3. Ann Arbor, MI. 744 pp.

Bailey, P.C.E. 1981. Insect drift in Condor Creek, Australian capital territory. Aust. J. Mar. Freshwater Res. 32:111120.

Behmer, D.J., G.R. Gleason, and T. Gorenflo. 1980. Identification and evaluation of lake whitefish and herring spawning grounds in the St. Marys River area. Lake Superior State College, Sault Ste. Marie, MI. 29 pp.

Bhowmik, N.G., J.R. Adams, A.P. Bonini, C. Guo, D. Kisser, and M. Sexton. 1981. Resuspension and lateral movement of sediment by tow traffic on the Upper Mississippi and Illinois rivers. Rept. for the Env. Work Team, Upper Miss. Riv. Basin Comm. Master Plan, Minn., MN. III pp.

Bishop, J.E., and H.B.N. Hynes. 1969. Downstream drift of the invertebrates in a stream ecosystem. Arch. Hydrobiol. $66: 56-90$.

Colby, P.J., and L.T. Brooke. 1973. Effects of temperature on embryonic development of lake herring (Coregonus artedii). J. Fish. Res. Board Can. 30:799-810.

Cucin, D., and D.J. Faber. 1985. Early life studies of lake whitefish (Coregonus clupeaformis), cisco (Coregonus artedii) and yellow perch (Perca flavescens) in Lake Opeongo, ontario. Ontario Fish. Tech. Rep. Ser. No. 16. $28 \mathrm{pp}$.

Duffy, W., T. Batterson, and C. McNabb. 1987. The St. Marys River River, Michigan: An ecological profile. USFWS Great Lakes National Program Office, Biological Report 85(7.10) of National Wetlands Research Center, Washington, D.C. 20240.

Eckblad, J.W. 1981. Baseline studies and impacts on navigation on the benthos and drift, on the quantity of flow to side channels and on the suspended matter entering side channels of Pool 9 of the Upper Mississippi River. Rept. for the Env. Work team, Upper Miss. Riv. Basin Comm. Master Plan, Minn, MN. 148 pp.

Eckblad, J.W., C.S. Volden, and L.S. Weilgart. 1983. hllochthonous drift from backwaters to the main channel of the Mississippi River. Amer. Midl. Nat. 111:16-22. 
Elliott, J.M. 1967. Invertebrate drift in a Dartmoor stream. Arch. Hydrobiol. 63:202-237.

Elliott, J.M. 1970. Methods of sampling invertebrate drift in running water. Ann. Limnol. 6:133-159.

Elliott, J.M. 1971. The distances travelled by drifting invertebrates in a Lake District stream. Oecologia 6:350379 .

Evans, M.S., and D.W. Sell. 1985. Mesh size and collection characteristics of $50-\mathrm{cm}$ diameter conical plankton nets. Hydrobiologia 122:97-194.

Fassett, N.C. 1957. A manual of aquatic plants. Univ. Wis. Press, Madison, Wis.

Ghetti, P.F., and U. Ravanetti. 1984. The drift over one year of Plecoptera and Ephemeroptera in a small stream in northern Italy. Arch. Hydrobiol. 99:478-488.

Goodyear, C.D., T.A. Edsall, D.M. Ormsby Dempsey, G.D. Moss, and P.E. Polanski. 1982. Atlas of the spawning and nursery areas of Great Lakes fishes. Vol. 3: St. Marys River. U.S. Fish and Wildl. Serv., Washington, D.C. FWS/OBS-82/52.

Herricks, E.E. 1981. Effects of barge passage on physical, chemical and biological conditions in the navigation reach of the Kaskaskia River. Rept. for the Env. Work Team, Upper Miss. Riv. Basin Comm. Master Plan, Minn., MN. 98 pp.

Hilsenhoff, W.L. 1975. Aquatic insects of Wisconsin. Tech. Bull. No. 89. Dept. of Nat. Res., Madison, Wisc. 53 pp.

John, K.R., and A.D. Hasler. 1956. Observations on some factors affecting the hatching of eggs and the survival of young shallow-water cisco, Leucichthys artedi LeSueur, in Lake Mendota, Wisconsin. Limnol. Oceanogr. 1:176-194.

Lind, O.T. 1979. Handbook of common methods in limnology. Second edition. C.V. Mosby Co., St. Louis, MO.

Liston, C.R. and C. D. MCNabb. 1986. Environmental baseline studies during 1984 of Lake Nicolet and Munuscong Bay, St. Marys River, Michigan, in relation to proposed extension of the navigation season. Office of Biol. Services, Fish and Wildlife Service, U.S. Dept. of Interior, Twin Cities, Minnesota 55111, Biological Report $86(3), 99$ pp. plus appendices. 
Liston, C.R., C. McNabb, W. Duffy, D. Ashton, R. Ligman, F. Koehler, J. Bohr, G. Fleischer, J. Schuette, and R. Yanusz. 1983. Environmental baseline studies of the St. Marys River near Neebish Island, Michigan, prior to proposed extension of the navigation season. U.S. Fish and Wildl. Serv., Washington, D.C. FWS/OBS-80/62.2. 316 pp.

Liston, C.R.., C. D. McNabb, D. Brazo, J. Bohr, J. Craig,

W. Duffy, G. Fleischer, G. Knoecklein, F. Koehler, R. Ligman, R. O'Neal, M. Siami, and P. Roettger. 1986. Limnological and $f$ isheries studies of the St. Marys River, Michigan, in relation to proposed extension of the navigation season, 1982 and 1983. Office of Biol. Services, Fish and Wildlife Service, U.S. Dept. of Interior, Twin Cities, Minnesota 551ll. Biological Report 85(2), 764 pp. plus vol. 2, Appendices.

Loftus, D.H. 1979a. North Channel larval fish survey: Joe Dollar Bay to Aird Bay, May, 1979. Rep. 2-79. Lake Huron Fish. Assessment Unit, Ont. Min. Nat. Res., Owen Sound, Ont. $33 \mathrm{pp}$.

Loftus, D.H. 1979b. Larval fish sampling in Lake Huron, 1979. Rep. 3-79. Lake Huron Fish. Assessment Unit., Ont. Min. Nat. Res., Owen Sound, Ont. 21 pp.

Loftus, D.H. 1982. Larval fish sampling in Lake Huron, 1978. Ont. Fish. Tech. Rep. Ser. No. 2. Ont. Min. Nat. Res., Fish. Branch, Toronto, Ont. $12 \mathrm{pp}$.

Logan, S.M. 1963. Winter observations on bottom organisms and trout in Bridger Creek, Montana. Trans. Amer. Fish. Soc. 92:140-145.

Mansfield, P.J., D.J. Jude, D.T. Michaud, D.C. Brazo, and J. Gulvas. 1983. Distribution and abundance of larval burbot and deepwater sculpin in Lake Michigan. Trans. Amer. Fish. Soc. 112:162-172.

Matter, W.J., and A.H. Hopwood. 1980. Vertical distribution of invertebrate drift in a large river. Limnol. Oceanogr. 25:1117-1121.

McLay, C.L. 1970. A theory concerning the distance travelled by animals entering the drift of a stream. J. Fish. Res. Board Can. $27: 359-370$.

Mendenhall, W. 1971. Introduction to probability and statistics. Third edition. Duxbury Press, Belmont, Calif. 
Morgan, C.C., and A.D. Waddell. 1981. Diurnal variation in the emergence of some aquatic insects. Trans. Royal

Entomol. Soc. London 113:123-137.

Morris, L.A., R.N. Langemeier, T.R. Russell, and A. Witt, Jr. 1968. Effects of main stem impoundments and channelization upon the limnology of the Missouri River, Nebraska. Trans. Amer. Fish. Soc. 97:380-388.

Muller, K. 1954a. Investigations on the organic drift in north Swedish streams. Rep. Inst. Freshwat. Res. Drottningholm $35: 133-148$.

Muller, K. 1954b. Die Drift in fliessenden Gewassern. Arch. Hydrobiol. 49:539-545.

Mundie, J.H. 1956. The biology of flies associated with water supply. J. Inst. Publ. Hith. Engrs. 55:178-193.

Pearson, W.D. 1970. Drift of Oligophlebodes sigma and Baetis bicaudatus in a mountain stream. Ph.D. thesis. Utah State Univ., Logan, UT.

Pearson, W.D., and D.R. Franklin. 1968. Some factors affecting drift rates of Baetis and Simuliidae in a large river. Ecology $49: 75-81$.

Poe, T.P., and T.A. Edsall. 1982. Effects of vessel-induced waves on the composition and amount of drift in an ice environment in the St. Marys River. Admin. Rep. 82-6, U.S. Fish and Wildl. Serv., Ann Arbor, MI.

Poe, T.P., T.A. Edsall, and J.K. Hiltunen. 1980. Effects of vessel-induced waves in an ice environment on the St. Marys River. Admin. Rep. 80-6. U.S. Fish and Wildlife

Serv. Great Lakes Fish. Lab., Ann Arbor, MI. 125 pp.

Prescott, G.W. 1962. Algae of the western Great Lakes area. Wm. C. Brown Co., Dubuque, Iowa.

Scott, W.B., and E.J. Crossman. 1973. Freshwater fishes of Canada. Fish. Res. Board Can. Bull. 184. 966 pp.

Seagle, H.H., and F.H. Zumwalt. 1981. Evaluation of the effects of tow passage on aquatic macroinvertebrate drift in Pool 26, Mississippi River. Rept. for the Env. Work Team, Upper Miss. Riv. Basin Comm. Master Plan, Minn., MN. 91 pp. 
Seagle, H.H., J.C. Hutton, and K.S. Lubinski. 1982. A comparison of benthic invertebrate community composition in the Mississippi and Illinois Rivers, Pool 26. J. Freshwat. Ecol. 1:637-650.

Seibel, E., C.T. Carlson, and J.W. Maresca, Jr. 1975. Lake and shore ice conditions on southeastern Lake Michigan in the vicinity of the Donald C. Cook nuclear plant: Winter 197374. Spec. Rep. No. 55. Great Lakes Research Div., Univ. of Mich., Ann Arbor, Mich. 62 pp.

Selgeby, J.H. 1975. Life histories and abundance of crustacean zooplankton in the outlet of Lake Superior, 1971-1972.

Can. J. Fish. Res. Board Can. 32:461-470.

Selgeby, J.H., W.R. MacCallum, and D.V. Swedberg. 1978. Predation by rainbow smelt (Osmerus mordax) on lake herring (Coregonus artedii) in western Lake Superior. J. Fish. Res. Board Can. 35:1457-1463.

Smith, S.H. 1956. Life history of lake herring of Green Bay, Lake Michigan. U.S. Fish Wildl. Serv. Fish. Bull. 109:87138.

Sokal, R.R., and F.J. Rohlf. 1969. Biometry. The principles and practice of statistics in biological research. W.H. Freeman and Co., San Francisco, CA. 776 pp.

United States Army Corps of Engineers. 1984. St. Marys River oil/toxic substance spill study. Current velocities and directions, 1980-1983. U.S. Army Corps of Engineers, Detroit District, Great Lakes Hydraulics and Hydrology Branch. 13 pp. + Appendices.

Voss, E.G. 1972. Michigan flora. Part I, Gymnosperms and monocots. Cranbrook Inst. Sci. and Univ. Mich. Herbarium. Bulletin 55 .

Waters, T.F. 1961. Standing crop and drift of stream bottom organisms. Ecology 42:532-537.

Waters, T.F. 1962a. A method to estimate production rate of a stream bottom invertebrate. Trans. Amer. Fish. Soc. 91:243250.

Waters, T.F. 1962b. Diurnal periodicity in the drift of stream invertebrates. Ecology 43:316-320.

Waters, T.F. 1966. Production rate, population density, and drift of a stream invertebrate. Ecology 47:595-604. 
Waters, T.F. 1972. The drift of stream insects. Ann. Rev. Entomol. $17: 253-272$.

Waters, T.F. 1981. Seasonal patterns in production and drift of Gammarus pseudolimnaeus in Valley Creek, Minnesota. Ecology $62: 1458-1466$. 
